\title{
AN OPTIMAL CONTROL STRATEGY TO DETERMINE DIFFUSIVITY VERSUS CONCENTRATION IN TERNARY SYSTEMS OF TWO GASES AND A NON VOLATILE PHASE
}

By

Amir Jalal Sani

M.Sc in Applied Chemistry, University of Karachi, Karachi, 1993

M.A.Sc in Chemical Engineering, Ryerson University, Toronto, 2010

A dissertation presented to

Ryerson University

In the partial fulfillment of the

Requirements for the degree of

Doctor of Philosophy

In the program of

Chemical Engineering

Toronto, Ontario, Canada, 2014

(C) Amir Jalal Sani 2014 


\section{Author's Declaration}

I here declare that I am the sole author of this dissertation. This is a true copy of the dissertation, including any required final revisions, as accepted by my examiners.

I authorize Ryerson University to lend this dissertation to other institutions or individuals for the purpose of scholarly research.

I further authorize Ryerson University to reproduce this dissertation by photocopying or by other means, in total or in part, at the request of other institutions or individuals for the purpose of scholarly research. 


\title{
AN OPTIMAL CONTROL FRAMEWORK TO DETERMINE DIFFUSIVITY VERSUS CONCENTRATION SURFACES IN TERNARY SYSTEMS OF TWO GASES AND A NON VOLATILE PHASE Doctor of Philosophy 2014 Amir Jalal Sani Chemical Engineering Ryerson University
}

\begin{abstract}
Diffusivity is a strong function of concentration and an important transport property. Diffusion of multiple species is far more frequent than the diffusion of one species. However, there are limited experimental data available on multi-component diffusivity. The objective of this study is to develop an optimal control framework to determine multicomponent, concentration-dependent diffusivities of two gases in a non-volatile phase such as polymer.

In Part 1 of this study, we derived a detailed mass-transfer model of the experimental diffusion process for the non-volatile phase to provide the temporal masses of gases in the polymer. The determination of diffusivities is an inverse problem involving principles of optimal control. Necessary conditions are determined to solve this problem.

In Part 2 of this study, we utilized the results of Part 1 to determine the concentrationdependent, multi-component diffusivities of nitrogen and carbon dioxide in polystyrene. To
\end{abstract}


that end, solubility and diffusion experiments are conducted to obtain necessary data. In the ternary system of nitrogen (1), carbon dioxide (2), and polystyrene (3), the diffusivities $D_{11}, D_{12}, D_{21}$, and $D_{22}$ versus the gas mass fractions are two-dimensional surfaces.

The diffusivity of carbon dioxide was found to be greater than that of nitrogen. The value of the main diffusion coefficient $D_{11}$ was found to increase as the concentration of carbon dioxide increased. The highest value of $D_{11}$ obtained was $2.22 \times 10^{-8} \mathrm{~m}^{2} \mathrm{~s}^{-1}$ for nitrogen mass fraction of $3.14 \times 10^{-4}$ and for a carbon dioxide mass fraction of $5.67 \times 10^{-4}$. The cross-diffusion coefficient $D_{12}$ increased as the concentrations of nitrogen and carbon dioxide increased. The diffusivity reached its maximum value when the concentrations of nitrogen and carbon dioxide were at their maximum values. The diffusivity was of the order of $10^{-9} \mathrm{~m}^{2} \mathrm{~s}^{-1}$

The diffusivity of the cross-diffusion coefficient $D_{21}$ was found to be increased for the mass fractions of carbon dioxide ranging from 0 to $1.70 \times 10^{-3}$. The diffusivity was found to be of the order of $10^{-7} \mathrm{~m}^{2} \mathrm{~s}^{-1}$. The diffusion coefficient, $D_{22}$, was found to increase with the concentrations of nitrogen and carbon dioxide, $D_{22}$ remained high with low concentrations of carbon dioxide. The diffusivity was found to be of the order of $10^{-7} \mathrm{~m}^{2} \mathrm{~s}^{-1}$. 


\section{Acknowledgement}

I take this opportunity to express my profound gratitude and deep regards to my guide

Professor Dr. Simant R. Upreti for his exemplary guidance, monitoring and constant encouragement throughout the course of this thesis. The blessing, help and guidance given by him time to time shall carry me a long way in the journey of life on which I am about to embark.

I also take this opportunity to express a deep sense of gratitude to Professor Dr. Farhad Ein-Mozaffari and Professor Dr. Jiangning Wu for their cordial support, valuable information and guidance, which helped me in completing this task through various stages.

I am obliged to engineering specialists Ali Hemmati, Daniel Boothe, Tondar Tajrobehkar, and technical specialist Shawn McFadden, for the valuable information provided by them in their respective fields. I am grateful for their cooperation during the period of my assignment.

My completion of this project could not have been accomplished without the support of my children, thank you for allowing me time away from you to research and write. To my caring, loving, and supportive wife, Yasmin: my deepest gratitude. Your encouragement when the times got rough are much appreciated and duly noted.

Lastly, I thank almighty without which this assignment would not be possible.

Amir Sani 


\section{Dedication}

I dedicate this work to my spiritual mentors

Khwaja Shamsuddin Azeemi

and

Master Choa Kok Sui 


\section{Table of Content}

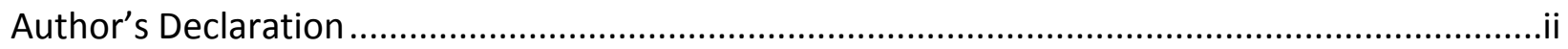

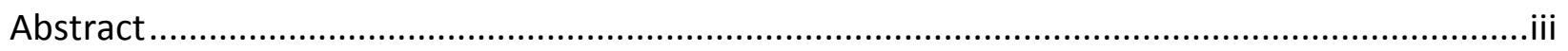

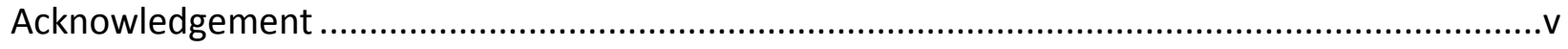

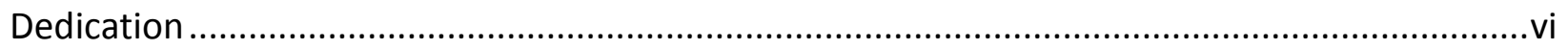

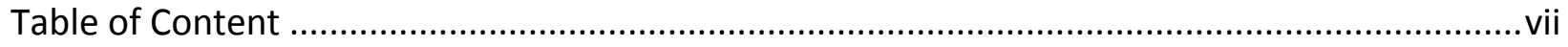

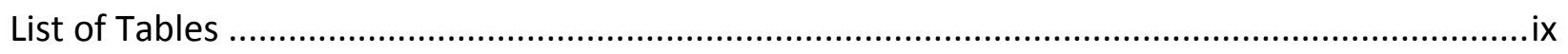

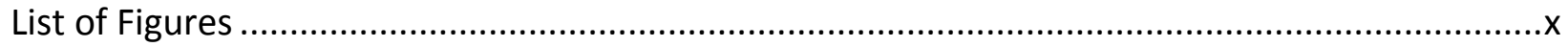

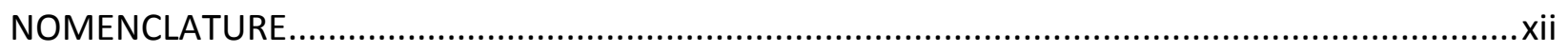

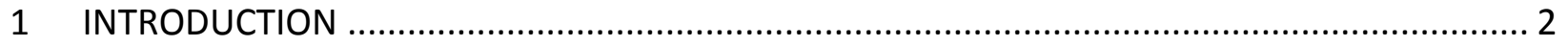

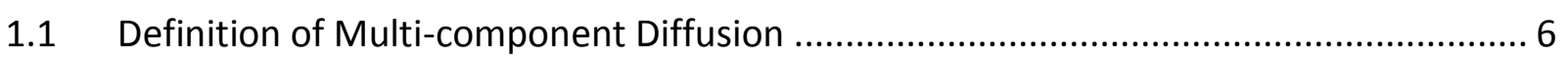

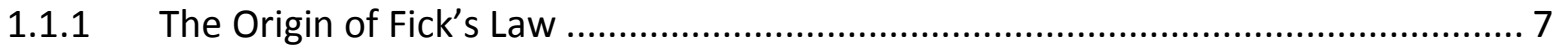

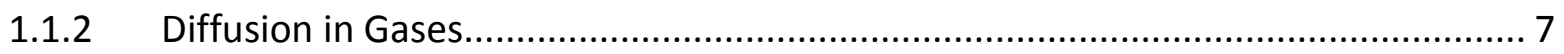

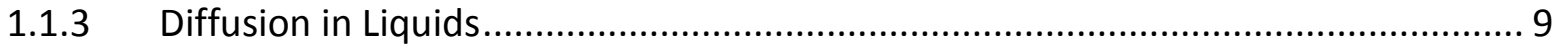

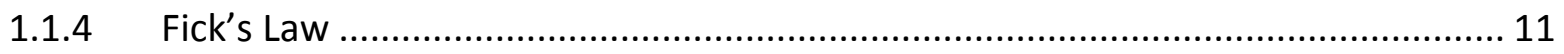

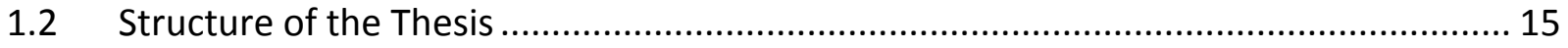

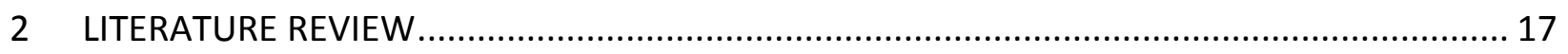

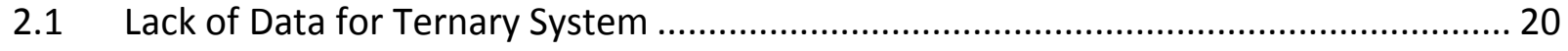

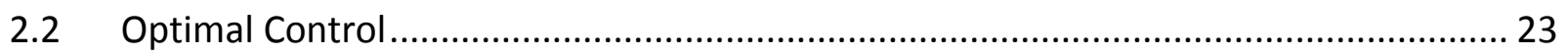

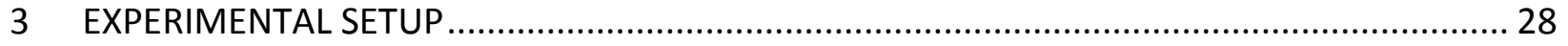

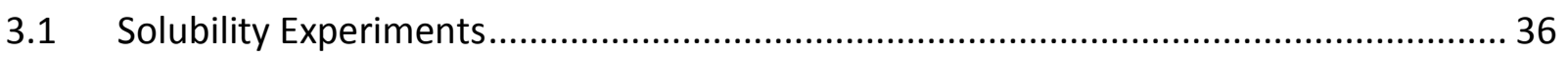

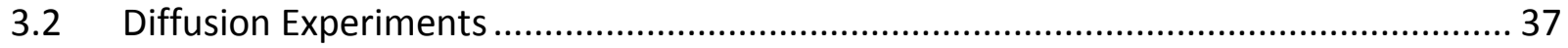

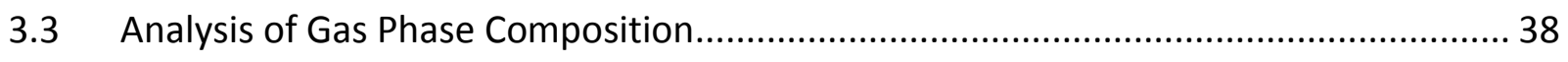

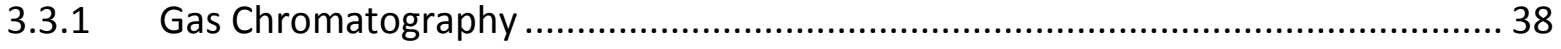

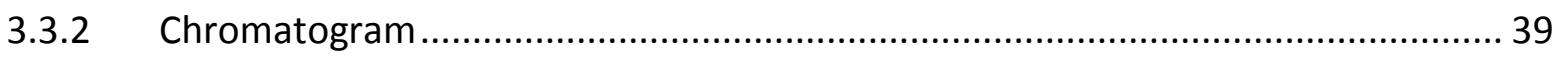

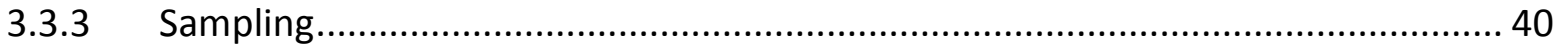

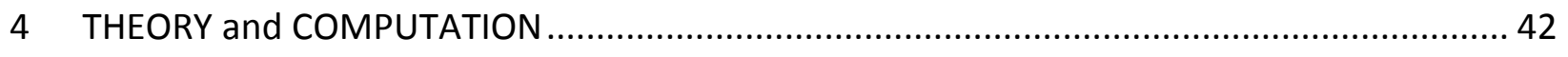

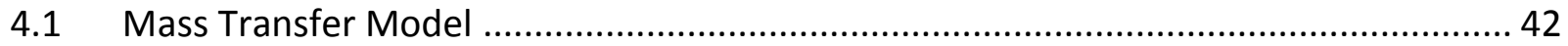




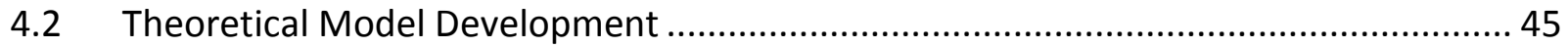

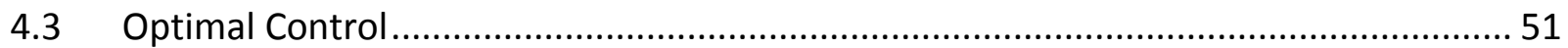

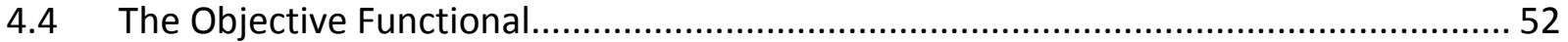

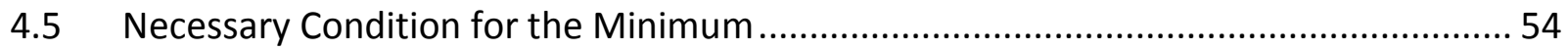

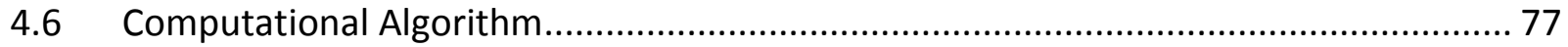

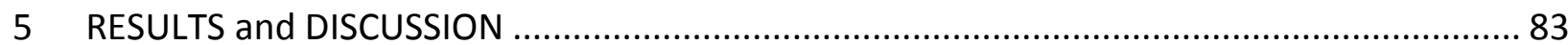

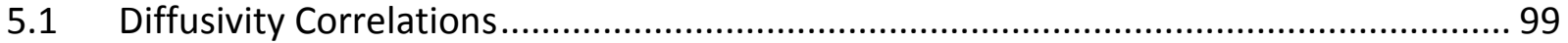

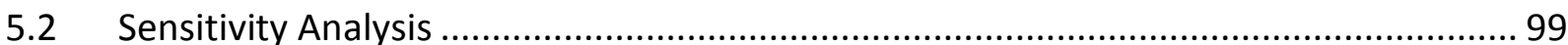

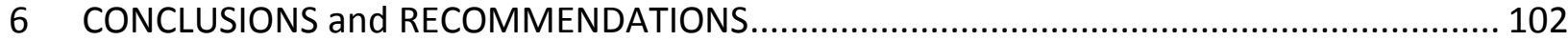

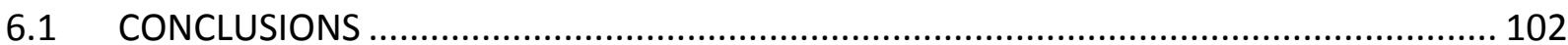

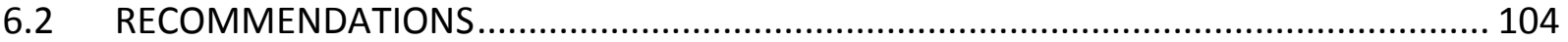

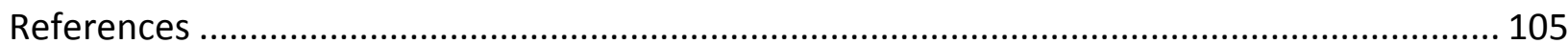

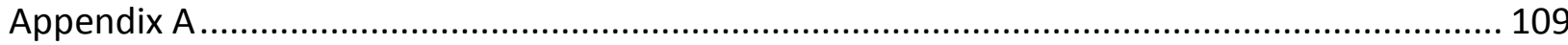

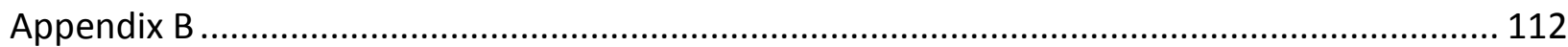

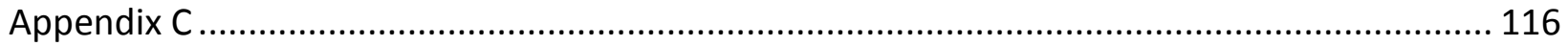

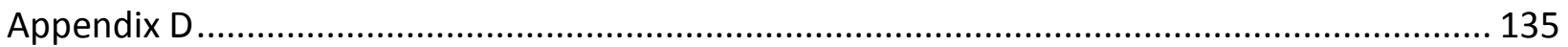




\section{List of Tables}

Table 2.1 Diffusion coefficients of carbon dioxide and nitrogen in polystyrene......

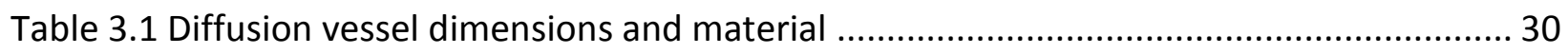

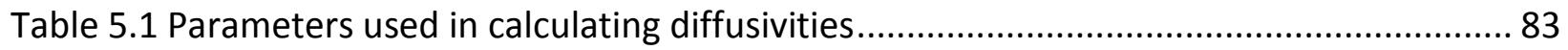

Table 5.2 Various trials for obtaining optimal diffusivities.................................................. 84

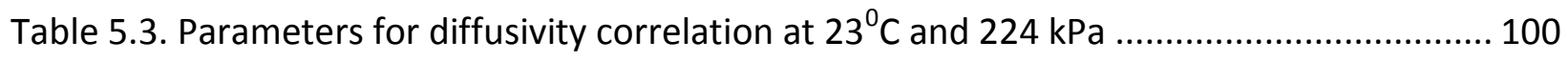

Table 5.4. Sensitivity analysis $\pm 3 \%$ change in the estimate ............................................ 101 


\section{List of Figures}

Figure 1.1. Two homogeneous mixtures separated by a polymer membrane 3

Figure 1.2. Equimolar mixture of hydrogen and argon in Tube 1; equimolar mixture of methane and argon in Tube 2 . 5

Figure 1.3. Nickel ions diffusing from an acidic solution through a membrane into a strong acid 6

Figure 1.4. Graham's apparatus for the study of diffusion 8

Figure 1.5. (a) Diffusion in bottles with salt solution; (b) empty bottle in a jar that contains only

water 10

Figure 1.6. Multi-component diffusion in nitrogen, carbon dioxide, and polystyrene 13

Figure 3.1. Experimental setup. 29

Figure 3.2 Polymer temperature during diffusion experiment 31

Figure 3.3 Polymer thickness during diffusion experiments .............................................. 32

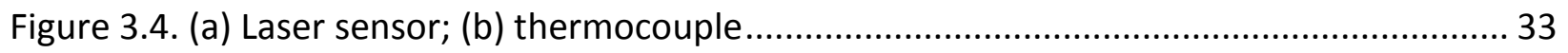

Figure 3.5. Polystyrene layer with uniform thickness ...................................................... 35

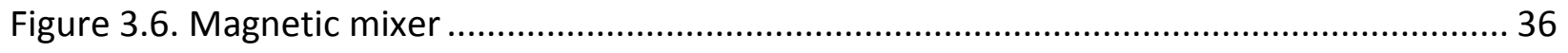

Figure 4.1. Diffusion of two gases in an underlying dense, non-volatile phase ....................... 44

Figure 4.2. Methodology for determining diffusivities ................................................. 80

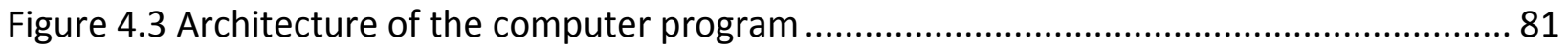

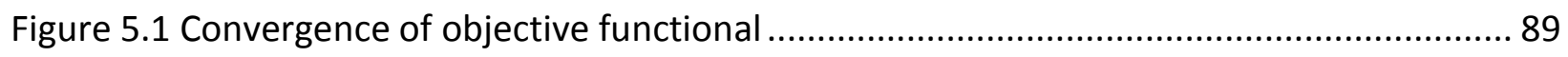

Figure 5.2 Experimental and calculated mass of carbon dioxide absorbed in polystyrene........ 90

Figure 5.3. Experimental and calculated mass of nitrogen absorbed in polystyrene ................ 90 
Figure 5.4. Interfacial mass fraction of nitrogen

Figure 5.5. Interfacial mass fraction of carbon dioxide .................................................. 92

Figure 5.6. Main diffusion coefficient, $D_{11}$, of nitrogen in polystyrene .................................. 93

Figure 5.7. Cross-diffusion coefficient, $D_{12}$, of nitrogen in polystyrene .................................. 94

Figure 5.8. Cross-diffusion coefficient, $D_{21}$, of carbon dioxide in polystyrene ......................... 95

Figure 5.9. Main diffusion coefficient, $D_{22}$, of carbon dioxide in polystyrene........................... 96

Figure 5.10. Ratio of cross diffusion coefficient $\left(D_{21} / D_{11}\right)$ of carbon dioxide due to the

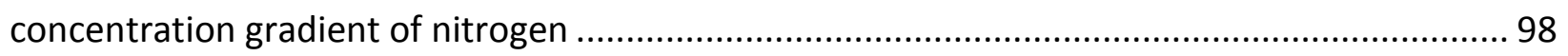

Figure 5.11. Ratio of cross diffusion coefficient $\left(D_{12} / D_{22}\right)$ of nitrogen due to the concentration gradient of carbon dioxide 98 


\section{NOMENCLATURE}

$D_{i, j} \quad$ where $\mathrm{i}, \mathrm{j}=1,2$. Diffusivity of gas or liquid component in a system, $\mathrm{m}^{2} \mathrm{~s}^{-1}$

G State equation, $\mathrm{kg} \mathrm{s}^{-1}$

I Objective functional

$J_{D} \quad$ Rate of mass transfer per unit area, $\mathrm{kg} \mathrm{m}^{2} \mathrm{~s}^{-1}$

J Augmented objective functional

$J_{1} \quad$ Rate of mass transfer per unit area in one direction, $\mathrm{kg} \mathrm{m}^{2} \mathrm{~s}^{-1}$

$L \quad$ Thickness of the polymer sample, $\mathrm{m}$

$N_{1} \quad$ Mass flux of $\mathrm{CO}_{2}, \mathrm{~kg}$ mole $\mathrm{m}^{2} \mathrm{~s}^{-1}$

$N_{2} \quad$ Mass flux of $\mathrm{N}_{2}, \mathrm{~kg}$ mole $\mathrm{m}^{2} \mathrm{~s}^{-1}$

$P \quad$ Pressure, $\mathrm{kPa}$

Temperature, ${ }^{0} \mathrm{C}$

$t \quad$ Total run time of the experiment, $\mathrm{s}$

$y_{i, j} \quad$ Where $\mathrm{i}, \mathrm{j}=1,2$ Gas-phase composition, $\mathrm{kg} \mathrm{m}^{-3}$

$z \quad$ Depth in the polymer, $\mathrm{m}$

\section{Greek Symbols}
$\rho \quad$ Density of polymer, $\mathrm{kg} \mathrm{m}^{-3}$
$\lambda \quad$ Costate variable defined by Equation
$\omega_{1} \quad$ Mass fraction of $\mathrm{N}_{2}$ 
$\omega_{\text {sat }} \quad$ Equilibrium concentration of gases at the surface of the polymer, $\mathrm{kg} \mathrm{m}^{-3}$

$\omega_{i, \bmod } \quad$ Model predicted value of mass of gas absorbed, $\mathrm{kg}$

$\omega_{i, \exp } \quad$ Experimental value of mass of gas absorbed, $\mathrm{kg}$ 


\section{INTRODUCTION}

Diffusion can be defined in very simple terms as the movement of molecules from one place to another due to a concentration gradient (Philibert, 2005). Chemical engineers frequently deal with situations in which three or more components move from one place to another at the same time. The conventional approach of mass transfer in a system is based on the assumption that the movement of a chemical species from one place to another is directly proportional to a driving force. The problem is that this assumption is only good for cases in which diffusion is occurring in a two-component system (binary system), in a system in which one component is diluted by a large excess of one or more of the other components, or in a system in which all of the components in the mixture have similar quantities and natures.

The question arises concerning whether the three or more components diffusing in a system can be dealt in a same way as the components of a binary system. Answer to this question is "No," so we must deal with the problem of how to deal with a multi-component system. Concerns such as this have been on the minds of chemical engineers for a long time(Krishnamurthy \& Taylor, 1982).

Multi-component diffusion systems exhibit characteristics that are quite different from those of binary systems. In addition, methods have been developed to predict multicomponent diffusion in a consistent way using matrix formulations (E. L. Cussler, 2009). The 
matrix of a multi-component system can be incorporated into powerful computer software, which can be used in equipment design. This is one practical application of the multi-component diffusion matrix.

Multi-component diffusion occurs when the flux of one component is influenced by the concentration gradient of a second component. For example, the flux of the first component can be increased by as much as an order of magnitude by changing the concentration gradient of the second component. In multicomponent diffusion, the first component can diffuse against its concentration gradient, i.e., from a region of lower concentration to the region of higher concentration. The following examples explain these effects in detail.

Figure 1.1 shows a tank that contains two homogeneous mixtures that are separated by a polymer membrane.

\begin{tabular}{|c|c|}
\hline$\underset{(1)}{\mathrm{Na}_{2} \mathrm{SO}_{4}}+\underset{2}{\mathrm{H}_{2} \mathrm{O}}$ & $\begin{array}{c}\mathrm{CH}_{3} \mathrm{COCH}_{3} \\
\text { (3) } \\
\mathrm{Na}_{2} \mathrm{SO}_{4}+\mathrm{H}_{2} \mathrm{O} \\
\text { (1) }\end{array}$ \\
\hline
\end{tabular}

Figure 1.1. Two homogeneous mixtures separated by a polymer membrane

The right side of the tank contains a solution of sodium sulfate, and the left side of the tank contains pure water. As the experiment begins, sodium sulfate diffuses from the right side of the tank to the left side of the tank, and the process continues until the concentrations 
of sodium sulfate are equal on both sides of the tank. At this point, if acetone is added to the right side of the tank, the diffusion rate of sodium sulfate to the left side of the tank increases. As more acetone is added, more sodium sulfate diffuses to the other side of the membrane. However, if acetone is added to the left side of the tank, the rate of diffusion of sodium sulfate is decreased, and, if acetone is added on both sides of the porous membrane, the diffusion of sodium sulfate is increased slightly. This shows that the gradient of acetone strongly influences the diffusion of sodium sulfate (E. L. Cussler \& Breuer, 1972).

For the second example, consider Figure 1.2 in which tube 1 contains an equimolar mixture of hydrogen and argon, and the bottom tube 2 contains an equimolar mixture of methane and argon. The two tubes were connected to start the experiment, and they were disconnected to end the experiment. Since the initial difference in the argon concentration in the two tubes was zero, it was predicted that the difference in the concentration of argon in the two tubes would remain zero. However, when the concentration of argon was measured in both tubes at the end of the experiment, it was found that the gradients of hydrogen and methane had influenced the gradient of argon (Arnold \& Toor, 1967).

Figure 1.3 presents a third example. A small drop of a strong acid was coated with a thin layer of liquid ion exchange resin and then immersed in a weak acidic solution of nickel. 


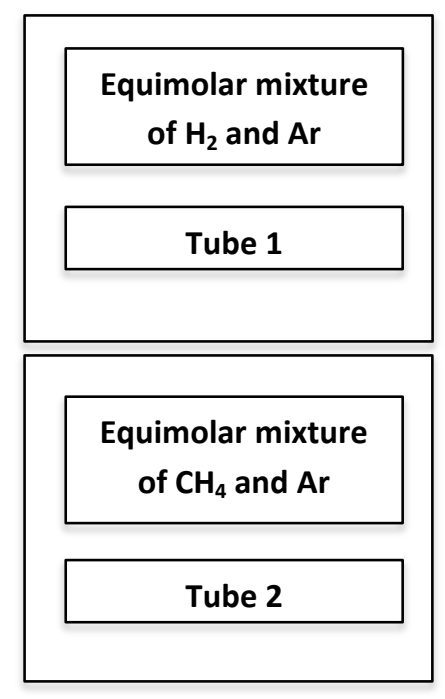

Figure 1.2. Equimolar mixture of hydrogen and argon in Tube 1; equimolar mixture of methane and argon in Tube 2.

At the beginning of the experiment, the nickel diffused from the outside of the drop into the drop across a membrane. But the diffusion of the nickel did not stop even when the concentration of nickel was the same on both sides of the membrane. Nickel continued to diffuse into the drop even when the concentration of nickel inside the drop was many times greater than the concentration outside the drop. In this example, the concentration difference of the acid causes a flux of nickel against its gradient. Above three examples shows multi-component diffusion in which the flux of one solute is a function of the gradient of the second solute. 


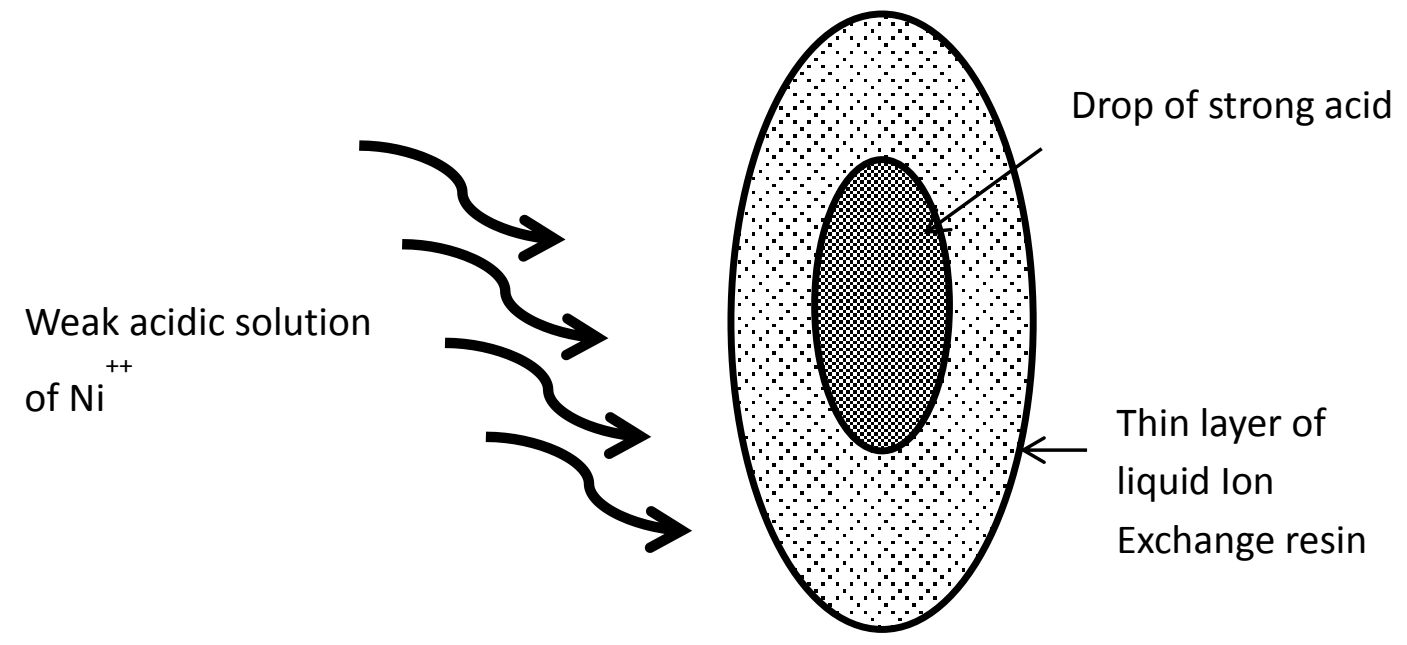

Figure 1.3. Nickel ions diffusing from an acidic solution through a membrane into a strong acid

\subsection{Definition of Multi-component Diffusion}

To understand multi-component diffusion, first, we need to discuss binary diffusion briefly because it forms the basis for the multi-component diffusion that is discussed in the rest of this thesis. We begin with Fick's law, which describes the basic relationships in binary diffusion. The diffusion coefficient described by this law is discussed for gases and liquids. Diffusion in solids is not covered because it is not relevant, so, after discussing gases and liquids, the structure of the thesis is presented. Then, multi-component diffusion is addressed, including the optimal control framework for determining multi-component diffusion 


\subsubsection{The Origin of Fick's Law}

Early studies of diffusion were split into studies of gases and liquids. Researchers interested in understanding the behaviors of atoms or molecules were focussed on studying gases. Researchers working in the areas of medicine and physiology wanted to understand biological transport processes, so they focused on the study of liquids, primarily the diffusion of liquids across membranes (Fick, 1995). Let's look briefly at the diffusion of gases and liquids.

\subsubsection{Diffusion in Gases}

Thomas Graham $(1829,1833)$ was the first to analyze the diffusion process quantitatively. Most of his research was conducted using the diffusion apparatus shown below in Figure 1.4. As shown in Figure 1.4, Graham's apparatus consisted of a glass tube, one end of which was immersed in water, with the other end closed by a stucco plug. The tube was filled with hydrogen. Initially, the hydrogen diffuses out of the tube through the stucco plug, while air diffuses into the tube from the outside.

The flux of hydrogen leaving the tube was not equal to the flux of air entering the tube, so the level of the water in the tube rises during diffusion (Graham, 1833). Graham observed that the rise in the water level in the tube would cause a pressure gradient that would 
adversely affect the diffusion process. So, Graham lowered the tube in the water so that the water level in the tube would remain constant. So, his experiments had the characteristic of changing the volume of each gas that was originally in the tube. Graham later showed that this change in volume was inversely proportional to the square root of the density of the gas.

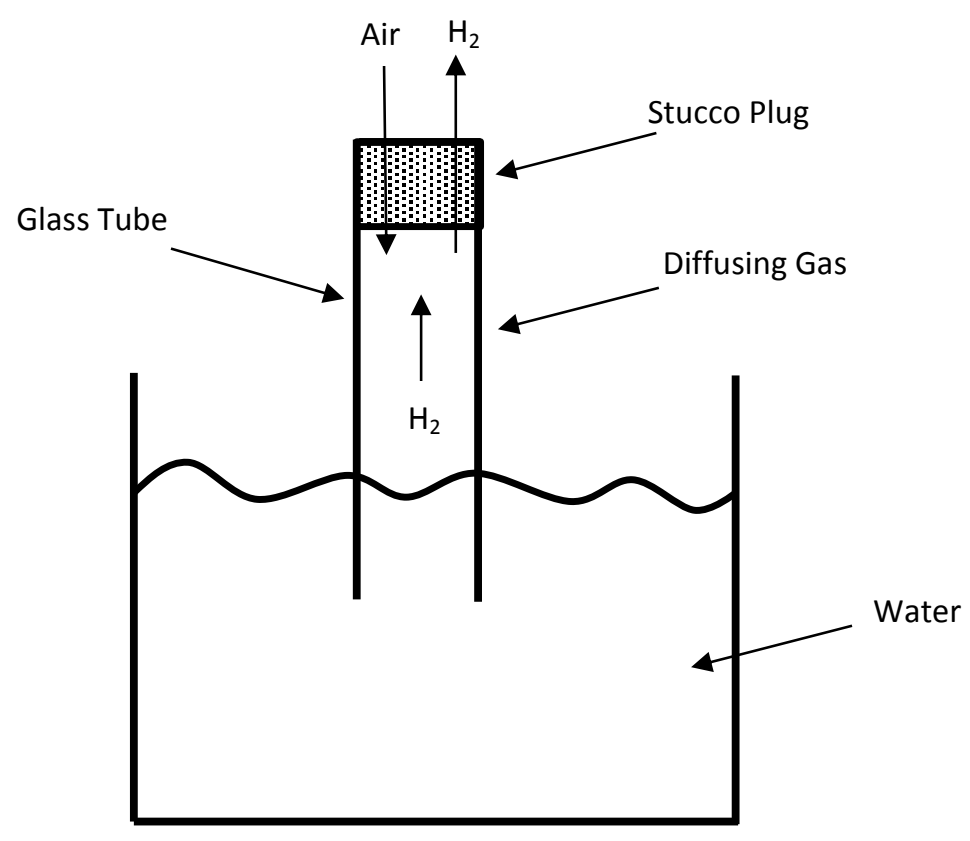

Figure 1.4. Graham's apparatus for the study of diffusion

Since the change in the volume of the gas in the tube was related to diffusion, Graham stated:

"Diffusion or spontaneous intermixture of two gases in contact is effected by an interchange of position on infinitely minute volumes, being, in the case of each gas, 
inversely proportional to the square root of the density of the gas..." (Graham, 1833). In other words, 'Diffusion is inversely proportional to the square root of the molecular weight of the gas".

Graham's diffusion experiments did not say anything about the diffusion coefficient of the gas. Since there was no pressure difference across the porous plug, the process of diffusion across the porous plug could be easily explained without any need of Fick's law or diffusion coefficients (Masom \& and Kronstadt, 1967). This is an example of an isobaric diffusion process rather than the equimolar diffusion process that commonly is used to measure diffusion coefficients. Graham's experiments attracted attention towards diffusion as an interesting molecular process, but he was unable to develop a basic diffusion law.

\subsubsection{Diffusion in Liquids}

The results of early experiments involving diffusion in liquids were difficult to interpret due to the presence of the membrane in the diffusion process. Fick quoted Von Bruke (1843), a physiologist who used olive oil and turpentine on the opposite sides of the leather membrane and then he measured the change in the volume due to diffusion. This experiment supported the hypothesis of the osmotic effect. But, the presence of the membrane made the analysis of the diffusion process difficult. 
In 1850 (Graham, 1850), Graham did a remarkable job of clarifying the diffusion process in liquids. To understand the diffusion process in the presence of a membrane, he conducted a series of experiments using the setup shown in Figures 1.5.

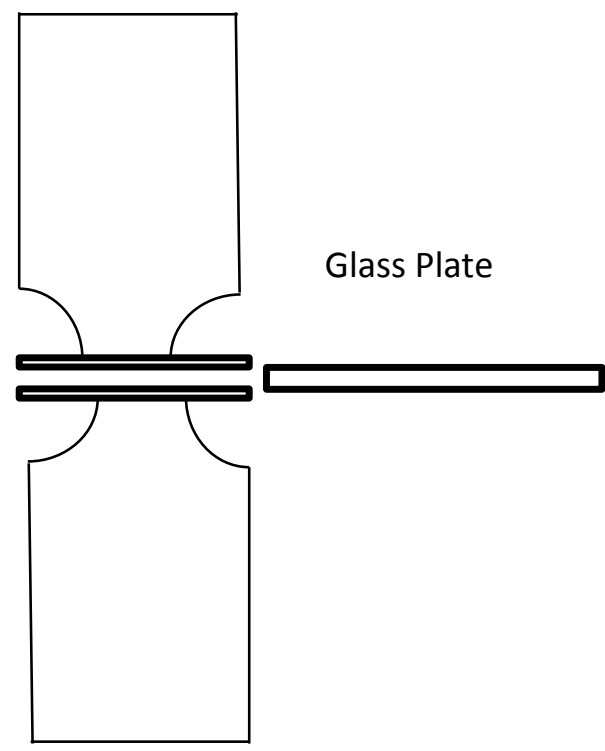

(a)

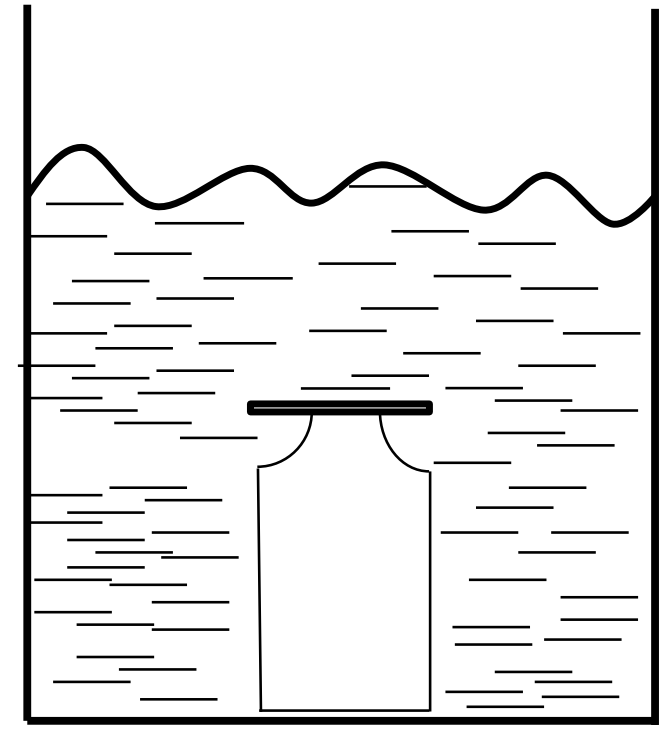

(b)

Figure 1.5. (a) Diffusion in bottles with salt solution; (b) empty bottle in a jar that contains only water

In the first set of experiments (Figure 1.5a), he connected two bottles that contained salt solutions at different concentrations. After several days, he separated the bottles and analyzed the contents to determine any changes. In the second series of experiments (Figure 1.5b), he placed a salt solution of known concentration in a small bottle and placed that bottle in a jar that contained only water. After several days, he took the bottle out of the jar and analyzed its contents. Based on his experiments, Graham concluded that the diffusion process in liquids is slower than diffusion in gases, and the process of diffusion 
becomes even slower as the diffusion progresses. After analyzing the results of his experiments, Graham concluded that" the quantities diffused appear to be closely in proportion to the quantity of salt in the diffusion solution" (Graham, 1850). In other words, "...the flux caused by diffusion is proportional to the concentration difference of the salt."

\subsubsection{Fick's Law}

In 1855, Fick put Graham's experimental results on a quantitative basis. He described the diffusion on the same mathematical basis as Fourier's law of heat conduction or Ohm's Law for electrical conduction. Fick recognized more clearly that diffusion is a dynamic molecular process than Graham did. With this basic hypothesis, Fick developed the laws of diffusion using analogies with Fourier's work. He described a one-dimensional flux, $J_{1}$, by the following equation:

$J_{1}=D \frac{\partial c_{1}}{\partial x}$

where $c_{1}$ is the concentration, and $x$ is the distance. This is Fick's law of diffusion. The quantity D, which Fick referred to as the "constant that depends on the nature of the substance," is also called the diffusion coefficient. Fick used Fourier's development to determine the more general conservation equation:

$\frac{\partial c_{1}}{\partial t}=D\left(\frac{\partial^{2} c_{1}}{\partial x^{2}}+\frac{1}{A} \frac{\partial A}{\partial x} \frac{\partial c_{1}}{\partial x}\right)$

where $A$ is the area and is a constant in a given system. So, Equation (1.2) becomes: 
$\frac{\partial c_{1}}{\partial t}=D\left(\frac{\partial^{2} c_{1}}{\partial x^{2}}\right)$

Equation (1.3) is the basic equation for one-dimensional, unsteady state diffusion. Later, Fick proved his hypothesis that diffusion and thermal conduction could be described by the same equation.

Dense-phase molecular diffusivities are a complex process, and they can be a strong function of composition, temperature, and pressure. Often, they are characterized by the Fick diffusivity, which is a product of the Maxwell-Stefan diffusivity and a thermodynamic, non-ideality factor that is related to the concentration of a chemical species in the medium (E. L. Cussler, 2009). Fick diffusivity is a function of the concentration of the diffusing substance at a given temperature and pressure. This non-ideality is notably present in chemical systems at finite concentrations (Amooghina, et al., 2013; Bouchet et al., 1965; Felder \& Huvard, 1980; Ghoreyshi, et al., 2004; Liu, et al., 2011; Moradi Shehni, et al.,2011; Rehfeldt \& Stichlmair, 2007; Williams \& Cady, 1934).

Multi-component diffusivities, i.e., the simultaneous diffusivities of two or more species in a medium, are far more frequent than the diffusion of a single species (E. L. Cussler \& Peter, 1966).

Binary diffusion can be expressed mathematically as:

$J_{1}=c D_{12} \nabla x_{1}$

Equation (1.4) is another form of Fick's first law of diffusion. The diffusion coefficient $D_{12}$ is called binary diffusivity and $\mathrm{c}$ is the total concentration. There is only one independent 
driving force, $\nabla x_{1}$, and one independent flux, $J_{1}$. However, in a ternary mixture, there are two independent fluxes $\left(J_{1}, J_{2}\right)$ and two independent driving forces $\left(\nabla x_{1}, \nabla x_{2}\right)$. Thus we can write:

$J_{1}=-c D_{11} \nabla x_{1}-c D_{12} \nabla x_{2}$

$J_{2}=-c D_{21} \nabla x_{1}-c D_{22} \nabla x_{1}$

Equations (1.5) and (1.6) show that both fluxes $\left(J_{1}, J_{2}\right)$ depend on both of the independent mole-fraction gradients $\left(\nabla \mathrm{x}_{1}, \nabla \mathrm{x}_{2}\right)$. The $D_{i, j}(i, j=1,2)$ are the multi-component diffusivities. These four diffusivity coefficients are needed to characterize a ternary system (Taylor \& Krishna, 1993).

The focus of our study is to determine the matrix of four diffusivities, i.e., $D_{11}, D_{12}, D_{21}$, and $D_{22}$, in a system that consisted of nitrogen, carbon dioxide, and polystyrene, as shown in Figure 1.6. The diffusion coefficients $D_{11}$ and $D_{22}$ are called main diffusion coefficients also known as self-diffusion coefficients. Whereas the diffusion coefficients $D_{12}$ and $D_{21}$ are called cross-diffusion coefficients also known as mutual diffusion coefficients.

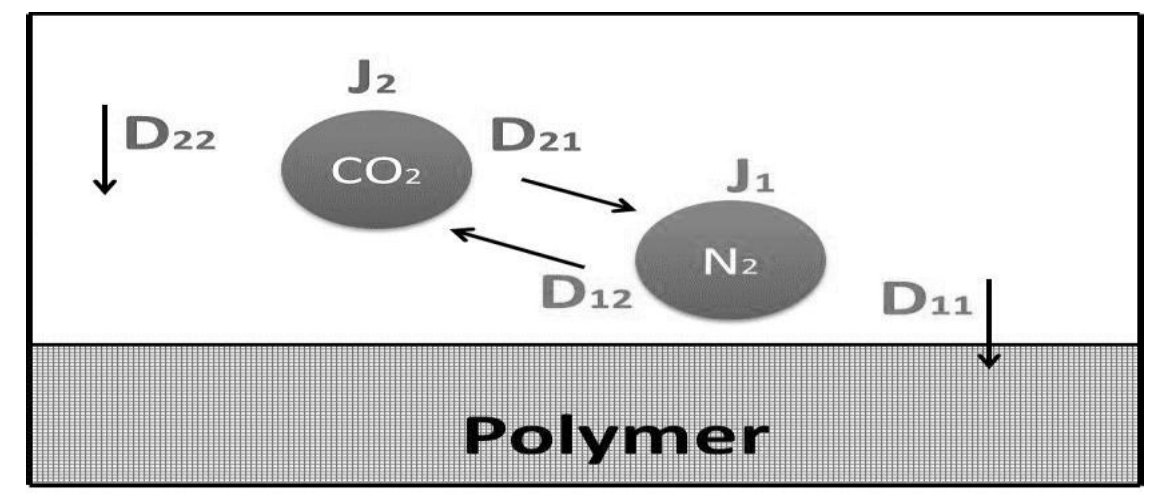

Figure 1.6. Multi-component diffusion in nitrogen, carbon dioxide, and polystyrene 
There are two kinds of blowing agents that are most commonly used namely; Chemical Blowing Agents and Physical Blowing Agents. Chemical blowing agents are chemical compounds that release gases such as nitrogen and carbon dioxide as a result of chemical reactions. Whereas, physical blowing agents are compounds that release gases as a result of physical processes. Examples of widely used physical blowing agents are chlorofluorocarbons and hydrocarbons. Due to the harsh effect of chlorofluorocarbons on environment, their use is prohibited.

However, hydroflourocarbons, hydrocarbons and inert gases such as carbon dioxide and nitrogen can substitute chlorofluorocarbons. Hydroflourocarbons are expensive and flammable. Whereas, hydrocarbons are highly flammable and volatile in nature. The inert gases such as carbon dioxide and nitrogen are benign to the environment.

The polymer industry is gradually introducing carbon dioxide and nitrogen as a safe and low cost physical blowing agent (Kwag, Manke, \& and Gulari, 1999). However, due to the lack of multi-component diffusivity data of carbon dioxide and nitrogen in polymers, the effective design and safe operation of separation and purification processes for polymers is not possible.

Polymer such as polystyrene is colorless, non-toxic, and translucent to transparent solid with a glossy surface, it is mainly used in food packaging and biomedical industry e.g. in petri dishes, injection syringes, electrolyte drips, medical tubing, and in cannula. Where devolatilization and foaming process can be made safe, efficient and economical by using inert gases such as carbon dioxide, and nitrogen. 
The objective is to develop an optimal control framework to determine experimentally the concentration-dependent diffusivities of gas mixtures in polymers, considering first nitrogen and carbon dioxide gases and polystyrene. The optimal control framework developed in this work will produce much-needed diffusivity data, allowing technological advancements in the Canadian polymer industry and others. Multi-component diffusivity data will enable effective design and safe operation of separation and purification processes for polymers and heavy oil. The polymer industry will benefit by using the diffusivity data in engineering, design, and optimization calculations. Regulatory agencies can use the data to establish standards related to polymer products

\subsection{Structure of the Thesis}

This thesis is organized as follows:

Chapter 1 presents the development of the concept of diffusivity from binary to ternary systems.

Chapter 2 presents a detailed literature review of ternary systems, and this review provides the basis for our selection of a system.

Chapter 3 covers the experimental setup, the methods used, and the procedures used.

Chapter 4 covers the development of the mathematical model and the optimal control framework that is used to determine the concentration-dependent, ternary matrix of 
diffusivities. The conditions necessary for solving the mass transfer model is derived. This chapter also includes the computational algorithm.

Chapter 5 presents all the experimental and numerical results. The ternary diffusivity values are calculated. The results are analyzed and discussed.

Chapter 6 summarizes the contribution of this work, and our conclusions and recommendations for future work are presented. 


\section{Literature ReVIeW}

Ternary and higher systems are far more frequent than binary systems. However, the diffusivity data of the former are very limited. In the following treatment, we provide a review of recent studies involving multi-component diffusivity.

Amooghina et al., 2013, developed a new mathematical model to investigate the permeation of a ternary gas mixture across a synthesized composite polydimethylsiloxane/aromatic polyamide membrane. The results showed that the diffusivities of hydrogen and methane increased as the feed temperature and fugacity increased, but the diffusivity of propane decreased. Moreover, increasing the concentration of propane improved the diffusion properties of all of the components. The results demonstrated that considering the concentration-dependent system leads to a small deviation of less than $10 \%$, while the concentration-independent system had a large deviation, ranging from 50 to $100 \%$. In addition, the results indicated that diffusivities of the lighter gases were especially affected by their composition, while solubility had a dominant effect on the diffusivities of the heavier gases.

Liu et al., 2011, developed an empirical method for predicting multi-component, Maxwell Stefan diffusion in ternary systems accounting for friction between the two diffusing 
species. They used an $\mathrm{n}$-hexane-cyclohexane-toluene system, and their results showed that their model described the concentration dependence better than other models.

In a separate investigation, Rehfeldt \& Stichlmair, 2007, studied the diffusivities of liquids, which are of great interest for the calculation and simulation of mass transfer processes. Several prediction models for binary diffusivities can be found in the literature. However, only a few models exist for multi-component systems. Due to lack of data for ternary diffusivities, these models have not been verified for real systems to date. To overcome this limitation, they measured multi-component diffusivities within some concentration range of several ternary systems. Fick diffusivities were transformed to the less concentration-dependent Maxwell-Stefan diffusivities using a thermodynamic correction factor. Four prediction models were tested by comparing their predicted values with the experimental data. In some systems, the predictions of multi-component diffusivities showed promising results. However, the quality of predicted diffusivities depends strongly on an accurate thermodynamic description of the system.

Ghoreyshi et al., 2004 used the Maxwell-Stefan formulation of multi-component diffusivities and the basic postulates of irreversible thermodynamics to develop a general model of membrane transport. In principle, the general model is applicable to any separation process that uses a homogeneous, non-porous membrane as the selectiveseparation barrier. Examples of such processes include dialysis, electrodialysis, reverse osmosis, vapor permeation, and evaporation. The predictive capabilities of the general model were tested for the ethanol-water-silicone rubber system. The results obtained 
indicated that the general model is capable of describing the pervaporation and dialysis performance of the ethanol-water-silicone rubber system with identical sets of concentration-dependent equilibrium and diffusive parameters. They concluded that the concentration dependence of the ternary Maxwell-Stefan diffusivities is described well by a natural extension of the binary relationship to multi-component systems.

Bouchet \& Mevrel, 2007, developed an inverse numerical analysis that permits the extraction of composition-dependent diffusivities for all of the compositions along a diffusivity path. Its application to various diffusion couples at $1100^{\circ} \mathrm{C}$ in a nickel-platinumaluminium alloy showed that, in the composition domain investigated, the direct diffusivities increase as the platinum content increases. A concentration gradient of platinum has no influence on the diffusion of aluminium. The cross coefficient was constant and very small.

J. L. Duda, Ni, \& Vrentas, 1978, showed the general, concentration-dependent behavior of diffusivity. They indicated that diffusivities increase sharply as solvent concentration increases and that they often exhibit maximum values in the concentrated region. At low solvent concentrations, a small increase in the weight fraction of the solvent will cause a very significant increase in the diffusivity (J. L. Duda, 1985). They found that the diffusivity of a solvent in a polymer increases with temperature.

A few publications have reported the concentration dependence of dense-phase gas diffusivities. (Li, Liu, Zhao, \& Yuan, 2009) studied the solubility and diffusivity of carbon dioxide in isotactic polypropylene. The diffusivity showed a weak dependence on 
concentration, and it varied by an order of magnitude (from $10^{-10}$ to $10^{-9} \mathrm{~m}^{2} / \mathrm{s}$ ) in isotactic polypropylene at three different temperatures.

Jitendra, et al., 2009, Kundra, et al., 2011, and Upreti \& Mehrotra, 2000 experimentally determined the composition-dependent diffusivity of nitrogen and carbon dioxide in lowdensity polyethylene, polypropylene, and bitumen. Each time, the researchers found that the gas diffusivity was composition-dependent.

\subsection{Lack of Data for Ternary System}

There are no publications that report experimental diffusivities for ternary systems comprised of two gases and a dense phase. A few experimental studies have investigated only liquid phases. For example, Telis, Murarif. R.C.B.D.L., \& Yamashita, 2004, studied solutions of $\mathrm{NaCl}$ and sucrose for the osmotic pre-treatment of tomato quarters. The maximum loss of moisture occurred when the osmotic treatment was conducted in a more concentrated solution, and this observation was independent of the type of solute. The apparent diffusion coefficients for water, $\mathrm{NaCl}$, and sucrose were calculated at $30 \pm 1^{\circ} \mathrm{C}$, and they were found to be in the range of $3.35 \times 10^{-10}$ to $8.58 \times 10^{-10} \mathrm{~m}^{2} / \mathrm{s}$.

Lin, et al.,, 2009, reported the ternary diffusivities of diethanolamine and $\mathrm{N}$ ethyldiethanolamine in aqueous solutions of these two compounds. The main diffusivities $\left(D_{11}\right.$ and $\left.D_{22}\right)$ and the cross-diffusivities $\left(D_{12}\right.$ and $\left.D_{21}\right)$ were reported as functions of the temperature and concentration of the alkanolamines. They found that the ratio of $D_{12}$ to 
$D_{11}$ was greater than the ratio of $D_{21}$ to $D_{22}$. The diffusion coefficients were on the order of $10^{-9} \mathrm{~m}^{2} / \mathrm{s}$. The researchers also found that the main diffusivities increased as temperature increased at a constant concentration of the solvent. But the diffusivities decreased with the concentration at constant temperature.

Kjetil, et al., 2006, studied a system consisting of toluene, chloroform, and benzene. They determined the concentration-dependent molecular diffusion coefficient to be on the order of $10^{-9} \mathrm{~m}^{2} / \mathrm{s}$.

Our extensive literature survey showed that limited data are available concerning the dependence of diffusivity on concentration. The theoretical prediction of diffusivity relies on the self-diffusivities of the solvent, and these values usually are not available. In addition, the accurate prediction of the concentration-dependent chemical potential of solvents is needed (J. L. Lundberg, et al., 1962; J. L. Lundberg, et al., 1963; J. L. Lundberg, 1964a; J. L. Lundberg, 1964b; J. L. Lundberg, et al., 1960). These limitations necessitate the determination of concentration dependent diffusivities. Table 2.1 summarizes the previous work done by other researchers in determining the diffusivities of carbon dioxide and nitrogen in polystyrene. 
Table 2.1 Diffusion coefficients of carbon dioxide and nitrogen in polystyrene

\begin{tabular}{|c|c|c|c|c|c|}
\hline $\begin{array}{l}\text { Serial. } \\
\text { Number }\end{array}$ & Temperature & $\begin{array}{l}\text { Pressure } \\
\text { (MPa) }\end{array}$ & Polymer (s) & Diffusion Coefficient & Reference \\
\hline 1 & $38{ }^{\circ} \mathrm{C}$ & $9-25$ & $\begin{array}{l}\text { Polystyrene+ } \\
\left(\mathrm{CO}_{2}\right)\end{array}$ & $(0.17-0.48) \times 10^{-6} \mathrm{~cm}^{2} / \mathrm{s}$ & $\begin{array}{l}\text { (Nikitin et al., } \\
2003 \text { ) }\end{array}$ \\
\hline 2 & $50{ }^{0} \mathrm{C}$ & $9-25$ & $\begin{array}{l}\text { Polystyrene+ } \\
\left(\mathrm{CO}_{2}\right)\end{array}$ & $(0.87-0.48) \times 10^{-6} \mathrm{~cm}^{2} / \mathrm{s}$ & $\begin{array}{l}\text { (Nikitin et al., } \\
2003 \text { ) }\end{array}$ \\
\hline 3 & $55^{\circ} \mathrm{C}$ & $9-25$ & $\begin{array}{l}\text { Polystyrene+ } \\
\left(\mathrm{CO}_{2}\right)\end{array}$ & $(1.28-2.18) \times 10^{-6} \mathrm{~cm}^{2} / \mathrm{s}$ & $\begin{array}{l}\text { (Nikitin et al., } \\
2003 \text { ) }\end{array}$ \\
\hline 4 & $65{ }^{0} \mathrm{C}$ & $9-25$ & $\begin{array}{l}\text { Polystyrene+ } \\
\left(\mathrm{CO}_{2}\right)\end{array}$ & $(1.57-3.06) \times 10^{-6} \mathrm{~cm}^{2} / \mathrm{s}$ & $\begin{array}{l}\text { (Nikitin et al., } \\
2003 \text { ) }\end{array}$ \\
\hline 5 & $373.15 \mathrm{~K}$ & $2.34-8.32$ & $\begin{array}{l}\text { Polystyrene+ } \\
\left(\mathrm{CO}_{2}\right)\end{array}$ & $(0.81-1.67) \times 10^{-10} \mathrm{~m}^{2} / \mathrm{s}$ & $\begin{array}{l}\text { (Sato, Takikawa, } \\
\text { Takishima, \& } \\
\text { Masuoka, 2001) }\end{array}$ \\
\hline 6 & $423.15 \mathrm{~K}$ & $2.42-8.31$ & $\begin{array}{l}\text { Polystyrene+ } \\
\left(\mathrm{CO}_{2}\right)\end{array}$ & $(3.01-5.33) \times 10^{-10} \mathrm{~m}^{2} / \mathrm{s}$ & (Sato et al., 2001) \\
\hline 7 & $473.15 \mathrm{~K}$ & $2.52-8.42$ & $\begin{array}{l}\text { Polystyrene+ } \\
\left(\mathrm{CO}_{2}\right)\end{array}$ & $(9.24-9.90) \times 10^{-10} \mathrm{~m}^{2} / \mathrm{s}$ & (Sato et al., 2001) \\
\hline 8 & $200{ }^{\circ} \mathrm{C}$ & $11-12$ & $\begin{array}{l}\text { Polystyrene+ } \\
\text { (CO2) }\end{array}$ & $2.45 \times 10^{-10} \mathrm{~m}^{2} / \mathrm{s}$ & $\begin{array}{l}\text { (Surat, Eita, } \\
\text { Yusuke, Dai, \& } \\
\text { Masahiro, 2004) }\end{array}$ \\
\hline 9 & $373.2-453.2 \mathrm{~K}$ & Up to 17 & $\begin{array}{l}\text { Polystyrene+ } \\
\left(\mathrm{CO}_{2}\right)\end{array}$ & $\begin{array}{l}\text { Solubility } \\
\text { g gas/g polymer } \\
(11.57-6.87) \times 10^{-2}\end{array}$ & $\begin{array}{l}\text { (Sato, Yurugi, } \\
\text { Fujiwara, } \\
\text { Takishima, \& } \\
\text { Masuoka, 1996) }\end{array}$ \\
\hline 11 & $373.2-453.2 \mathrm{~K}$ & Up to 17 & Polystyrene+ (N2) & $(7.15-9.83) \times 10^{-3}$ & (Sato et al., 1996) \\
\hline
\end{tabular}


Our plan is to develop an optimal control framework to determine diffusivity versus concentration surfaces in ternary systems of two gases and a liquid. Therefore, the aim of this study was to develop an optimal control framework novel method for the experimental determination of multi-component diffusivities for a ternary system of two gases and one non-volatile, dense phase. The novel feature of this work is that it allows the natural evolution of multi-component diffusivity verses concentration in agreement with experimental data and subject to the detailed mathematical model. This is an inverse problem that can be solved using the principles of optimal control.

In our study, principles of optimal control is used to extract the optimal, compositiondependent, multi-component diffusivities (system property) as a function of another system property (composition).

\subsection{Optimal Control}

Optimization is a method of finding the conditions that give maximum or minimum value of a function. But, if optimization involves minimization or maximization of a functional subject to some constraint, the decision variable will not be a number, but will be a function. Such problems are called optimal control problems. A functional is defined as a function of several other functions. Optimal control problems involve two kinds of variables: state and control variables. These variables are usually related to each other by a 
set of differential equations. Optimal control theory can be used to solve such problems (Upreti, 2013).

An optimal control technique solves the problem in number of stages, where each stage develops from the preceding stage in prescribed manner. The control variables define the system that governs the advancement of the system from one stage to the next. The state variables describe the behaviour or status of the system at any stage. So, optimal control problem is to find a set of control variables so that the total objective functional over all the stages is optimized subject to a set of constraints (e.g. differential equation) on the control and state variables.

Optimal control determines a control policy for a system that will maximize or minimize a specific performance criterion subject to constrains. Optimal control has applications in many different fields, including aerospace, process control, and engineering. In early 1950s due to the lack of fast computers only simple optimal control problems could be solved. The revolution of the digital computers in the 1950s, allows the application of optimal control theory and methods to solve complex optimal control problems. Many applications of optimal control theory were developed to optimization surfactant flooding process, polymer process, and miscible carbon dioxide process (Ramirez, 1987). Although only initial studies are present, promising advances are expected in the application of optimal control theory.

A branch of mathematics that is useful in solving optimal control problems is the calculus of variations (Denn, 1969; Kirk, 1970; Ray, 1981). Calculus of variations deals with functionals, 
or functions whose independent variables are functions themselves. To solve optimal control problems where the objective is to determine a function that minimize or maximize a specified functional, calculus of variations is a useful technique.

The analogous problem in calculus is to determine a point that yields the minimum or maximum value of a function. The variation plays the same role in determining extreme values of functionals as the differential does in finding maxima and minima of functions. The fundamental theorem used in finding extreme values of a function is the necessary condition that the differential vanishes at an extreme point. In variational problems, the analogous theorem is that the variation must be zero on an extrema.

Consider following simple example of optimal control in which functionals are formed as integrals involving an unknown function and its derivatives:

$I=\int_{t_{o}}^{t_{f}} F(u(t), y(t)) d t$

In Equation $2.1 I$ is a functional of the function $u$ and $y$. It is assumed that $t_{o}$ and $t_{f}$ are initial and final time and are fixed, the end points of the curve are specified as $x_{o}$ and $x_{f}$. The objective is to find the control $u$ for which the functional $I$ has an optimum value subject to the differential equation constraint which is given by

$y=\dot{g}(y, u)$ or $G(y, \dot{y}, u) \equiv-\dot{y}+g(y, u)=0$

with the initial condition 
$y(0)=y_{0}$

At minimum of $I$ the variation of $\delta I$ has to be zero.

$\delta I=\int_{t_{o}}^{t_{f}}\left(F_{y} \delta y+F_{u} \delta u\right) d t=0$

The above equation has to satisfy the differential equation constraint. The $\delta y$ and $\delta u$ cannot be varied arbitrary because they are tied together in the differential objective functional. According to optimal control theory, if the variations are arbitrary their coefficients are individually zero. But in this case this is not possible because the control and the state variable are tied together. This problem can be solved by introducing an undetermined function, $\lambda(t)$, called Lagrange multiplier, in the augmented objective functional defined as

$J \equiv \int_{0}^{t_{f}}[F(y, u)+\lambda G(y, y, u)] d t$

At the optimum the variation of $J$ has to be zero

$$
\delta J=\int_{0}^{t_{f}}\left[F_{y} \delta y+F_{u} \delta u+\lambda\left(G_{y} \delta y+G_{\dot{y}} \delta \dot{y}+G_{u} \delta u\right)+G \delta \lambda\right] d t=0
$$

where the role of the lambda is to untie state variable $y$ from control $u$ by assuming certain values in the time interval $\left[0, t_{f}\right]$. With such values of lambda we are then able to vary $\delta y$ and $\delta u$ arbitrary and independent of each other. This leads to simplified necessary condition for the optimum of $J$ which is equal to the constraind optimum of $I$. The 
condition would add an equation for $\lambda$, the satisfaction of which enables the arbitrary variations in the first place (Upreti, 2013).

To solve an optimal control problem, we must first describe the problem in physical terms, and then translate the physical description into mathematical terms. Once the optimal problem is defined mathematically, we can apply the optimal control theory to the partial differential equations describing the process model. 


\section{EXPERIMENTAL SETUP}

This chapter describes the experimental setup used for determining the concentration dependent multi-component diffusivities of nitrogen and carbon dioxide in polystyrene.

Figure 3.1 shows the experimental setup used to determine the concentration-dependent diffusivities of nitrogen and carbon dioxide in polystyrene. The setup was used to conduct two kinds of experiments, i.e., solubility experiments and diffusion experiments.

The purpose of the solubility experiments was to determine the equilibrium concentration of each gas at the gas-polymer interface as a function of pressure and gas-phase composition. This information provides the boundary condition for the mass transfer model of the diffusion process. The latter is conducted in diffusion experiments to furnish experimental pressure and gas-phase composition as a function of time.

Figure 3.1 shows the main parts of the setup. It consists of a diffusion cell with a concentric, 4-cm-diameter cylindrical slot at the bottom to hold a polymer sample. 


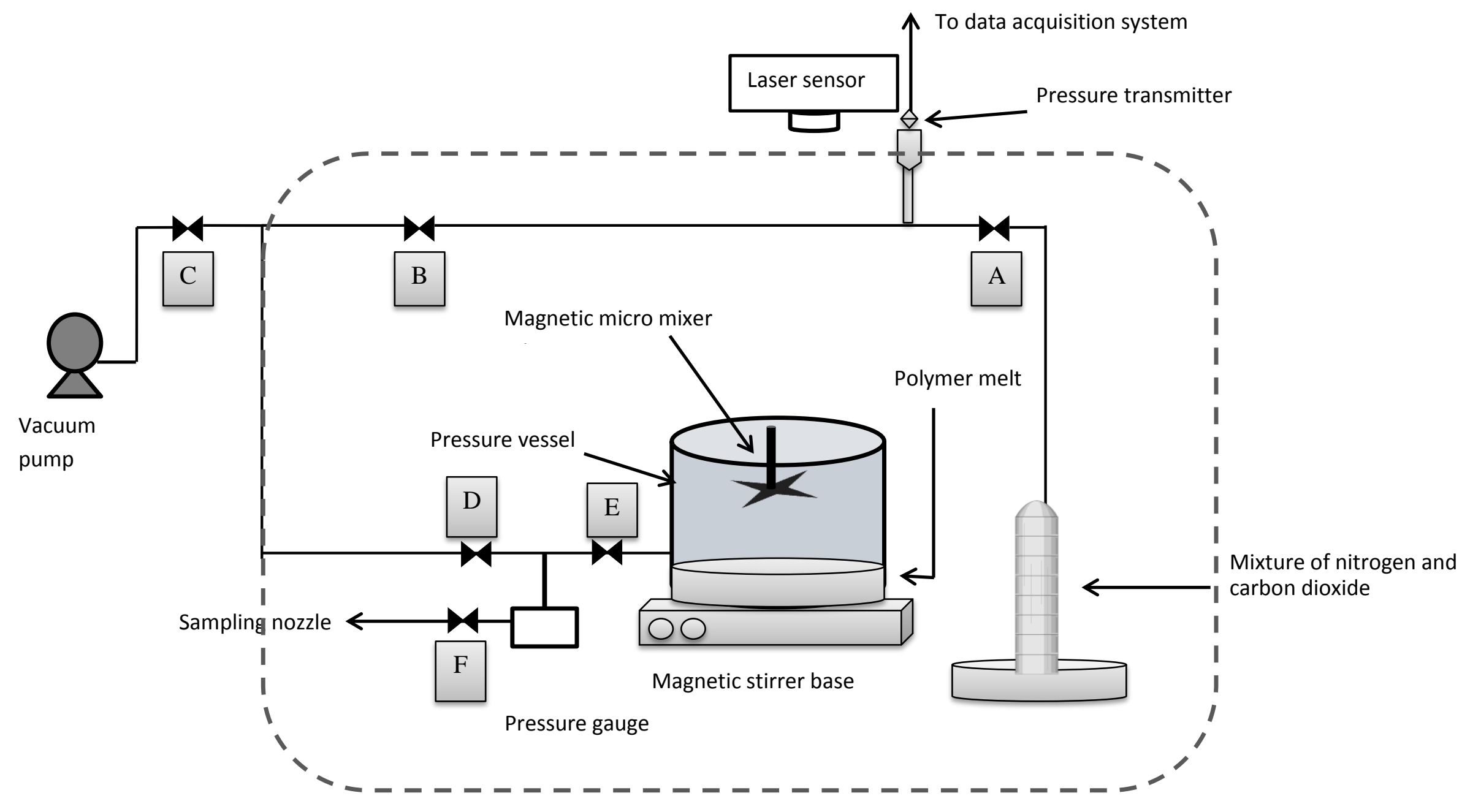

Figure 3.1. Experimental setup 
In an experiment, a mixture of nitrogen and carbon dioxide was allowed to diffuse into the polymer layer inside a closed diffusion cell at a constant temperature.

The dimensions of the diffusion vessel are given in Table 3.1

Table 3.1 Diffusion vessel dimensions and material

\begin{tabular}{|l|l|}
\hline Diameter & $0.04 \mathrm{~m}$ \\
\hline Volume & $32.31 \times 10^{-6} \mathrm{~m}^{3}$ \\
\hline Depth & $0.02 \mathrm{~m}$ \\
\hline $\begin{array}{l}\text { Mass of } \\
\text { Polystyrene }\end{array}$ & $5 \times 10^{-3} \mathrm{~kg}$ \\
\hline $\begin{array}{l}\text { Density of } \\
\text { Polystyrene }\end{array}$ & $1060 \mathrm{~kg} \mathrm{~m}^{-3}$ \\
\hline Carbon & $\begin{array}{l}\text { Obtained from MEGS specialty gases and } \\
\text { equipment, Montreal (99.9\% pure) }\end{array}$ \\
\hline dioxide & $\begin{array}{l}\text { Obtained from MEGS specialty gases and } \\
\text { equipment, Montreal (99.9\% pure) }\end{array}$ \\
\hline Nitrogen & \begin{tabular}{l} 
Sigma Aldrich (99.9\% pure) \\
\hline Polystyrene
\end{tabular} \\
\hline
\end{tabular}

The lid of the diffusion cell had a glass window that allowed a complete view of the surface of the polymer to an external, online, Keyence LKG displacement laser sensor, which tracked the polymer's surface. The laser beam detects any displacement in the thickness of the polymer. The laser sensor has an accuracy of $10 \mu \mathrm{m}$ that could lead to an error of $1.2 \%$. In our experiments, as expected, the laser sensor did not detect any change in the thickness of the polymer because the experiments were done at room temperature $\left(23^{0} \mathrm{C}\right)$ and low pressure. Figure 3.2 shows constant temperature inside the polymer during 
diffusion experiment. Figure 3.3 shows constant thickness of the polymer layer during the diffusion experiment.

To ensure that there is no change in the polymer's temperature during the diffusion process, we inserted a thermocouple in the polymer through the glass lid, as shown in the Figure 3.4(b). The thermocouple used was an H1 Series thermocouple that was obtained from Nanmac Corporation, USA. In our experiments, the thermocouple did not detect any change in the temperature of the polymer. The accuracy of the thermocouple was \pm 0.05 that could generate the error of $0.2 \%$ at $25{ }^{\circ} \mathrm{C}$.

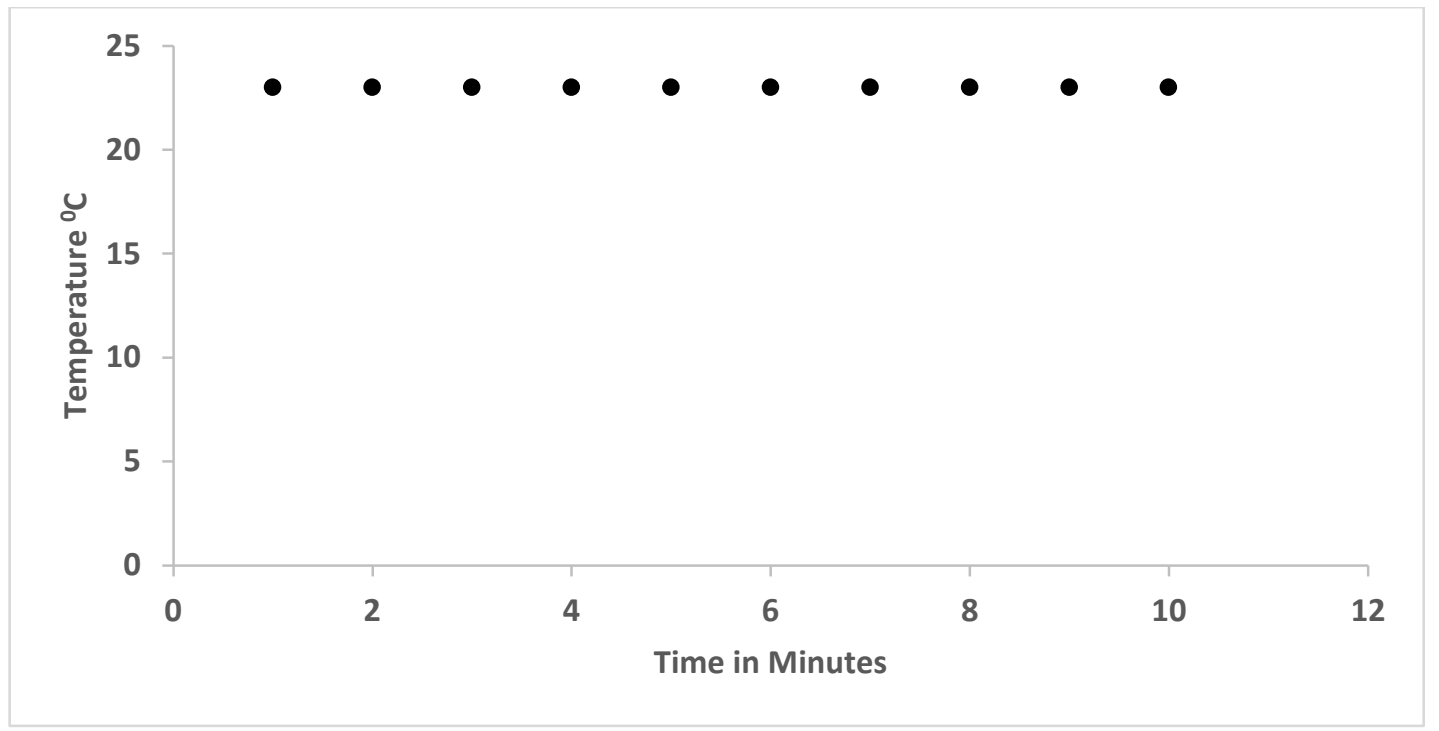

Figure 3.2 Polymer temperature during diffusion experiment 


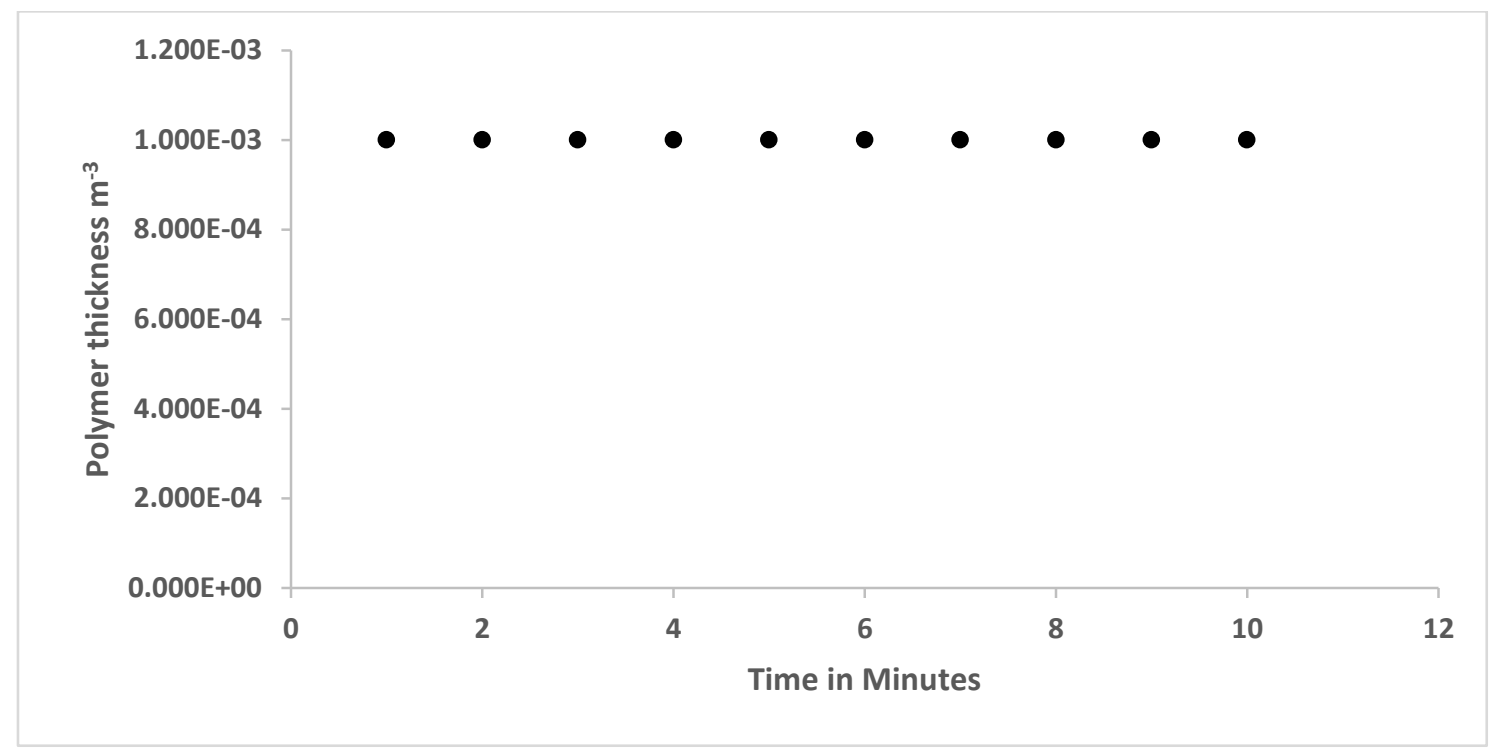

Figure 3.3 Polymer thickness during diffusion experiments

Figure 3.4(a) shows a digital picture of the laser sensor connected to the diffusion system. A Teflon core composite Viton O-ring was used between the lid and the lower part of the diffusion cell to seal the cell. The gas cylinder was used to store the gas that was obtained from an external tank.

The pressure in the diffusion cell was measured by a Paroscientific Digiquartz intelligent pressure transmitter, which was connected to the tube between valves $A$ and $B$ to record the pressure inside the diffusion cell. To maintain isothermal diffusion, the entire setup was placed inside an oven. 
Prior to the experiment, the experimental setup was tested for leaks for $2 \mathrm{~h}$ at the temperature of the experiment by pressurizing it to 1.5 times the pressure used in the experiment. After the system was pressurized, the tests were conducted with valves $A$ and C closed and valve B open. After the test, valve $C$ was opened to depressurize the system, and polystyrene granules were placed in the sample slot and by gradually raising the temperature to form a cylindrical layer. Figure 3.5 shows the polystyrene layer that was formed after the granules were gradually melted and then cooled to $23^{\circ} \mathrm{C}$. The experimental setup was allowed to cool for one day, after which valve $C$ was closed, and the laser sensor was positioned and calibrated to track the movement of the polymer's surface.
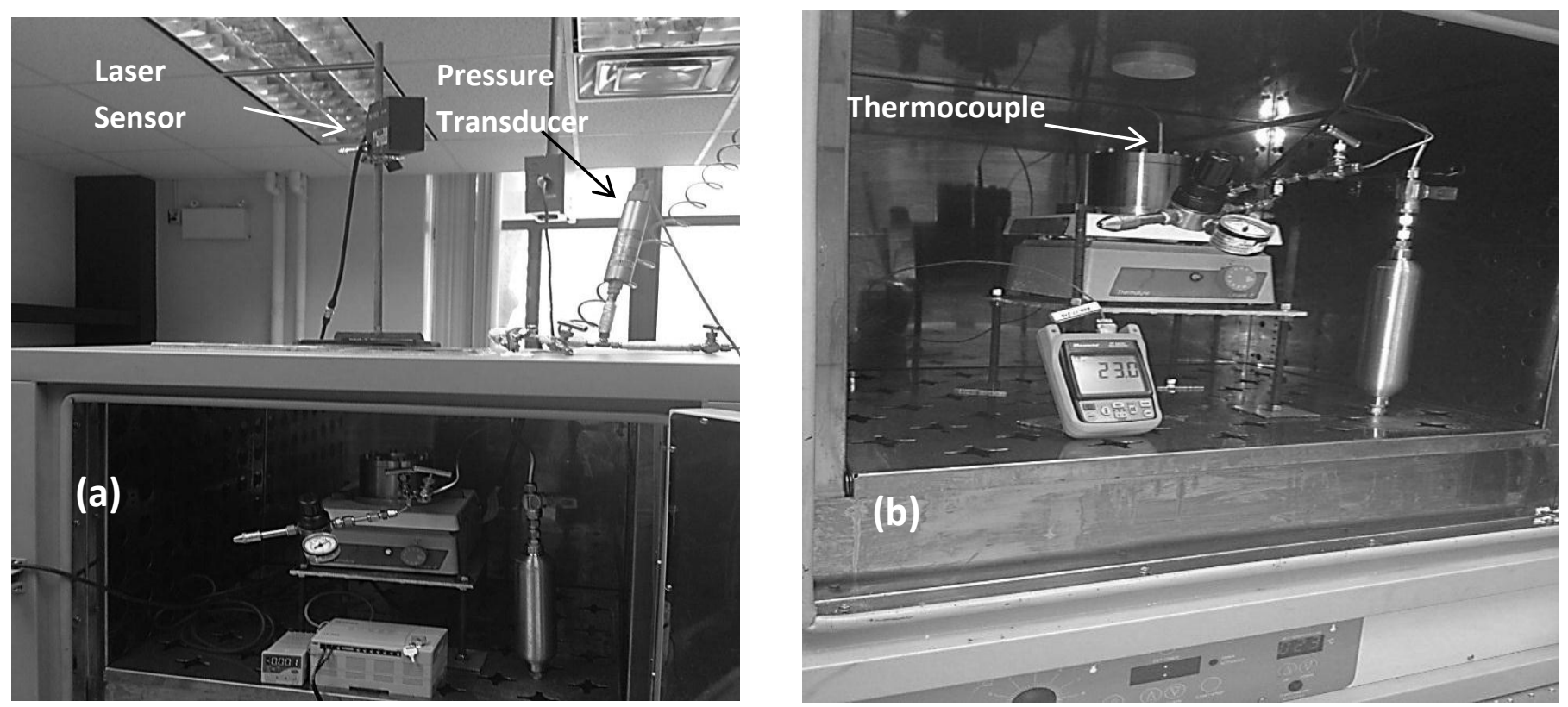

Figure 3.4. (a) Laser sensor; (b) thermocouple 
The experiments were conducted at $23^{\circ} \mathrm{C}$, and the temperature was controlled within $\pm 0.5^{\circ} \mathrm{C}$ of the desired value. The gaseous mixture was introduced and only valve $\mathrm{E}$ and $\mathrm{C}$ were closed. Initial sample of the gaseous mixture was withdrawn from valve $F$. Valve $F$ is then closed and the experiment was initiated by introducing the gas quickly above the polymer's surface inside the diffusion cell by simultaneously opening valve $E$ and closing valve $A$. The pressure inside the cell was recorded as the gas diffused gradually into the polymer layer. The pressure sensor had a resolution of $\pm 0.006 \mathrm{kPa}$. To withdraw final sample valve $D$ is closed and the sample is withdrawn from valve $F$.

To determine gas solubility and diffusion, the experiment was conducted until no further reduction in the pressure could be discerned. That was the time when the mass fraction of the gas in the polymer sample tended to have a uniform equilibrium value. The experiment was terminated by gradually opening valve $C$ to release the gas. After the completion of each run, the gas was removed from the apparatus by a vacuum pump. After the vacuum pump had removed the gas, it was turned off, and the pressure was allowed to stabilize. The vacuum pump was operated a few times to ensure that all of the gas in the polymer and in the gas phase had been removed. Then, the second run was begun using the same steps described above.

A magnetic micro mixing fan was affixed beneath the lid of the diffusion cell to homogenize the gas phase. The minimum speed that could be achieved for magnetic micro mixing was 30 revolutions per minute and this speed was used throughout our experiments. Another reason for rotating the fan at lower speed is to avoid any convection and bulk motion. Also 
at higher fan speed there would be work done by the fan inside the pressure vessel that could increase the temperature of the gas phase and the polymer. This magnetic micro mixing fan obviates the need for an additional mass transfer model for the gas phase. The speed of the magnetic micro mixing fa Figure 3.6 shows a schematic diagram of the fan, which was made by gluing aluminum strips to the bar of a magnetic stirrer at an angle of $45^{\circ}$. The magnetic micro mixing fan was attached to the base of the lid of the cell.

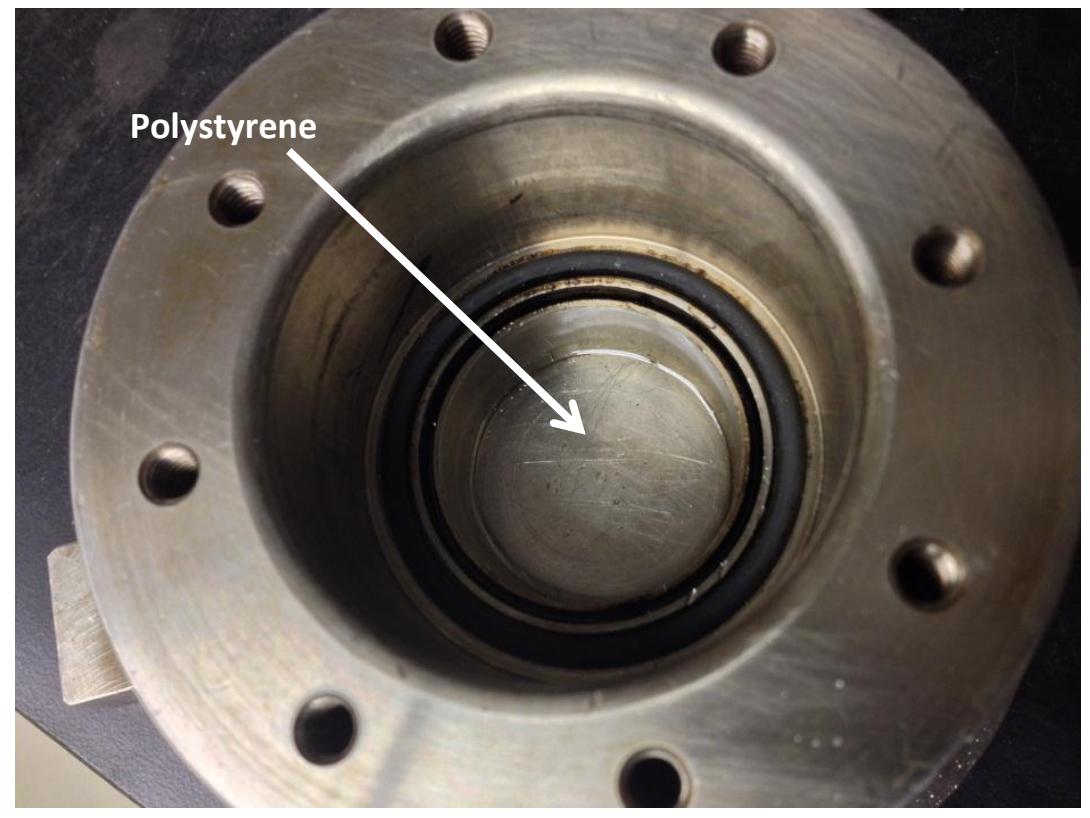

Figure 3.5. Polystyrene layer with uniform thickness 


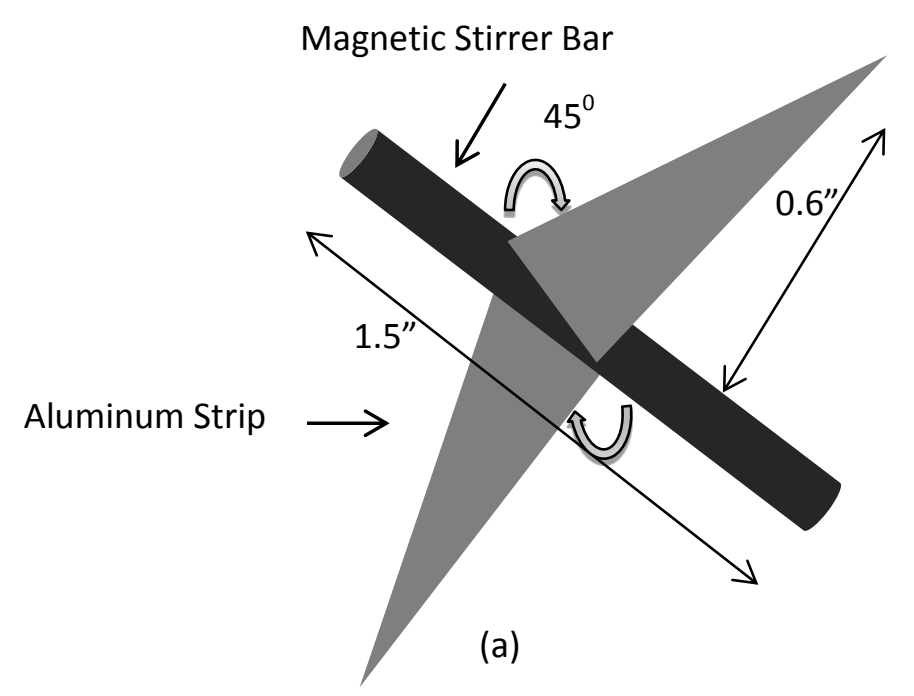

Figure 3.6. Magnetic mixer

\subsection{Solubility Experiments}

Different initial ratios of the two gases and different initial pressures were used, and the diffusion of nitrogen and carbon dioxide in polystyrene was conducted until there was no detectable change in pressure inside the diffusion cell, which took an extended period of time (1-2 days). The final pressures were recorded, and gas-phase samples were extracted at the beginning and end of the experiment and analyzed using a gas chromatograph to determine the initial and final gas compositions. Five solubility experiments were conducted with different initial compositions in the gas phase. The plots of the solubility experiments are given in Appendix A. 
The purpose of these experiments was to determine the interfacial (equilibrium) concentration of nitrogen and carbon dioxide at the gas-polymer interface as a function of pressure and gas-phase composition:

$\omega_{i}^{*}=\omega_{i}^{*}\left(P, y_{1}\right) \quad$ where $i=1,2$

The $\omega_{i}^{*}$ s formed the boundary conditions in the mass transfer model to be used for the determination of the concentration-dependent, multi-component diffusivities of the gases in the polymer. These masses provided the solubility or $\omega_{i}^{*} s$ at the final pressures and gasphase compositions.

\subsection{Diffusion Experiments}

With a fixed initial ratio of the masses of the two gases and a fixed initial pressure, the diffusion process was conducted for $2,4,6,8$, and 10 minutes. The composition of the gas phase $\left(y_{1}\right)$ was determined at the beginning and the end of each experiment using a gas chromatograph.

The purpose of these experiments was to obtain data for pressure and gas-phase composition as a function of time, i.e., $P(t)$ and $y_{1}(t)$. These data provided:

1. The experimental values of the masses of the two gases absorbed in the polymer as a function of time. 
2. The calculated counterparts of the above masses given by the mass transfer model, which had:

(a) Boundary conditions that were obtained from solubility experiments:

$\omega_{i}^{*}=\omega_{i}^{*}\left(P, y_{1}\right) \quad$ for $i=1,2$

(b) Composition-dependent diffusivities of each gas in polymer:

$D_{i j}=D_{i j}\left(\omega_{1}, \omega_{2}\right) \quad$ for $i, j=1,2$

\subsection{Analysis of Gas Phase Composition}

The gas-phase composition during the diffusion and solubility experiments was analyzed by a gas chromatograph. Now, the gas chromatograph is introduced briefly, including how it works, how the samples were extracted during the solubility and diffusion experiments, the column used in this study, and the gas chromatography method that was used in the analysis of the composition of the gas phase. A succinct introduction of the gas chromatograph (GC) follows.

\subsubsection{Gas Chromatography}

Chromatography is the separation of a mixture of compounds (solutes) into separate components, making it easier to identify (qualitate) and measure (quantitate) each component. GC is one of several chromatographic techniques. It is appropriate for 
analyzing $10-20 \%$ of all known compounds. To be suitable for GC analysis, a compound must have sufficient volatility and thermal stability. If all or some of a compound's molecules are in the gas phase at $400-450{ }^{\circ} \mathrm{C}$ or below and they do not decompose at these temperatures, the compound probably can be analyzed by GC.

\subsubsection{Chromatogram}

The size of the peak corresponds to the amount of the compound of interest in the sample. As the amount of the compound of interest increases in the samples being analyzed, larger peaks are attained for that compound. Retention time is the time it takes for a compound to travel through the column. If the column and all operating conditions are kept constant, a given compound will always have the same retention time.

Peak size and retention time are used for quantitative and qualitative analyses of a compound, respectively. However, the identification of a compound cannot be determined solely by its retention time. A known amount of an authentic, pure sample of the compound must first be analyzed to determine its retention time and peak size. Then, this value is compared to the results from an unknown sample to determine whether the target compound is present (by comparing retention times) and in what quantity (by comparing peak size).

In this study, thermal conductivity detector was used. Thermal conductivity detector relies on the thermal conductivity of matter passing around a tungsten-rhenium filament with a 
current traveling through it. In this set up helium is used as a carrier gas because of their relatively high thermal conductivity which keep the filament cool and maintain uniform resistivity and electrical efficiency of the filament. However, when analyte molecules elute from the column, mixed with carrier gas, the thermal conductivity decreases and this causes a detector response. The response is due to the decreased thermal conductivity causing an increase in filament temperature and resistivity resulting in fluctuations in voltage. Detector sensitivity is proportional to filament current while it is inversely proportional to the immediate environmental temperature of that detector as well as flow rate of the carrier gas.

A Varian gas chromatograph, model CP3800, with Galaxie V1.9 software, was used to determine the amounts of carbon dioxide and nitrogen in the sample obtained from the diffusion cell. The column HP-Plot Q, obtained from Agilent Technologies Canada, was used for the detection of eluents from the gas chromatograph. The inner diameter of the column was $0.32 \mathrm{~mm}$, and the length of the column was $15 \mathrm{~m}$. The stationary phase in the column was polystyrene divinyl benzene.

\subsubsection{Sampling}

A gas-tight, high-performance, micro syringe with a volume of $1,000 \mu \mathrm{L}$, obtained from Sigma-Aldrich, was used to draw the samples from the sampling nozzle. The sample in the syringe was injected manually into the gas chromatograph column for detection. 
Before we used the GC to determine the experimental masses of nitrogen and carbon dioxide absorbed in the polystyrene, we ran pure samples of nitrogen and carbon dioxide with different initial volumes. Then the areas under the peaks were plotted against the volume of the samples. The best straight-line fit of the data was determined and for use in determining the composition of these two gases in the unknown sample. The curve-fitting plots are provided in Appendix B.

During the solubility and diffusion experiments, $200 \mu \mathrm{L}$ samples were injected manually through the GC sample inlet at $240^{\circ} \mathrm{C}$. The retention time was determined by a thermal conductivity detector that was maintained at $240^{\circ} \mathrm{C}$.

Helium was used as the carrier gas (mobile phase). The flow rate of the mobile phase was $8.6 \mathrm{~mL} / \mathrm{min}$. The oven that housed the GC column was maintained at $35^{\circ} \mathrm{C}$.

Using the measured composition of the gas phase, we calculated the experimental mass of each gas absorbed in the polystyrene at the time when the samples were extracted. The detailed calculation is given in Appendix B. 


\section{THEORY and COMPUTATION}

This chapter presents the development of optimal control framework to determine concentration dependent multicomponent diffusivities of two gases in a non-volatile dense phase, which is the primary objective of this study. Interfacial gas mass fractions of two gases versus time are used as control in this optimal control problem. The optimal diffusivities are then determined that minimises the error between the experimental the calculated gas mass absorbed.

The optimal control framework to determine the concentration dependent multicomponent diffusivities is based on detailed mass transfer model, which comprises continuity equation of diffusion. Optimal control principles are applied to derive necessary conditions for which the error between the experimental and calculated gas mass absorbed is minimum. A numerical algorithm is developed to estimate the optimal ternary diffusivities.

\subsection{Mass Transfer Model}

The mathematical model was based on the following assumptions:

1. Mass transfer is along the depth of the dense phase. 
The polymer is in the pressure-decay vessel and only its surfaces are exposed to the diffusing gases. The other three sides of the polymer are adhered to the base or the walls of the pressure-decay vessel. Based on these facts, it can be assumed that the diffusion of the gases in the polymer melt is only in the downward direction (z-direction). Hence, it is a unidirectional diffusion process.

2. No chemical reactions occur in the pressure vessel.

The absorption of the gases in the polymer melt is purely a physical phenomenon. There are no reactions among carbon dioxide, nitrogen, and the polymer melt at the temperature and pressure of the experiment.

3. Diffusion occurs at constant temperature.

While the diffusion of the gases is occurring, the temperature of the pressure-decay system and its components does not change. (This assumption inherently means that any thermal energy released during the diffusion is dissipated instantaneously to the surroundings

4. There is no convection in the dense, non-volatile phase.

The rotation of magnetic micro mixer was kept low at $30 \mathrm{rpm}$. Hence, it is assumed that there is no diffusion due to convection or bulk motion in the gas phase due to the rotation of the magnetic micro mixer inside the pressure-decay vessel. Also, at this low rotational speed, the work done by the magnetic micro mixer does not affect the temperature inside the pressure-decay vessel.

5. The wall effects are negligible.

It is assumed that the permeating gases (carbon dioxide and nitrogen) move only along the z-direction i.e., the depth of the polymer. 
6. The pressure decay is solely due to the diffusion of the gases and there is no leakage.

Before the experiment was started, it was ensured that the pressure-decay equipment had no leaks. Hence, it is assumed that the decrease in the pressure is solely due to diffusion.

7. The gas phase is homogenous at all times.

Since the magnetic micro mixer was constantly moving at slow speed above the surface of the polymer. So, it is assumed that the gas phase is homogenous at all times.

Figure 4.1 shows the diffusion of nitrogen and carbon dioxide in polystyrene. Dark colored circles represent carbon dioxide and the white color circles represent nitrogen. The two gases diffuse in the polymer of depth of $z$. The diffusivity of the two gases is a function of the mass fraction of the two gases $D_{i, j}\left(\omega_{i}, \omega_{j}\right)$.

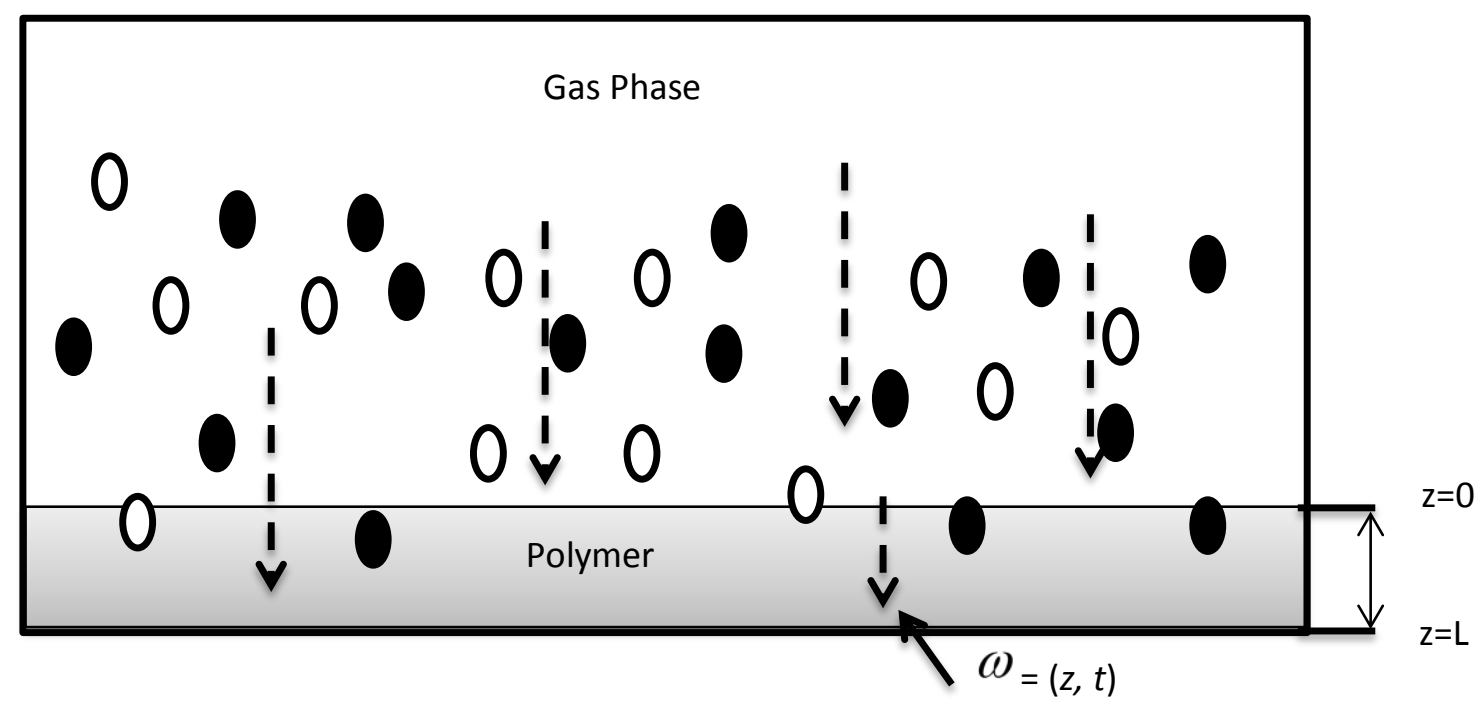

Figure 4.1. Diffusion of two gases in an underlying dense, non-volatile phase
Carbon dioxide $\mathrm{O}$ Nitrogen
$\mathrm{z}=\mathrm{L}=$ length 


\subsection{Theoretical Model Development}

Considering the assumptions mentioned above, the mass transfer model is presented below. We use subscripts ' 1 ' and ' 2 ' for the nitrogen and carbon dioxide respectively, and ' 3 ' for the non-volatile phase. For the first gas:

$\frac{\partial \omega_{1}}{\partial t}+\frac{\partial N_{1}}{\partial z}=0$,

where $\omega_{1}$ is the mole fraction of the first gas, and $N_{1}$ is the mass flux of that gas, both in the underlying layer of the dense, non-volatile phase; the flux is given by:

$N_{1}=\omega_{1} N_{1}+\omega_{1} N_{2}+\omega_{1} N_{3}+J_{1}$

where $N_{2}$ and $N_{3}$ are the mass flux of the second gas and the dense non-volatile phase, respectively. Since $N_{3}$ is zero, Equation (4.2) becomes:

$$
N_{1}=\omega_{1} N_{1}+\omega_{1} N_{2}+J_{D}
$$

where $\mathbf{J}_{1}$ is the diffusive flux for the first gas, and it is given by:

$$
\begin{aligned}
& J_{D}=-D_{11} \frac{\partial \omega_{1}}{\partial z}-D_{12} \frac{\partial \omega_{2}}{\partial z} \\
& N_{1}=\left(\frac{1}{1-\omega_{1}}\right)\left(\left(\omega_{1} N_{2}\right)-\left(D_{11} \frac{\partial \omega_{1}}{\partial z}+D_{12} \frac{\partial \omega_{2}}{\partial z}\right)\right)
\end{aligned}
$$

Similarly for the second gas, we have

$$
N_{2}=\left(\frac{1}{1-\omega_{2}}\right)\left(\left(\omega_{2} N_{1}\right)-\left(D_{22} \frac{\partial \omega_{2}}{\partial z}+D_{21} \frac{\partial \omega_{1}}{\partial z}\right)\right)
$$

Substituting Equation (4.6) in Equation (4.5), we get: 


$$
N_{1}=\left(\frac{1}{1-\omega_{1}}\right)\left\{\omega_{1}\left(\frac{1}{1-\omega_{2}}\left(\omega_{2} N_{1}-D_{22} \frac{\partial \omega_{2}}{\partial z}-D_{21} \frac{\partial \omega_{1}}{\partial z}\right)-\left(D_{11} \frac{\partial \omega_{1}}{\partial z}+D_{12} \frac{\partial \omega_{2}}{\partial z}\right)\right)\right\}
$$

Simplifying for $N_{1}$ we get

$$
\begin{aligned}
& N_{1}=\left\{\left(\frac{\omega_{1} \omega_{2}}{\left(1-\omega_{1}\right)\left(1-\omega_{2}\right)} N_{1}-\frac{\omega_{1}}{\left(1-\omega_{1}\right)\left(1-\omega_{2}\right)}\left(D_{22} \frac{\partial \omega_{2}}{\partial z}+D_{21} \frac{\partial \omega_{1}}{\partial z}\right)-\frac{1}{\left(1-\omega_{1}\right)}\left(D_{11} \frac{\partial \omega_{1}}{\partial z}+D_{12} \frac{\partial \omega_{2}}{\partial z}\right)\right)\right\} \\
& N_{1}=-\left\{\frac{1}{\left.1-\frac{\omega_{1} \omega_{2}}{\left(1-\omega_{1}\right)\left(1-\omega_{2}\right)}\left(\frac{\omega_{1}}{\left(1-\omega_{1}\right)\left(1-\omega_{2}\right)}\left(D_{22} \frac{\partial \omega_{2}}{\partial z}+D_{21} \frac{\partial \omega_{1}}{\partial z}\right)+\frac{1}{\left(1-\omega_{1}\right)}\left(D_{11} \frac{\partial \omega_{1}}{\partial z}+D_{12} \frac{\partial \omega_{2}}{\partial z}\right)\right)\right\}}\right. \\
& N_{1}=-\left\{\frac { ( 1 - \omega _ { 1 } ) ( 1 - \omega _ { 2 } ) } { ( 1 - \omega _ { 1 } ) ( 1 - \omega _ { 2 } ) - \omega _ { 1 } \omega _ { 2 } } \left(\frac{1}{\left(1-\omega_{1}\right)\left(1-\omega_{2}\right)}\left(D_{22} \frac{\partial \omega_{2}}{\partial z}+D_{21} \frac{\partial \omega_{1}}{\partial z}\right)+\left(D_{11} \frac{\partial \omega_{1}}{\partial z}+D_{12} \frac{\partial \omega_{2}}{\partial z}\right)\right.\right. \\
& N_{1}=-\left\{\frac{\left(1-\omega_{1}\right)}{\left(1-\omega_{1}-\omega_{2}\right)}\left(\frac{\omega_{1}}{\left(1-\omega_{2}\right)}\left(D_{22} \frac{\partial \omega_{2}}{\partial z}+D_{21} \frac{\partial \omega_{1}}{\partial z}\right)+\left(D_{11} \frac{\partial \omega_{1}}{\partial z}+D_{12} \frac{\partial \omega_{2}}{\partial z}\right)\right)\right\}
\end{aligned}
$$

Now substituting Equation (4.11) into Equation (4.1), we get

$$
\frac{\partial \omega_{1}}{\partial t}=\frac{\partial}{\partial z}\left\{\frac{\left(1-\omega_{2}\right)}{\left(1-\omega_{1}-\omega_{2}\right)}\left(\frac{\omega_{1}}{\left(1-\omega_{2}\right)}\left(D_{22} \frac{\partial \omega_{2}}{\partial z}+D_{21} \frac{\partial \omega_{1}}{\partial z}\right)+\left(D_{11} \frac{\partial \omega_{1}}{\partial z}+D_{12} \frac{\partial \omega_{2}}{\partial z}\right)\right)\right\}
$$

or

$$
\frac{\partial \omega_{1}}{\partial t}=\frac{\partial}{\partial z}\left[\frac{\omega_{1}}{\left(1-\omega_{1}-\omega_{2}\right)}\left(D_{22} \frac{\partial \omega_{2}}{\partial z}+D_{21} \frac{\partial \omega_{1}}{\partial z}\right)\right]+\frac{\partial}{\partial z}\left[\left(\frac{\left(1-\omega_{2}\right)}{\left(1-\omega_{1}-\omega_{2}\right)}\left(D_{11} \frac{\partial \omega_{1}}{\partial z}+D_{12} \frac{\partial \omega_{2}}{\partial z}\right)\right)\right]
$$


Taking derivative w.r.t $\frac{\partial}{\partial z}$ of R.H.S of Equation (4.13) we get the model for change in mass fraction of gas 1 w.r.t time.

Taking derivative of the first term on the R.H.S of Equation (4.13)

$$
\begin{aligned}
& \frac{\partial}{\partial z}\left[\frac{\omega_{1}}{\left(1-\omega_{1}-\omega_{2}\right)}\left(D_{22} \frac{\partial \omega_{2}}{\partial z}+D_{21} \frac{\partial \omega_{1}}{\partial z}\right)\right]= \\
& \left\{\left(\frac{\omega_{1}}{\left(1-\omega_{1}-\omega_{2}\right)^{2}}+\frac{1}{1-\omega_{1}-\omega_{2}}\right) \frac{\partial \omega_{1}}{\partial z}+\left(\frac{\omega_{1}}{\left(1-\omega_{1}-\omega_{2}\right)^{2}}\right) \frac{\partial \omega_{2}}{\partial z}\right\}\left(D_{22} \frac{\partial \omega_{2}}{\partial z}+D_{21} \frac{\partial \omega_{1}}{\partial z}\right)+ \\
& \frac{\omega_{1}}{\left(1-\omega_{1}-\omega_{2}\right)}\left[\begin{array}{l}
\frac{\partial \omega_{2}}{\partial z} \frac{\partial D_{22}}{\partial \omega_{1}} \frac{\partial \omega_{1}}{\partial z}+D_{22}\left(\frac{\partial \omega_{2}}{\partial z}\right)^{2}+\frac{\partial \omega_{2}}{\partial z} \frac{\partial D_{22}}{\partial \omega_{2}} \frac{\partial \omega_{2}}{\partial z}+D_{21}\left(\frac{\partial \omega_{1}}{\partial z}\right)^{2}+ \\
\frac{\partial \omega_{1}}{\partial z} \frac{\partial D_{21}}{\partial \omega_{1}} \frac{\partial \omega_{1}}{\partial z}+\frac{\partial \omega_{1}}{\partial z} \frac{\partial D_{21}}{\partial \omega_{2}} \frac{\partial \omega_{2}}{\partial z}
\end{array}\right]
\end{aligned}
$$

Taking derivative of the second term on the R.H.S of Equation (4.13)

$$
\begin{aligned}
& \frac{\partial}{\partial z}\left[\left(\frac{\left(1-\omega_{2}\right)}{\left(1-\omega_{1}-\omega_{2}\right)}\left(D_{11} \frac{\partial \omega_{1}}{\partial z}+D_{12} \frac{\partial \omega_{2}}{\partial z}\right)\right)\right]= \\
& {\left[\left(\frac{1-\omega_{2}}{\left(1-\omega_{1}-\omega_{2}\right)^{2}}\right) \frac{\partial \omega_{1}}{\partial z}+\left(\frac{1-\omega_{2}}{\left(1-\omega_{1}-\omega_{2}\right)^{2}}-\frac{1}{1-\omega_{1}-\omega_{2}}\right) \frac{\partial \omega_{2}}{\partial z}\right]\left(D_{11} \frac{\partial \omega_{1}}{\partial z}+D_{12} \frac{\partial \omega_{2}}{\partial z}\right)+} \\
& \frac{\left(1-\omega_{2}\right)}{\left(1-\omega_{1}-\omega_{2}\right)}\left[\begin{array}{l}
\left.D_{11}\left(\frac{\partial \omega_{1}}{\partial z}\right)^{2}+\frac{\partial \omega_{1}}{\partial z} \frac{\partial D_{11}}{\partial \omega_{1}} \frac{\partial \omega_{1}}{\partial z}+\frac{\partial \omega_{1}}{\partial z} \frac{\partial D_{11}}{\partial \omega_{2}} \frac{\partial \omega_{2}}{\partial z}+\frac{\partial \omega_{2}}{\partial z} \frac{\partial D_{12}}{\partial \omega_{1}} \frac{\partial \omega_{1}}{\partial z}+\right] \\
D_{21}\left(\frac{\partial \omega_{2}}{\partial z}\right)^{2}+\frac{\partial \omega_{2}}{\partial z} \frac{\partial D_{12}}{\partial \omega_{2}} \frac{\partial \omega_{2}}{\partial z}
\end{array}\right]
\end{aligned}
$$

Substituting Equation (4.14) and Equation (4.15) in Equation (4.13) we get, 


$$
\begin{aligned}
& \frac{\partial \omega_{1}}{\partial t}=\left\{\left(\frac{\omega_{1}}{\left(1-\omega_{1}-\omega_{2}\right)^{2}}+\frac{1}{1-\omega_{1}-\omega_{2}}\right) \frac{\partial \omega_{1}}{\partial z}+\left(\frac{\omega_{1}}{\left(1-\omega_{1}-\omega_{2}\right)^{2}}\right) \frac{\partial \omega_{2}}{\partial z}\right\}\left(D_{22} \frac{\partial \omega_{2}}{\partial z}+D_{21} \frac{\partial \omega_{1}}{\partial z}\right)+ \\
& \frac{\omega_{1}}{\left(1-\omega_{1}-\omega_{2}\right)}\left[\frac{\partial \omega_{2}}{\partial z} \frac{\partial D_{22}}{\partial \omega_{1}} \frac{\partial \omega_{1}}{\partial z}+D_{22}\left(\frac{\partial^{2} \omega_{2}}{\partial z^{2}}\right)+\frac{\partial \omega_{2}}{\partial z} \frac{\partial D_{22}}{\partial \omega_{2}} \frac{\partial \omega_{2}}{\partial z}+D_{21}\left(\frac{\partial \omega_{1}}{\partial \omega_{1}}\right)+\right] \\
& \left.+\left[\left(\frac{1-\omega_{1}}{\partial z}+\frac{\partial \omega_{1}}{\partial z} \frac{\partial D_{21}}{\partial \omega_{2}} \frac{\partial \omega_{2}}{\partial z}\right)\right] \frac{\partial \omega_{1}}{\partial z}+\left(\frac{1-\omega_{2}}{\left(1-\omega_{1}-\omega_{2}\right)^{2}}-\frac{1}{1-\omega_{1}-\omega_{2}}\right) \frac{\partial \omega_{2}}{\partial z}\right]\left(D_{11} \frac{\partial \omega_{1}}{\partial z}+D_{12} \frac{\partial \omega_{2}}{\partial z}\right)+ \\
& \frac{\left(1-\omega_{2}\right)}{\left(1-\omega_{1}-\omega_{2}\right)}\left[\begin{array}{l}
\left.D_{11}\left(\frac{\partial^{2} \omega_{1}}{\partial z^{2}}\right)+\frac{\partial \omega_{1}}{\partial z} \frac{\partial D_{11}}{\partial \omega_{1}} \frac{\partial \omega_{1}}{\partial z}+\frac{\partial \omega_{1}}{\partial z} \frac{\partial D_{11}}{\partial \omega_{2}} \frac{\partial \omega_{2}}{\partial z}+\frac{\partial \omega_{2}}{\partial z} \frac{\partial D_{12}}{\partial \omega_{1}} \frac{\partial \omega_{1}}{\partial z}+\right] \\
D_{21}\left(\frac{\partial^{2} \omega_{2}}{\partial z^{2}}\right)+\frac{\partial \omega_{2}}{\partial z} \frac{\partial D_{12}}{\partial \omega_{2}} \frac{\partial \omega_{2}}{\partial z}
\end{array}\right]
\end{aligned}
$$

Or

$$
\begin{aligned}
& f_{1}=\frac{\partial \omega_{1}}{\partial t}=\left\{\begin{array}{l}
\left(\frac{\omega_{1}}{\left(1-\omega_{1}-\omega_{2}\right)^{2}}+\frac{1}{1-\omega_{1}-\omega_{2}}\right)\left(D_{22} \frac{\partial \omega_{2}}{\partial z}+D_{21} \frac{\partial \omega_{1}}{\partial z}\right)+ \\
\left(\frac{1-\omega_{2}}{\left(1-\omega_{1}-\omega_{2}\right)^{2}}\right)\left(D_{11} \frac{\partial \omega_{1}}{\partial z}+D_{12} \frac{\partial \omega_{2}}{\partial z}\right)
\end{array}\right\} \frac{\partial \omega_{1}}{\partial z}+ \\
& \left\{\begin{array}{l}
\left(\frac{\omega_{1}}{\left(1-\omega_{1}-\omega_{2}\right)^{2}}\right)\left(D_{22} \frac{\partial \omega_{2}}{\partial z}+D_{21} \frac{\partial \omega_{1}}{\partial z}\right)+\left(\frac{1-\omega_{2}}{\left(1-\omega_{1}-\omega_{2}\right)^{2}}-\frac{1}{1-\omega_{1}-\omega_{2}}\right) \\
\left(D_{11} \frac{\partial \omega_{1}}{\partial z}+D_{12} \frac{\partial \omega_{2}}{\partial z}\right)
\end{array}\right\} \frac{\partial \omega_{2}}{\partial z}+ \\
& \left\{\begin{array}{l}
D_{21} \frac{\omega_{1}}{\left(1-\omega_{1}-\omega_{2}\right)}+\frac{\left(1-\omega_{2}\right)}{\left(1-\omega_{1}-\omega_{2}\right)} D_{11}
\end{array}\right\}\left(\frac{\partial^{2} \omega_{1}}{\partial z^{2}}\right)+\left\{D_{22} \frac{\omega_{1}}{\left(1-\omega_{1}-\omega_{2}\right)}+D_{21} \frac{\left(1-\omega_{2}\right)}{\left(1-\omega_{1}-\omega_{2}\right)}\right\} \\
& \left(\frac{\partial^{2} \omega_{2}}{\partial z^{2}}\right)+
\end{aligned}
$$




$$
\begin{aligned}
& \left\{\begin{array}{l}
\frac{\omega_{1}}{\left(1-\omega_{1}-\omega_{2}\right)}\left(\frac{\partial D_{22}}{\partial \omega_{1}}\right)+\frac{\omega_{1}}{\left(1-\omega_{1}-\omega_{2}\right)}\left(\frac{\partial D_{21}}{\partial \omega_{2}}\right)+\frac{\left(1-\omega_{2}\right)}{\left(1-\omega_{1}-\omega_{2}\right)}\left(\frac{\partial D_{11}}{\partial \omega_{2}}\right)+ \\
\frac{\left(1-\omega_{2}\right)}{\left(1-\omega_{1}-\omega_{2}\right)} \\
\left(\frac{\partial D_{12}}{\partial \omega_{1}}\right)
\end{array}\right\}\left(\frac{\partial \omega_{1}}{\partial z} \frac{\partial \omega_{2}}{\partial z}\right) \\
& +\left\{\frac{\omega_{1}}{\left(1-\omega_{1}-\omega_{2}\right)}\left(\frac{\partial D_{21}}{\partial \omega_{1}}\right)+\frac{\left(1-\omega_{2}\right)}{\left(1-\omega_{1}-\omega_{2}\right)}\left(\frac{\partial D_{11}}{\partial \omega_{1}}\right)\right\}\left(\frac{\partial \omega_{1}}{\partial z}\right)^{2} \\
& +\left\{\frac{1-\omega_{2}}{\left(1-\omega_{1}-\omega_{2}\right)}\left(\frac{\partial D_{12}}{\partial \omega_{2}}\right)+\frac{\omega_{1}}{\left(1-\omega_{1}-\omega_{2}\right)}\left(\frac{\partial D_{22}}{\partial \omega_{2}}\right)\right\}\left(\frac{\partial \omega_{2}}{\partial z}\right)^{2}
\end{aligned}
$$

Equation (4.17) is the mass transfer model for gas 1 . Theoretical mass transfer model for second gas can be obtained by interchanging the subscript 1 and 2 in Equation (4.17). The mathematical model for second gas is given by Equation (4.18).

$$
\begin{aligned}
& f_{2}=\frac{\partial \omega_{2}}{\partial t}=\left\{\begin{array}{l}
\left(\frac{\omega_{2}}{\left(1-\omega_{1}-\omega_{2}\right)^{2}}+\frac{1}{1-\omega_{1}-\omega_{2}}\right)\left(D_{11} \frac{\partial \omega_{1}}{\partial z}+D_{12} \frac{\partial \omega_{2}}{\partial z}\right)+\left(\frac{1-\omega_{1}}{\left(1-\omega_{1}-\omega_{2}\right)^{2}}\right) \\
\left(D_{22} \frac{\partial \omega_{2}}{\partial z}+D_{21} \frac{\partial \omega_{1}}{\partial z}\right)
\end{array}\right\} \frac{\partial \omega_{2}}{\partial z}+ \\
& \left\{\begin{array}{l}
\left(\frac{\omega_{2}}{\left(1-\omega_{1}-\omega_{2}\right)^{2}}\right)\left(D_{11} \frac{\partial \omega_{1}}{\partial z}+D_{12} \frac{\partial \omega_{2}}{\partial z}\right)+\left(\frac{1-\omega_{1}}{\left(1-\omega_{1}-\omega_{2}\right)^{2}}-\frac{1}{1-\omega_{1}-\omega_{2}}\right) \\
\left(D_{22} \frac{\partial \omega_{2}}{\partial z}+D_{21} \frac{\partial \omega_{1}}{\partial z}\right)
\end{array}\right\} \frac{\partial \omega_{1}}{\partial z}+ \\
& \left\{\mathrm{D}_{12} \frac{\omega_{2}}{\left(1-\omega_{1}-\omega_{2}\right)}+\frac{\left(1-\omega_{1}\right)}{\left(1-\omega_{1}-\omega_{2}\right)} \mathrm{D}_{22}\right\}\left(\frac{\partial^{2} \omega_{2}}{\partial \mathrm{z}^{2}}\right)+\left\{\mathrm{D}_{11} \frac{\omega_{2}}{\left(1-\omega_{1}-\omega_{2}\right)}+\mathrm{D}_{12} \frac{\left(1-\omega_{1}\right)}{\left(1-\omega_{1}-\omega_{2}\right)}\right\} \\
& \left(\frac{\partial^{2} \omega_{1}}{\partial \mathbf{z}^{2}}\right)+
\end{aligned}
$$




$$
\begin{aligned}
& \left\{\begin{array}{l}
\left.\frac{\omega_{2}}{\left(1-\omega_{1}-\omega_{2}\right)}\left(\frac{\partial \mathrm{D}_{11}}{\partial \omega_{2}}\right)+\frac{\omega_{2}}{\left(1-\omega_{1}-\omega_{2}\right)}\left(\frac{\partial \mathrm{D}_{12}}{\partial \omega_{1}}\right)+\frac{\left(1-\omega_{1}\right)}{\left(1-\omega_{1}-\omega_{2}\right)}\left(\frac{\partial \mathrm{D}_{22}}{\partial \omega_{1}}\right)+\right\}\left(\frac{\partial \omega_{1}}{\partial \mathrm{z}} \frac{\partial \omega_{2}}{\partial \mathrm{z}}\right) \\
\frac{\left(1-\omega_{1}\right)}{\left(1-\omega_{1}-\omega_{2}\right)}\left(\frac{\partial \mathrm{D}_{21}}{\partial \omega_{2}}\right)
\end{array}\right. \\
& +\left\{\frac{\omega_{2}}{\left(1-\omega_{1}-\omega_{2}\right)}\left(\frac{\partial \mathrm{D}_{12}}{\partial \omega_{2}}\right)+\frac{\left(1-\omega_{1}\right)}{\left(1-\omega_{1}-\omega_{2}\right)}\left(\frac{\partial \mathrm{D}_{22}}{\partial \omega_{2}}\right)\right\}\left(\frac{\partial \omega_{2}}{\partial \mathrm{z}}\right)^{2} \\
& +\left\{\frac{1-\omega_{1}}{\left(1-\omega_{1}-\omega_{2}\right)}\left(\frac{\partial \mathrm{D}_{21}}{\partial \omega_{1}}\right)+\frac{\omega_{2}}{\left(1-\omega_{1}-\omega_{2}\right)}\left(\frac{\partial \mathrm{D}_{11}}{\partial \omega_{1}}\right)\right\}\left(\frac{\partial \omega_{1}}{\partial \mathrm{z}}\right)^{2}
\end{aligned}
$$

In Equations (4.17) and Equation (4.18) $D_{11}, D_{12,}, D_{21}$, and $D_{22}$, are the concentrationdependent, multi-component diffusivities of two gases in a non-volatile, dense phase.

Equations (4.17) and (4.18) have the following initial conditions:

$$
\begin{array}{ll}
\omega_{i}(0,0)=\omega_{e q, i}(t=0) & \text { at the gas-liquid interface } \\
\omega_{i}(\mathrm{z}, 0)=0 & \text { for } 0 \leq z \leq L
\end{array}
$$

and the boundary conditions:

$$
\begin{array}{ll}
\omega_{i}(0, t)=\omega_{e q}(t) & \text { for } 0<t \leq t_{f} \\
\left.\frac{\partial \omega_{i}}{\partial z}\right|_{z=L}=0 & \text { for } 0<t \leq t_{f}
\end{array}
$$

where $i=1,2$ and $\omega_{e q, i}$ is the equilibrium saturation concentration of the $\mathrm{i}^{\text {th }}$ gas at the interface.

To determine the concentration-dependent, multicomponent diffusivities using our mass transfer model, we conducted two kinds of experiments, i.e., solubility experiments and 
diffusion experiments. The purpose of the solubility experiments was to determine the equilibrium concentration of each gas at the gas-polymer interface as a function of pressure and gas-phase composition. This provided the boundary conditions. The purpose of the diffusion experiments was to obtain data for pressure and the gas-phase composition as a function of time. These data provided the experimental mass of each gas absorbed in the dense phase.

To solve our mass transfer model, the four unknown diffusivities, $\mathrm{D}_{11}, \mathrm{D}_{12}, \mathrm{D}_{21}$, and $\mathrm{D}_{22}$, are required. This is an inverse problem that can be solved using the optimal control technique.

\subsection{Optimal Control}

An optimal control involves the improvement in the system's performance as a function of time, space, or any other independent variable. In our study, the optimal control technique was used to extract the optimal, composition-dependent, multi-component diffusivities (system property) as a function of another system property (composition). Unknown diffusivities are control surfaces that must be optimally determined such that the difference between the experimental and calculated masses is minimized. The error function is the objective function that is described next (Upreti, 2013). 


\subsection{The Objective Functional}

The goal is to determine the unknown, concentration-dependent, multi-component diffusivities of the two gases in the non-volatile dense phase such that their use in equations (4.19) to (4.22) yields the calculated masses of the gases in the layer that are in agreement with experimentally-determined masses of the gases, i.e., $m_{1 \exp }(t)$ and $m_{2 \exp }(t)$ through gas chromatograph and pressure decay measurements. The objective functional can be expressed as the summation of:

$I_{1}=\int_{0}^{T}\left[m_{1 \mathrm{mod}}(t)-m_{1 \exp }(t)\right]^{2} d t$

and

$I_{2}=\int_{0}^{T}\left[m_{2 \bmod }(t)-m_{2 \exp }(t)\right]^{2} d t$

where $I_{1}$ and $I_{2}$ are the measures of error between the predicted and experimental gas masses for the respective gases over time $T$.

At any given time, $m_{1 \mathrm{mod}}(t)$ and $m_{1 \mathrm{mod}}(t)$ are the model-predicted mass of gases absorbed in the polymer, whereas, $\mathrm{m}_{1 \exp }(\mathrm{t})$ and $\mathrm{m}_{2 \exp }(\mathrm{t})$ are the experimental-determined gas masses absorbed in the polymer. The model-predicted mass of each gas absorbed is given by:

$m_{1 \mathrm{mod}}=\int_{0}^{L} \rho \omega_{1} A d z$ and, $m_{2 \mathrm{mod}}=\int_{0}^{L} \rho \omega_{2} A d z$ 
Thus,

$$
I=I_{1}+I_{2}=\int_{0}^{T} \int_{0}^{L}\left[\left(\omega_{1} \rho A d z-m_{1 \exp }\right)^{2}+\left(\omega_{2} \rho A d z-m_{2 \exp }\right)^{2}\right] d t
$$

where $L$ is the depth of the polymer phase having a cross-sectional area $A$. Note that $\omega_{i}(z, t)$ is given by the highly non-linear, partial differential Equations (4.17) and (4.18), having diffusivities $D_{11}\left(\omega_{1}, \omega_{2}\right), D_{12}\left(\omega_{1}, \omega_{2}\right), D_{21}\left(\omega_{1}, \omega_{2}\right), D_{22}\left(\omega_{1}, \omega_{2}\right)$ as the twodimensional surfaces to be optimally determined.

The optimal control problem is to determine these functions that minimizes I given by Equation (4.26) subject to the satisfaction of differential equation constraints. So, Equation (4.17) can also be written as:

$$
\int G_{1}(z, t)=\frac{\partial \omega_{1}}{\partial t}-f_{1}\left(\omega_{1}, \omega_{2}, \frac{\partial \omega_{1}}{\partial z}, \frac{\partial \omega_{2}}{\partial z}, \frac{\partial^{2} \omega_{1}}{\partial z^{2}}, \frac{\partial^{2} \omega_{2}}{\partial z^{2}}, D_{11}, D_{12}, D_{21}, D_{22}\right)=0
$$

For the second gas, Equation (4.18) can be written as:

$$
G_{2}(z, t)=\frac{\partial \omega_{2}}{\partial t}-f_{2}\left(\omega_{1}, \omega_{2}, \frac{\partial \omega_{1}}{\partial z}, \frac{\partial \omega_{2}}{\partial z}, \frac{\partial^{2} \omega_{1}}{\partial z^{2}}, \frac{\partial^{2} \omega_{2}}{\partial z^{2}}, D_{11}, D_{12}, D_{21}, D_{22}\right)=0
$$

The constrained problem above is equivalent to the unconstrained minimization of:

$$
J=I_{1}+I_{2}+\int_{0}^{T} \int_{0}^{L}\left(\lambda_{1} G_{1}+\lambda_{2} G_{2}\right) d z d t
$$

where $J$ is the augmented objective functional and $\lambda_{i}(z, t)$ is a costate variable 
In the next section, we derive necessary condition for $\mathrm{J}$ to be at its minimum value. The solution of necessary conditions will yield the unknown concentration dependent multicomponent diffusivities.

\subsection{Necessary Condition for the Minimum}

The necessary condition for the minimum is that the variation of $J$ is zero, i.e.:

$\delta J=\delta I_{1}+\delta I_{2}+\int_{0}^{T} \int_{0}^{L}\left(\delta \delta_{1} G_{1}+\lambda_{1} \delta G_{1}+\delta \lambda_{2} G_{2}+\lambda_{2} \delta G_{2}\right) d z d t=0$

In the above equation:

$\delta I_{1}=\int_{0}^{T} \int_{0}^{L} 2 A \rho\left(m_{1 \bmod }-m_{1 \exp }\right) \delta \omega_{1} d z d t$

$\delta I_{2}=\int_{0}^{T} \int_{0}^{L} 2 A \rho\left(m_{2 \bmod }-m_{2 \exp }\right) \delta \omega_{2} d z d t$

$\delta G_{1}$ and $\delta G_{2}$, respectively, are given by:

$$
\begin{aligned}
& \delta G_{1}=\frac{\partial}{\partial t} \delta \omega_{1}-\frac{\partial f_{1}}{\partial \omega_{1}} \delta \omega_{1}-\frac{\partial f_{1}}{\partial \omega_{2}} \delta \omega_{2}-\frac{\partial f_{1}}{\partial \omega_{1 Z}} \delta \omega_{1 Z}-\frac{\partial f_{1}}{\partial \omega_{2 Z}} \delta \omega_{2 Z}-\frac{\partial f_{1}}{\partial \omega_{1 Z}} \delta \omega_{1 Z}-\frac{\partial f_{1}}{\partial \omega_{2 Z}} \delta \omega_{2 Z}-\frac{\partial f_{1}}{\partial D_{11}} \delta D_{11} \\
& -\frac{\partial f_{1}}{\partial D_{12}} \delta D_{12}-\frac{\partial f_{1}}{\partial D_{21}} \delta D_{21}-\frac{\partial f_{1}}{\partial D_{22}} \delta D_{22}
\end{aligned}
$$




$$
\begin{aligned}
& \delta G_{2}=\frac{\partial}{\partial t} \delta \omega_{2}-\frac{\partial f_{2}}{\partial \omega_{2}} \delta \omega_{2}-\frac{\partial f_{2}}{\partial \omega_{1}} \delta \omega_{1}-\frac{\partial f_{2}}{\partial \omega_{2 Z}} \delta \omega_{2 Z}-\frac{\partial f_{2}}{\partial \omega_{1 Z}} \delta \omega_{1 Z}-\frac{\partial f_{2}}{\partial \omega_{2 Z}} \delta \omega_{2 Z Z}-\frac{\partial f_{2}}{\partial \omega_{1 Z Z}} \delta \omega_{1 Z}-\frac{\partial f_{2}}{\partial D_{11}} \delta D_{11} \\
& -\frac{\partial f_{2}}{\partial D_{12}} \delta D_{12}-\frac{\partial f_{2}}{\partial D_{21}} \delta D_{21}-\frac{\partial f_{2}}{\partial D_{22}} \delta D_{22}
\end{aligned}
$$

Substituting Equations (4.31-4.34) into Equation (4.30) we get:

$$
\begin{aligned}
& \delta J=\int_{0}^{T} \int_{0}^{L}[\underbrace{2 A \rho\left(m_{\mathrm{mod}}-m_{\mathrm{exp}}\right)_{1} \delta \omega_{1}}_{\text {Gas } 1}+\underbrace{2 A \rho\left(m_{\mathrm{mod}}-m_{\mathrm{exp}}\right)_{2} \delta \omega_{2}}_{\text {Gas } 2}] d z d t+ \\
& \int_{0}^{T} \int_{0}^{L} \lambda_{1}\left(\frac{\partial}{\partial t} \delta \omega_{1}-\frac{\partial f_{1}}{\partial \omega_{1}} \delta \omega_{1}-\frac{\partial f_{1}}{\partial \omega_{2}} \delta \omega_{2}-\frac{\partial f_{1}}{\partial \omega_{1 Z}} \delta \omega_{1 Z}-\frac{\partial f_{1}}{\partial \omega_{2 Z}} \delta \omega_{2 Z}-\frac{\partial f_{1}}{\partial \omega_{1 Z Z}} \delta \omega_{1 Z Z}\right. \\
& \underbrace{\left.-\frac{\partial f_{1}}{\partial \omega_{2 Z Z}} \delta \omega_{2 Z Z}-\frac{\partial f_{1}}{\partial D_{11}} \delta D_{11}-\frac{\partial f_{1}}{\partial D_{12}} \delta D_{12}-\frac{\partial f_{1}}{\partial D_{21}} \delta D_{21}-\frac{\partial f_{1}}{\partial D_{22}} \delta D_{22}\right) d z d t}_{\text {Gas } 1} \\
& \int_{0}^{T} \int_{0}^{L}\left(\lambda_{2} \frac{\partial}{\partial t} \delta \omega_{2}-\frac{\partial f_{2}}{\partial \omega_{2}} \delta \omega_{2}-\frac{\partial f_{2}}{\partial \omega_{1}} \delta \omega_{1}-\frac{\partial f_{2}}{\partial \omega_{2 Z}} \delta \omega_{2 Z}-\frac{\partial f_{2}}{\partial \omega_{1 Z}} \delta \omega_{1 Z}-\frac{\partial f_{2}}{\partial \omega_{2 Z Z}} \delta \omega_{2 Z Z}-\right. \\
& +\frac{\partial f_{2}}{\partial \omega_{1 Z Z}} \delta \omega_{1 Z Z} \\
& \underbrace{\left.\frac{\partial f_{2}}{\partial D_{11}} \delta D_{11}-\frac{\partial f_{2}}{\partial D_{12}} \delta D_{12}-\frac{\partial f_{2}}{\partial D_{21}} \delta D_{21}-\frac{\partial f_{2}}{\partial D_{22}} \delta D_{22}\right) d z d t}_{\text {Gas } 2}
\end{aligned}
$$

variation $\delta J$ is a combination of $\delta J_{1}$ (for gas 1 ) and $\delta J_{2}$ (for gas 2).

$\delta J=\delta J_{1}+\delta J_{2}$

where $\delta J_{1}$ and $\delta J_{2}$ are given by 


$$
\begin{aligned}
& \delta J_{1}=\int_{0}^{T} \int_{0}^{L}\left[2 A \rho\left(m_{\mathrm{mod}}-m_{\mathrm{exp}}\right)_{1} \delta \omega_{1}\right] d z d t+\int_{0}^{T} \int_{0}^{L} \lambda_{1}\left(\frac{\partial}{\partial t} \delta \omega_{1}-\frac{\partial f_{1}}{\partial \omega_{1}} \delta \omega_{1}-\frac{\partial f_{1}}{\partial \omega_{2}} \delta \omega_{2}-\frac{\partial f_{1}}{\partial \omega_{1 Z}} \delta \omega_{1 Z}\right. \\
& -\frac{\partial f_{1}}{\partial \omega_{2 Z}} \delta \omega_{2 Z}-\frac{\partial f_{1}}{\partial \omega_{1 Z}} \delta \omega_{1 Z}-\frac{\partial f_{1}}{\partial \omega_{2 Z}} \delta \omega_{2 Z}-\frac{\partial f_{1}}{\partial D_{11}} \delta D_{11}-\frac{\partial f_{1}}{\partial D_{12}} \delta D_{12}-\frac{\partial f_{1}}{\partial D_{21}} \delta D_{21}- \\
& \left.\frac{\partial f_{1}}{\partial D_{22}} \delta D_{22}\right) d z d t
\end{aligned}
$$

and

$$
\begin{aligned}
& \delta J_{2}=\int_{0}^{T} \int_{0}^{L}\left[2 A \rho\left(m_{\text {mod }}-m_{\text {exp }}\right)_{2} \delta \omega_{2}\right] d z d t+\int_{0}^{T} \int_{0}^{L}\left(\lambda_{2} \frac{\partial}{\partial t} \delta \omega_{2}-\frac{\partial f_{2}}{\partial \omega_{2}} \delta \omega_{2}-\frac{\partial f_{2}}{\partial \omega_{1}} \delta \omega_{1}-\frac{\partial f_{2}}{\partial \omega_{2 Z}} \delta \omega_{2 Z}\right. \\
& -\frac{\partial f_{2}}{\partial \omega_{1 Z}} \delta \omega_{1 Z}-\frac{\partial f_{2}}{\partial \omega_{2 Z Z}} \delta \omega_{2 Z Z}-\frac{\partial f_{2}}{\partial \omega_{1 Z Z}} \delta \omega_{1 Z Z}-\frac{\partial f_{2}}{\partial D_{11}} \delta D_{11}-\frac{\partial f_{2}}{\partial D_{12}} \delta D_{12}-\frac{\partial f_{2}}{\partial D_{21}} \delta D_{21}- \\
& \left.\frac{\partial f_{2}}{\partial D_{22}} \delta D_{22}\right) d z d t
\end{aligned}
$$

For the sake of simplicity we first simplify the terms on the R.H.S of Equation (4.36) for gas $1:$

Integrating by parts the third, sixth, seventh, eighth, and ninth terms in the Equation (4.36), we get:

$$
\begin{aligned}
& \int_{0}^{T} \int_{0}^{L}\left(\lambda_{1} \frac{\partial}{\partial t} \delta \omega_{1}\right) d z d t=\int_{0}^{L}\left[\lambda_{1} \frac{\partial}{\partial t} \delta \omega_{1} d z\right]_{0}^{T}-\int_{0}^{T} \int_{0}^{L} \dot{\lambda}_{1} \delta \omega_{1} d z d t \\
& -\int_{0}^{T} \int_{0}^{L}\left(\lambda_{1} \frac{\partial f_{1}}{\partial \omega_{1 Z}} \delta \omega_{1 Z}\right) d z d t=-\int_{0}^{T}\left[\lambda_{1} \frac{\partial f_{1}}{\partial \omega_{1 Z}} \delta \omega_{1}\right]_{0}^{L} d t+\int_{0}^{T} \int_{0}^{L} \frac{\partial}{\partial z}\left(\lambda_{1} \frac{\partial f_{1}}{\partial \omega_{1 Z}}\right) \delta \omega_{1} d z d t \\
& -\int_{0}^{T} \int_{0}^{L}\left(\lambda_{1} \frac{\partial f_{1}}{\partial \omega_{2 Z}} \delta \omega_{2 Z}\right) d z d t=-\int_{0}^{T}\left[\lambda_{1} \frac{\partial f_{1}}{\partial \omega_{2 Z}} \delta \omega_{2}\right]_{0}^{L} d t+\int_{0}^{T} \int_{0}^{L} \frac{\partial}{\partial z}\left(\lambda_{1} \frac{\partial f_{1}}{\partial \omega_{2 Z}}\right) \delta \omega_{2} d z d t
\end{aligned}
$$




$$
\begin{aligned}
& \left.-\int_{00}^{T L} \int_{1} \frac{\partial f_{1}}{\partial \omega_{1 Z}} \delta \omega_{1 Z}\right) d z d t=-\int_{0}^{T}\left[\lambda_{1} \frac{\partial f_{1}}{\partial \omega_{1 Z}} \delta \omega_{1 Z}\right]_{0}^{L} d t+\int_{0}^{T}\left[\frac{\partial}{\partial z}\left(\lambda_{1} \frac{\partial f_{1}}{\partial \omega_{1 Z}}\right) \delta \omega_{1}\right]_{0}^{L} d t-\int_{00}^{T L} \int_{0}^{T} \frac{\partial^{2}}{\partial z^{2}}\left(\lambda_{1} \frac{\partial f_{1}}{\partial \omega_{1 Z}}\right) \delta \omega_{1} d z d t \\
& -\int_{0}^{T} \int_{0}^{L}\left(\lambda_{1} \frac{\partial f_{1}}{\partial \omega_{2 Z}} \delta \omega_{2 Z}\right) d z d t=-\int_{0}^{T}\left[\lambda_{1} \frac{\partial f_{1}}{\partial \omega_{2 Z}} \delta \omega_{2 Z}\right]_{0}^{L} d t+\int_{0}^{T}\left[\frac{\partial}{\partial z}\left(\lambda_{1} \frac{\partial f_{1}}{\partial \omega_{2 Z}}\right) \delta \omega_{2}\right]_{0}^{L} d t-\int_{0}^{T} \int_{0}^{L} \frac{\partial^{2}}{\partial z^{2}}\left(\lambda_{1} \frac{\partial f_{1}}{\partial \omega_{2 Z}}\right) \delta \omega_{2} d z d t
\end{aligned}
$$

Substituting Equations (4.38-3.42) into Equation (4.36) and rearranging gives:

$$
\begin{aligned}
& \delta J_{1}=\int_{0}^{T} \int_{0}^{L}\left(\delta I_{1}-\frac{\partial}{\partial t} \lambda_{1} d z d t-\lambda_{1} \frac{\partial f_{1}}{\partial \omega_{11}}+\frac{\partial}{\partial z}\left(\lambda_{1} \frac{\partial f_{1}}{\partial \omega_{1 Z}}\right)-\frac{\partial^{2}}{\partial z^{2}}\left(\lambda_{1} \frac{\partial f_{1}}{\partial \omega_{1 Z Z}}\right)\right) \delta \omega_{1} d z \\
& -\int_{0}^{T} \int_{0}^{L}\left(\lambda_{1} \frac{\partial f_{1}}{\partial \omega_{2}}-\frac{\partial}{\partial z}\left(\lambda_{1} \frac{\partial f_{1}}{\partial \omega_{2 Z}}\right)+\frac{\partial^{2}}{\partial z^{2}}\left(\lambda_{1} \frac{\partial f_{1}}{\partial \omega_{2 Z}}\right)\right) \delta \omega_{2} d z d t \\
& -\int_{0}^{T}\left[\lambda_{1} \frac{\partial f_{1}}{\partial \omega_{1 z}}-\frac{\partial}{\partial z}\left(\lambda_{1} \frac{\partial f_{1}}{\partial \omega_{1 Z}}\right)\right]_{0}^{L} \delta \omega_{1} d t \\
& +\int_{0}^{L}\left[\lambda_{1} \delta \omega_{1}\right]_{0}^{T} d z \\
& -\int_{0}^{T}\left[\lambda_{1} \frac{\partial f_{1}}{\partial \omega_{2 Z}}-\frac{\partial}{\partial z}\left(\lambda_{1} \frac{\partial f_{1}}{\partial \omega_{2 Z}}\right)\right]_{0}^{L} \delta \omega_{2} d t \\
& -\int_{0}^{T}\left[\lambda_{1} \frac{\partial f_{1}}{\partial \omega_{1 Z}}\right]_{0}^{L} \delta \omega_{1 z} d t-\int_{0}^{T}\left[\lambda_{1} \frac{\partial f_{1}}{\partial \omega_{2 Z}}\right]_{0}^{L} \delta \omega_{2 z} d t \\
& \left.-\int_{0}^{T} \int_{0}^{L}\left(\lambda_{1} \frac{\partial f_{1}}{\partial D_{11}} \delta D_{11}+\lambda_{1} \frac{\partial f_{1}}{\partial D_{12}} \delta D_{12}+\lambda_{1} \frac{\partial f_{1}}{\partial D_{21}} \delta D_{21}+\lambda_{1} \frac{\partial f_{1}}{\partial D_{22}} \delta D_{22}\right)\right) d z d t
\end{aligned}
$$

Similarly, $\delta J_{2}$ can be derived by interchanging subscripts 1 and 2 in Equation (4.43).

Because the initial mass concentration of the gas in the polymer is known at the interface and is zero everywhere in the polymer, the variation $\delta \omega_{1}(z, 0)$ and $\delta \omega_{2}(z, 0)$ is zero for all 
values of $z$. Since the final mass concentration of the gas is not specified, the fourth integral in Equation (4.43) is eliminated by forcing:

$\lambda_{i}(z, T)=0 \quad 0 \leq z \leq L \quad i=1,2$

Since the equilibrium concentration of gas at the interface $\left[\omega_{1}(0, t)=\omega_{1 \text { sat }}(t)\right]$ and $\left[\omega_{2}(0, t)=\omega_{2 \text { sat }}(t)\right]$ is always specified, $\delta \omega_{10 r 2}(0, t)$ is zero. Therefore, the third and fifth integrals are eliminated in Equation (4.43). Furthermore, by forcing:

$$
\begin{array}{lll}
\lambda_{i}(L, t)=0 & 0 \leq t \leq T & i=1,2 \\
\lambda_{i}(0, t)=0 & 0 \leq t \leq T & i=1,2
\end{array}
$$

the sixth terms in equation (4.43) are eliminated. Similarly variation of gas $2 \delta J_{2}$ can also be simplified. Simplifying and rearranging Equation (4.35), we get: 


$$
\begin{aligned}
& \delta J=\left\{\begin{array}{l}
{\left[\int_{0}^{T} \int_{0}^{L}\left(2 A \rho\left(m_{\bmod }-m_{\exp }\right)_{1}-\frac{\partial}{\partial t} \lambda_{1}-\lambda_{1} \frac{\partial f_{1}}{\partial \omega_{1}}+\frac{\partial}{\partial z}\left(\lambda_{1} \frac{\partial f_{1}}{\partial \omega_{1 Z}}\right)-\frac{\partial^{2}}{\partial z^{2}}\left(\lambda_{1} \frac{\partial f_{1}}{\partial \omega_{1 Z Z}}\right)\right)\right]} \\
+\left[\int_{0}^{T} \int_{0}^{L}\left(\lambda_{2} \frac{\partial f_{2}}{\partial \omega_{1}}-\frac{\partial}{\partial z}\left(\lambda_{2} \frac{\partial f_{2}}{\partial \omega_{1 Z}}\right)+\frac{\partial^{2}}{\partial z^{2}}\left(\lambda_{2} \frac{\partial f_{2}}{\partial \omega_{1 Z Z}}\right)\right)\right]
\end{array}\right\} \delta \omega_{1} d z d t
\end{aligned}
$$

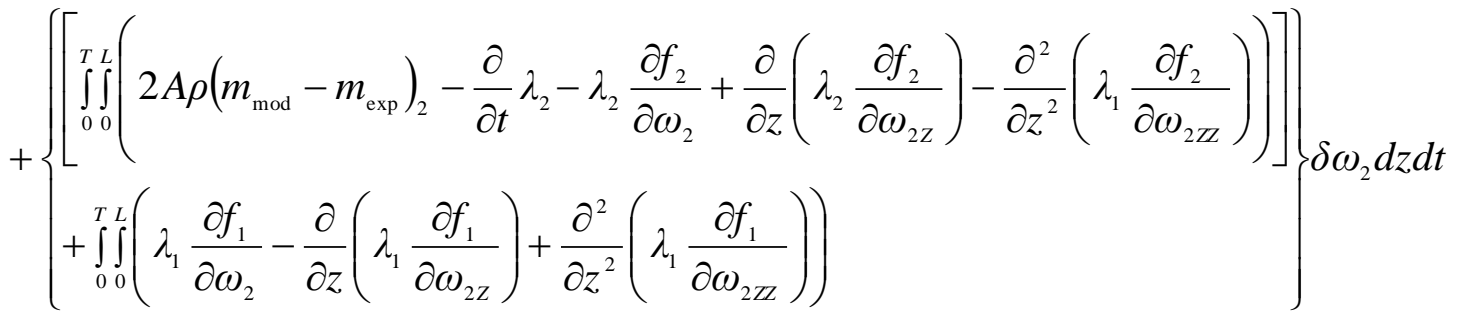

$$
\begin{aligned}
& -\int_{0}^{T} \int_{0}^{L}\left(\lambda_{1} \frac{\partial f_{1}}{\partial D_{11}} \delta D_{11}+\lambda_{1} \frac{\partial f_{1}}{\partial D_{12}} \delta D_{12}+\lambda_{1} \frac{\partial f_{1}}{\partial D_{21}} \delta D_{21}+\lambda_{1} \frac{\partial f_{1}}{\partial D_{22}} \delta D_{22}\right) d z d t \\
& -\int_{0}^{T} \int_{0}^{L}\left(\lambda_{2} \frac{\partial f_{2}}{\partial D_{11}} \delta D_{11}+\lambda_{2} \frac{\partial f_{2}}{\partial D_{12}} \delta D_{12}+\lambda_{2} \frac{\partial f_{2}}{\partial D_{21}} \delta D_{21}+\lambda_{2} \frac{\partial f_{2}}{\partial D_{22}} \delta D_{22}\right) d z d t
\end{aligned}
$$

In the above equation, the first integral is eliminated by defining $\frac{\partial \lambda_{1}}{\partial t}$ as follows:

$$
\begin{aligned}
& \frac{\partial \lambda_{1}}{\partial t}=2 A \rho\left(m_{\text {mod }}-m_{\text {exp }}\right)_{1}-\lambda_{1} \frac{\partial f_{1}}{\partial \omega_{1}}+\frac{\partial}{\partial z}\left(\lambda_{1} \frac{\partial f_{1}}{\partial \omega_{1 Z}}\right)-\frac{\partial^{2}}{\partial z^{2}}\left(\lambda_{1} \frac{\partial f_{1}}{\partial \omega_{1 Z Z}}\right)+ \\
& \lambda_{2} \frac{\partial f_{2}}{\partial \omega_{1}}-\frac{\partial}{\partial z}\left(\lambda_{2} \frac{\partial f_{2}}{\partial \omega_{1 Z}}\right)+\frac{\partial^{2}}{\partial z^{2}}\left(\lambda_{2} \frac{\partial f_{2}}{\partial \omega_{1 Z Z}}\right)
\end{aligned}
$$

and second integral in Equation (4.47) can be eliminated by defining $\frac{\partial \lambda_{2}}{\partial t}$ as follows:

$$
\begin{aligned}
& \frac{\partial \lambda_{2}}{\partial t}=\left(2 A \rho\left(m_{\text {mod }}-m_{\text {exp }}\right)_{2}-\lambda_{2} \frac{\partial f_{2}}{\partial \omega_{2}}+\frac{\partial}{\partial z}\left(\lambda_{2} \frac{\partial f_{2}}{\partial \omega_{2 Z}}\right)-\frac{\partial^{2}}{\partial z^{2}}\left(\lambda_{1} \frac{\partial f_{2}}{\partial \omega_{2 Z Z}}\right)\right)+ \\
& \lambda_{1} \frac{\partial f_{1}}{\partial \omega_{2}}-\frac{\partial}{\partial z}\left(\lambda_{1} \frac{\partial f_{1}}{\partial \omega_{2 Z}}\right)+\frac{\partial^{2}}{\partial z^{2}}\left(\lambda_{1} \frac{\partial f_{1}}{\partial \omega_{2 Z z}}\right)
\end{aligned}
$$


Equation (4.44) is the initial condition for Equations (4.48) and (4.49), and Equations (4.45) and (4.46) are its two boundary conditions. Equations (4.48) and (4.49) are also called costate equations.

Hence, subject to Equations (4.48) and (4.49), Equation (4.47) can be simplified to:

$\delta J=-\int_{0}^{T} \int_{0}^{L}\left(\begin{array}{l}\lambda_{1} \frac{\partial f_{1}}{\partial D_{11}} \delta D_{11}+\lambda_{1} \frac{\partial f_{1}}{\partial D_{12}} \delta D_{12}+\lambda_{1} \frac{\partial f_{1}}{\partial D_{21}} \delta D_{21}+\lambda_{1} \frac{\partial f_{1}}{\partial D_{22}} \delta D_{22}+ \\ \lambda_{2} \frac{\partial f_{2}}{\partial D_{11}} \delta D_{11}+\lambda_{2} \frac{\partial f_{2}}{\partial D_{12}} \delta D_{12}+\lambda_{2} \frac{\partial f_{2}}{\partial D_{21}} \delta D_{21}+\lambda_{2} \frac{\partial f_{2}}{\partial D_{22}} \delta D_{22}\end{array}\right) d z d t=0$

since $\delta D_{i j}$, where $i, j=1,2$ are arbitrary, their coefficients must be individually equal to zero.

Thus, at the minimum of $J$, the variational derivative of $J$ with respect to , $D_{11}, D_{12,} D_{21}$, and $D_{22}$ is zero, i.e.,:

$$
\begin{aligned}
& -\left(\lambda_{1} \frac{\partial f_{1}}{\partial D_{11}}+\lambda_{2} \frac{\partial f_{2}}{\partial D_{11}}\right)=0 \\
& -\left(\lambda_{1} \frac{\partial f_{1}}{\partial D_{12}}+\lambda_{2} \frac{\partial f_{2}}{\partial D_{12}}\right)=0 \\
& -\left(\lambda_{1} \frac{\partial f_{1}}{\partial D_{21}}+\lambda_{2} \frac{\partial f_{2}}{\partial D_{21}}\right)=0 \\
& -\left(\lambda_{1} \frac{\partial f_{1}}{\partial D_{22}}+\lambda_{2} \frac{\partial f_{2}}{\partial D_{22}}\right)=0
\end{aligned}
$$


The negative of the variational derivative provides the gradient correction for $D_{i j}\left(\omega_{1}, \omega_{2}\right)$ in the iterative minimization of $J$. To summarize, Equations (4.44-4.46), (4.48-4.49), and (4.51-4.54) form the set of necessary conditions.

The detailed derivation for the derivatives of the terms $\frac{\partial f_{1}}{\partial \omega_{1}}, \frac{\partial}{\partial z}\left(\frac{\partial f_{1}}{\partial \omega_{1 z}}\right), \frac{\partial^{2}}{\partial z^{2}}\left(\frac{\partial f_{1}}{\partial \omega_{1 Z Z}}\right)$ and $\frac{\partial \mathrm{f}_{1}}{\partial \omega_{2}}, \frac{\partial}{\partial \mathbf{z}}\left(\frac{\partial \mathrm{f}_{1}}{\partial \omega_{2 \mathrm{z}}}\right), \frac{\partial^{2}}{\partial \mathbf{z}^{2}}\left(\frac{\partial \mathrm{f}_{1}}{\partial \omega_{2 \mathrm{zz}}}\right)$ are given below.

Differentiating $f_{1}$ w.r.t $\omega_{1}$ we get

$$
\begin{aligned}
& \frac{\partial f_{1}}{\partial \omega_{1}}=\left\{\begin{array}{l}
\left(\frac{2 \omega_{1}}{\left(1-\omega_{1}-\omega_{2}\right)^{3}}+\frac{1}{\left(1-\omega_{1}-\omega_{2}\right)^{2}}+\frac{1}{\left(1-\omega_{1}-\omega_{2}\right)^{2}}\right)\left(D_{22} \frac{\partial \omega_{2}}{\partial z}+D_{21} \frac{\partial \omega_{1}}{\partial z}\right)+\left(\frac{2\left(1-\omega_{2}\right)}{\left(1-\omega_{1}-\omega_{2}\right)^{3}}\right)\left(D_{11} \frac{\partial \omega_{1}}{\partial z}+D_{12} \frac{\partial \omega_{2}}{\partial z}\right)+ \\
\left(\frac{\omega_{1}}{\left(1-\omega_{1}-\omega_{2}\right)^{2}}+\frac{1}{1-\omega_{1}-\omega_{2}}\right)\left(\frac{\partial D_{22}}{\partial \omega_{1}} \frac{\partial \omega_{2}}{\partial z}+\frac{\partial D_{21}}{\partial \omega_{1}} \frac{\partial \omega_{1}}{\partial z}\right)+\left(\frac{1-\omega_{2}}{\left(1-\omega_{1}-\omega_{2}\right)^{2}}\right)\left(\frac{\partial D_{11}}{\partial \omega_{1}} \frac{\partial \omega_{1}}{\partial z}+\frac{\partial D_{12}}{\partial \omega_{1}} \frac{\partial \omega_{2}}{\partial z}\right)
\end{array}\right\} \frac{\partial \omega_{1}}{\partial z}+ \\
& \left\{\begin{array}{l}
\left(\frac{2 \omega_{1}}{\left(1-\omega_{1}-\omega_{2}\right)^{3}}+\frac{1}{\left(1-\omega_{1}-\omega_{2}\right)^{2}}\right)\left(D_{22} \frac{\partial \omega_{2}}{\partial z}+D_{21} \frac{\partial \omega_{1}}{\partial z}\right)+\left(\frac{2\left(1-\omega_{2}\right)}{\left(1-\omega_{1}-\omega_{2}\right)^{3}}-\frac{1}{\left(1-\omega_{1}-\omega_{2}\right)^{2}}\right)\left(D_{11} \frac{\partial \omega_{1}}{\partial z}+D_{12} \frac{\partial \omega_{2}}{\partial z}\right)+ \\
\left(\frac{\omega_{1}}{\left(1-\omega_{1}-\omega_{2}\right)^{2}}\right)\left(\frac{\partial D_{22}}{\partial \omega_{1}} \frac{\partial \omega_{2}}{\partial z}+\frac{\partial D_{21}}{\partial \omega_{1}} \frac{\partial \omega_{1}}{\partial z}\right)+\left(\frac{1-\omega_{2}}{\left(1-\omega_{1}-\omega_{2}\right)^{2}}-\frac{1}{1-\omega_{1}-\omega_{2}}\right)\left(\frac{\partial D_{11}}{\partial \omega_{1}} \frac{\partial \omega_{1}}{\partial z}+\frac{\partial D_{12}}{\partial \omega_{1}} \frac{\partial \omega_{2}}{\partial z}\right)
\end{array}\right\} \frac{\partial \omega_{2}}{\partial z}+ \\
& \left\{D_{21}\left(\frac{\omega_{1}}{\left(1-\omega_{1}-\omega_{2}\right)^{2}}+\frac{1}{\left(1-\omega_{1}-\omega_{2}\right)}\right)+\frac{\left(1-\omega_{2}\right)}{\left(1-\omega_{1}-\omega_{2}\right)^{2}} D_{11}+\frac{\partial D_{21}}{\partial \omega_{1}} \frac{\omega_{1}}{\left(1-\omega_{1}-\omega_{2}\right)}+\frac{\left(1-\omega_{2}\right)}{\left(1-\omega_{1}-\omega_{2}\right)} \frac{\partial D_{11}}{\partial \omega_{1}}\right\}\left(\frac{\partial^{2} \omega_{1}}{\partial z^{2}}\right)+ \\
& \left\{D_{22}\left(\frac{\omega_{1}}{\left(1-\omega_{1}-\omega_{2}\right)^{2}}+\frac{1}{\left(1-\omega_{1}-\omega_{2}\right)}\right)+D_{21} \frac{\left(1-\omega_{2}\right)}{\left(1-\omega_{1}-\omega_{2}\right)^{2}}+\frac{\partial D_{22}}{\partial \omega_{1}} \frac{\omega_{1}}{\left(1-\omega_{1}-\omega_{2}\right)}+\frac{\partial D_{21}}{\partial \omega_{1}} \frac{\left(1-\omega_{2}\right)}{\left(1-\omega_{1}-\omega_{2}\right)}\right\}\left(\frac{\partial^{2} \omega_{2}}{\partial z^{2}}\right)+ \\
& \left.\int\left(\frac{\omega_{1}}{\left(1-\omega_{1}-\omega_{2}\right)^{2}}+\frac{1}{\left(1-\omega_{1}-\omega_{2}\right)}\right)\left(\frac{\partial D_{22}}{\partial \omega_{1}}\right)+\left(\frac{\omega_{1}}{\left(1-\omega_{1}-\omega_{2}\right)^{2}}+\frac{1}{\left(1-\omega_{1}-\omega_{2}\right)}\right)\left(\frac{\partial D_{21}}{\partial \omega_{2}}\right)+\frac{\left(1-\omega_{2}\right)}{\left(1-\omega_{1}-\omega_{2}\right)^{2}}\left(\frac{\partial D_{11}}{\partial \omega_{2}}\right)+\right) \\
& \left\{\begin{array}{l}
\frac{\left(1-\omega_{2}\right)}{\left(1-\omega_{1}-\omega_{2}\right)^{2}}\left(\frac{\partial D_{12}}{\partial \omega_{1}}\right)+ \\
\frac{\omega_{1}}{\left(1-\omega_{1}-\omega_{2}\right)} \frac{\partial}{\partial \omega_{1}}\left(\frac{\partial D_{22}}{\partial \omega_{1}}\right)+\frac{\omega_{1}}{\left(1-\omega_{1}-\omega_{2}\right)} \frac{\partial}{\partial \omega_{1}}\left(\frac{\partial D_{21}}{\partial \omega_{2}}\right)+\frac{\left(1-\omega_{2}\right)}{\left(1-\omega_{1}-\omega_{2}\right)} \frac{\partial}{\partial \omega_{1}}\left(\frac{\partial D_{11}}{\partial \omega_{2}}\right)+\frac{\left(1-\omega_{2}\right)}{\left(1-\omega_{1}-\omega_{2}\right)} \frac{\partial}{\partial \omega_{1}}\left(\frac{\partial D_{12}}{\partial \omega_{1}}\right)
\end{array}\right\} \\
& +\left\{\frac{\left(1-\omega_{2}\right)}{\left(1-\omega_{1}-\omega_{2}\right)^{2}}\left(\frac{\partial D_{21}}{\partial \omega_{1}}\right)+\frac{\left(1-\omega_{2}\right)}{\left(1-\omega_{1}-\omega_{2}\right)^{2}}\left(\frac{\partial D_{11}}{\partial \omega_{1}}\right)+\frac{\omega_{1}}{\left(1-\omega_{1}-\omega_{2}\right)} \frac{\partial}{\partial \omega_{1}}\left(\frac{\partial D_{21}}{\partial \omega_{1}}\right)+\frac{\left(1-\omega_{2}\right)}{\left(1-\omega_{1}-\omega_{2}\right)} \frac{\partial}{\partial \omega_{1}}\left(\frac{\partial D_{11}}{\partial \omega_{1}}\right)\right\}\left\{\left(\frac{\partial \omega_{1}}{\partial z}\right)^{2}\right. \\
& +\left\{\frac{\left(1-\omega_{2}\right)}{\left(1-\omega_{1}-\omega_{2}\right)^{2}}\left(\frac{\partial D_{12}}{\partial \omega_{2}}\right)+\frac{\left(1-\omega_{2}\right)}{\left(1-\omega_{1}-\omega_{2}\right)^{2}}\left(\frac{\partial D_{22}}{\partial \omega_{2}}\right)+\frac{1-\omega_{2}}{\left(1-\omega_{1}-\omega_{2}\right)} \frac{\partial}{\partial \omega_{1}}\left(\frac{\partial D_{12}}{\partial \omega_{2}}\right)+\frac{\omega_{1}}{\left(1-\omega_{1}-\omega_{2}\right)} \frac{\partial}{\partial \omega_{1}}\left(\frac{\partial D_{22}}{\partial \omega_{2}}\right)\right\}\left(\frac{\partial \omega_{2}}{\partial z}\right)^{2}
\end{aligned}
$$


Differentiating $f_{1}$ w.r.t $\omega_{1 z}$, we get

$$
\begin{aligned}
& \frac{\partial f_{1}}{\partial \omega_{12}}=\left\{\begin{array}{l}
\left.\left(\frac{\omega_{1}}{\left(1-\omega_{1}-\omega_{2}\right)^{2}}+\frac{1}{1-\omega_{1}-\omega_{2}}\right)\left(D_{22} \frac{\partial \omega_{2}}{\partial z}+D_{21} \frac{\partial \omega_{1}}{\partial z}\right)+\left(\frac{1-\omega_{2}}{\left(1-\omega_{1}-\omega_{2}\right)^{2}}\right)\left(D_{11} \frac{\partial \omega_{1}}{\partial z}+D_{12} \frac{\partial \omega_{2}}{\partial z}\right)+\right\} \\
\left\{\left(\frac{\omega_{1}}{\left(1-\omega_{1}-\omega_{2}\right)^{2}}+\frac{1}{1-\omega_{1}-\omega_{2}}\right)\left(D_{21}\right)+\left(\frac{1-\omega_{2}}{\left(1-\omega_{1}-\omega_{2}\right)^{2}}\right)\left(D_{11}\right)\right] \frac{\partial \omega_{1}}{\partial z}
\end{array}\right\} \\
& \left\{\left(\frac{\omega_{1}}{\left(1-\omega_{1}-\omega_{2}\right)^{2}}\right)\left(D_{21}\right)+\left(\frac{1-\omega_{2}}{\left(1-\omega_{1}-\omega_{2}\right)^{2}}-\frac{1}{1-\omega_{1}-\omega_{2}}\right)\left(D_{11}\right)\right\} \frac{\partial \omega_{2}}{\partial z}+ \\
& \left\{\frac{\omega_{1}}{\left(1-\omega_{1}-\omega_{2}\right)}\left(\frac{\partial D_{22}}{\partial \omega_{1}}\right)+\frac{\omega_{1}}{\left(1-\omega_{1}-\omega_{2}\right)}\left(\frac{\partial D_{21}}{\partial \omega_{2}}\right)+\frac{\left(1-\omega_{2}\right)}{\left(1-\omega_{1}-\omega_{2}\right)}\left(\frac{\partial D_{11}}{\partial \omega_{2}}\right)+\frac{\left(1-\omega_{2}\right)}{\left(1-\omega_{1}-\omega_{2}\right)}\left(\frac{\partial D_{12}}{\partial \omega_{1}}\right)\right\}\left(\frac{\partial \omega_{2}}{\partial z}\right)+ \\
& +2\left\{\frac{\omega_{1}}{\left(1-\omega_{1}-\omega_{2}\right)}\left(\frac{\partial D_{21}}{\partial \omega_{1}}\right)+\frac{\left(1-\omega_{2}\right)}{\left(1-\omega_{1}-\omega_{2}\right)}\left(\frac{\partial D_{11}}{\partial \omega_{1}}\right)\right\} \frac{\partial \omega_{1}}{\partial z}
\end{aligned}
$$

Differentiating $f_{1}$ w.r.t $\omega_{1 z z}$, we get

$$
\frac{\partial \mathrm{f}_{1}}{\partial \omega_{1 \mathrm{zz}}}=\left\{\mathrm{D}_{21} \frac{\omega_{1}}{\left(1-\omega_{1}-\omega_{2}\right)}+\frac{\left(1-\omega_{2}\right)}{\left(1-\omega_{1}-\omega_{2}\right)} \mathrm{D}_{11}\right\}
$$

Taking second derivative of $\frac{\partial f}{\partial \omega_{1 z}}$ w.r.t $z$

$$
\begin{aligned}
& \frac{\partial}{\partial z}\left(\frac{\partial f_{1}}{\partial \omega_{12}}\right)=\left(\frac{\omega_{1}}{\left(1-\omega_{1}-\omega_{2}\right)^{2}}+\frac{1}{1-\omega_{1}-\omega_{2}}\right)\left(\frac{\partial D_{22}}{\partial z} \frac{\partial \omega_{2}}{\partial z}+\frac{\partial D_{21}}{\partial z} \frac{\partial \omega_{1}}{\partial z}\right)+ \\
& \left(\left(\frac{2 \omega_{1}}{\left(1-\omega_{1}-\omega_{2}\right)^{3}}+\frac{1}{\left(1-\omega_{1}-\omega_{2}\right)^{2}}+\frac{1}{\left(1-\omega_{1}-\omega_{2}\right)^{2}}\right) \frac{\partial \omega_{1}}{\partial z}+\frac{2 \omega_{1}}{\left(1-\omega_{1}-\omega_{2}\right)^{3}}+\frac{1}{\left(1-\omega_{1}-\omega_{2}\right)^{2}} \frac{\partial \omega_{2}}{\partial z}\right)\left(D_{22} \frac{\partial \omega_{2}}{\partial z}+D_{21} \frac{\partial \omega_{1}}{\partial z}\right) \\
& +\left(\frac{1-\omega_{2}}{\left(1-\omega_{1}-\omega_{2}\right)^{2}}\right)\left(\frac{\partial D_{11}}{\partial z} \frac{\partial \omega_{1}}{\partial z}+\frac{\partial D_{12}}{\partial z} \frac{\partial \omega_{2}}{\partial z}\right)+\left(\frac{2\left(1-\omega_{2}\right)}{\left(1-\omega_{1}-\omega_{2}\right)^{3}} \frac{\partial \omega_{1}}{\partial z}+\left(\frac{2\left(1-\omega_{2}\right)}{\left(1-\omega_{1}-\omega_{2}\right)^{3}}-\frac{1}{\left(1-\omega_{1}-\omega_{2}\right)^{2}}\right) \frac{\partial \omega_{2}}{\partial z}\right) \\
& \left(D_{11} \frac{\partial \omega_{1}}{\partial z}+D_{12} \frac{\partial \omega_{2}}{\partial z}\right) \\
& \left(\frac{\omega_{1}}{\left(1-\omega_{1}-\omega_{2}\right)^{2}}+\frac{1}{1-\omega_{1}-\omega_{2}}\right)\left(\frac{\partial D_{21}}{\partial z}\right) \frac{\partial \omega_{1}}{\partial z}+
\end{aligned}
$$




$$
\begin{aligned}
& \left(\begin{array}{l}
\left(\frac{2 \omega_{1}}{\left(1-\omega_{1}-\omega_{2}\right)^{3}}+\frac{1}{\left(1-\omega_{1}-\omega_{2}\right)^{2}}+\frac{1}{\left(1-\omega_{1}-\omega_{2}\right)^{2}}\right) \frac{\partial \omega_{1}}{\partial z}+ \\
\left(\frac{2 \omega_{1}}{\left(1-\omega_{1}-\omega_{2}\right)^{3}} \frac{1}{\left(1-\omega_{1}-\omega_{2}\right)^{2}}\right) \frac{\partial \omega_{2}}{\partial z}
\end{array}\right) D_{21} \frac{\partial \omega_{1}}{\partial z} \\
& \left(\frac{1-\omega_{2}}{\left(1-\omega_{1}-\omega_{2}\right)^{2}}\right)\left(\frac{\partial D_{11}}{\partial z}\right) \frac{\partial \omega_{1}}{\partial z}+\left[\left(\frac{2\left(1-\omega_{2}\right)}{\left(1-\omega_{1}-\omega_{2}\right)^{3}} \frac{\partial \omega_{1}}{\partial z}+\left(\frac{2\left(1-\omega_{2}\right)}{\left(1-\omega_{1}-\omega_{2}\right)^{3}}-\frac{1}{\left(1-\omega_{1}-\omega_{2}\right)^{2}}\right) \frac{\partial \omega_{2}}{\partial z}\right)\right] D_{11} \frac{\partial \omega_{1}}{\partial z} \\
& \left(\frac{\omega_{1}}{\left(1-\omega_{1}-\omega_{2}\right)^{2}}\right)\left(\frac{\partial D_{21}}{\partial z}\right) \frac{\partial \omega_{2}}{\partial z}+\left[\left(\frac{2 \omega_{1}}{\left(1-\omega_{1}-\omega_{2}\right)^{3}}+\frac{1}{\left(1-\omega_{1}-\omega_{2}\right)^{2}}\right) \frac{\partial \omega_{1}}{\partial z}+\left(\frac{2 \omega_{1}}{\left(1-\omega_{1}-\omega_{2}\right)^{3}}\right) \frac{\partial \omega_{2}}{\partial z}\right] D_{21} \frac{\partial \omega_{2}}{\partial z} \\
& \left(\frac{1-\omega_{2}}{\left(1-\omega_{1}-\omega_{2}\right)^{2}}-\frac{1}{1-\omega_{1}-\omega_{2}}\right)\left(\frac{\partial D_{11}}{\partial z}\right) \frac{\partial \omega_{2}}{\partial z} \\
& +\left(\left(\frac{2\left(1-\omega_{2}\right)}{\left(1-\omega_{1}-\omega_{2}\right)^{3}}-\frac{1}{\left(1-\omega_{1}-\omega_{2}\right)^{2}}\right) \frac{\partial \omega_{1}}{\partial z}+\left(\frac{2\left(1-\omega_{2}\right)}{\left(1-\omega_{1}-\omega_{2}\right)^{3}}-\frac{1}{\left(1-\omega_{1}-\omega_{2}\right)^{2}} \frac{1}{\left(1-\omega_{1}-\omega_{2}\right)^{2}}\right) \frac{\partial \omega_{2}}{\partial z}\right) D_{11} \frac{\partial \omega_{2}}{\partial z} \\
& \frac{\omega_{1}}{\left(1-\omega_{1}-\omega_{2}\right)} \frac{\partial}{\partial z}\left(\frac{\partial D_{22}}{\partial \omega_{1}}\right)\left(\frac{\partial \omega_{2}}{\partial z}\right)+\left(\left(\frac{\omega_{1}}{\left(1-\omega_{1}-\omega_{2}\right)^{2}}+\frac{1}{\left(1-\omega_{1}-\omega_{2}\right)}\right) \frac{\partial \omega_{1}}{\partial z}+\left(\frac{\omega_{1}}{\left(1-\omega_{1}-\omega_{2}\right)^{2}}\right) \frac{\partial \omega_{2}}{\partial z}\right)\left(\frac{\partial D_{22}}{\partial \omega_{1}}\right)\left(\frac{\partial \omega_{2}}{\partial z}\right) \\
& \frac{\omega_{1}}{\left(1-\omega_{1}-\omega_{2}\right)} \frac{\partial}{\partial z}\left(\frac{\partial D_{21}}{\partial \omega_{2}}\right)\left(\frac{\partial \omega_{2}}{\partial z}\right)+\left(\left(\frac{\omega_{1}}{\left(1-\omega_{1}-\omega_{2}\right)^{2}}+\frac{1}{\left(1-\omega_{1}-\omega_{2}\right)}\right) \frac{\partial \omega_{1}}{\partial z}+\left(\frac{\omega_{1}}{\left(1-\omega_{1}-\omega_{2}\right)^{2}}\right) \frac{\partial \omega_{2}}{\partial z}\right)\left(\frac{\partial D_{21}}{\partial \omega_{2}}\right)\left(\frac{\partial \omega_{2}}{\partial z}\right) \\
& \frac{\left(1-\omega_{2}\right)}{\left(1-\omega_{1}-\omega_{2}\right)} \frac{\partial}{\partial z}\left(\frac{\partial D_{11}}{\partial \omega_{2}}\right)\left(\frac{\partial \omega_{2}}{\partial z}\right)+\left(\left(\frac{\left(1-\omega_{2}\right)}{\left(1-\omega_{1}-\omega_{2}\right)^{2}}\right) \frac{\partial \omega_{1}}{\partial z}+\left(\frac{\left(1-\omega_{2}\right)}{\left(1-\omega_{1}-\omega_{2}\right)^{2}}-\frac{1}{\left(1-\omega_{1}-\omega_{2}\right)}\right) \frac{\partial \omega_{2}}{\partial z}\right)\left(\frac{\partial D_{11}}{\partial \omega_{2}}\right)\left(\frac{\partial \omega_{2}}{\partial z}\right) \\
& \frac{\left(1-\omega_{2}\right)}{\left(1-\omega_{1}-\omega_{2}\right)} \frac{\partial}{\partial z}\left(\frac{\partial D_{12}}{\partial \omega_{1}}\right)\left(\frac{\partial \omega_{2}}{\partial z}\right)+\left(\left(\frac{\left(1-\omega_{2}\right)}{\left(1-\omega_{1}-\omega_{2}\right)^{2}}\right) \frac{\partial \omega_{1}}{\partial z}+\left(\frac{\left(1-\omega_{2}\right)}{\left(1-\omega_{1}-\omega_{2}\right)^{2}}-\frac{1}{\left(1-\omega_{1}-\omega_{2}\right)}\right) \frac{\partial \omega_{2}}{\partial z}\right)\left(\frac{\partial D_{12}}{\partial \omega_{1}}\right)\left(\frac{\partial \omega_{2}}{\partial z}\right) \\
& +2 \frac{\omega_{1}}{\left(1-\omega_{1}-\omega_{2}\right)}\left(\frac{\partial D_{21}}{\partial \omega_{1}}\right)\left(\frac{\partial^{2} \omega_{1}}{\partial z^{2}}\right)+2 \frac{\left(1-\omega_{2}\right)}{\left(1-\omega_{1}-\omega_{2}\right)}\left(\frac{\partial D_{11}}{\partial \omega_{1}}\right)\left(\frac{\partial^{2} \omega_{1}}{\partial z^{2}}\right)+ \\
& 2\left(\begin{array}{l}
\left(\frac{\omega_{1}}{\left(1-\omega_{1}-\omega_{2}\right)^{2}}+\frac{1}{\left(1-\omega_{1}-\omega_{2}\right)}\right) \frac{\partial \omega_{1}}{\partial z}+ \\
\left(\frac{\omega_{1}}{\left(1-\omega_{1}-\omega_{2}\right)^{2}}\right) \frac{\partial \omega_{2}}{\partial z}
\end{array}\right)\left(\frac{\partial D_{21}}{\partial \omega_{1}}\right)\left(\frac{\partial \omega_{1}}{\partial z}\right)+ \\
& 2\left(\left(\frac{\left(1-\omega_{2}\right)}{\left(1-\omega_{1}-\omega_{2}\right)^{2}}\right) \frac{\partial \omega_{1}}{\partial z}+\left(\frac{\left(1-\omega_{2}\right)}{\left(1-\omega_{1}-\omega_{2}\right)^{2}}-\frac{1}{\left(1-\omega_{1}-\omega_{2}\right)}\right) \frac{\partial \omega_{2}}{\partial z}\right)\left(\frac{\partial D_{11}}{\partial \omega_{1}}\right)\left(\frac{\partial \omega_{1}}{\partial z}\right)
\end{aligned}
$$

We can expand $\frac{\partial}{\partial z}\left(\frac{\partial D_{22}}{\partial \omega_{1}}\right)$ as follows 


$$
\begin{aligned}
& \frac{\partial}{\partial z}\left(\frac{\partial D_{22}}{\partial \omega_{1}}\right)=\frac{\partial}{\partial \omega_{1}}\left(\frac{\partial D_{22}}{\partial z}\right)=\frac{\partial}{\partial \omega_{1}}\left(\frac{\partial D_{22}}{\partial \omega_{1}} \frac{\partial \omega_{1}}{\partial z}+\frac{\partial D_{22}}{\partial \omega_{2}} \frac{\partial \omega_{2}}{\partial z}\right)=\frac{\partial^{2} D_{22}}{\partial \omega_{1}{ }^{2}} \frac{\partial \omega_{1}}{\partial z}+\frac{\partial D_{22}}{\partial \omega_{1}} \frac{\partial}{\partial \omega_{1}}\left(\frac{\partial \omega_{1}}{\partial z}\right)+ \\
& \frac{\partial^{2} D_{22}}{\partial \omega_{1} \omega_{2}} \frac{\partial \omega_{2}}{\partial z}+\frac{\partial D_{22}}{\partial \omega_{2}} \frac{\partial}{\partial \omega_{1}}\left(\frac{\partial \omega_{2}}{\partial z}\right)
\end{aligned}
$$

$\frac{\partial}{\partial z}\left(\frac{\partial D_{22}}{\partial \omega_{1}}\right)=\frac{\partial}{\partial \omega_{1}}\left(\frac{\partial D_{22}}{\partial z}\right)=\frac{\partial}{\partial \omega_{1}}\left(\frac{\partial D_{22}}{\partial \omega_{1}} \frac{\partial \omega_{1}}{\partial z}+\frac{\partial D_{22}}{\partial \omega_{2}} \frac{\partial \omega_{2}}{\partial z}\right)=\frac{\partial^{2} D_{22}}{\partial \omega_{1}^{2}} \frac{\partial \omega_{1}}{\partial z}+\frac{\partial^{2} D_{22}}{\partial \omega_{1} \omega_{2}} \frac{\partial \omega_{2}}{\partial z}$

Similarly other terms can also be expanded. Substituting their values in Equation (4.58) we get

$$
\begin{aligned}
& \frac{\partial}{\partial z}\left(\frac{\partial f}{\partial \omega_{1 z}}\right)=\left(\frac{\omega_{1}}{\left(1-\omega_{1}-\omega_{2}\right)^{2}}+\frac{1}{1-\omega_{1}-\omega_{2}}\right)\left(\begin{array}{l}
\left(\frac{\partial D_{22}}{\partial \omega_{1}} \frac{\partial \omega_{1}}{\partial z}+\frac{\partial D_{22}}{\partial \omega_{2}} \frac{\partial \omega_{2}}{\partial z}\right) \frac{\partial \omega_{2}}{\partial z}+ \\
\left(\frac{\partial D_{21}}{\partial \omega_{1}} \frac{\partial \omega_{1}}{\partial z}+\frac{\partial D_{21}}{\partial \omega_{2}} \frac{\partial \omega_{2}}{\partial z}\right) \frac{\partial \omega_{1}}{\partial z}
\end{array}\right)+ \\
& \left(\left(\frac{2 \omega_{1}}{\left(1-\omega_{1}-\omega_{2}\right)^{3}}+\frac{1}{\left(1-\omega_{1}-\omega_{2}\right)^{2}}+\frac{1}{\left(1-\omega_{1}-\omega_{2}\right)^{2}}\right) \frac{\partial \omega_{1}}{\partial z}+\frac{2 \omega_{1}}{\left(1-\omega_{1}-\omega_{2}\right)^{3}}+\frac{1}{\left(1-\omega_{1}-\omega_{2}\right)^{2}} \frac{\partial \omega_{2}}{\partial z}\right) \\
& \left(D_{22} \frac{\partial \omega_{2}}{\partial z}+D_{21} \frac{\partial \omega_{1}}{\partial z}\right) \\
& +\left(\frac{1-\omega_{2}}{\left(1-\omega_{1}-\omega_{2}\right)^{2}}\right)\left(\left(\frac{\partial D_{11}}{\partial \omega_{1}} \frac{\partial \omega_{1}}{\partial z}+\frac{\partial D_{11}}{\partial \omega_{2}} \frac{\partial \omega_{2}}{\partial z}\right) \frac{\partial \omega_{1}}{\partial z}+\left(\frac{\partial D_{12}}{\partial \omega_{1}} \frac{\partial \omega_{1}}{\partial z}+\frac{\partial D_{12}}{\partial \omega_{2}} \frac{\partial \omega_{2}}{\partial z}\right) \frac{\partial \omega_{2}}{\partial z}\right)+ \\
& \left(\begin{array}{l}
\frac{2\left(1-\omega_{2}\right)}{\left(1-\omega_{1}-\omega_{2}\right)^{3}} \frac{\partial \omega_{1}}{\partial z}+ \\
\left(\frac{2\left(1-\omega_{2}\right)}{\left(1-\omega_{1}-\omega_{2}\right)^{3}}-\frac{1}{\left(1-\omega_{1}-\omega_{2}\right)^{2}}\right) \frac{\partial \omega_{2}}{\partial z}
\end{array}\right)\left(D_{11} \frac{\partial \omega_{1}}{\partial z}+D_{12} \frac{\partial \omega_{2}}{\partial z}\right) \\
& \left(\frac{\omega_{1}}{\left(1-\omega_{1}-\omega_{2}\right)^{2}}+\frac{1}{1-\omega_{1}-\omega_{2}}\right)\left(\frac{\partial D_{21}}{\partial \omega_{1}} \frac{\partial \omega_{1}}{\partial z}+\frac{\partial D_{21}}{\partial \omega_{2}} \frac{\partial \omega_{2}}{\partial z}\right) \frac{\partial \omega_{1}}{\partial z}+
\end{aligned}
$$




$$
\begin{aligned}
& \left(\begin{array}{l}
\left(\frac{2 \omega_{1}}{\left(1-\omega_{1}-\omega_{2}\right)^{3}}+\frac{1}{\left(1-\omega_{1}-\omega_{2}\right)^{2}}+\frac{1}{\left(1-\omega_{1}-\omega_{2}\right)^{2}}\right) \frac{\partial \omega_{1}}{\partial z}+ \\
\left(\frac{2 \omega_{1}}{\left(1-\omega_{1}-\omega_{2}\right)^{3}} \frac{1}{\left(1-\omega_{1}-\omega_{2}\right)^{2}}\right) \frac{\partial \omega_{2}}{\partial z}
\end{array}\right) D_{21} \frac{\partial \omega_{1}}{\partial z} \\
& \left(\frac{1-\omega_{2}}{\left(1-\omega_{1}-\omega_{2}\right)^{2}}\right)\left(\frac{\partial D_{11}}{\partial \omega_{1}} \frac{\partial \omega_{1}}{\partial z}+\frac{\partial D_{11}}{\partial \omega_{2}} \frac{\partial \omega_{2}}{\partial z}\right) \frac{\partial \omega_{1}}{\partial z}+\left[\left(\frac{2\left(1-\omega_{2}\right)}{\left(1-\omega_{1}-\omega_{2}\right)^{3}} \frac{\partial \omega_{1}}{\partial z}+\left(\frac{2\left(1-\omega_{2}\right)}{\left(1-\omega_{1}-\omega_{2}\right)^{3}}-\frac{1}{\left(1-\omega_{1}-\omega_{2}\right)^{2}}\right) \frac{\partial \omega_{2}}{\partial z}\right)\right] D_{11} \frac{\partial \omega_{1}}{\partial z} \\
& \left(\frac{\omega_{1}}{\left(1-\omega_{1}-\omega_{2}\right)^{2}}\right)\left(\frac{\partial D_{21}}{\partial \omega_{1}} \frac{\partial \omega_{1}}{\partial z}+\frac{\partial D_{21}}{\partial \omega_{2}} \frac{\partial \omega_{2}}{\partial z}\right) \frac{\partial \omega_{2}}{\partial z}+\left[\left(\frac{2 \omega_{1}}{\left(1-\omega_{1}-\omega_{2}\right)^{3}}+\frac{1}{\left(1-\omega_{1}-\omega_{2}\right)^{2}}\right) \frac{\partial \omega_{1}}{\partial z}+\left(\frac{2 \omega_{1}}{\left(1-\omega_{1}-\omega_{2}\right)^{3}}\right) \frac{\partial \omega_{2}}{\partial z}\right] D_{21} \frac{\partial \omega_{2}}{\partial z} \\
& \left(\frac{1-\omega_{2}}{\left(1-\omega_{1}-\omega_{2}\right)^{2}}-\frac{1}{1-\omega_{1}-\omega_{2}}\right)\left(\frac{\partial D_{11}}{\partial \omega_{1}} \frac{\partial \omega_{1}}{\partial z}+\frac{\partial D_{11}}{\partial \omega_{2}} \frac{\partial \omega_{2}}{\partial z}\right) \frac{\partial \omega_{2}}{\partial z}+ \\
& \left(\left(\frac{2\left(1-\omega_{2}\right)}{\left(1-\omega_{1}-\omega_{2}\right)^{3}}-\frac{1}{\left(1-\omega_{1}-\omega_{2}\right)^{2}}\right) \frac{\partial \omega_{1}}{\partial z}+\left(\frac{2\left(1-\omega_{2}\right)}{\left(1-\omega_{1}-\omega_{2}\right)^{3}}-\frac{1}{\left(1-\omega_{1}-\omega_{2}\right)^{2}} \frac{1}{\left(1-\omega_{1}-\omega_{2}\right)^{2}}\right) \frac{\partial \omega_{2}}{\partial z}\right) \\
& D_{11} \frac{\partial \omega_{2}}{\partial z} \\
& \frac{\omega_{1}}{\left(1-\omega_{1}-\omega_{2}\right)} \frac{\partial}{\partial z}\left(\frac{\partial D_{22}}{\partial \omega_{1}}\right)\left(\frac{\partial \omega_{2}}{\partial z}\right)+\left(\left(\frac{\omega_{1}}{\left(1-\omega_{1}-\omega_{2}\right)^{2}}+\frac{1}{\left(1-\omega_{1}-\omega_{2}\right)}\right) \frac{\partial \omega_{1}}{\partial z}+\left(\frac{\omega_{1}}{\left(1-\omega_{1}-\omega_{2}\right)^{2}}\right) \frac{\partial \omega_{2}}{\partial z}\right) \\
& \left(\frac{\partial D_{22}}{\partial \omega_{1}}\right)\left(\frac{\partial \omega_{2}}{\partial z}\right) \\
& \frac{\omega_{1}}{\left(1-\omega_{1}-\omega_{2}\right)} \frac{\partial}{\partial z}\left(\frac{\partial D_{21}}{\partial \omega_{2}}\right)\left(\frac{\partial \omega_{2}}{\partial z}\right)+\left(\left(\frac{\omega_{1}}{\left(1-\omega_{1}-\omega_{2}\right)^{2}}+\frac{1}{\left(1-\omega_{1}-\omega_{2}\right)}\right) \frac{\partial \omega_{1}}{\partial z}+\left(\frac{\omega_{1}}{\left(1-\omega_{1}-\omega_{2}\right)^{2}}\right) \frac{\partial \omega_{2}}{\partial z}\right) \\
& \left(\frac{\partial D_{21}}{\partial \omega_{2}}\right)\left(\frac{\partial \omega_{2}}{\partial z}\right) \\
& \frac{\left(1-\omega_{2}\right)}{\left(1-\omega_{1}-\omega_{2}\right)} \frac{\partial}{\partial z}\left(\frac{\partial D_{11}}{\partial \omega_{2}}\right)\left(\frac{\partial \omega_{2}}{\partial z}\right) \\
& \left(\left(\frac{\left(1-\omega_{2}\right)}{\left(1-\omega_{1}-\omega_{2}\right)^{2}}\right) \frac{\partial \omega_{1}}{\partial z}+\left(\frac{\left(1-\omega_{2}\right)}{\left(1-\omega_{1}-\omega_{2}\right)^{2}}-\frac{1}{\left(1-\omega_{1}-\omega_{2}\right)}\right) \frac{\partial \omega_{2}}{\partial z}\right)\left(\frac{\partial D_{11}}{\partial \omega_{2}}\right)\left(\frac{\partial \omega_{2}}{\partial z}\right) \\
& \frac{\left(1-\omega_{2}\right)}{\left(1-\omega_{1}-\omega_{2}\right)} \frac{\partial}{\partial z}\left(\frac{\partial D_{12}}{\partial \omega_{1}}\right)\left(\frac{\partial \omega_{2}}{\partial z}\right)+\left(\begin{array}{l}
\left(\frac{\left(1-\omega_{2}\right)}{\left(1-\omega_{1}-\omega_{2}\right)^{2}}\right) \frac{\partial \omega_{1}}{\partial z}+ \\
\left(\frac{\left(1-\omega_{2}\right)}{\left(1-\omega_{1}-\omega_{2}\right)^{2}}-\frac{1}{\left(1-\omega_{1}-\omega_{2}\right)}\right) \frac{\partial \omega_{2}}{\partial z}
\end{array}\right) \\
& \left(\frac{\partial D_{12}}{\partial \omega_{1}}\right)\left(\frac{\partial \omega_{2}}{\partial z}\right)+
\end{aligned}
$$




$$
\begin{aligned}
& 2 \frac{\omega_{1}}{\left(1-\omega_{1}-\omega_{2}\right)}\left(\frac{\partial D_{21}}{\partial \omega_{1}}\right)\left(\frac{\partial^{2} \omega_{1}}{\partial z^{2}}\right)+2 \frac{\left(1-\omega_{2}\right)}{\left(1-\omega_{1}-\omega_{2}\right)}\left(\frac{\partial D_{11}}{\partial \omega_{1}}\right)\left(\frac{\partial^{2} \omega_{1}}{\partial z^{2}}\right)+ \\
& \left.2\left(\frac{\omega_{1}}{\left(1-\omega_{1}-\omega_{2}\right)^{2}}+\frac{1}{\left(1-\omega_{1}-\omega_{2}\right)}\right) \frac{\partial \omega_{1}}{\partial z}+\right)\left(\frac{\partial D_{21}}{\partial \omega_{1}}\right)\left(\frac{\partial \omega_{1}}{\partial z}\right)+ \\
& 2\left(\frac{\omega_{1}}{\left(1-\omega_{1}-\omega_{2}\right)^{2}}\right) \frac{\partial \omega_{2}}{\partial z} \\
& \left.2\left(\frac{\left(1-\omega_{2}\right)}{\left(1-\omega_{1}-\omega_{2}\right)^{2}}\right) \frac{\partial \omega_{1}}{\partial z}+\left(\frac{\left(1-\omega_{2}\right)}{\left(1-\omega_{1}-\omega_{2}\right)^{2}}-\frac{1}{\left(1-\omega_{1}-\omega_{2}\right)}\right) \frac{\partial \omega_{2}}{\partial z}\right)\left(\frac{\partial D_{11}}{\partial \omega_{1}}\right)\left(\frac{\partial \omega_{1}}{\partial z}\right)
\end{aligned}
$$

(4.61)

\section{Simplifying Equation (4.61) further we get,}

$$
\begin{aligned}
& \frac{\partial}{\partial z}\left(\frac{\partial f}{\partial \omega_{1 z}}\right)=\left(\frac{\omega_{1}}{\left(1-\omega_{1}-\omega_{2}\right)^{2}}+\frac{1}{1-\omega_{1}-\omega_{2}}\right)\left[\left(\frac{\partial D_{22}}{\partial \omega_{1}} \frac{\partial \omega_{1}}{\partial z}+\frac{\partial D_{22}}{\partial \omega_{2}} \frac{\partial \omega_{2}}{\partial z}\right) \frac{\partial \omega_{2}}{\partial z}+\left(\frac{\partial D_{21}}{\partial \omega_{1}} \frac{\partial \omega_{1}}{\partial z}+\frac{\partial D_{21}}{\partial \omega_{2}} \frac{\partial \omega_{2}}{\partial z}\right) \frac{\partial \omega_{1}}{\partial z}\right]+ \\
& \left(\frac{2 \omega_{1}}{\left(1-\omega_{1}-\omega_{2}\right)^{2}}+\frac{1}{\left(1-\omega_{1}-\omega_{2}\right)^{2}}+\frac{1}{\left(1-\omega_{1}-\omega_{2}\right)^{2}}\right)\left(D_{22} \frac{\partial \omega_{2}}{\partial z}+D_{21} \frac{\partial \omega_{1}}{\partial z}\right) \\
& +\left(\frac{1-\omega_{2}}{\left(1-\omega_{1}-\omega_{2}\right)^{2}}\right)\left(\left(\frac{\partial D_{11}}{\partial \omega_{1}} \frac{\partial \omega_{1}}{\partial z}+\frac{\partial D_{11}}{\partial \omega_{2}} \frac{\partial \omega_{2}}{\partial z}\right) \frac{\partial \omega_{1}}{\partial z}+\left(\frac{\partial D_{12}}{\partial \omega_{1}} \frac{\partial \omega_{1}}{\partial z}+\frac{\partial D_{12}}{\partial \omega_{2}} \frac{\partial \omega_{2}}{\partial z}\right) \frac{\partial \omega_{2}}{\partial z}\right)+ \\
& \left(\frac{2\left(1-\omega_{2}\right)}{\left(1-\omega_{1}-\omega_{2}\right)^{3}}+\frac{2\left(1-\omega_{2}\right)}{\left(1-\omega_{1}-\omega_{2}\right)^{3}}-\frac{1}{\left(1-\omega_{1}-\omega_{2}\right)^{2}}\right)\left(D_{11} \frac{\partial \omega_{1}}{\partial z}+D_{12} \frac{\partial \omega_{2}}{\partial z}\right) \\
& \left(\frac{\omega_{1}}{\left(1-\omega_{1}-\omega_{2}\right)^{2}}+\frac{1}{1-\omega_{1}-\omega_{2}}\right)\left(\left(\frac{\partial D_{21}}{\partial \omega_{1}} \frac{\partial \omega_{1}}{\partial z}+\frac{\partial D_{21}}{\partial \omega_{2}} \frac{\partial \omega_{2}}{\partial z}\right)\right) \frac{\partial \omega_{1}}{\partial z}+ \\
& \left(\frac{2 \omega_{1}}{\left(1-\omega_{1}-\omega_{2}\right)^{2}}+\frac{1}{\left(1-\omega_{1}-\omega_{2}\right)^{2}}+\frac{1}{\left(1-\omega_{1}-\omega_{2}\right)^{2}}\right) D_{21} \frac{\partial \omega_{1}}{\partial z} \\
& \left(\frac{1-\omega_{2}}{\left(1-\omega_{1}-\omega_{2}\right)^{2}}\right)\left(\left(\frac{\partial D_{11}}{\partial \omega_{1}} \frac{\partial \omega_{1}}{\partial z}+\frac{\partial D_{11}}{\partial \omega_{2}} \frac{\partial \omega_{2}}{\partial z}\right)\right) \frac{\partial \omega_{1}}{\partial z}+\left(\frac{2\left(1-\omega_{2}\right)}{\left(1-\omega_{1}-\omega_{2}\right)^{3}}+\frac{2\left(1-\omega_{2}\right)}{\left(1-\omega_{1}-\omega_{2}\right)^{3}}-\frac{1}{\left(1-\omega_{1}-\omega_{2}\right)^{2}}\right) \\
& D_{11} \frac{\partial \omega_{1}}{\partial z} \\
& \left(\frac{\omega_{1}}{\left(1-\omega_{1}-\omega_{2}\right)^{2}}\right)\left(\left(\frac{\partial D_{21}}{\partial \omega_{1}} \frac{\partial \omega_{1}}{\partial z}+\frac{\partial D_{21}}{\partial \omega_{2}} \frac{\partial \omega_{2}}{\partial z}\right)\right) \frac{\partial \omega_{2}}{\partial z}+\left(\frac{2\left(1-\omega_{2}\right)}{\left(1-\omega_{1}-\omega_{2}\right)^{3}}+\frac{2\left(1-\omega_{2}\right)}{\left(1-\omega_{1}-\omega_{2}\right)^{3}}-\frac{1}{\left(1-\omega_{1}-\omega_{2}\right)^{2}}\right) D_{21} \frac{\partial \omega_{2}}{\partial z} \\
& \left(\frac{1-\omega_{2}}{\left(1-\omega_{1}-\omega_{2}\right)^{2}}-\frac{1}{1-\omega_{1}-\omega_{2}}\right)\left(\left(\frac{\partial D_{11}}{\partial \omega_{1}} \frac{\partial \omega_{1}}{\partial z}+\frac{\partial D_{11}}{\partial \omega_{2}} \frac{\partial \omega_{2}}{\partial z}\right)\right) \frac{\partial \omega_{2}}{\partial z}+ \\
& \left(\frac{2 \omega_{1}}{\left(1-\omega_{1}-\omega_{2}\right)^{2}}+\frac{1}{\left(1-\omega_{1}-\omega_{2}\right)^{2}}+\frac{1}{\left(1-\omega_{1}-\omega_{2}\right)^{2}}\right) \\
& D_{11} \frac{\partial \omega_{2}}{\partial z}+
\end{aligned}
$$




$$
\begin{aligned}
& \frac{\omega_{1}}{\left(1-\omega_{1}-\omega_{2}\right)}\left(\frac{\partial^{2} D_{22}}{\partial \omega_{1}^{2}} \frac{\partial \omega_{1}}{\partial z}+\frac{\partial^{2} D_{22}}{\partial \omega_{1} \omega_{2}} \frac{\partial \omega_{2}}{\partial z}\right)\left(\frac{\partial \omega_{2}}{\partial z}\right)+\left(\frac{\omega_{1}}{\left(1-\omega_{1}-\omega_{2}\right)^{2}}+\frac{1}{\left(1-\omega_{1}-\omega_{2}\right)}-\frac{\omega_{1}}{\left(1-\omega_{1}-\omega_{2}\right)^{2}}\right) \\
& \left(\frac{\partial D_{22}}{\partial \omega_{1}}\right)\left(\frac{\partial \omega_{2}}{\partial z}\right) \\
& \frac{\omega_{1}}{\left(1-\omega_{1}-\omega_{2}\right)}\left(\frac{\partial^{2} D_{21}}{\partial \omega_{1} \omega_{2}} \frac{\partial \omega_{1}}{\partial z}+\frac{\partial^{2} D_{21}}{\partial \omega_{2}^{2}} \frac{\partial \omega_{2}}{\partial z}\right)\left(\frac{\partial \omega_{2}}{\partial z}\right)+\left(\frac{\omega_{1}}{\left(1-\omega_{1}-\omega_{2}\right)^{2}}+\frac{1}{\left(1-\omega_{1}-\omega_{2}\right)}-\frac{\omega_{1}}{\left(1-\omega_{1}-\omega_{2}\right)^{2}}\right) \\
& \left(\frac{\partial D_{21}}{\partial \omega_{2}}\right)\left(\frac{\partial \omega_{2}}{\partial z}\right) \\
& \frac{\left(1-\omega_{2}\right)}{\left(1-\omega_{1}-\omega_{2}\right)}\left(\frac{\partial^{2} D_{11}}{\partial \omega_{1} \omega_{2}} \frac{\partial \omega_{1}}{\partial z}+\frac{\partial^{2} D_{11}}{\partial \omega_{2}^{2}} \frac{\partial \omega_{2}}{\partial z}\right)\left(\frac{\partial \omega_{2}}{\partial z}\right)+\left(\frac{\left(1-\omega_{2}\right)}{\left(1-\omega_{1}-\omega_{2}\right)^{2}}+\frac{\left(1-\omega_{2}\right)}{\left(1-\omega_{1}-\omega_{2}\right)^{2}}-\frac{1}{\left(1-\omega_{1}-\omega_{2}\right)}\right) \\
& \left(\frac{\partial D_{11}}{\partial \omega_{2}}\right)\left(\frac{\partial \omega_{2}}{\partial z}\right) \\
& \frac{\left(1-\omega_{2}\right)}{\left(1-\omega_{1}-\omega_{2}\right)}\left(\frac{\partial^{2} D_{12}}{\partial \omega_{1}^{2}} \frac{\partial \omega_{1}}{\partial z}+\frac{\partial^{2} D_{12}}{\partial \omega_{1} \omega_{2}} \frac{\partial \omega_{2}}{\partial z}\right)\left(\frac{\partial \omega_{2}}{\partial z}\right)+\left(\frac{\left(1-\omega_{2}\right)}{\left(1-\omega_{1}-\omega_{2}\right)^{2}}+\frac{\left(1-\omega_{2}\right)}{\left(1-\omega_{1}-\omega_{2}\right)^{2}}-\frac{1}{\left(1-\omega_{1}-\omega_{2}\right)}\right) \\
& \left(\frac{\partial D_{12}}{\partial \omega_{1}}\right)\left(\frac{\partial \omega_{2}}{\partial z}\right) \\
& +2 \frac{\omega_{1}}{\left(1-\omega_{1}-\omega_{2}\right)}\left(\frac{\partial D_{21}}{\partial \omega_{1}}\right)\left(\frac{\partial^{2} \omega_{1}}{\partial z^{2}}\right)+2 \frac{\left(1-\omega_{2}\right)}{\left(1-\omega_{1}-\omega_{2}\right)}\left(\frac{\partial D_{11}}{\partial \omega_{1}}\right)\left(\frac{\partial^{2} \omega_{1}}{\partial z^{2}}\right)+ \\
& 2\left(\frac{\omega_{1}}{\left(1-\omega_{1}-\omega_{2}\right)^{2}}+\frac{1}{\left(1-\omega_{1}-\omega_{2}\right)}-\frac{\omega_{1}}{\left(1-\omega_{1}-\omega_{2}\right)^{2}}\right)\left(\frac{\partial D_{21}}{\partial \omega_{1}}\right)\left(\frac{\partial \omega_{1}}{\partial z}\right)+ \\
& 2\left(\frac{\left(1-\omega_{2}\right)}{\left(1-\omega_{1}-\omega_{2}\right)^{2}}+\frac{\left(1-\omega_{2}\right)}{\left(1-\omega_{1}-\omega_{2}\right)^{2}}-\frac{1}{\left(1-\omega_{1}-\omega_{2}\right)}\right)\left(\frac{\partial D_{11}}{\partial \omega_{1}}\right)\left(\frac{\partial \omega_{1}}{\partial z}\right)
\end{aligned}
$$

Taking second derivative of $\frac{\partial f_{1}}{\partial \omega_{1 z z}}$, Equation (4.57), w.r.t $z$, we get

$$
\begin{aligned}
& \frac{\partial}{\partial z}\left(\frac{\partial f_{1}}{\partial \omega_{12}}\right)=\frac{\omega_{1}}{\left(1-\omega_{1}-\omega_{2}\right)}\left(\frac{\partial D_{21}}{\partial \omega_{1}} \frac{\partial \omega_{1}}{\partial z}+\frac{\partial D_{21}}{\partial \omega_{2}} \frac{\partial \omega_{2}}{\partial z}\right)+\frac{\left(1-\omega_{2}\right)}{\left(1-\omega_{1}-\omega_{2}\right)}\left(\frac{\partial D_{11}}{\partial \omega_{1}} \frac{\partial \omega_{1}}{\partial z}+\frac{\partial D_{11}}{\partial \omega_{2}} \frac{\partial \omega_{2}}{\partial z}\right)+ \\
& \left(\left(\frac{\omega_{1}}{\left(1-\omega_{1}-\omega_{2}\right)^{2}}+\frac{1}{\left(1-\omega_{1}-\omega_{2}\right)}\right) \frac{\partial \omega_{1}}{\partial z}+\left(\frac{\omega_{1}}{\left(1-\omega_{1}-\omega_{2}\right)^{2}}\right) \frac{\partial \omega_{2}}{\partial z}\right) D_{21}+\left(\begin{array}{l}
\left(\frac{\left(1-\omega_{2}\right)}{\left(1-\omega_{1}-\omega_{2}\right)^{2}}+\frac{\left(1-\omega_{2}\right)}{\left(1-\omega_{1}-\omega_{2}\right)^{2}}\right) \frac{\partial \omega_{1}}{\partial z} \\
+\left(\frac{1}{\left(1-\omega_{1}-\omega_{2}\right)}\right) \frac{\partial \omega_{2}}{\partial z}
\end{array}\right) D_{11}
\end{aligned}
$$

67 
Taking third derivative of $\frac{\partial f_{1}}{\partial \omega_{1 z z}}$ w.r.t $z$, we get

$$
\begin{aligned}
& \frac{\partial^{2}}{\partial z^{2}}\left(\frac{\partial f_{1}}{\partial \omega_{1 z}}\right)=\frac{\omega_{1}}{\left(1-\omega_{1}-\omega_{2}\right)}\left(\left(\frac{\partial D_{21}}{\partial \omega_{1}} \frac{\partial^{2} \omega_{1}}{\partial z^{2}}+\frac{\partial \omega_{1}}{\partial z} \frac{\partial^{2} D_{21}}{\partial \omega_{1} \omega_{2}} \frac{\partial \omega_{2}}{\partial z}\right)\left(\frac{\partial \omega_{1}}{\partial z}\right)+\left(\frac{\partial D_{21}}{\partial \omega_{2}} \frac{\partial^{2} \omega_{2}}{\partial z^{2}}+\frac{\partial \omega_{2}}{\partial z} \frac{\partial^{2} D_{21}}{\partial \omega_{1} \omega_{2}} \frac{\partial \omega_{1}}{\partial z}\right)\left(\frac{\partial \omega_{2}}{\partial z}\right)\right)+ \\
& \left(\frac{\partial D_{21}}{\partial \omega_{1}} \frac{\partial \omega_{1}}{\partial z}+\frac{\partial D_{21}}{\partial \omega_{2}} \frac{\partial \omega_{2}}{\partial z}\right) \\
& \left(\left(\frac{\omega_{1}}{\left(1-\omega_{1}-\omega_{2}\right)^{2}}+\frac{1}{\left(1-\omega_{1}-\omega_{2}\right)}\right) \frac{\partial \omega_{1}}{\partial z}+\left(\frac{\omega_{1}}{\left(1-\omega_{1}-\omega_{2}\right)^{2}}\right) \frac{\partial \omega_{2}}{\partial z}\right) \\
& +\frac{\left(1-\omega_{2}\right)}{\left(1-\omega_{1}-\omega_{2}\right)}\left(\left(\frac{\partial D_{11}}{\partial \omega_{1}} \frac{\partial^{2} \omega_{1}}{\partial z^{2}}+\frac{\partial \omega_{1}}{\partial z} \frac{\partial^{2} D_{11}}{\partial \omega_{1} \omega_{2}} \frac{\partial \omega_{2}}{\partial z}\right) \frac{\partial \omega_{1}}{\partial z}+\left(\frac{\partial D_{11}}{\partial \omega_{2}} \frac{\partial^{2} \omega_{2}}{\partial z^{2}}+\frac{\partial \omega_{2}}{\partial z} \frac{\partial^{2} D_{11}}{\partial \omega_{1} \omega_{2}} \frac{\partial \omega_{1}}{\partial z}\right) \frac{\partial \omega_{2}}{\partial z}\right)+ \\
& \left(\left(\frac{\left(1-\omega_{2}\right)}{\left(1-\omega_{1}-\omega_{2}\right)^{2}}\right) \frac{\partial \omega_{1}}{\partial z}+\left(\frac{\left(1-\omega_{2}\right)}{\left(1-\omega_{1}-\omega_{2}\right)^{2}}-\frac{1}{\left(1-\omega_{1}-\omega_{2}\right)}\right) \frac{\partial \omega_{2}}{\partial z}\right)\left(\frac{\partial D_{11}}{\partial \omega_{1}} \frac{\partial \omega_{1}}{\partial z}+\frac{\partial D_{11}}{\partial \omega_{2}} \frac{\partial \omega_{2}}{\partial z}\right)+ \\
& \left(\left(\frac{\omega_{1}}{\left(1-\omega_{1}-\omega_{2}\right)^{2}}+\frac{1}{\left(1-\omega_{1}-\omega_{2}\right)}\right) \frac{\partial \omega_{1}}{\partial z}+\left(\frac{\omega_{1}}{\left(1-\omega_{1}-\omega_{2}\right)^{2}}\right) \frac{\partial \omega_{2}}{\partial z}\right)\left(\left(\frac{\partial D_{21}}{\partial \omega_{1}} \frac{\partial^{2} \omega_{1}}{\partial z^{2}}+\frac{\partial \omega_{1}}{\partial z} \frac{\partial^{2} D_{21}}{\partial \omega_{1} \omega_{2}} \frac{\partial \omega_{2}}{\partial z}\right) \frac{\partial \omega_{1}}{\partial z}+\right) \\
& \left.\left.\frac{\partial \omega_{21}}{\partial \omega_{2}} \frac{\partial_{2}}{\partial z^{2}}+\frac{\partial \omega_{2}}{\partial z} \frac{\partial^{2} D_{21}}{\partial \omega_{1} \omega_{2}} \frac{\partial \omega_{1}}{\partial z}\right) \frac{\partial \omega_{2}}{\partial z}\right)+
\end{aligned}
$$$$
\left\{\begin{array}{l}
\left.\left.\left[\begin{array}{l}
\left.\left(\frac{\omega_{1}}{\left(1-\omega_{1}-\omega_{2}\right)^{2}}+\frac{1}{\left(1-\omega_{1}-\omega_{2}\right)}\right) \frac{\partial^{2} \omega_{1}}{\partial z^{2}}+\frac{\partial \omega_{1}}{\partial z}\left(\frac{2 \omega_{1}}{\left(1-\omega_{1}-\omega_{2}\right)^{3}}+\frac{1}{\left(1-\omega_{1}-\omega_{2}\right)^{2}}\right) \frac{\partial \omega_{1}}{\partial z}+\left(\frac{2 \omega_{1}}{\left(1-\omega_{1}-\omega_{2}\right)^{3}}\right) \frac{\partial \omega_{2}}{\partial z}+\right) \\
\left(\frac{1}{\left(1-\omega_{1}-\omega_{2}\right)^{2}}\right) \frac{\partial \omega_{1}}{\partial z}+\left(\frac{1}{\left(1-\omega_{1}-\omega_{2}\right)^{2}}\right) \frac{\partial \omega_{2}}{\partial z}
\end{array}\right)\right]\right\} \\
+\left[\left(\frac{\omega_{1}}{\left(1-\omega_{1}-\omega_{2}\right)^{2}}\right) \frac{\partial^{2} \omega_{2}}{\partial z^{2}}+\left(\left(\frac{2 \omega_{1}}{\left(1-\omega_{1}-\omega_{2}\right)^{3}}+\frac{1}{\left(1-\omega_{1}-\omega_{2}\right)^{2}}\right) \frac{\partial \omega_{1}}{\partial z}+\left(\frac{2 \omega_{1}}{\left(1-\omega_{1}-\omega_{2}\right)^{3}}\right) \frac{\partial \omega_{2}}{\partial z}\right)\right] \frac{\partial \omega_{2}}{\partial z}
\end{array}\right\}
$$$$
\left(\frac{\partial D_{21}}{\partial z}\right)
$$$$
\left(\left(\frac{\left(1-\omega_{2}\right)}{\left(1-\omega_{1}-\omega_{2}\right)^{2}}+\frac{\left(1-\omega_{2}\right)}{\left(1-\omega_{1}-\omega_{2}\right)^{2}}\right) \frac{\partial \omega_{1}}{\partial z}-\left(\frac{1}{\left(1-\omega_{1}-\omega_{2}\right)}\right) \frac{\partial \omega_{2}}{\partial z}\right)
$$$$
\left(\frac{\partial D_{11}}{\partial \omega_{1}} \frac{\partial^{2} \omega_{1}}{\partial z^{2}}+\frac{\partial \omega_{1}}{\partial z} \frac{\partial^{2} D_{11}}{\partial \omega_{1} \omega_{2}} \frac{\partial \omega_{2}}{\partial z}+\frac{\partial D_{11}}{\partial \omega_{2}} \frac{\partial^{2} \omega_{2}}{\partial z^{2}}+\frac{\partial \omega_{2}}{\partial z} \frac{\partial^{2} D_{11}}{\partial \omega_{1} \omega_{2}} \frac{\partial \omega_{1}}{\partial z}\right)+
$$ 


$$
\begin{aligned}
& \left(\frac{\left(1-\omega_{2}\right)}{\left(1-\omega_{1}-\omega_{2}\right)^{2}}+\frac{\left(1-\omega_{2}\right)}{\left(1-\omega_{1}-\omega_{2}\right)^{2}}\right) \frac{\partial^{2} \omega_{1}}{\partial z^{2}}+ \\
& \frac{\partial \omega_{1}}{\partial z}\left[\begin{array}{l}
\left(\frac{2\left(1-\omega_{2}\right)}{\left(1-\omega_{1}-\omega_{2}\right)^{3}}+\frac{2\left(1-\omega_{2}\right)}{\left(1-\omega_{1}-\omega_{2}\right)^{3}}\right) \frac{\partial \omega_{1}}{\partial z}+ \\
\left(\frac{2\left(1-\omega_{2}\right)}{\left(1-\omega_{1}-\omega_{2}\right)^{3}}-\frac{-1}{\left(1-\omega_{1}-\omega_{2}\right)^{2}}+\frac{2\left(1-\omega_{2}\right)}{\left(1-\omega_{1}-\omega_{2}\right)^{3}}-\frac{-1}{\left(1-\omega_{1}-\omega_{2}\right)^{2}}\right) \frac{\partial \omega_{2}}{\partial z}
\end{array}\right]- \\
& {\left[\left(\frac{1}{\left(1-\omega_{1}-\omega_{2}\right)}\right) \frac{\partial^{2} \omega_{2}}{\partial z^{2}}+\frac{\partial \omega_{2}}{\partial z}\left[\left(\frac{1}{\left(1-\omega_{1}-\omega_{2}\right)^{2}}\right) \frac{\partial \omega_{1}}{\partial z}+\left(\frac{1}{\left(1-\omega_{1}-\omega_{2}\right)^{2}}\right) \frac{\partial \omega_{2}}{\partial z}\right]\right] \frac{\partial D_{11}}{\partial z}}
\end{aligned}
$$

\section{Differentiating $f_{2}$ w.r.t $\omega_{1}$}

$$
\begin{aligned}
& \frac{\partial f_{2}}{\partial \omega_{1}}=+\left(\frac{\omega_{2}}{\left(1-\omega_{1}-\omega_{2}\right)^{2}}+\frac{1}{1-\omega_{1}-\omega_{2}}\right)\left(\frac{\partial}{\partial z}\left(D_{11} \frac{\partial \omega_{1}}{\partial z}+D_{12} \frac{\partial \omega_{2}}{\partial z}\right)\right) \frac{\partial \omega_{2}}{\partial z}+ \\
& \left(\left(\frac{2 \omega_{2}}{\left(1-\omega_{1}-\omega_{2}\right)^{3}}+\frac{1}{\left(1-\omega_{1}-\omega_{2}\right)^{2}}\right) \frac{\partial \omega_{1}}{\partial z}+\left(\frac{2 \omega_{2}}{\left(1-\omega_{1}-\omega_{2}\right)^{3}}+\frac{1}{\left(1-\omega_{1}-\omega_{2}\right)^{2}}+\right) \frac{\partial \omega_{2}}{\partial z}\right) \\
& \left(D_{11} \frac{\partial \omega_{1}}{\partial z}+D_{12} \frac{\partial \omega_{2}}{\partial z}\right) \frac{\partial \omega_{2}}{\partial z}+ \\
& \left(\frac{1-\omega_{1}}{\left(1-\omega_{1}-\omega_{2}\right)^{2}}\right)\left(\frac{\partial}{\partial z}\left(D_{22} \frac{\partial \omega_{2}}{\partial z}+D_{21} \frac{\partial \omega_{1}}{\partial z}\right)\right) \frac{\partial \omega_{2}}{\partial z}+ \\
& \left(\left(\frac{2\left(1-\omega_{1}\right)}{\left(1-\omega_{1}-\omega_{2}\right)^{3}}-\frac{1}{\left(1-\omega_{1}-\omega_{2}\right)^{2}}\right) \frac{\partial \omega_{1}}{\partial z}+\left(\frac{2\left(1-\omega_{1}\right)}{\left(1-\omega_{1}-\omega_{2}\right)^{3}}\right) \frac{\partial \omega_{2}}{\partial z}\right)\left(D_{22} \frac{\partial \omega_{2}}{\partial z}+D_{21} \frac{\partial \omega_{1}}{\partial z}\right) \\
& \frac{\partial \omega_{2}}{\partial z}+\left(\frac{\omega_{2}}{\left(1-\omega_{1}-\omega_{2}\right)^{2}}\right)\left(\frac{\partial}{\partial z}\left(D_{11} \frac{\partial \omega_{1}}{\partial z}+D_{12} \frac{\partial \omega_{2}}{\partial z}\right)\right) \frac{\partial \omega_{1}}{\partial z}+ \\
& \left(\left(\frac{2 \omega_{2}}{\left(1-\omega_{1}-\omega_{2}\right)^{3}}\right) \frac{\partial \omega_{1}}{\partial z}+\left(\frac{\omega_{2}}{\left(1-\omega_{1}-\omega_{2}\right)^{2}}+\frac{1}{1-\omega_{1}-\omega_{2}}\right) \frac{\partial \omega_{2}}{\partial z}\right)\left(D_{11} \frac{\partial \omega_{1}}{\partial z}+D_{12} \frac{\partial \omega_{2}}{\partial z}\right) \frac{\partial \omega_{1}}{\partial z}+ \\
& \left(\frac{1-\omega_{1}}{\left(1-\omega_{1}-\omega_{2}\right)^{2}}-\frac{1}{1-\omega_{1}-\omega_{2}}\right)\left(\frac{\partial}{\partial z}\left(D_{22} \frac{\partial \omega_{2}}{\partial z}+D_{21} \frac{\partial \omega_{1}}{\partial z}\right)\right) \frac{\partial \omega_{1}}{\partial z}+ \\
& \left(\left(\frac{2\left(1-\omega_{1}\right)}{\left(1-\omega_{1}-\omega_{2}\right)^{3}}-\frac{1}{\left(1-\omega_{1}-\omega_{2}\right)^{2}}+\frac{1}{\left(1-\omega_{1}-\omega_{2}\right)^{2}}\right) \frac{\partial \omega_{1}}{\partial z}+\left(\frac{2\left(1-\omega_{1}\right)}{\left(1-\omega_{1}-\omega_{2}\right)^{3}}+\frac{1}{\left(1-\omega_{1}-\omega_{2}\right)^{2}}\right) \frac{\partial \omega_{2}}{\partial z}\right) \\
& \left(D_{22} \frac{\partial \omega_{2}}{\partial z}+D_{21} \frac{\partial \omega_{1}}{\partial z}\right) \frac{\partial \omega_{1}}{\partial z}+
\end{aligned}
$$




$$
\begin{aligned}
& D_{12}\left(\frac{2 \omega_{2}}{\left(1-\omega_{1}-\omega_{2}\right)^{2}} \frac{\partial \omega_{1}}{\partial z}+\left(\frac{2 \omega_{2}}{\left(1-\omega_{1}-\omega_{2}\right)^{2}}+\frac{1}{\left(1-\omega_{1}-\omega_{2}\right)^{2}}\right) \frac{\partial \omega_{2}}{\partial z}\right)\left(\frac{\partial^{2} \omega_{2}}{\partial z^{2}}\right)+ \\
& \frac{\partial \mathrm{D}_{12}}{\partial \mathrm{z}} \frac{\omega_{2}}{\left(1-\omega_{1}-\omega_{2}\right)}\left(\frac{\partial^{2} \omega_{2}}{\partial \mathrm{z}^{2}}\right)+\frac{\left(1-\omega_{1}\right)}{\left(1-\omega_{1}-\omega_{2}\right)} \frac{\partial \mathrm{D}_{22}}{\partial \mathrm{z}}\left(\frac{\partial^{2} \omega_{2}}{\partial \mathrm{z}^{2}}\right)+ \\
& \left(\begin{array}{l}
\left(\frac{1-\omega_{1}}{\left(1-\omega_{1}-\omega_{2}\right)^{2}}-\frac{1}{1-\omega_{1}-\omega_{2}}\right) \frac{\partial \omega_{1}}{\partial z}+ \\
\frac{1-\omega_{1}}{\left(1-\omega_{1}-\omega_{2}\right)^{2}} \frac{\partial \omega_{2}}{\partial z}
\end{array}\right) D_{22}\left(\frac{\partial^{2} \omega_{2}}{\partial z^{2}}\right) \\
& +\frac{\partial D_{11}}{\partial z} \frac{\omega_{2}}{\left(1-\omega_{1}-\omega_{2}\right)}\left(\frac{\partial^{2} \omega_{1}}{\partial z^{2}}\right)+\left(\frac{\omega_{2}}{\left(1-\omega_{1}-\omega_{2}\right)^{2}} \frac{\partial \omega_{1}}{\partial z}+\left(\frac{\omega_{2}}{\left(1-\omega_{1}-\omega_{2}\right)^{2}}+\frac{1}{\left(1-\omega_{1}-\omega_{2}\right)^{2}}\right) \frac{\partial \omega_{2}}{\partial z}\right) \\
& D_{11}\left(\frac{\partial^{2} \omega_{1}}{\partial z^{2}}\right) \\
& \frac{\partial D_{12}}{\partial z} \frac{\left(1-\omega_{1}\right)}{\left(1-\omega_{1}-\omega_{2}\right)}\left(\frac{\partial^{2} \omega_{1}}{\partial z^{2}}\right)+D_{12}\left(\left(\frac{1-\omega_{1}}{\left(1-\omega_{1}-\omega_{2}\right)^{2}}-\frac{1}{1-\omega_{1}-\omega_{2}}\right) \frac{\partial \omega_{1}}{\partial z}+\frac{1-\omega_{1}}{\left(1-\omega_{1}-\omega_{2}\right)^{2}} \frac{\partial \omega_{2}}{\partial z}\right) \\
& \left(\frac{\partial^{2} \omega_{1}}{\partial z^{2}}\right)+ \\
& \frac{\omega_{2}}{\left(1-\omega_{1}-\omega_{2}\right)}\left(\frac{\partial}{\partial \omega_{1}}\left(\frac{\partial D_{11}}{\partial \omega_{2}}\right)\right)\left(\frac{\partial \omega_{1}}{\partial z} \frac{\partial \omega_{2}}{\partial z}\right)+\left(\frac{\omega_{2}}{\left(1-\omega_{1}-\omega_{2}\right)^{2}} \frac{\partial \omega_{1}}{\partial z}+\left(\frac{\omega_{2}}{\left(1-\omega_{1}-\omega_{2}\right)^{2}}+\frac{1}{\left(1-\omega_{1}-\omega_{2}\right)^{2}}\right) \frac{\partial \omega_{2}}{\partial z}\right) \\
& \left(\frac{\partial D_{11}}{\partial \omega_{2}}\right)\left(\frac{\partial \omega_{1}}{\partial z} \frac{\partial \omega_{2}}{\partial z}\right) \\
& \frac{\omega_{2}}{\left(1-\omega_{1}-\omega_{2}\right)}\left(\frac{\partial D_{12}}{\partial \omega_{1}}\right)\left(\frac{\partial \omega_{1}}{\partial z} \frac{\partial \omega_{2}}{\partial z}\right)+\frac{\omega_{2}}{\left(1-\omega_{1}-\omega_{2}\right)}\left(\frac{\partial}{\partial \omega_{1}}\left(\frac{\partial D_{12}}{\partial \omega_{1}}\right)\right)\left(\frac{\partial \omega_{1}}{\partial z} \frac{\partial \omega_{2}}{\partial z}\right)+ \\
& \left(\frac{\omega_{2}}{\left(1-\omega_{1}-\omega_{2}\right)^{2}} \frac{\partial \omega_{1}}{\partial z}+\left(\frac{\omega_{2}}{\left(1-\omega_{1}-\omega_{2}\right)^{2}}+\frac{1}{\left(1-\omega_{1}-\omega_{2}\right)^{2}}\right) \frac{\partial \omega_{2}}{\partial z}\right)\left(\frac{\partial D_{12}}{\partial \omega_{1}}\right)\left(\frac{\partial \omega_{1}}{\partial z} \frac{\partial \omega_{2}}{\partial z}\right)+ \\
& \frac{\left(1-\omega_{1}\right)}{\left(1-\omega_{1}-\omega_{2}\right)}\left(\frac{\partial}{\partial \omega_{1}}\left(\frac{\partial D_{22}}{\partial \omega_{1}}\right)\right)\left(\frac{\partial \omega_{1}}{\partial z} \frac{\partial \omega_{2}}{\partial z}\right)+\left(\begin{array}{l}
\left(\frac{1-\omega_{1}}{\left(1-\omega_{1}-\omega_{2}\right)^{2}}-\frac{1}{1-\omega_{1}-\omega_{2}}\right) \frac{\partial \omega_{1}}{\partial z}+ \\
\frac{1-\omega_{1}}{\left(1-\omega_{1}-\omega_{2}\right)^{2}} \frac{\partial \omega_{2}}{\partial z}
\end{array}\right) \\
& \left(\frac{\partial D_{22}}{\partial \omega_{1}}\right)\left(\frac{\partial \omega_{1}}{\partial z} \frac{\partial \omega_{2}}{\partial z}\right)+\frac{\left(1-\omega_{1}\right)}{\left(1-\omega_{1}-\omega_{2}\right)}\left(\frac{\partial}{\partial \omega_{1}} \frac{\partial D_{21}}{\partial \omega_{2}}\right)\left(\frac{\partial \omega_{1}}{\partial z} \frac{\partial \omega_{2}}{\partial z}\right)+
\end{aligned}
$$




$$
\begin{aligned}
& \left(\begin{array}{l}
\left(\frac{1-\omega_{1}}{\left(1-\omega_{1}-\omega_{2}\right)^{2}}-\frac{1}{1-\omega_{1}-\omega_{2}}\right) \frac{\partial \omega_{1}}{\partial z}+ \\
\frac{1-\omega_{1}}{\left(1-\omega_{1}-\omega_{2}\right)^{2}} \frac{\partial \omega_{2}}{\partial z}
\end{array}\right)\left(\frac{\partial D_{21}}{\partial \omega_{2}}\right)\left(\frac{\partial \omega_{1}}{\partial z} \frac{\partial \omega_{2}}{\partial z}\right) \frac{\omega_{2}}{\left(1-\omega_{1}-\omega_{2}\right)}\left(\frac{\partial}{\partial \omega_{1}} \frac{\partial D_{12}}{\partial \omega_{2}}\right)\left(\frac{\partial \omega_{2}}{\partial z}\right)^{2} \\
& +\left(\frac{\omega_{2}}{\left(1-\omega_{1}-\omega_{2}\right)^{2}} \frac{\partial \omega_{1}}{\partial z}+\left(\frac{\omega_{2}}{\left(1-\omega_{1}-\omega_{2}\right)^{2}}+\frac{1}{\left(1-\omega_{1}-\omega_{2}\right)^{2}}\right) \frac{\partial \omega_{2}}{\partial z}\right)\left(\frac{\partial D_{12}}{\partial \omega_{2}}\right)\left(\frac{\partial \omega_{2}}{\partial z}\right)^{2}+ \\
& \frac{\left(1-\omega_{1}\right)}{\left(1-\omega_{1}-\omega_{2}\right)}\left(\frac{\partial}{\partial \omega_{1}} \frac{\partial D_{22}}{\partial \omega_{2}}\right)\left(\frac{\partial \omega_{2}}{\partial z}\right)^{2}+\left(\begin{array}{l}
\left(\frac{1-\omega_{1}}{\left(1-\omega_{1}-\omega_{2}\right)^{2}}-\frac{1}{1-\omega_{1}-\omega_{2}}\right) \frac{\partial \omega_{1}}{\partial z}+ \\
\frac{1-\omega_{1}}{\left(1-\omega_{1}-\omega_{2}\right)^{2}} \frac{\partial \omega_{2}}{\partial z}
\end{array}\right) \\
& \left(\frac{\partial D_{22}}{\partial \omega_{2}}\right)\left(\frac{\partial \omega_{2}}{\partial z}\right)^{2}+ \\
& \frac{1-\omega_{1}}{\left(1-\omega_{1}-\omega_{2}\right)}\left(\frac{\partial}{\partial \omega_{1}} \frac{\partial D_{21}}{\partial \omega_{1}}\right)\left(\frac{\partial \omega_{1}}{\partial z}\right)^{2}\left(\left(\frac{1-\omega_{1}}{\left(1-\omega_{1}-\omega_{2}\right)^{2}}-\frac{1}{1-\omega_{1}-\omega_{2}}\right) \frac{\partial \omega_{1}}{\partial z}+\frac{1-\omega_{1}}{\left(1-\omega_{1}-\omega_{2}\right)^{2}} \frac{\partial \omega_{2}}{\partial z}\right) \\
& \left(\frac{\partial D_{21}}{\partial \omega_{1}}\right)\left(\frac{\partial \omega_{1}}{\partial z}\right)^{2}+ \\
& \frac{\omega_{2}}{\left(1-\omega_{1}-\omega_{2}\right)}\left(\frac{\partial}{\partial \omega_{1}} \frac{\partial D_{11}}{\partial \omega_{1}}\right)\left(\frac{\partial \omega_{1}}{\partial z}\right)^{2}+ \\
& \left(\frac{\omega_{2}}{\left(1-\omega_{1}-\omega_{2}\right)^{2}} \frac{\partial \omega_{1}}{\partial z}+\left(\frac{\omega_{2}}{\left(1-\omega_{1}-\omega_{2}\right)^{2}}+\frac{1}{\left(1-\omega_{1}-\omega_{2}\right)^{2}}\right) \frac{\partial \omega_{2}}{\partial z}\right)\left(\frac{\partial D_{11}}{\partial \omega_{1}}\right)\left(\frac{\partial \omega_{1}}{\partial z}\right)^{2}
\end{aligned}
$$


Differentiating $f_{2}$ w.r.t $\omega_{1 \mathrm{Z}}$

$$
\begin{aligned}
& \frac{\partial f_{2}}{\partial \omega_{1 z}}=\left(\frac{\omega_{2}}{\left(1-\omega_{1}-\omega_{2}\right)^{2}}+\frac{1}{1-\omega_{1}-\omega_{2}}\right)\left(D_{11}\right) \frac{\partial \omega_{2}}{\partial z}+\left(\frac{1-\omega_{1}}{\left(1-\omega_{1}-\omega_{2}\right)^{2}}\right)\left(D_{21}\right) \frac{\partial \omega_{2}}{\partial z}+ \\
& 2\left(\frac{\omega_{2}}{\left(1-\omega_{1}-\omega_{2}\right)^{2}}\right)\left(D_{11} \frac{\partial \omega_{1}}{\partial z}\right)+2\left(\frac{1-\omega_{1}}{\left(1-\omega_{1}-\omega_{2}\right)^{2}}-\frac{1}{1-\omega_{1}-\omega_{2}}\right)\left(D_{21} \frac{\partial \omega_{1}}{\partial z}\right)+ \\
& \frac{\omega_{2}}{\left(1-\omega_{1}-\omega_{2}\right)}\left(\frac{\partial D_{11}}{\partial \omega_{2}}\right)\left(\frac{\partial \omega_{2}}{\partial z}\right)+\frac{\omega_{2}}{\left(1-\omega_{1}-\omega_{2}\right)}\left(\frac{\partial D_{12}}{\partial \omega_{1}}\right)\left(\frac{\partial \omega_{2}}{\partial z}\right)+ \\
& \frac{\left(1-\omega_{1}\right)}{\left(1-\omega_{1}-\omega_{2}\right)}\left(\frac{\partial D_{22}}{\partial \omega_{1}}\right)\left(\frac{\partial \omega_{2}}{\partial z}\right)+\frac{\left(1-\omega_{1}\right)}{\left(1-\omega_{1}-\omega_{2}\right)}\left(\frac{\partial D_{21}}{\partial \omega_{2}}\right)\left(\frac{\partial \omega_{2}}{\partial z}\right)+ \\
& +2 \frac{1-\omega_{1}}{\left(1-\omega_{1}-\omega_{2}\right)}\left(\frac{\partial D_{21}}{\partial \omega_{1}}\right) \frac{\partial \omega_{1}}{\partial z}+2 \frac{\omega_{2}}{\left(1-\omega_{1}-\omega_{2}\right)}\left(\frac{\partial D_{11}}{\partial \omega_{1}}\right) \frac{\partial \omega_{1}}{\partial z}
\end{aligned}
$$

Differentiating $\frac{\partial f_{2}}{\partial \omega_{1 z}}$ w.r.t $z$, we get

$\frac{\partial}{\partial z}\left(\frac{\partial f_{2}}{\partial \omega_{1 z}}\right)=\left(\frac{\omega_{2}}{\left(1-\omega_{1}-\omega_{2}\right)^{2}}+\frac{1}{1-\omega_{1}-\omega_{2}}\right)\left(D_{11}\right) \frac{\partial^{2} \omega_{2}}{\partial z^{2}}+\left(\frac{\omega_{2}}{\left(1-\omega_{1}-\omega_{2}\right)^{2}}+\frac{1}{1-\omega_{1}-\omega_{2}}\right)\left(\frac{\partial D_{11}}{\partial z}\right) \frac{\partial \omega_{2}}{\partial z}+$

$\left(\left(\frac{2 \omega_{2}}{\left(1-\omega_{1}-\omega_{2}\right)^{3}}+\frac{1}{\left(1-\omega_{1}-\omega_{2}\right)^{2}}\right) \frac{\partial \omega_{1}}{\partial z}+\left(\frac{2 \omega_{2}}{\left(1-\omega_{1}-\omega_{2}\right)^{3}}+\frac{1}{\left(1-\omega_{1}-\omega_{2}\right)^{2}}+\frac{1}{\left(1-\omega_{1}-\omega_{2}\right)^{2}}\right) \frac{\partial \omega_{2}}{\partial z}\right) D_{11} \frac{\partial \omega_{2}}{\partial z}+$

$\left(\frac{1-\omega_{1}}{\left(1-\omega_{1}-\omega_{2}\right)^{2}}\right)\left(D_{21}\right) \frac{\partial^{2} \omega_{2}}{\partial z^{2}}+\left(\frac{1-\omega_{1}}{\left(1-\omega_{1}-\omega_{2}\right)^{2}}\right)\left(\frac{\partial D_{21}}{\partial z}\right) \frac{\partial \omega_{2}}{\partial z}+\left(\left(\frac{2\left(1-\omega_{1}\right)}{\left(1-\omega_{1}-\omega_{2}\right)^{3}}-\frac{1}{\left(1-\omega_{1}-\omega_{2}\right)^{2}}\right) \frac{\partial \omega_{1}}{\partial z}+\frac{2\left(1-\omega_{1}\right)}{\left(1-\omega_{1}-\omega_{2}\right)^{3}} \frac{\partial \omega_{2}}{\partial z}\right)\left(D_{21}\right) \frac{\partial \omega_{2}}{\partial z}$

$2\left(\frac{\omega_{2}}{\left(1-\omega_{1}-\omega_{2}\right)^{2}}\right) D_{11}\left(\frac{\partial^{2} \omega_{1}}{\partial z^{2}}\right)+2\left(\frac{\omega_{2}}{\left(1-\omega_{1}-\omega_{2}\right)^{2}}\right)\left(\frac{\partial D_{11}}{\partial z}\right)\left(\frac{\partial \omega_{1}}{\partial z}\right)+2\left(\frac{2 \omega_{2}}{\left(1-\omega_{1}-\omega_{2}\right)^{3}} \frac{\partial \omega_{1}}{\partial z}+\left(\frac{2 \omega_{2}}{\left(1-\omega_{1}-\omega_{2}\right)^{3}}+\frac{1}{\left(1-\omega_{1}-\omega_{2}\right)^{2}}\right) \frac{\partial \omega_{2}}{\partial z}\right) D_{11}\left(\frac{\partial \omega_{1}}{\partial z}\right)$

$2\left(\frac{1-\omega_{1}}{\left(1-\omega_{1}-\omega_{2}\right)^{2}}-\frac{1}{1-\omega_{1}-\omega_{2}}\right) D_{21}\left(\frac{\partial^{2} \omega_{1}}{\partial z^{2}}\right)+2\left(\frac{1-\omega_{1}}{\left(1-\omega_{1}-\omega_{2}\right)^{2}}-\frac{1}{1-\omega_{1}-\omega_{2}}\right)\left(\frac{\partial D_{21}}{\partial z}\right)\left(\frac{\partial \omega_{1}}{\partial z}\right)+$

$2\left(\left(\frac{2\left(1-\omega_{1}\right)}{\left(1-\omega_{1}-\omega_{2}\right)^{3}}-\frac{1}{\left(1-\omega_{1}-\omega_{2}\right)^{2}}+\frac{1}{\left(1-\omega_{1}-\omega_{2}\right)^{2}}\right)\left(\frac{\partial \omega_{1}}{\partial z}\right)+\left(\frac{2\left(1-\omega_{1}\right)}{\left(1-\omega_{1}-\omega_{2}\right)^{3}}+\frac{1}{\left(1-\omega_{1}-\omega_{2}\right)^{2}}\right) \frac{\partial \omega_{2}}{\partial z}\right) D_{21}\left(\frac{\partial \omega_{1}}{\partial z}\right)+$ 


$$
\begin{aligned}
& \frac{\omega_{2}}{\left(1-\omega_{1}-\omega_{2}\right)}\left(\frac{\partial D_{11}}{\partial \omega_{2}}\right)\left(\frac{\partial^{2} \omega_{2}}{\partial z^{2}}\right)+\frac{\omega_{2}}{\left(1-\omega_{1}-\omega_{2}\right)}\left(\frac{\partial}{\partial z} \frac{\partial D_{11}}{\partial \omega_{2}}\right)\left(\frac{\partial \omega_{2}}{\partial z}\right)+ \\
& \left(\frac{\omega_{2}}{\left(1-\omega_{1}-\omega_{2}\right)^{2}} \frac{\partial \omega_{1}}{\partial z}+\left(\frac{\omega_{2}}{\left(1-\omega_{1}-\omega_{2}\right)^{2}}+\frac{1}{\left(1-\omega_{1}-\omega_{2}\right)^{2}}\right) \frac{\partial \omega_{2}}{\partial z}\right)\left(\frac{\partial D_{11}}{\partial \omega_{2}}\right)\left(\frac{\partial \omega_{2}}{\partial z}\right) \\
& \frac{\omega_{2}}{\left(1-\omega_{1}-\omega_{2}\right)}\left(\frac{\partial D_{12}}{\partial \omega_{1}}\right)\left(\frac{\partial^{2} \omega_{2}}{\partial z^{2}}\right)+\frac{\omega_{2}}{\left(1-\omega_{1}-\omega_{2}\right)}\left(\frac{\partial}{\partial z} \frac{\partial D_{12}}{\partial \omega_{1}}\right)\left(\frac{\partial \omega_{2}}{\partial z}\right)+ \\
& \left(\frac{\omega_{2}}{\left(1-\omega_{1}-\omega_{2}\right)^{2}} \frac{\partial \omega_{1}}{\partial z}+\left(\frac{\omega_{2}}{\left(1-\omega_{1}-\omega_{2}\right)^{2}}+\frac{1}{\left(1-\omega_{1}-\omega_{2}\right)^{2}}\right) \frac{\partial \omega_{2}}{\partial z}\right)\left(\frac{\partial D_{12}}{\partial \omega_{1}}\right)\left(\frac{\partial \omega_{2}}{\partial z}\right) \\
& \frac{\left(1-\omega_{1}\right)}{\left(1-\omega_{1}-\omega_{2}\right)}\left(\frac{\partial D_{22}}{\partial \omega_{1}}\right)\left(\frac{\partial^{2} \omega_{2}}{\partial z^{2}}\right)+\frac{\left(1-\omega_{1}\right)}{\left(1-\omega_{1}-\omega_{2}\right)}\left(\frac{\partial}{\partial z} \frac{\partial D_{22}}{\partial \omega_{1}}\right)\left(\frac{\partial \omega_{2}}{\partial z}\right)+ \\
& \left(\left(\frac{1-\omega_{1}}{\left(1-\omega_{1}-\omega_{2}\right)^{2}}-\frac{1}{1-\omega_{1}-\omega_{2}}\right) \frac{\partial \omega_{1}}{\partial z}+\frac{1-\omega_{1}}{\left(1-\omega_{1}-\omega_{2}\right)^{2}} \frac{\partial \omega_{2}}{\partial z}\right)\left(\frac{\partial D_{22}}{\partial \omega_{1}}\right)\left(\frac{\partial \omega_{2}}{\partial z}\right) \\
& \frac{\left(1-\omega_{1}\right)}{\left(1-\omega_{1}-\omega_{2}\right)}\left(\frac{\partial D_{21}}{\partial \omega_{2}}\right)\left(\frac{\partial^{2} \omega_{2}}{\partial z}\right)+\frac{\left(1-\omega_{1}\right)}{\left(1-\omega_{1}-\omega_{2}\right)}\left(\frac{\partial}{\partial z} \frac{\partial D_{21}}{\partial \omega_{2}}\right)\left(\frac{\partial \omega_{2}}{\partial z}\right)+ \\
& \left(\left(\frac{1-\omega_{1}}{\left(1-\omega_{1}-\omega_{2}\right)^{2}}-\frac{1}{1-\omega_{1}-\omega_{2}}\right) \frac{\partial \omega_{1}}{\partial z}+\frac{1-\omega_{1}}{\left(1-\omega_{1}-\omega_{2}\right)^{2}} \frac{\partial \omega_{2}}{\partial z}\right)\left(\frac{\partial D_{21}}{\partial \omega_{2}}\right)\left(\frac{\partial \omega_{2}}{\partial z}\right)+ \\
& 2 \frac{1-\omega_{1}}{\left(1-\omega_{1}-\omega_{2}\right)}\left(\frac{\partial D_{21}}{\partial \omega_{1}}\right) \frac{\partial^{2} \omega_{1}}{\partial z^{2}}+2 \frac{1-\omega_{1}}{\left(1-\omega_{1}-\omega_{2}\right)}\left(\frac{\partial}{\partial z} \frac{\partial D_{21}}{\partial \omega_{1}}\right) \frac{\partial \omega_{1}}{\partial z}+ \\
& 2\left(\left(\frac{1-\omega_{1}}{\left(1-\omega_{1}-\omega_{2}\right)^{2}}-\frac{1}{1-\omega_{1}-\omega_{2}}\right) \frac{\partial \omega_{1}}{\partial z}+\frac{1-\omega_{1}}{\left(1-\omega_{1}-\omega_{2}\right)^{2}} \frac{\partial \omega_{2}}{\partial z}\right)\left(\frac{\partial D_{21}}{\partial \omega_{1}}\right) \frac{\partial \omega_{1}}{\partial z} \\
& 2 \frac{\omega_{2}}{\left(1-\omega_{1}-\omega_{2}\right)}\left(\frac{\partial D_{11}}{\partial \omega_{1}}\right) \frac{\partial^{2} \omega_{1}}{\partial z^{2}}+2 \frac{\omega_{2}}{\left(1-\omega_{1}-\omega_{2}\right)}\left(\frac{\partial}{\partial z} \frac{\partial D_{11}}{\partial \omega_{1}}\right) \frac{\partial \omega_{1}}{\partial z}+ \\
& \left(\frac{\omega_{2}}{\left(1-\omega_{1}-\omega_{2}\right)^{2}} \frac{\partial \omega_{1}}{\partial z}+\left(\frac{\omega_{2}}{\left(1-\omega_{1}-\omega_{2}\right)^{2}}+\frac{1}{\left(1-\omega_{1}-\omega_{2}\right)^{2}}\right) \frac{\partial \omega_{2}}{\partial z}\right)\left(\frac{\partial D_{11}}{\partial \omega_{1}}\right) \frac{\partial \omega_{1}}{\partial z}
\end{aligned}
$$

Differentiating of $f_{2}$ w.r.t $\omega_{1 z z}$

$$
\frac{\partial f_{2}}{\partial \omega_{1 z z}}=D_{11} \frac{\omega_{2}}{\left(1-\omega_{1}-\omega_{2}\right)}+D_{12} \frac{\left(1-\omega_{1}\right)}{\left(1-\omega_{1}-\omega_{2}\right)}
$$

Taking second derivative of $\frac{\partial f_{2}}{\partial \omega_{1 z z}}$ w.r.t $z$ 


$$
\begin{aligned}
& \frac{\partial}{\partial z}\left(\frac{\partial f_{2}}{\partial \omega_{1 z z}}\right)=\left(\frac{\omega_{2}}{\left(1-\omega_{1}-\omega_{2}\right)^{2}} \frac{\partial \omega_{1}}{\partial z}+\left(\frac{\omega_{2}}{\left(1-\omega_{1}-\omega_{2}\right)^{2}}+\frac{1}{\left(1-\omega_{1}-\omega_{2}\right)^{2}}\right) \frac{\partial \omega_{2}}{\partial z}\right) D_{11}+ \\
& \left(\frac{\partial D_{11}}{\partial z}\right) \frac{\omega_{2}}{\left(1-\omega_{1}-\omega_{2}\right)}+ \\
& \left(\left(\frac{1-\omega_{1}}{\left(1-\omega_{1}-\omega_{2}\right)^{2}}-\frac{1}{1-\omega_{1}-\omega_{2}}\right) \frac{\partial \omega_{1}}{\partial z}+\frac{1-\omega_{1}}{\left(1-\omega_{1}-\omega_{2}\right)^{2}} \frac{\partial \omega_{2}}{\partial z}\right) D_{12}+\left(\frac{\partial D_{12}}{\partial z}\right) \frac{\left(1-\omega_{1}\right)}{\left(1-\omega_{1}-\omega_{2}\right)} \\
& \frac{\partial}{\partial z}\left(\frac{\partial f_{2}}{\partial \omega_{1 z z}}\right)=\frac{\omega_{2}}{\left(1-\omega_{1}-\omega_{2}\right)^{2}} \frac{\partial \omega_{1}}{\partial z} D_{11}+\left(\frac{\omega_{2}}{\left(1-\omega_{1}-\omega_{2}\right)^{2}}+\frac{1}{\left(1-\omega_{1}-\omega_{2}\right)^{2}}\right) \frac{\partial \omega_{2}}{\partial z} D_{11}+ \\
& \left(\frac{\partial D_{11}}{\partial z}\right) \frac{\omega_{2}}{\left(1-\omega_{1}-\omega_{2}\right)}+ \\
& \left(\frac{1-\omega_{1}}{\left(1-\omega_{1}-\omega_{2}\right)^{2}}-\frac{1}{1-\omega_{1}-\omega_{2}}\right) \frac{\partial \omega_{1}}{\partial z} D_{12}+\frac{1-\omega_{1}}{\left(1-\omega_{1}-\omega_{2}\right)^{2}} \frac{\partial \omega_{2}}{\partial z} D_{12}+\left(\frac{\partial D_{12}}{\partial z}\right) \frac{\left(1-\omega_{1}\right)}{\left(1-\omega_{1}-\omega_{2}\right)}
\end{aligned}
$$

Taking third derivative of $\frac{\partial f_{2}}{\partial \omega_{1 z z}}$ w.r.t z

$$
\begin{aligned}
& \frac{\partial^{2}}{\partial z^{2}}\left(\frac{\partial f_{2}}{\partial \omega_{1 z z}}\right)=\frac{\omega_{2}}{\left(1-\omega_{1}-\omega_{2}\right)^{2}} \frac{\partial \omega_{1}}{\partial z} \frac{\partial D_{11}}{\partial z}+\frac{\omega_{2}}{\left(1-\omega_{1}-\omega_{2}\right)^{2}} \frac{\partial^{2} \omega_{1}}{\partial z^{2}} D_{11}+ \\
& \left(\frac{2 \omega_{2}}{\left(1-\omega_{1}-\omega_{2}\right)^{3}} \frac{\partial \omega_{1}}{\partial z}+\left(\frac{2 \omega_{2}}{\left(1-\omega_{1}-\omega_{2}\right)^{3}}+\frac{1}{\left(1-\omega_{1}-\omega_{2}\right)^{2}} \frac{\partial \omega_{2}}{\partial z}\right) \frac{\partial \omega_{1}}{\partial z} D_{11}+\right. \\
& \left(\frac{\omega_{2}}{\left(1-\omega_{1}-\omega_{2}\right)^{2}}+\frac{1}{\left(1-\omega_{1}-\omega_{2}\right)^{2}}\right) \frac{\partial \omega_{2}}{\partial z} \frac{\partial D_{11}}{\partial z}+\left(\frac{\omega_{2}}{\left(1-\omega_{1}-\omega_{2}\right)^{2}}+\frac{1}{\left(1-\omega_{1}-\omega_{2}\right)^{2}}\right) \frac{\partial^{2} \omega_{2}}{\partial z^{2}} D_{11}+ \\
& \left(\left(\frac{2 \omega_{2}}{\left(1-\omega_{1}-\omega_{2}\right)^{3}}+\frac{2}{\left(1-\omega_{1}-\omega_{2}\right)^{3}}\right) \frac{\partial \omega_{1}}{\partial z}+\left(\frac{2 \omega_{2}}{\left(1-\omega_{1}-\omega_{2}\right)^{3}}+\frac{1}{\left(1-\omega_{1}-\omega_{2}\right)^{2}}+\frac{2}{\left(1-\omega_{1}-\omega_{2}\right)^{3}}\right) \frac{\partial \omega_{2}}{\partial z}\right) \frac{\partial \omega_{2}}{\partial z} D_{11}+ \\
& \left(\frac{\partial^{2} D_{11}}{\partial z^{2}}\right) \frac{\omega_{2}}{\left(1-\omega_{1}-\omega_{2}\right)}+\left(\frac{\partial D_{11}}{\partial z}\right)\left(\frac{\omega_{2}}{\left(1-\omega_{1}-\omega_{2}\right)^{2}} \frac{\partial \omega_{1}}{\partial z}+\left(\frac{\omega_{2}}{\left(1-\omega_{1}-\omega_{2}\right)^{2}}+\frac{1}{\left(1-\omega_{1}-\omega_{2}\right)^{2}}\right) \frac{\partial \omega_{2}}{\partial z}\right)+ \\
& \left(\frac{1-\omega_{1}}{\left(1-\omega_{1}-\omega_{2}\right)^{2}}-\frac{1}{1-\omega_{1}-\omega_{2}}\right) \frac{\partial \omega_{1}}{\partial z}\left(\frac{\partial D_{12}}{\partial z}\right)+\left(\frac{1-\omega_{1}}{\left(1-\omega_{1}-\omega_{2}\right)^{2}}-\frac{1}{1-\omega_{1}-\omega_{2}}\right) \frac{\partial^{2} \omega_{1}}{\partial z^{2}} D_{12}+ \\
& +\left(\left(\frac{2\left(1-\omega_{1}\right)}{\left(1-\omega_{1}-\omega_{2}\right)^{3}}-\frac{1}{1-\omega_{1}-\omega_{2}}-\frac{1}{\left(1-\omega_{1}-\omega_{2}\right)^{2}}\right) \frac{\partial \omega_{1}}{\partial z}+\left(\frac{2\left(1-\omega_{1}\right)}{\left(1-\omega_{1}-\omega_{2}\right)^{3}}-\frac{1}{\left(1-\omega_{1}-\omega_{2}\right)^{2}}\right) \frac{\partial \omega_{2}}{\partial z}\right) \frac{\partial \omega_{1}}{\partial z} D_{12}
\end{aligned}
$$




$$
\begin{aligned}
& +\frac{1-\omega_{1}}{\left(1-\omega_{1}-\omega_{2}\right)^{2}} \frac{\partial \omega_{2}}{\partial z}\left(\frac{\partial D_{12}}{\partial z}\right)+\frac{1-\omega_{1}}{\left(1-\omega_{1}-\omega_{2}\right)^{2}} \frac{\partial^{2} \omega_{2}}{\partial z^{2}} D_{12}+\left(\begin{array}{l}
\left(\frac{2\left(1-\omega_{1}\right)}{\left(1-\omega_{1}-\omega_{2}\right)^{3}}-\frac{1}{\left(1-\omega_{1}-\omega_{2}\right)^{2}}\right) \frac{\partial \omega_{1}}{\partial z}+ \\
\left(\frac{2\left(1-\omega_{1}\right)}{\left(1-\omega_{1}-\omega_{2}\right)^{3}}\right) \frac{\partial \omega_{2}}{\partial z}
\end{array}\right) \frac{\partial \omega_{2}}{\partial z} D_{12} \\
& \left(\frac{\partial^{2} D_{12}}{\partial z^{2}}\right) \frac{\left(1-\omega_{1}\right)}{\left(1-\omega_{1}-\omega_{2}\right)}+\left(\left(\frac{1-\omega_{1}}{\left(1-\omega_{1}-\omega_{2}\right)^{2}}-\frac{1}{\left(1-\omega_{1}-\omega_{2}\right)}\right) \frac{\partial \omega_{1}}{\partial z}+\left(\frac{1-\omega_{1}}{\left(1-\omega_{1}-\omega_{2}\right)^{2}}\right) \frac{\partial \omega_{2}}{\partial z}\right)\left(\frac{\partial D_{12}}{\partial z}\right)
\end{aligned}
$$

The variational derivatives of $f_{1}$ and $f_{2}$, Equations (4.51-4.54) are given below

$-\lambda_{1} \frac{\partial f_{1}}{\partial D_{11}} \delta D_{11}=-\lambda_{1}\left[\begin{array}{l}\frac{1-\omega_{2}}{\left(1-\omega_{1}-\omega_{2}\right)^{2}}\left(\frac{\partial \omega_{1}}{\partial z}\right)^{2}+\left(\frac{1-\omega_{2}}{\left(1-\omega_{1}-\omega_{2}\right)^{2}}-\frac{1}{1-\omega_{1}-\omega_{2}}\right)\left(\frac{\partial \omega_{1}}{\partial z} \frac{\partial \omega_{2}}{\partial z}\right)+{ }_{\frac{\left(1-\omega_{2}\right)}{\left(1-\omega_{1}-\omega_{2}\right)}}\left(\frac{\partial^{2} \omega_{1}}{\partial z^{2}}\right)\end{array}\right] \delta D_{11}$

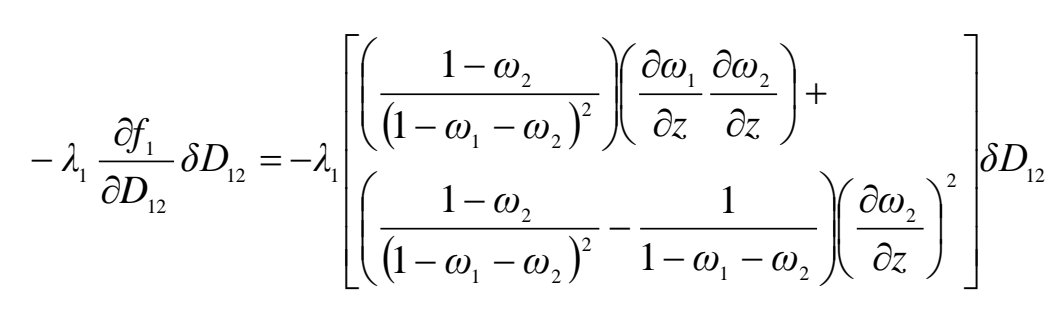

$$
-\lambda_{1} \frac{\partial f_{1}}{\partial D_{21}} \delta D_{21}=-\lambda_{1}\left[\begin{array}{l}
\left(\frac{\omega_{1}}{\left(1-\omega_{1}-\omega_{2}\right)^{2}}+\frac{1}{1-\omega_{1}-\omega_{2}}\right)\left(\frac{\partial \omega_{1}}{\partial z}\right)^{2}+ \\
\left(\frac{\omega_{1}}{\left(1-\omega_{1}-\omega_{2}\right)^{2}}\right)\left(\frac{\partial \omega_{2}}{\partial z} \frac{\partial \omega_{1}}{\partial z}\right)+ \\
\frac{\omega_{1}}{\left(1-\omega_{1}-\omega_{2}\right)}\left(\frac{\partial^{2} \omega_{1}}{\partial z^{2}}\right)+\frac{\left(1-\omega_{2}\right)}{\left(1-\omega_{1}-\omega_{2}\right)}\left(\frac{\partial^{2} \omega_{2}}{\partial z^{2}}\right)
\end{array}\right] \delta D_{21}
$$


$-\lambda_{1} \frac{\partial f_{1}}{\partial D_{22}} \delta D_{22}=-\lambda_{1}\left[\begin{array}{l}\left.\left(\frac{\omega_{1}}{\left(1-\omega_{1}-\omega_{2}\right)^{2}}+\frac{1}{1-\omega_{1}-\omega_{2}}\right)\left(\frac{\partial \omega_{1}}{\partial z} \frac{\partial \omega_{2}}{\partial z}\right)+\right] \\ \left(\frac{\omega_{1}}{\left(1-\omega_{1}-\omega_{2}\right)^{2}}\right)\left(\frac{\partial \omega_{2}}{\partial z}\right)^{2}+ \\ \left(1-\omega_{1}-\omega_{2}\right)\end{array}\right] \delta D_{22}$

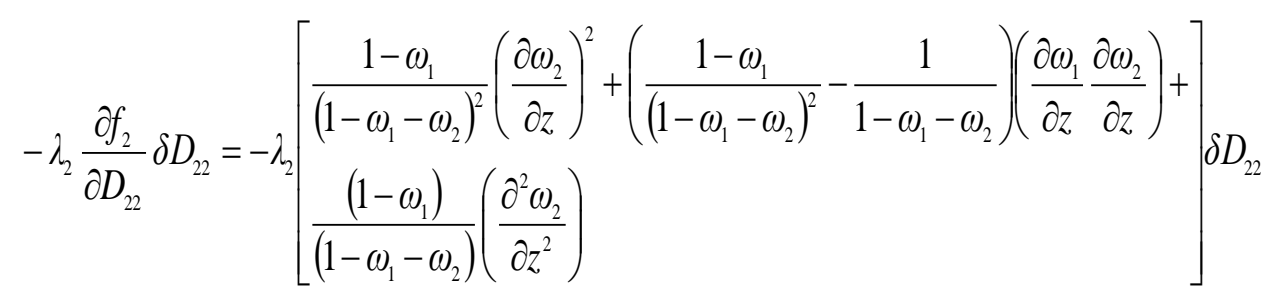

(4.75)

$-\lambda_{2} \frac{\partial f_{2}}{\partial D_{21}} \delta D_{21}=-\lambda_{2}\left[\begin{array}{l}\left(\frac{1-\omega_{1}}{\left(1-\omega_{1}-\omega_{2}\right)^{2}}\right)\left(\frac{\partial \omega_{1}}{\partial z} \frac{\partial \omega_{2}}{\partial z}\right)+ \\ \left(\frac{1-\omega_{1}}{\left(1-\omega_{1}-\omega_{2}\right)^{2}}-\frac{1}{1-\omega_{1}-\omega_{2}}\right)\left(\frac{\partial \omega_{1}}{\partial z}\right)^{2}\end{array}\right] \delta D_{21}$

$$
-\lambda_{2} \frac{\partial f_{2}}{\partial D_{12}} \delta D_{12}=-\lambda_{2}\left[\begin{array}{l}
\left(\frac{\omega_{2}}{\left(1-\omega_{1}-\omega_{2}\right)^{2}}+\frac{1}{1-\omega_{1}-\omega_{2}}\right)\left(\frac{\partial \omega_{2}}{\partial z}\right)^{2}+ \\
\left(\frac{\omega_{2}}{\left(1-\omega_{1}-\omega_{2}\right)^{2}}\right)\left(\frac{\partial \omega_{2}}{\partial z} \frac{\partial \omega_{1}}{\partial z}\right)+ \\
\frac{\omega_{2}}{\left(1-\omega_{1}-\omega_{2}\right)}\left(\frac{\partial^{2} \omega_{2}}{\partial z^{2}}\right)+\frac{\left(1-\omega_{1}\right)}{\left(1-\omega_{1}-\omega_{2}\right)}\left(\frac{\partial^{2} \omega_{1}}{\partial z^{2}}\right)
\end{array}\right] \delta D_{12}
$$




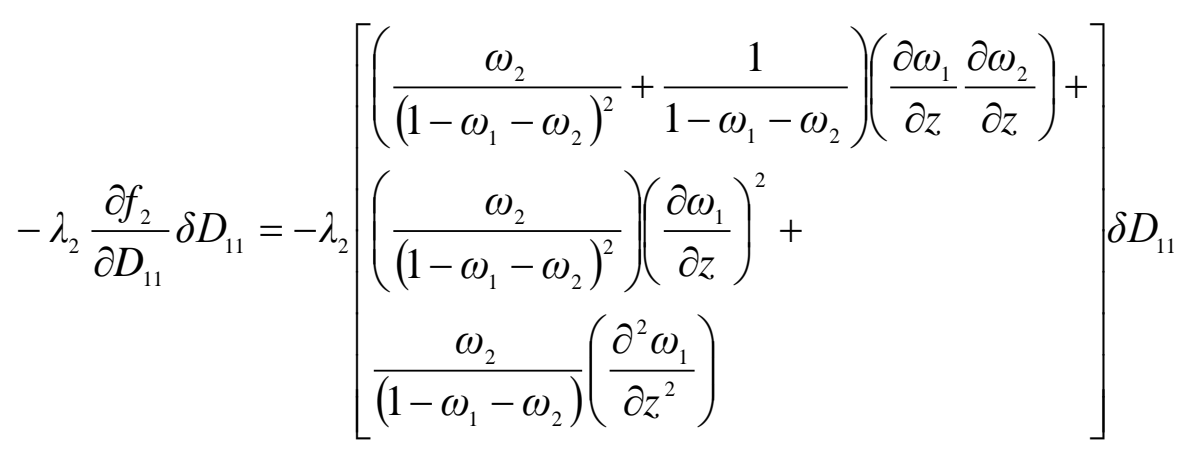

\subsection{Computational Algorithm}

Based on the necessary conditions for the minimum of the objective functional, the following computational algorithm was developed to determine the concentrationdependent, multi-component diffusion coefficients.

1 Step A: Initialize $D_{11}, D_{12}, D_{21}$ and $D_{22}$. Four initial estimates of diffusivities, i.e. $D_{11}, D_{12}, D_{21}$ and $D_{22}$, were provided to initialize the program.

2 Step B: Simultaneously integrate the state equations (model) subject to the initial and boundary conditions, to obtain the values of $\omega_{1}$ or 2 for each node. To implement the numerical solution, the state equations were expressed in finite-difference form along $z$ direction. The model of the diffusion cell was divided into equal-spaced grid points in the $z$ direction. In this way, each state equation was transformed into a set of simultaneous ordinary differential equations. The fifth-order, adaptive-step method of Runge-Kutta- 
Fehlberg was used with Cash-Karp parameters to achieve an accurate solution with minimum computational effort. The values of state variables $\left(D_{i j}\right.$ and $\left.\omega_{i j}\right), i, j=1,2$ at each grid point in the space of $t$ and $z$ are saved.

3 Step D: Evaluate the objective functional.

4 If there is no difference between the calculated mass and the experimental mass of the gases absorbed then STOP, otherwise go the next step.

5 Step E: Integrate the costate equations backward, subject to the final boundary conditions, using stored values of $\omega_{i j}(z, t)$ to get the values of $\lambda{ }_{i, j}(z, t)$ at each grid point. The costate equations must be solved backward in the time domain. Since they are dependent on concentration, they can be only solved after solving the state equation in the forward direction in the time domain. The solution of these equations provides the variational derivatives to be used in the next step.

6 Step F: Improve $D_{i, j}\left(\omega_{1}, \omega_{2}\right)$ using the gradient correction.

7 Obtain the new and improved values of $D_{i, j}\left(\omega_{1}, \omega_{2}\right)$ at each grid point in the time interval using the variational derivative as follows:

$\mathrm{D}_{\mathrm{int}}^{\text {new }}=\omega_{\text {int }}+\delta \mathrm{D}_{\text {int }}$ where $\mathrm{D}_{\mathrm{int}}=-\varepsilon \frac{\partial \mathrm{J}}{\partial \mathrm{D}_{\mathrm{int}}}$ and $\varepsilon$ is a small positive number whose optimal value provides maximum reduction in the objective functional.

Using $D_{\text {int }}^{\text {new }}$, repeat the computations Step 2 onward until there is no further improvement in the objective functional. When the improvement is negligible, the values are optimal. 
The variational derivatives were used in conjunction with the Broyden-Fletcher-GoldfarbShanno algorithm (BFGS) method to improve the diffusivities. The optimal control algorithm was programmed in C++. Figure 4.2 shows the computational algorithm.

The architecture of the computer program that was used to estimate optimal values of concentration dependent multi-component diffusivities is shown in Figure 4.3. The computer program is divided in five main Blocks, namely: A, B, C, D and E. We will discuss these Blocks briefly. Block A includes the main file of the program that consists of BroydenFletcher-Goldfarb-Shanno algorithm (BFGS) and model class. The BFGS is gradient improvement method. Model class solves the mathematical model. Model class is connected to the Block B that contains the mass transfer model, experimental results and the input file that contains various information incluing diffusivity guesses, number of grid points, step size, tolerance, molecular weight of the polymer, polymer density etc.

When the program executes, the model class solves the differential equations i.e. state equations (in forwards direction) in Block B using fifth-order adaptive-step method of Runge-Kutta-Fehlberg with Cash-Karp parameters method and the information in the input file. Once the differential equations are solved, the programme calculates the difference between the calculated gas mass absorbed and the experimental gas mass absorbed in Block C using composite Simpson $1 / 3$ and Simpson 1/8 integration rule.

Next, the program solves the costate equations (in backward direction), in Block B, using Runge-Kutta-Fehlberg fifth order method and the results obtained by solving the state equation previously. 


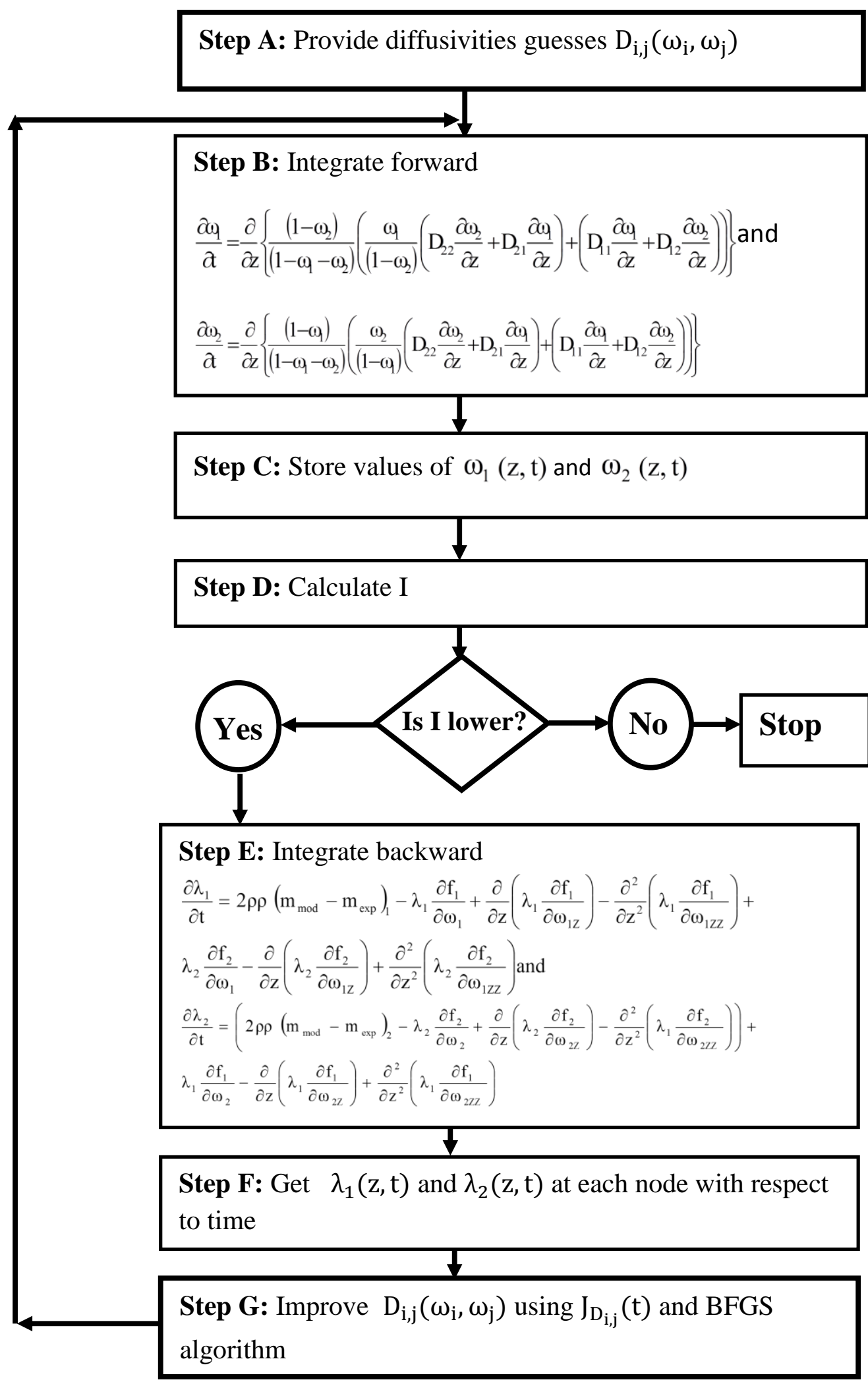

Figure 4.2. Methodology for determining diffusivities 


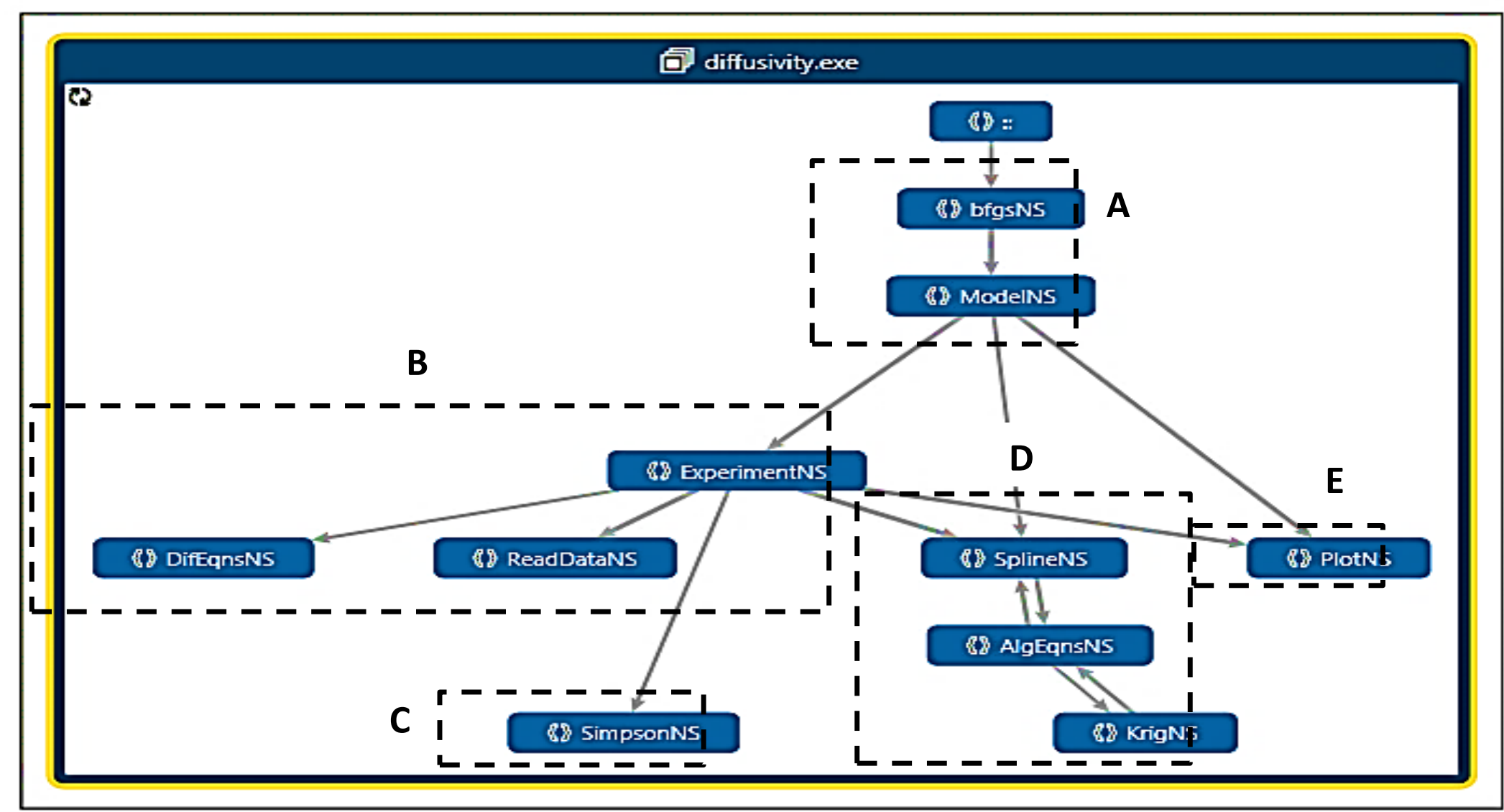

Figure 4.3 Architecture of the computer program 
Based on the difference between the calculated gas mass absorbed and the experimental gas mass absorbed the program makes the use of variational derivatives and modify the diffusivity estimate using Krig in Block D. Krig is a 3D interpolation method.

The BFGS uses all the above information via model class and minimizes the difference between the calculated gas mass absorbed and the experimental gas mass absorbed. The results are printed in Block $\mathrm{E}$.

The above process continues until difference between the calculated gas mass absorbed and the experimental gas mass absorbed is minimum. The $\mathrm{C}++$ codes for calculating variational derivatives and the improvement in the diffusivity values are given in appendix C. The results obtained in this study are presented in the next chapter. 


\section{RESULTS and DISCUSSION}

Since this work is a first step towards developing an optimal control framework to determine concentration dependent multi-component diffusivities so we decided to work at room temperature $\left(23^{\circ} \mathrm{C}\right)$ and a pressure of $224 \mathrm{kPa}$. Using the aforementioned experiments and calculations, we determined the concentration-dependent multicomponent diffusivities and solubility of nitrogen and carbon dioxide in polystyrene. The algorithm developed in Chapter 3 was used to determine the concentration-dependent, multi-component diffusivities. The parameters used in the calculations are provided in Table 5.1

Table 5.1 Parameters used in calculating diffusivities

\begin{tabular}{ll}
\hline Parameters & Value \\
\hline Mass of polymer & $5 \times 10^{-3} \mathrm{~kg}$ \\
\hline Density of the polymer & $1060 \mathrm{~kg} / \mathrm{m}^{3}$ \\
\hline Diameter of the polymer sample & $4 \times 10^{-2} \mathrm{~m}$ \\
\hline Thickness of the polymer sample & $5.211 \times 10^{-3} \mathrm{~m}$ \\
\hline Number of D vs. $\boldsymbol{\omega}$ points & 10 \\
\hline $\begin{array}{l}\text { Number of grid points along the } z \\
\text { direction }\end{array}$ & 10 and 15 \\
\hline
\end{tabular}

To arrive at the optimal diffusivities we provided different diffusivities guesses, the computer program was run and the improvement in the objective functional, and the concentration dependant diffusivities obtained were recorded. Approximately, hundreds of 
trials were run with different diffusivities guesses and only few selected that have shown good improvement in the objective functional are shown in Table 5.2. The dimension of the diffusivities in Table 5.2 is $\mathrm{m}^{2} / \mathrm{s}$. The best improvement is seen in trial 117 and was considered to be the final result. Table 5.2 shows that by increasing the number of grid points the improvement in the objective function was reduced.

Table 5.2 Various trials for obtaining optimal diffusivities.

\begin{tabular}{|c|c|c|c|c|c|c|c|}
\hline Trial & $\begin{array}{l}D_{11} \\
\times 10^{8}\end{array}$ & $\begin{array}{l}\mathrm{D}_{12} \\
\times 10^{8}\end{array}$ & $\begin{array}{l}\mathrm{D}_{21} \\
\times 10^{9}\end{array}$ & $\begin{array}{l}\mathrm{D}_{22} \\
\times 10^{8}\end{array}$ & $\begin{array}{l}\text { Grid } \\
\text { Points }\end{array}$ & Iteration & $\begin{array}{l}\text { Objective } \\
\text { functional J }\end{array}$ \\
\hline 1 & 14 & 14 & 3.3 & 33 & 10 & 317 & 0.14980 \\
\hline 2 & 1.4 & 0.14 & 3.3 & 3.3 & 10 & 120 & 0.13466 \\
\hline 3 & 1.4 & 1.4 & 3.3 & 3.3 & 10 & 64 & 0.13411 \\
\hline 4 & 1.6 & 1.4 & 3.3 & 3.6 & 10 & 11 & 0.13346 \\
\hline 5 & 3.6 & 1.4 & 3.3 & 3.9 & 10 & 51 & 0.13161 \\
\hline 6 & 4.2 & 3.5 & 4.3 & 5.0 & 10 & 88 & 0.12628 \\
\hline 7 & 5.2 & 3.5 & 4.3 & 7.0 & 10 & 123 & 0.12036 \\
\hline 8 & 6.2 & 3.5 & 4.3 & 8.0 & 10 & 24 & 0.12002 \\
\hline 9 & 7.2 & 4.5 & 5.3 & 9.0 & 10 & 32 & 0.11748 \\
\hline 10 & 8.2 & 0.45 & 43 & 9.0 & 10 & 57 & 0.11577 \\
\hline 11 & 9.5 & 0.55 & 58 & 9.0 & 10 & 48 & 0.11532 \\
\hline 12 & 9.5 & 0.95 & 58 & 9.0 & 10 & 14 & 0.11526 \\
\hline 13 & 9.0 & 9.5 & 61 & 9.0 & 10 & 295 & 0.10409 \\
\hline 14 & 9.1 & 0.95 & 81 & 9.2 & 10 & 130 & 0.11000 \\
\hline 15 & 9.1 & 0.95 & 8.1 & 9.2 & 10 & 165 & 0.11422 \\
\hline
\end{tabular}




\begin{tabular}{|c|c|c|c|c|c|c|c|}
\hline 16 & 11 & 0.95 & 8.1 & 12 & 10 & 255 & 0.13743 \\
\hline 17 & 11 & 0.95 & 81 & 12 & 10 & 46 & 0.13847 \\
\hline 18 & 11 & 9.5 & 81 & 12 & 10 & 32 & 0.13328 \\
\hline 19 & 9.1 & 9.5 & 91 & 9.2 & 10 & 61 & 0.13353 \\
\hline 20 & 21 & 9.5 & 91 & 9.2 & 10 & 240 & 0.13173 \\
\hline 21 & 21 & 7.5 & 91 & 72 & 10 & 97 & 0.13573 \\
\hline 22 & 31 & 7.5 & 21 & 22 & 10 & 212 & 0.12775 \\
\hline 23 & 22 & 9.5 & 95 & 15 & 10 & 199 & 0.34413 \\
\hline 24 & 32 & 9.5 & 95 & 35 & 10 & 40 & 0.33314 \\
\hline 25 & 52 & 9.5 & 95 & 55 & 10 & 16 & 0.32460 \\
\hline 26 & 72 & 9.5 & 95 & 75 & 10 & 25 & 0.31954 \\
\hline 27 & 82 & 9.5 & 95 & 85 & 10 & 16 & 0.31760 \\
\hline 28 & 82 & 9.9 & 99 & 85 & 10 & 25 & 0.31734 \\
\hline 29 & 82 & 10 & 100 & 85 & 10 & 15 & 0.31747 \\
\hline 30 & 82 & 17 & 170 & 85 & 10 & 15 & 0.31747 \\
\hline 31 & 86 & 27 & 270 & 86 & 10 & 17 & 0.31621 \\
\hline 32 & 89 & 28 & 280 & 89 & 10 & 11 & 0.31445 \\
\hline 33 & 91 & 28 & 280 & 91 & 10 & 35 & 0.31384 \\
\hline 34 & 91 & 59 & 590 & 91 & 10 & 25 & 0.31341 \\
\hline 35 & 91 & 65 & 590 & 85 & 10 & 12 & 0.30985 \\
\hline 36 & 96 & 59 & 590 & 95 & 10 & 11 & 0.31009 \\
\hline 37 & 97 & 60 & 590 & 96 & 10 & 18 & 0.30995 \\
\hline 38 & 57 & 10 & 1000 & 92 & 10 & 26 & 0.31585 \\
\hline 39 & 75 & 58 & 1000 & 85 & 10 & 22 & 0.31087 \\
\hline 40 & 75 & 58 & 1000 & 100 & 10 & 21 & 0.30966 \\
\hline 41 & 75 & 58 & 1000 & 700 & 10 & 39 & 0.31009 \\
\hline
\end{tabular}




\begin{tabular}{|c|c|c|c|c|c|c|c|}
\hline 42 & 10 & 1 & 10 & 60 & 10 & 26 & 0.11350 \\
\hline 43 & 0.15 & 0.15 & 1.5 & 0.15 & 10 & 56 & 1.43297 \\
\hline 44 & 0.17 & 0.17 & 1.7 & 0.17 & 10 & 53 & 1.42830 \\
\hline 45 & 0.19 & 0.19 & 1.9 & 0.19 & 10 & 50 & 1.42304 \\
\hline 46 & 0.23 & 0.23 & 2.3 & 0.23 & 10 & 41 & 1.41158 \\
\hline 47 & 0.35 & 0.35 & 3.5 & 0.35 & 10 & 56 & 1.37661 \\
\hline 48 & 0.37 & 0.37 & 3.7 & 0.37 & 10 & 65 & 1.37104 \\
\hline 49 & 0.3 & 0.3 & 3 & 0.3 & 10 & 47 & 1.39092 \\
\hline 50 & 0.4 & 0.4 & 4 & 0.4 & 10 & 73 & 1.36286 \\
\hline 51 & 0.1 & 0.4 & 4 & 0.63 & 10 & 13 & 0.83190 \\
\hline 52 & 1 & 0.4 & 4 & 8.3 & 10 & 17 & 1.23294 \\
\hline 53 & 3.3 & 0.4 & 4 & 0.13 & 10 & 8 & 0.92094 \\
\hline 54 & 0.33 & 0.4 & 4 & 0.93 & 10 & 73 & 1.36286 \\
\hline 55 & 0.43 & 0.4 & 4 & 0.83 & 10 & 50 & 1.42304 \\
\hline 56 & 0.53 & 0.4 & 4 & 1 & 10 & 73 & 1.36286 \\
\hline 57 & 0.83 & 0.4 & 4 & 3.3 & 10 & 50 & 1.42304 \\
\hline 58 & 9.3 & 0.4 & 4 & 0.63 & 10 & 56 & 1.37661 \\
\hline 59 & 14 & 14 & 3.3 & 33 & 15 & 288 & 0.15513 \\
\hline 60 & 1.4 & 0.14 & 3.3 & 3.3 & 15 & 102 & 0.13510 \\
\hline 61 & 1.4 & 1.4 & 3.3 & 3.3 & 15 & 52 & 0.10141 \\
\hline 62 & 1.6 & 1.4 & 3.3 & 3.6 & 15 & 6 & 0.13106 \\
\hline 63 & 3.6 & 1.4 & 3.3 & 3.9 & 15 & 44 & 0.13214 \\
\hline 64 & 4.2 & 3.5 & 4.3 & 5.0 & 15 & 79 & 0.13117 \\
\hline 65 & 5.2 & 3.5 & 4.3 & 7.0 & 15 & 111 & 0.13112 \\
\hline 66 & 6.2 & 3.5 & 4.3 & 8.0 & 15 & 13 & 0.12115 \\
\hline 67 & 7.2 & 4.5 & 5.3 & 9.0 & 15 & 27 & 0.11887 \\
\hline
\end{tabular}




\begin{tabular}{|c|c|c|c|c|c|c|c|}
\hline 68 & 8.2 & 0.45 & 43 & 9.0 & 15 & 49 & 0.11775 \\
\hline 69 & 9.5 & 0.55 & 58 & 9.0 & 15 & 38 & 0.11721 \\
\hline 70 & 9.5 & 0.95 & 58 & 9.0 & 15 & 5 & 0.11511 \\
\hline 71 & 9.0 & 9.5 & 61 & 9.0 & 15 & 246 & 0.11005 \\
\hline 72 & 9.1 & 0.95 & 81 & 9.2 & 15 & 119 & 0.11105 \\
\hline 73 & 9.1 & 0.95 & 8.1 & 9.2 & 15 & 135 & 0.11511 \\
\hline 74 & 11 & 0.95 & 8.1 & 12 & 15 & 210 & 0.13925 \\
\hline 75 & 11 & 0.95 & 81 & 12 & 15 & 39 & 0.13889 \\
\hline 76 & 11 & 9.5 & 81 & 12 & 15 & 27 & 0.13420 \\
\hline 77 & 9.1 & 9.5 & 91 & 9.2 & 15 & 54 & 0.13567 \\
\hline 78 & 21 & 9.5 & 91 & 9.2 & 15 & 223 & 0.13281 \\
\hline 79 & 21 & 7.5 & 91 & 72 & 15 & 81 & 0.13866 \\
\hline 80 & 31 & 7.5 & 21 & 22 & 15 & 198 & 0.12952 \\
\hline 81 & 22 & 9.5 & 95 & 15 & 15 & 154 & 0.34622 \\
\hline 82 & 32 & 9.5 & 95 & 35 & 15 & 29 & 0.34257 \\
\hline 83 & 52 & 9.5 & 95 & 55 & 15 & 11 & 0.32510 \\
\hline 84 & 72 & 9.5 & 95 & 75 & 15 & 16 & 0.32311 \\
\hline 85 & 82 & 9.5 & 95 & 85 & 15 & 11 & 0.31822 \\
\hline 86 & 82 & 9.9 & 99 & 85 & 15 & 14 & 0.31981 \\
\hline 87 & 82 & 10 & 100 & 85 & 15 & 11 & 0.31810 \\
\hline 88 & 82 & 17 & 170 & 85 & 15 & 12 & 0.31793 \\
\hline 89 & 86 & 27 & 270 & 86 & 15 & 10 & 0.31699 \\
\hline 90 & 89 & 28 & 280 & 89 & 15 & 6 & 0.31552 \\
\hline 91 & 91 & 28 & 280 & 91 & 15 & 26 & 0.31441 \\
\hline 92 & 91 & 59 & 590 & 91 & 15 & 14 & 0.31436 \\
\hline 93 & 91 & 65 & 590 & 85 & 15 & 8 & 0.31012 \\
\hline
\end{tabular}




\begin{tabular}{|c|c|c|c|c|c|c|c|}
\hline 94 & 96 & 59 & 590 & 95 & 15 & 7 & 0.31102 \\
\hline 95 & 97 & 60 & 590 & 96 & 15 & 11 & 0.31132 \\
\hline 96 & 57 & 10 & 1000 & 92 & 15 & 17 & 0.31864 \\
\hline 97 & 75 & 58 & 1000 & 85 & 15 & 13 & 0.31783 \\
\hline 98 & 75 & 58 & 1000 & 100 & 15 & 14 & 0.31102 \\
\hline 99 & 75 & 58 & 1000 & 700 & 15 & 27 & 0.32038 \\
\hline 100 & 10 & 1 & 10 & 60 & 15 & 18 & 0.11822 \\
\hline 101 & 0.15 & 0.15 & 1.5 & 0.15 & 15 & 50 & 1.44213 \\
\hline 102 & 0.17 & 0.17 & 1.7 & 0.17 & 15 & 44 & 1.44578 \\
\hline 103 & 0.19 & 0.19 & 1.9 & 0.19 & 15 & 45 & 1.44785 \\
\hline 104 & 0.23 & 0.23 & 2.3 & 0.23 & 15 & 35 & 1.42314 \\
\hline 105 & 0.35 & 0.35 & 3.5 & 0.35 & 15 & 50 & 1.39746 \\
\hline 106 & 0.37 & 0.37 & 3.7 & 0.37 & 15 & 54 & 1.38012 \\
\hline 107 & 0.3 & 0.3 & 3 & 0.3 & 15 & 40 & 1.40121 \\
\hline 108 & 0.4 & 0.4 & 4 & 0.4 & 15 & 65 & 1.38874 \\
\hline 109 & 0.1 & 0.4 & 4 & 0.63 & 15 & 9 & 0.85510 \\
\hline 110 & 1 & 0.4 & 4 & 8.3 & 15 & 9 & 1.24402 \\
\hline 111 & 3.3 & 0.4 & 4 & 0.13 & 15 & 3 & 0.92101 \\
\hline 112 & 0.33 & 0.4 & 4 & 0.93 & 15 & 59 & 1.38231 \\
\hline 113 & 0.43 & 0.4 & 4 & 0.83 & 15 & 38 & 1.42412 \\
\hline 114 & 0.53 & 0.4 & 4 & 1 & 15 & 66 & 1.37621 \\
\hline 115 & 0.83 & 0.4 & 4 & 3.3 & 15 & 38 & 1.46701 \\
\hline 116 & 9.3 & 0.4 & 4 & 0.63 & 15 & 48 & 1.38823 \\
\hline 117 & 15 & 30 & 13 & 23 & 15 & 290 & 0.00071 \\
\hline
\end{tabular}


Figure 5.1 shows the convergence of the functional optimization yielding the optimal diffusivity matrix $D_{11}, D_{12}, D_{21}$, and $D_{22}$. In trial 117 , by the iterative refinement of the initial estimates of the diffusivities, the value of J decreased monotonically from $1.55 \times 10^{-3}$ to a low value of $7.17 \times 10^{-4}$, that is, at the optimum where the gradient correction of the mass transfer model tended to zero and further improvement was insignificant. At the optimum where the gradient correction of the mass transfer model is zero there is an excellent agreement between the experimental and optimal calculated values of the nitrogen and carbon dioxide in the polystyrene. Figures 5.2 and 5.3 shows this agreement between the experimental and optimal calculated values of masses of two gases in the polymer.

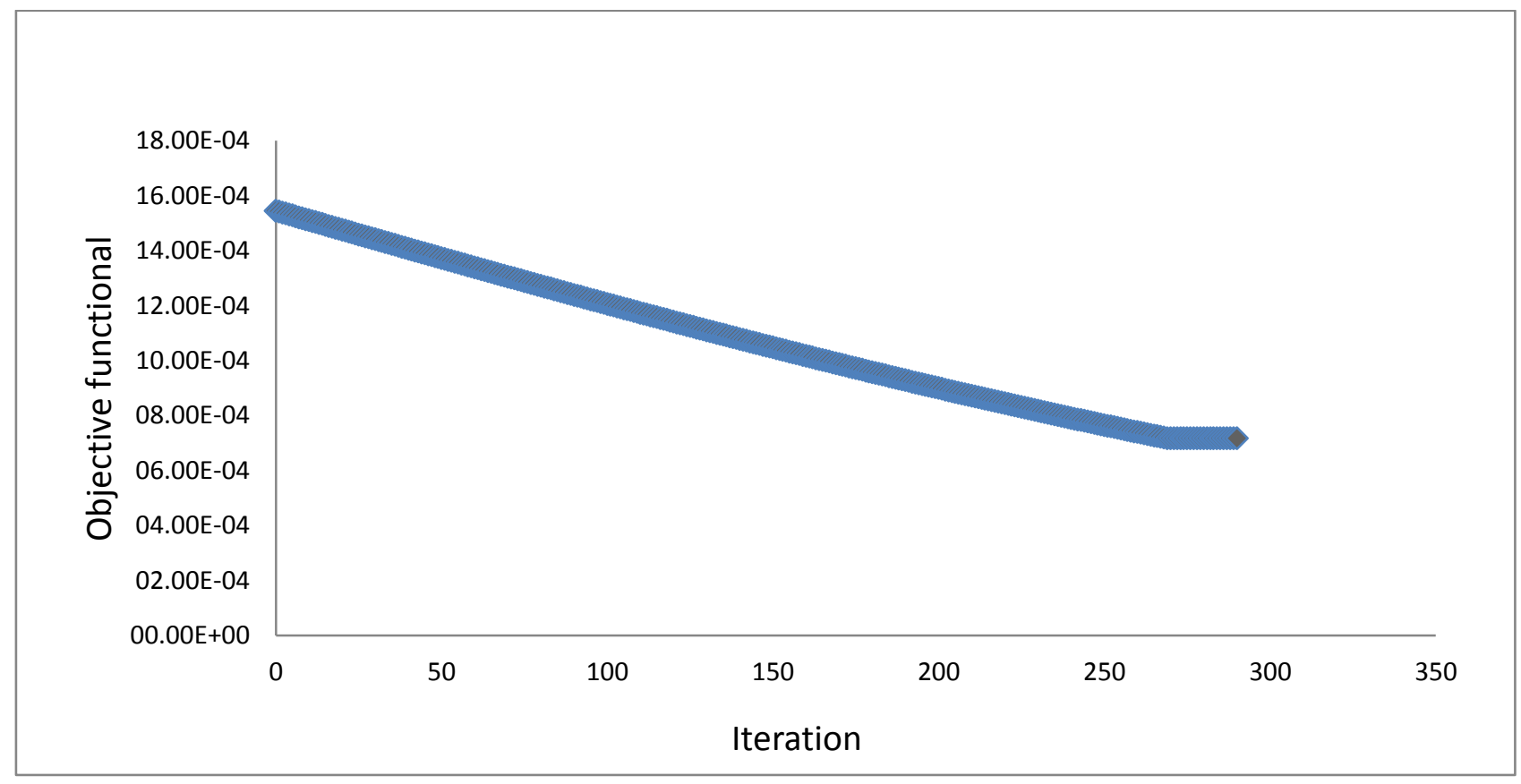

Figure 5.1 Convergence of objective functional 


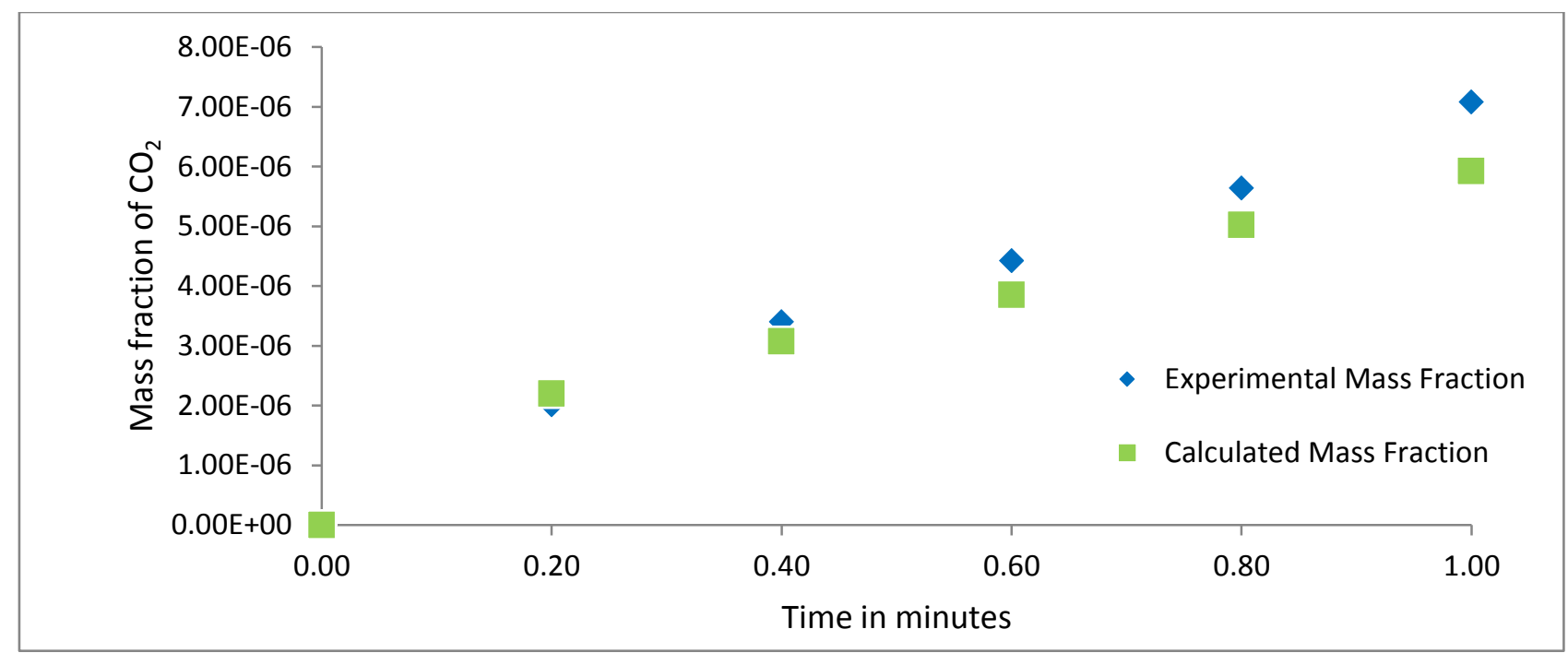

Figure 5.2 Experimental and calculated mass of carbon dioxide absorbed in polystyrene.

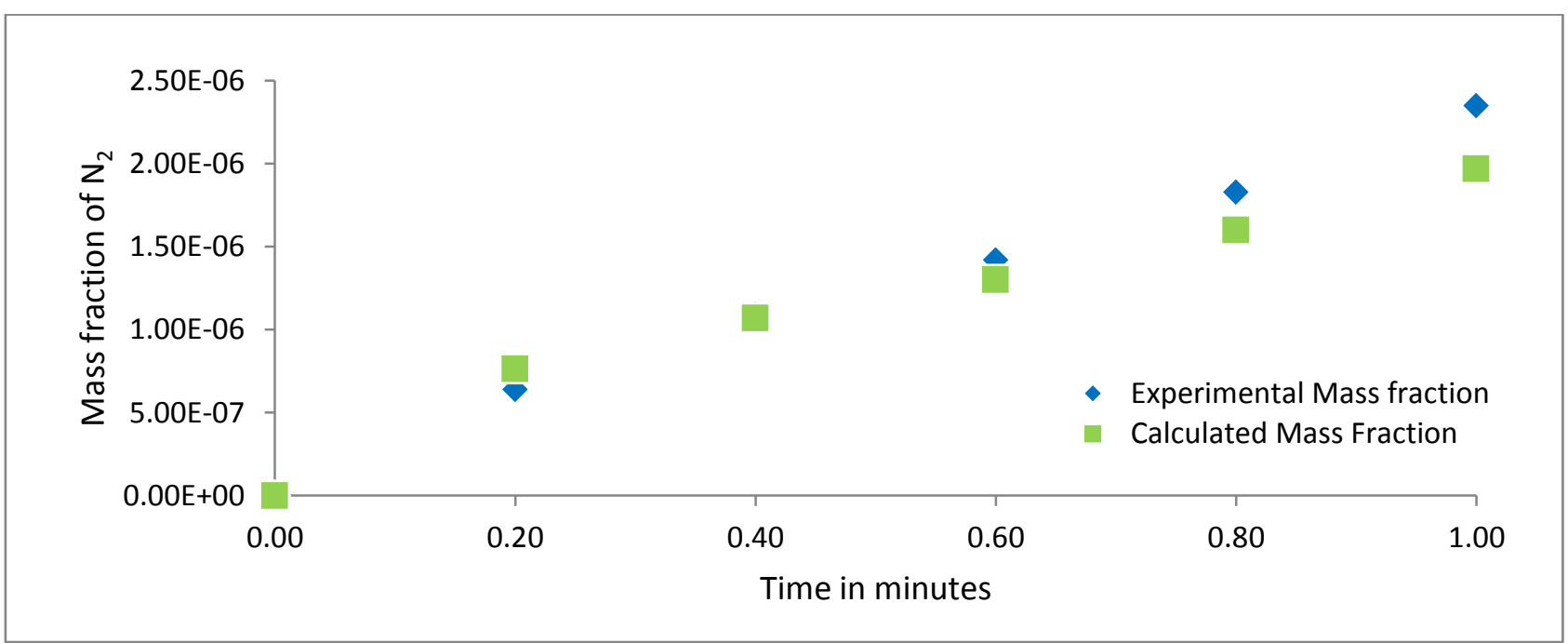

Figure 5.3. Experimental and calculated mass of nitrogen absorbed in polystyrene

During the diffusion experiments, the diffusion of carbon dioxide was found to be more compared to the diffusion of nitrogen. This is in accordance with the previous findings 
where optimal control technique was used and carbon dioxide is found to have higher diffusion compared to nitrogen (Kundra et al., 2011). Figures 5.4 and 5.5 show the interfacial mass concentration of nitrogen and carbon dioxide versus time. Figure 5.4 and 5.5 shows as the diffusion process progresses, the mass fraction of carbon dioxide reduces gradually at the surface of the polystyrene due to more diffusion of carbon dioxide compared to nitrogen. On the other hand, the amount of nitrogen increases gradually at the surface of polystyrene due to the less diffusion of nitrogen compared to carbon dioxide.

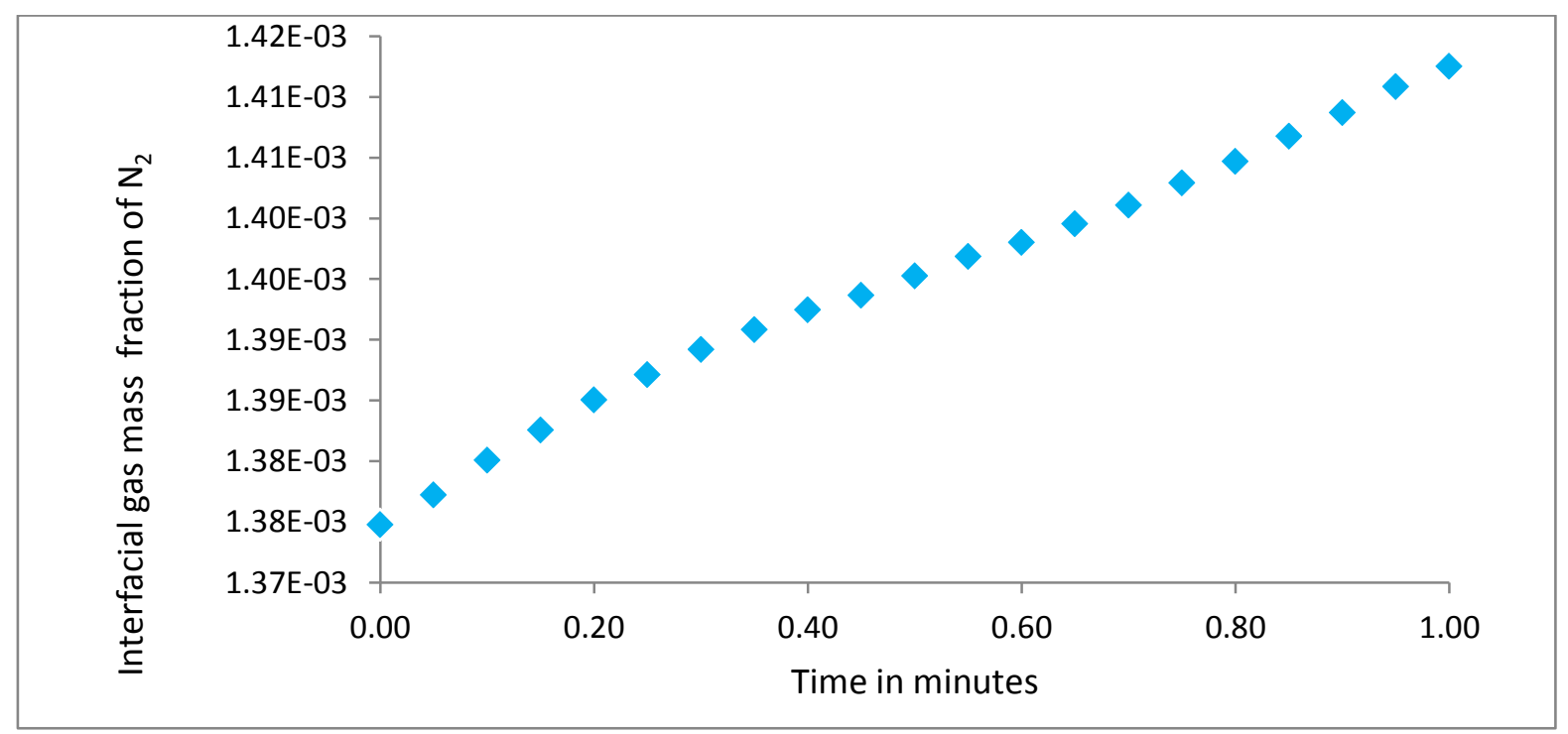

Figure 5.4. Interfacial mass fraction of nitrogen 


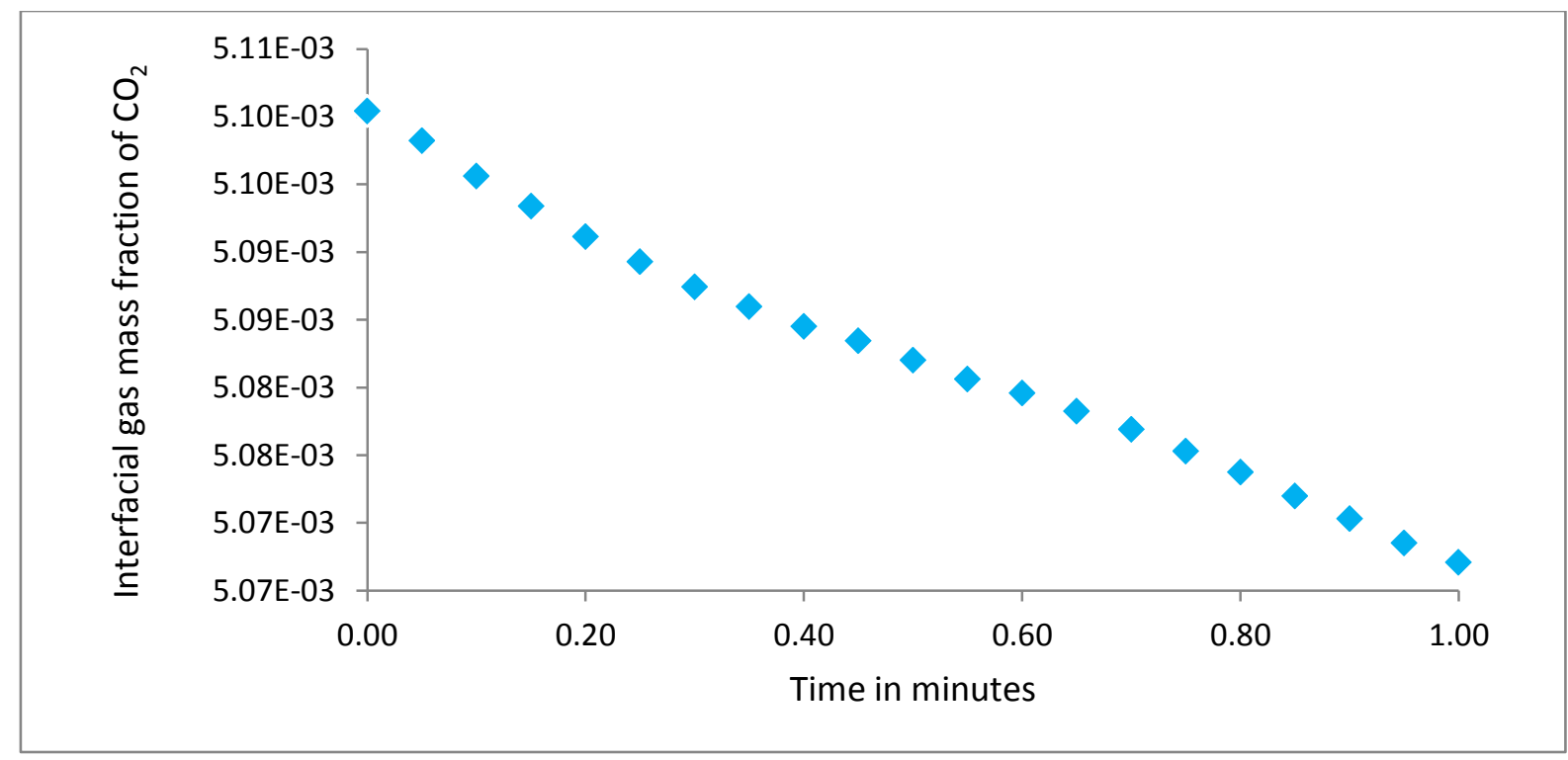

Figure 5.5. Interfacial mass fraction of carbon dioxide

Figures 5.6 through 5.9 show the experimentally-determined, composition-dependent diffusivities of nitrogen and carbon dioxide in polystyrene.

Figure 5.6 shows the changes observed in $D_{11}$. The main diffusion coefficient $D_{11}$, was observed to be increased slightly with the increasing concentration of carbon dioxide. The highest diffusivity $2.22 \times 10^{-8} \mathrm{~m}^{2} / \mathrm{s}$ for $\mathrm{D}_{11}$, which was obtained for an nitrogen mass fraction of $3.14 \times 10^{-4}$ and a carbon dioxide mass fraction of $5.67 \times 10^{-4}$. Diffusivity $\mathrm{D}_{11}$ decreased gradually as the concentration of nitrogen and carbon dioxide increased. A higher range of diffusivity values were obtained for mass fractions in the range of 0 to $5 \times 10^{-4}$ for carbon dioxide and in the range of 0 to $1.41 \times 10^{-3}$ for nitrogen. The diffusivity values varied in the range of $10^{-8}$ to $10^{-9} \mathrm{~m}^{2} \mathrm{~s}^{-1}$. From Figure 5.6 , we can say that the multi-component diffusion coefficients are a unimodal function of mass fractions of carbon dioxide in the range of 0 to $5 \times 10^{-4}$ and of mass fractions of nitrogen in the 
range of 0 to $1.41 \times 10^{-3}$, i.e., the values of the diffusion coefficients increase and reach maximum values as the concentrations of the gases increase. Interestingly, the values decrease from the maximum as the concentration of carbon dioxide continues to increase. Similar results have been reported in various solvent-polymer systems (J. L. Duda et al., 1978; Jitendra et al., 2009; Kundra et al., 2011; Vrentas, et al., 1982).

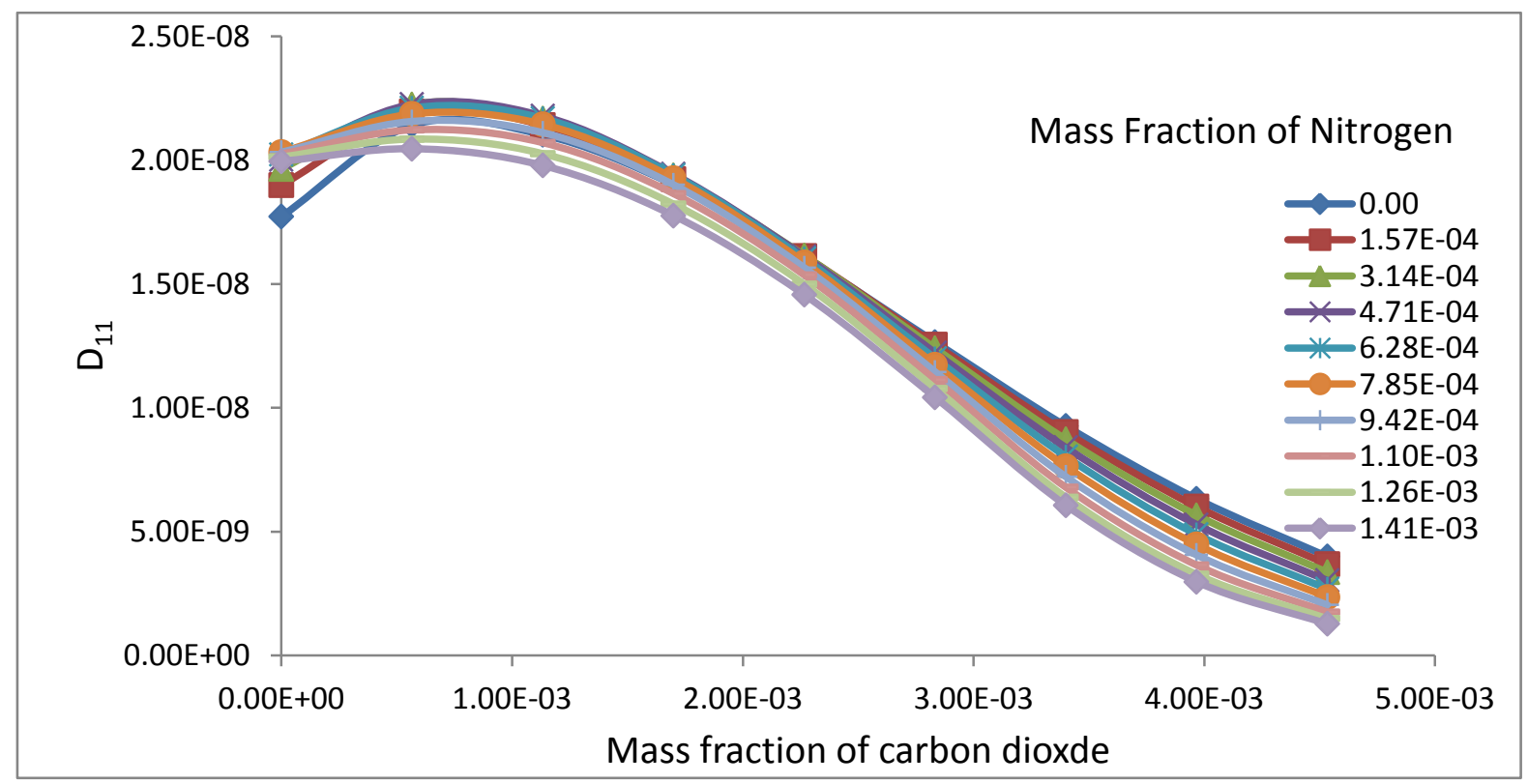

Figure 5.6. Main diffusion coefficient, $D_{11}$, of nitrogen in polystyrene

Figure 5.7 shows the result of cross-diffusion coefficient $D_{12}$. The cross-diffusion coefficient, $D_{12}$ increases as the concentration of nitrogen and carbon dioxide increases. Cross diffusion coefficient $D_{12}$ represents the diffusion of nitrogen due to the concentration gradient of carbon dioxide. The value of $D_{12}$ is found to be lower than that of $D_{21}$ that means the diffusion of carbon dioxide due to nitrogen is less compared to the 
diffusion of nitrogen due to carbon dioxide. This could be due to the fact that carbon dioxide is a bigger molecule than nitrogen so the effect of intermolecular force of attraction of nitrogen on carbon dioxide is high. The cross-diffusion coefficient $D_{12}$ was in the order of $10^{-9} \mathrm{~m}^{2} \mathrm{~s}^{-1}$

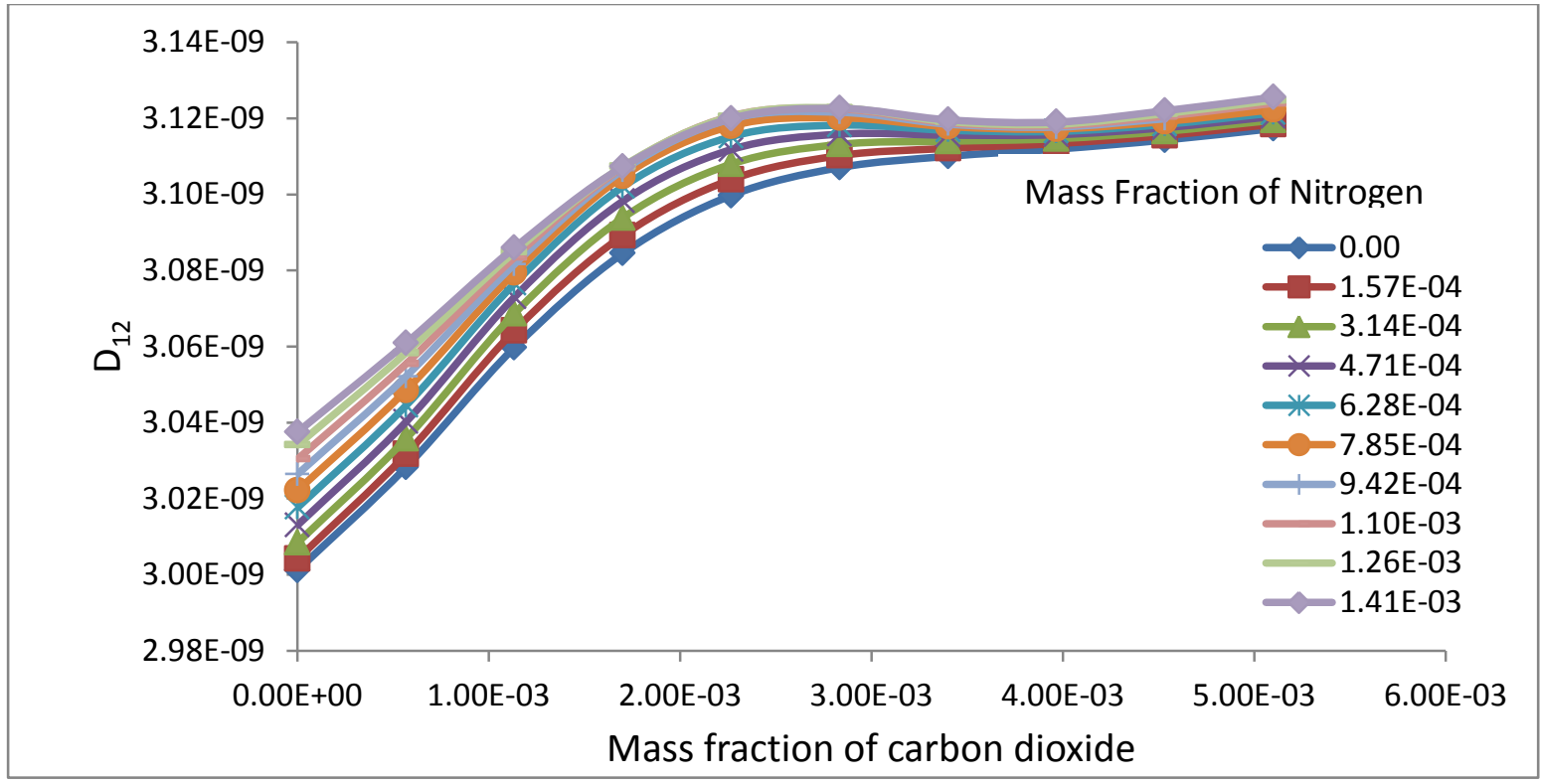

Figure 5.7. Cross-diffusion coefficient, $D_{12}$, of nitrogen in polystyrene

Figure 5.8 shows the result of cross diffusion coefficient $D_{21}$. The diffusivity of carbon dioxide increased slightly as the mass fraction of carbon dioxide increased from 0 to $1.70 \times 10^{-3}$. After the mass fraction of carbon dioxide increased beyond $1.70 \times 10^{-3}$, the diffusivity decreased, reaching a minimum when the mass fraction of carbon dioxide was $5.10 \times 10^{-3}$. The diffusivity values were on the order $10^{-7} \mathrm{~m}^{2} \mathrm{~s}^{-1}$. Cross diffusion coefficient $D_{21}$ represents the diffusion of carbon dioxide due to the concentration gradient of nitrogen. The initial increase in the $D_{21}$ values could be due to higher diffusion of nitrogen 
due to the concentration gradient of carbon dioxide. Since there is lots of empty spaces available in the polymer matrix initially, the diffusivity value of $D_{21}$ is higher. Once the space in the polymer matrix is saturated with the two gases the effect of nitrogen on carbon dioxide decreases resulting in the lower values of $D_{21}$ (Jitendra et al., 2009; Kundra et al., 2011).

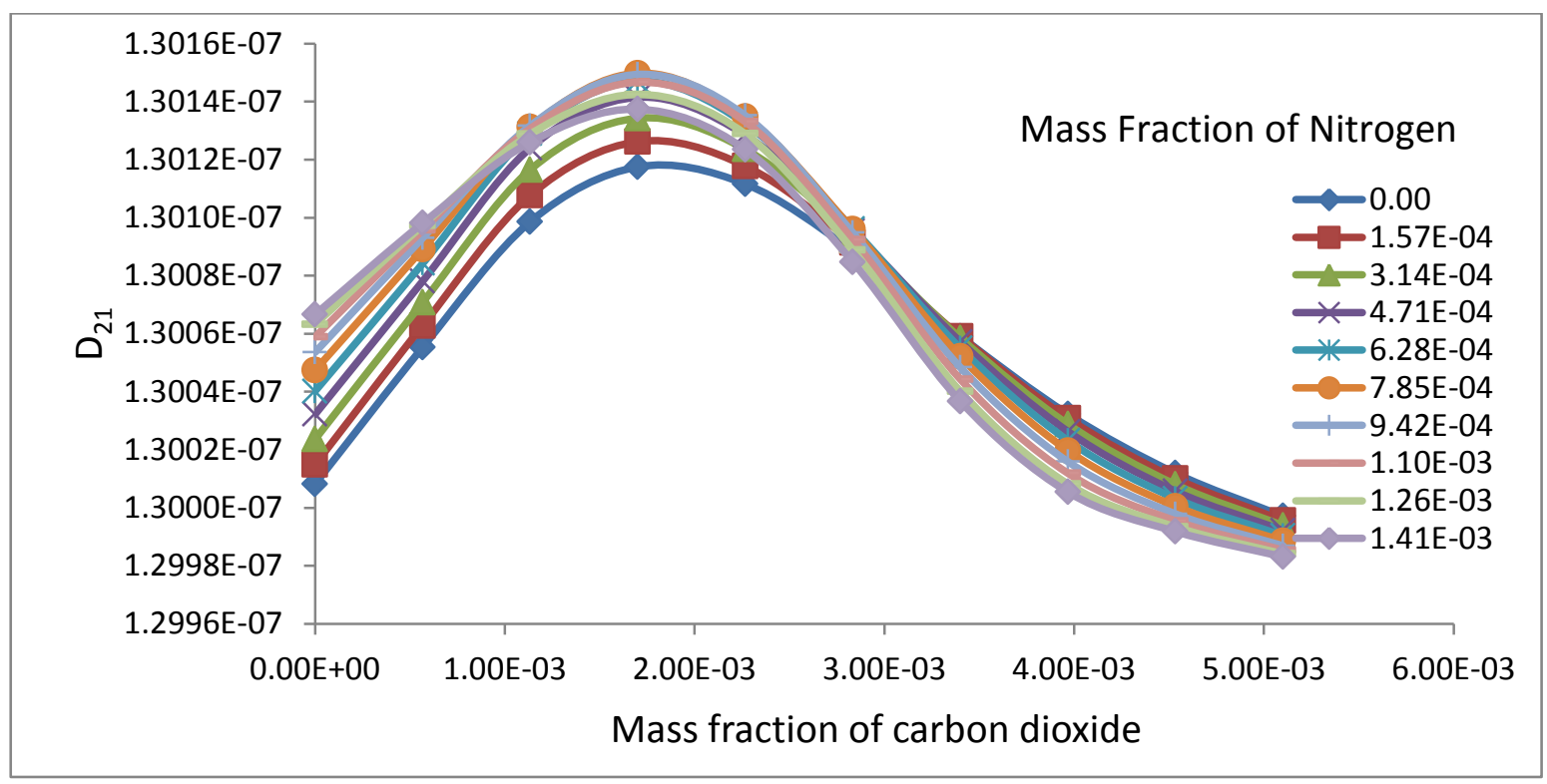

Figure 5.8. Cross-diffusion coefficient, $D_{21}$, of carbon dioxide in polystyrene

Figure 5.9 shows that the main diffusion coefficient, $D_{22}$, which increases as the concentrations of nitrogen and carbon dioxide increases.

The main diffusion coefficient $D_{22}$ remained high with low concentrations of carbon dioxide. The diffusivity was found to be on the order of $10^{-7} \mathrm{~m}^{2} \mathrm{~s}^{-1}$. The above results are in accordance with the findings of Biesenberger, 1983, who reported concentrationdependant diffusion coefficients of ethyl benzene in molten polystyrene and found that a 
very small amount of solvent can cause a very large increase in the diffusion coefficient. This behavior is particularly prominent at low concentrations of the solvent. The main diffusion coefficient, $D_{22}$, decreased gradually after the mass fraction of carbon dioxide reached $1.13 \times 10^{-3} \mathrm{~m}^{2} \mathrm{~s}^{-1}$, and the coefficient became stable at the value of $3.24 \times$ $10^{-7} \mathrm{~m}^{2} / \mathrm{s}$ when the mass fraction of carbon dioxide reached $5.10 \times 10^{-3}$.

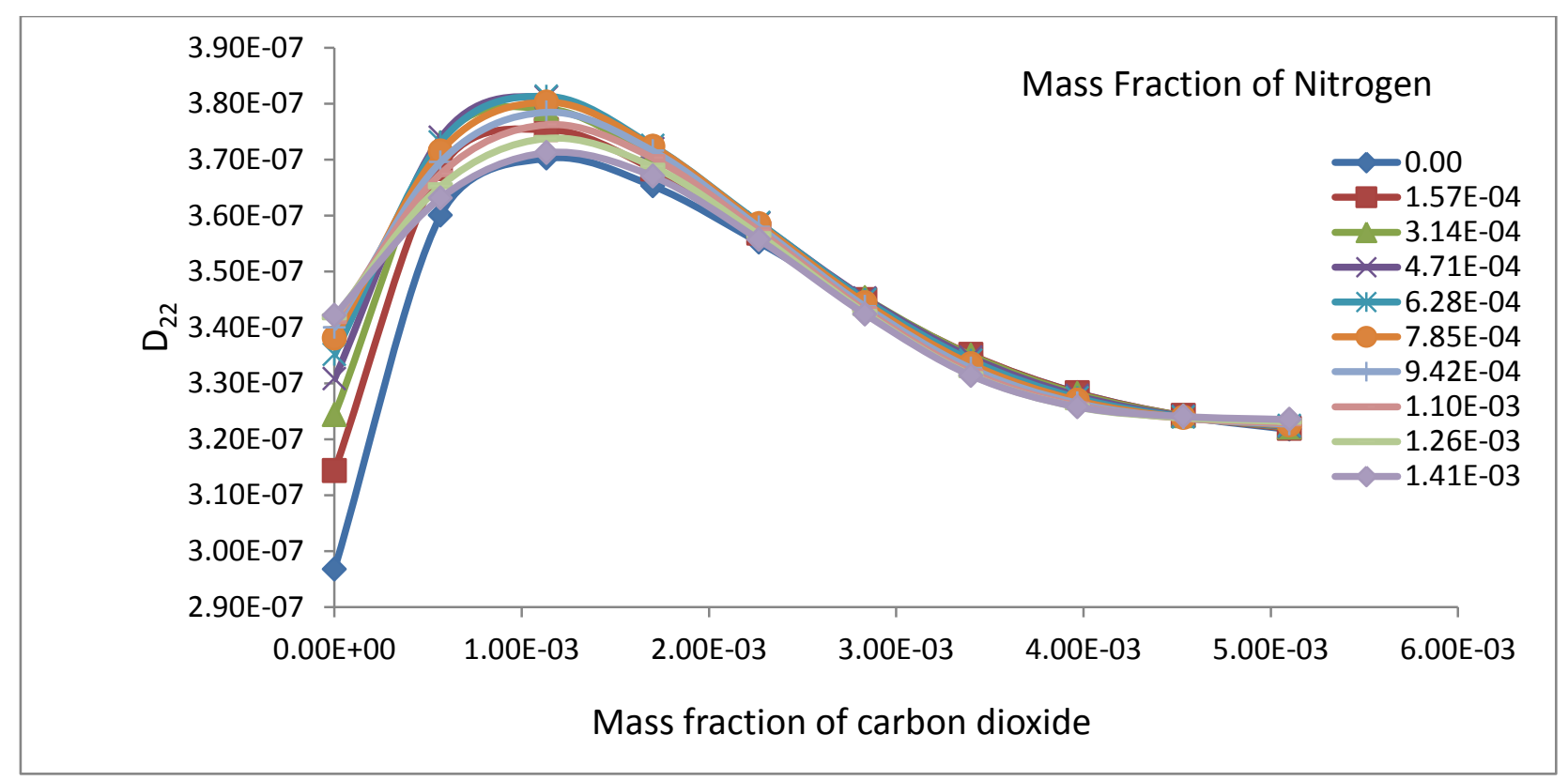

Figure 5.9. Main diffusion coefficient, $D_{22}$, of carbon dioxide in polystyrene

The ratio of cross diffusion coefficient of nitrogen $D_{12}$ to the main diffusion coefficient of nitrogen $D_{22}$ varies from $1.01 \times 10^{-2}$ to $8.06 \times 10^{-3}$, while the cross diffusion coefficient of carbon dioxide $D_{21}$ to the main diffusion coefficient of carbon dioxide $D_{11}$ varies from $1.06 \times 10^{9}$ to 5.85 . It can be concluded that, for the diffusion of nitrogen (a smaller molecule than carbon dioxide), the cross diffusion coefficient $D_{12}$ due to the concentration gradient of carbon dioxide is smaller than that for the cross diffusion coefficient of carbon 
dioxide (a larger molecule) $D_{21}$ due to the concentration gradient of nitrogen (a smaller molecule), as shown in Figures 5.10 and 5.11. Our findings are in accordance with the findings of E. L. Cussler \& Breuer, 1972, where acetone a smaller molecule, dragged the bigger molecule, sodium sulfate, and resulted in increased diffusion of sodium sulfate (bigger molecule).

But these results are in contrast to the findings of Lin et al., 2009, they found that the diffusion of di-ethanol amine (a smaller molecule, compared to methyl di-ethanol amine), the cross-diffusion effect $\left(D_{12}\right)$ due to the concentration gradient of methyl di-ethanol amine is larger than that for the diffusion of methyl di-ethanol amine (a larger molecule) $\left(D_{21}\right)$ due to the concentration gradient of di-ethanol amine (a smaller molecule).

Since multi-component diffusion is a complex process, further study is needed on the effect of molecular size, polarity, kinetic energy of molecules, dipole moment, density, viscosity, temperature, and pressure on multi-component diffusion.

In general, the diffusivity of carbon dioxide in polystyrene was found to be greater than that of nitrogen, which was in accordance with the findings of other researchers who used carbon dioxide and nitrogen separately in polypropylene and LDPE systems (Jitendra et al., 2009; Kundra et al., 2011). 


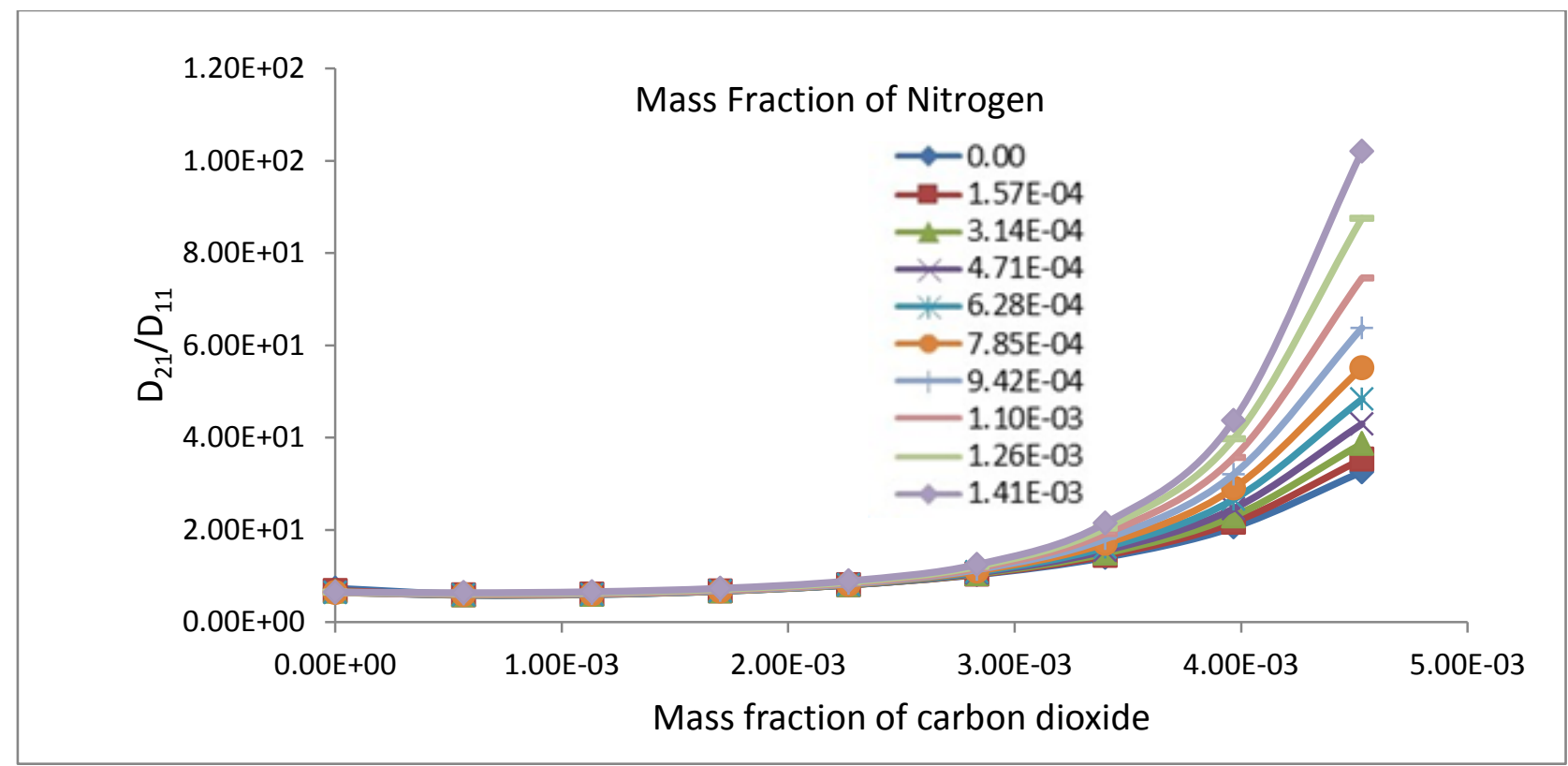

Figure 5.10. Ratio of cross diffusion coefficient $\left(D_{21} / D_{11}\right)$ of carbon dioxide due to the concentration gradient of nitrogen

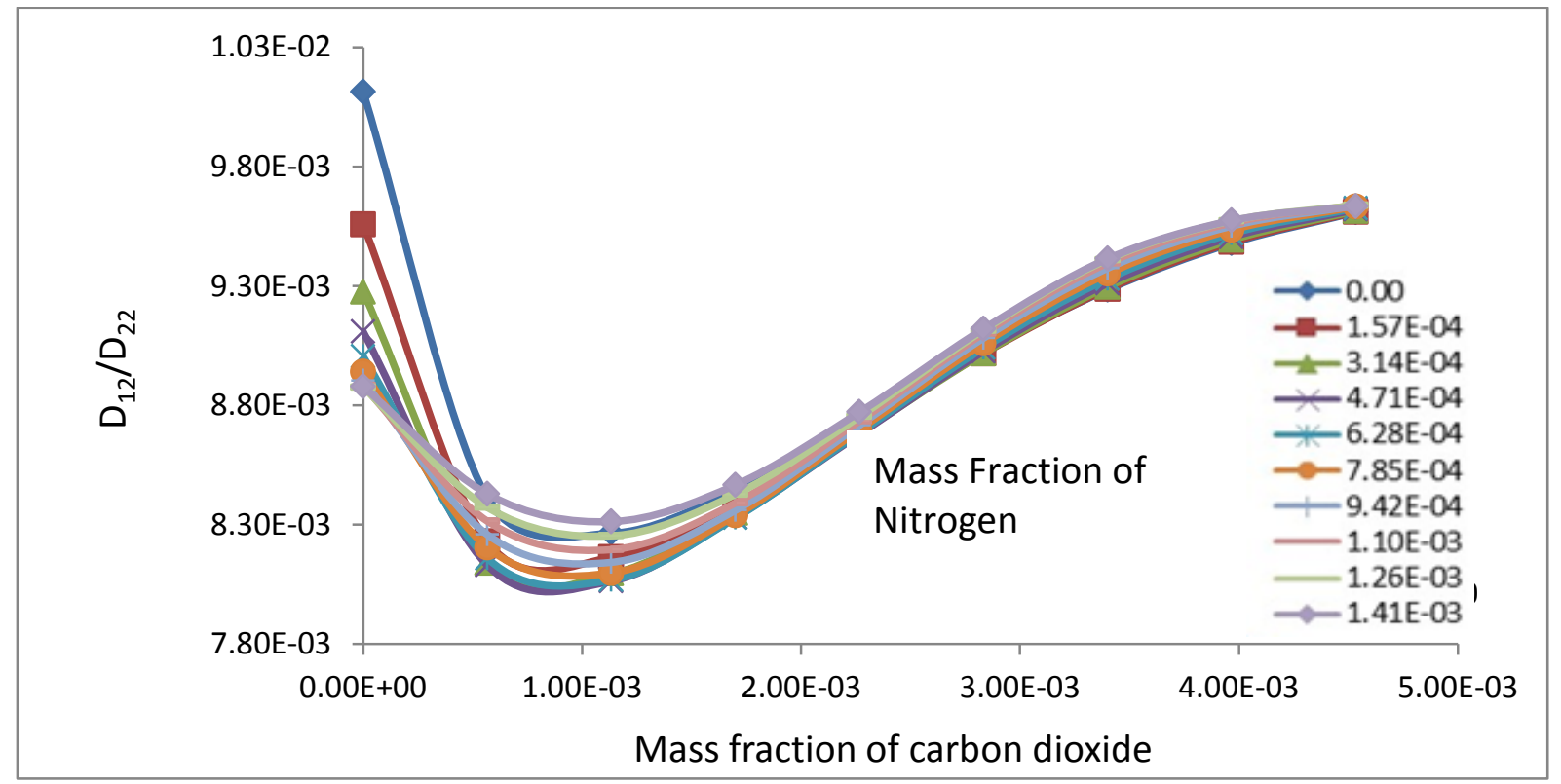

Figure 5.11. Ratio of cross diffusion coefficient $\left(D_{12} / D_{22}\right)$ of nitrogen due to the concentration gradient of carbon dioxide 


\subsection{Diffusivity Correlations}

To develop a mathematical correlation for diffusivity as a function of gas composition, we used Table curve 3D software developed by (SYSTAT Software, Inc.). The results shown in Figures 5.6-5.9 were fitted, and the best functions with the fewest parameters are given in Equations (5.1-5.4).

$$
\begin{aligned}
& D_{11}=\mathrm{a}+\mathrm{bx}+\mathrm{cy}+\mathrm{dx}^{2}+\mathrm{ey}^{2}+\mathrm{fxy}+\mathrm{gx}^{3}+\mathrm{hy}^{3}+\mathrm{ixy}^{2}+\mathrm{jx}^{2} \mathrm{y} \\
& D_{12}=\mathrm{a}+\mathrm{bx}+\mathrm{cy}+\mathrm{dx}^{2}+\mathrm{ey}^{2}+\mathrm{fxy}+\mathrm{gx}^{3}+\mathrm{hy}^{3}+\mathrm{ixy}^{2}+\mathrm{jx}^{2} \mathrm{y} \\
& D_{21}=\mathrm{a}+\mathrm{bx}+\mathrm{cx}^{2}+\mathrm{dx}^{3}+\mathrm{ex}^{4}+\mathrm{fx}^{5}+\mathrm{gy}+\mathrm{hy}^{2}+\mathrm{iy}^{3}+\mathrm{jy}^{4}+\mathrm{ky}^{5} \\
& D_{22}=\mathrm{a}+\mathrm{bx}+\frac{\mathrm{c}}{\mathrm{y}}+\mathrm{dx}^{2}+\frac{\mathrm{e}}{\mathrm{y}^{2}}+\frac{\mathrm{fx}}{\mathrm{y}}+\mathrm{gx^{3 }}+\frac{\mathrm{h}}{\mathrm{y}^{3}}+\frac{\mathrm{ix}}{\mathrm{y}^{2}}+\frac{\mathrm{jx}^{2}}{\mathrm{y}}
\end{aligned}
$$

The variables $\mathrm{x}$ and $\mathrm{y}$ are the compositions of nitrogen and carbon dioxide respectively.

Table 5.3 lists all the fitting parameters and details for Equations (5.1-5.4). The mass of absorbed gases predicted by Equations (5.1-5.4) agrees well with that based on the optimally-determined diffusivity and the experimental data.

\subsection{Sensitivity Analysis}

The final results obtained in this study were tested for sensitivity of the initial diffusivities guesses. They were varied by $\pm 3 \%$ to find the effect on the final results. The results are summarized in Table 5.4. The plots are given in Appendix D. It was found that the trend of diffusivities stays the same and the maximum values do not change by more than $10 \%$. 
Table 5.3. Parameters for diffusivity correlation at $23^{\circ} \mathrm{C}$ and $224 \mathrm{kPa}$

\begin{tabular}{|c|c|c|c|c|}
\hline Parameter & $D_{11}$ & $D_{12}$ & $D_{21}$ & $D_{22}$ \\
\hline \multirow[t]{2}{*}{$\mathrm{a}$} & 1.845 & 2.9921 & $2 \times 10^{-7}$ & $4.4846 \times 10^{-7}$ \\
\hline & $\times 10^{-8}$ & $\times 10^{-9}$ & & \\
\hline \multirow[t]{2}{*}{$\mathrm{b}$} & 5.4227 & 4.4420 & $-2.9210 \times 10^{-10}$ & $1.9901 \times 10^{-3}$ \\
\hline & $\times 10^{-6}$ & $\times 10^{-8}$ & & \\
\hline \multirow[t]{2}{*}{ C } & 6.3573 & 7.8767 & $2.9314 \times 10^{-6}$ & 0 \\
\hline & $\times 10^{-6}$ & $\times 10^{-8}$ & & \\
\hline \multirow[t]{2}{*}{$d$} & 4.2836 & 1.4516 & -0.0079 & -1.6671 \\
\hline & $\times 10^{-3}$ & $\times 10^{-5}$ & & \\
\hline \multirow[t]{2}{*}{$\mathrm{e}$} & 4.3025 & 1.7408 & 6.6268 & 0 \\
\hline & $\times 10^{-3}$ & $\times 10^{-5}$ & & \\
\hline \multirow[t]{2}{*}{$f$} & 2.3475 & 8.1539 & -1823.0442 & 0 \\
\hline & $\times 10^{-3}$ & $\times 10^{-6}$ & & \\
\hline \multirow[t]{2}{*}{ g } & 7.0425 & 2.3026 & $-4.3135 \times 10^{-10}$ & 408.0278 \\
\hline & $\times 10^{-1}$ & $\times 10^{-3}$ & & \\
\hline \multirow[t]{2}{*}{$\mathrm{h}$} & 4.7957 & 1.3150 & $8.1928 \times 10^{-7}$ & 0 \\
\hline & $\times 10^{-1}$ & $\times 10^{-3}$ & & \\
\hline \multirow[t]{2}{*}{ i } & 1.8472 & 4.3068 & -0.0003 & 0 \\
\hline & $\times 10^{-1}$ & $\times 10^{-4}$ & & \\
\hline \multirow[t]{2}{*}{ j } & 6.5648 & 6.9709 & -0.0214 & 0 \\
\hline & $\times 10^{-1}$ & $\times 10^{-4}$ & & \\
\hline $\mathrm{k}$ & 0 & 0 & 8.7533 & 0 \\
\hline \multirow{3}{*}{$\begin{array}{l}r^{2} \text { Coefficient } \\
\text { of } \\
\text { Determination }\end{array}$} & 0.9998 & 0.9985 & 0.9907 & 1 \\
\hline & & & & \\
\hline & & & & \\
\hline \multirow{2}{*}{$\begin{array}{l}\text { Fit standard } \\
\text { error }\end{array}$} & 2.9329 & 4.4529 & $4.0326 \times 10^{-13}$ & 0 \\
\hline & $\times 10^{-10}$ & $\times 10^{-12}$ & & \\
\hline F-statistic & 7502.8440 & 678.5260 & 951.8373 & $1 \times 10^{300}$ \\
\hline
\end{tabular}


Table 5.4 . Sensitivity analysis $\pm 3 \%$ change in the estimate

\begin{tabular}{|c|c|c|c|c|c|c|}
\hline & $\begin{array}{l}\text { Initial } \\
\text { estimate }\end{array}$ & $\begin{array}{l}\text { Output with initial } \\
\text { estimate }\end{array}$ & $\begin{array}{l}\text { Output with }+3 \% \\
\text { increase in the estimate }\end{array}$ & $\begin{array}{l}\% \\
\text { change }\end{array}$ & $\begin{array}{l}\text { Output with }-3 \% \\
\text { decrease in the estimate }\end{array}$ & $\begin{array}{l}\% \\
\text { Change }\end{array}$ \\
\hline$D_{11}$ & $3.0 \times 10^{-9}$ & $\begin{array}{l}2.22 \times 10^{-08} \text { to } \\
1.22 \times 10^{-16}\end{array}$ & $\begin{array}{l}2.32 \times 10^{-8} \text { to } \\
1.29 \times 10^{-16}\end{array}$ & $\begin{array}{l}4.50 \text { to } \\
5.73\end{array}$ & $\begin{array}{l}2.00 \times 10^{-08} \\
10^{-16}\end{array}$ to $1.25 \times$ & $\begin{array}{l}-9.99 \text { to } \\
2.45\end{array}$ \\
\hline$D_{12}$ & $1.0 \times 10^{-8}$ & $\begin{array}{l}3.13 \times 10^{-09} \text { to } \\
3.0 \times 10^{-9}\end{array}$ & $\begin{array}{l}3.22 \times 10^{-9} \text { to } \\
3.09 \times 10^{-9}\end{array}$ & $\begin{array}{l}2.87 \text { to } \\
3\end{array}$ & $\begin{array}{l}2.83 \times 10^{-09} \text { to } 2.71 \times \\
10^{-9}\end{array}$ & $\begin{array}{l}-9.58 \text { to }- \\
9.66\end{array}$ \\
\hline$D_{21}$ & $1.30 \times 10^{-7}$ & $\begin{array}{l}1.30 \times 10^{-07} \text { to } \\
1.29 \times 10^{-7}\end{array}$ & $\begin{array}{l}1.34 \times 10^{-7} \text { to } \\
1.33 \times 10^{-7}\end{array}$ & 3 to 3 & $\begin{array}{l}1.175 \times 10^{-07} \\
\text { to } 1.17 \times 10^{-7}\end{array}$ & $\begin{array}{l}-9.71 \text { to } \\
9.699\end{array}$ \\
\hline$D_{22}$ & $3.5 \times 10^{-7}$ & $\begin{array}{l}3.81 \times 10^{-07} \text { to } \\
2.97 \times 10^{-7}\end{array}$ & $\begin{array}{l}4.10 \times 10^{-7} \text { to } 3.14 \times \\
10^{-7}\end{array}$ & $\begin{array}{l}7.611 \text { to } \\
5.72\end{array}$ & $\begin{array}{l}3.53 \times 10^{-07} \text { to } \\
2.76 \times 10^{-7}\end{array}$ & $\begin{array}{l}-7.34 \text { to } \\
7.07\end{array}$ \\
\hline
\end{tabular}




\section{CONCLUSIONS and RECOMMENDATIONS}

\subsection{CONCLUSIONS}

In this study, we derived a detailed, dynamic, mass-transfer model of the experimental diffusion process for the polymer phase to provide the temporal masses of gases in the polymer during the diffusion experiments. Due to the presence of highly non-linear partial differential equations, the analytical solution to this problem is not possible. Therefore, the optimal control strategy was used to solve this problem numerically.

For this purpose an optimal control framework was developed to determine multicomponent concentration dependent diffusivities. Necessary conditions were determined at which the error between the calculated and experimental gas masses was minimized. The satisfaction of these conditions determines the unknown diffusivities. Then, these conditions were used to develop an optimal control frame work.

The above optimal control framework was applied to the ternary system of nitrogen (1) and carbon dioxide (2) in polystyrene (3) at room temperature $\left(23^{\circ} \mathrm{C}\right)$ and a pressure of 224 $\mathrm{kPa}$. Solubility and diffusion experiments data were used along with the detailed mass 
transfer model and optimal control framework to calculate the multi-component diffusivities of nitrogen and carbon dioxide in polystyrene as a function of their compositions. The diffusivity of carbon dioxide was found to be greater than that of nitrogen. The value of the main diffusion coefficient $D_{11}$ was found to increase as the concentration of carbon dioxide increased. The highest value of $D_{11}$ obtained was $2.22 \times 10^{-8} \mathrm{~m}^{2} / \mathrm{s}$ for nitrogen mass fraction of $3.14 \times 10^{-4}$ and for a carbon dioxide mas fraction of $5.67 \times 10^{-4}$. The $D_{11}$ decreased after the mass fraction carbon dioxide mass fraction of $5.67 \times 10^{-4}$. The diffusivity varied in the range of $10^{-8}$ to $10^{-9} \mathrm{~m}^{1} \mathrm{~s}^{-1}$. The cross-diffusion coefficient $D_{12}$, increased as the concentrations of nitrogen and carbon dioxide increased. The diffusivity reached its maximum value when the concentrations of nitrogen and carbon dioxide were at their maximum values. The diffusivity was of the order of $10^{-9} \mathrm{~m}^{2} \mathrm{~s}^{-1}$

A trend similar to that of $D_{11}$ was observed with cross-diffusion coefficient $D_{21}$. The diffusivity of carbon dioxide increased for the mass fractions of carbon dioxide ranging from 0 to $1.70 \times 10^{-3}$. The diffusivity was found to be of the order of $10^{-7} \mathrm{~m}^{2} \mathrm{~s}^{-1}$. After the carbon dioxide mass fraction reached $1.70 \times 10^{-3}$, the diffusivity decreased as the mass of carbon dioxide increased, reaching a minimum at the carbon dioxide mass fraction of $5.10 \times 10^{-3}$.

The diffusion coefficient, $D_{22}$, was found to increase with the concentrations of nitrogen and carbon dioxide. Diffusivity $D_{22}$ remained high with low concentrations of carbon dioxide. The diffusivity was found to be of the order of $10^{-7} \mathrm{~m}^{2} \mathrm{~s}^{-1}$. 


\subsection{RECOMMENDATIONS}

This work was a first step towards producing the important, fundamental diffusivity data that are required for chemical industries. Optimal control framework developed in this study can be used to generate concentration dependent multicomponent diffusivity data for two gases and a liquid. Future experiments can be done with different gases and polymers at different temperatures and pressures. 


\section{References}

Amooghina, A. E., Shehnib, P. M., Ghadimic, A., Sadrzadehc, M., \& Mohammadib, T. (2013). Mathematical modeling of mass transfer in multicomponent gas mixture across the synthesized composite polymeric membrane. Journal of Industrial and Engineering Chemistry, 19(3), 870-885.

Arnold, K. R., \& Toor, H. L. (1967). Unsteady diffusion in ternary gas mixtures. AlCHE Journal, 13(5), 909-914.

Biesenberger, J. A. (1983). Devolatilization of polymers fundamentals of equipment applications. Hanser.

Bouchet, R., \& Mevrel, R. (27). Calculating the composition-dependent diffusivity matrix along a diffusion path in ternary systems : Application to $\beta$-(ni, pt)al. 2003, 3, 295-303.

Cussler, E. L. (Ed.). (2009). Diffusion, mass transfer in fluid systems (3rd ed.). United Kingdom: Cambridge.

Cussler, E. L., \& Lightfoot, E. N. (1965). Multicomponent diffusion involving high polymers. I. diffusion of monodisperse polystyrene in mixed solvents. The Journal of Physical Chemistry, 69(4), 1135.

Cussler, E. L., \& Peter, J. D. (1966). An experimental comparison of the gouy and the diaphragm cell methods for studying isothermal ternary diffusion. The Journal of Chemical Physics, 70(6), 1880.

Cussler, E. L., \& Breuer, M. M. (1972). Accelerating diffusion with mixed solvents. AICHE Journal, 18(4), 812-816.

Denn, M. M. (1969). Optimization by variational methods McGraw-Hill, New York.

Duda, J. L. (1985). Molecular diffusion in polymeric systems. Pure and Applied Chemistry, 57(11), 1681.

Duda, J. L., Ni, Y. C., \& Vrentas, J. S. (1978). Diffusion of ethylbenzene in molten polystyrene. Journal of Applied Polymer Science, 22(3), 689-699.

Felder, R. M., \& Huvard, G. S. (1980). Methods in experimental physics. (pp. 315)

Fick, A. (1995). On liquid diffusion . Journal of Membrane Science, Elsevier(100), 33-38. 
Ghoreyshi, A. A., Farhadpour, F. A., \& Soltanieh, M. (2004). A general model for ulticomponent transport in nonporous membranes based on Maxwell-Stefan formulation. hemicalEngineering Communications, 191(4), 460-499.

Graham, T. (1833). On the law of diffusion of gases. Philosophical Magazine, 2(9)

Graham, T. (1850). On the diffusion of liquids. Philosophical Transactions of the Royal Society A, 140, 1-46.

Jitendra, T., Upreti, R. Simant., \& Lohi, A. (2009). Experimental determination of oncentration-dependent carbon dioxide diffusivity in LDPE. Journal of Applied Polymer Science, 111(1), 380.

Kirk, D. E. (1970). Optimal control theory, an introduction. New York, USA: Dover Publications.

Kjetil, B., Haugen, \& Firoozabadi, A. (2006). On measurement of molecular and thermal diffusion coefficients in multicomponent mixtures. The Journal of Physical Chemistry B, 110(35), 17678-17682.

Krishnamurthy, R., \& Taylor, R. (1982). Calculation of multicomponent mass tranfer at high tranfer rates. The Chemical Engineering Journal, 25(1), 47-54.

Kundra, P., Upreti, R., Simant., Ali, L., \& Jiangning, W. (2011). Experimental determination of composition-dependent diffusivity of carbon dioxide in polypropylene. Journal of Chemical \& Engineering Data, 56(1), 21-26.

Kwag, C., Manke, C. W., \& and Gulari, E. (1999). Rheology of molten polystyrene with dissolved supercritical and near-critical gases. Journal of Polymer Science Part B: Polymer Physics, Volume 37(19), 2771-2781.

Li, D. C., Liu, T., Zhao, L., \& Yuan, W. (2009). Solubility and diffusivity of carbon dioxide in solid-state isotactic polypropylene by the Pressure-Decay method. Industrial \& Engineering Chemistry Research, 48(15), 7117-7124.

Lin, P., Ko, C., \& Ko, M. (2009). Ternary diffusion coefficients of diethanolamine and Nethyldiethanolamine in aqueous solutions containing diethanolamine and $\mathrm{N}$ ethyldiethanolamine. Fluid Phase Equilibria, 276(1), 69-74.

Liu, X., Vlugt, T. J. H., \& Bardow, A. (2011). Predictive darken equation for maxwell-stefan diffusivities in multicomponent mixtures. Industrial \& Engineering Chemistry Research, 50(17), 10350-10358.

Lundberg, J. L. (1964a). Journal of Polymer Science Part A: General Papers, 2(9), 3925. 
Lundberg, J. L. (1964b). Diffusivities and solubilities of methane in linear polyethylene melts. Journal of Polymer Science Part A: General Papers, 2(9), 3925.

Lundberg, J. L., Wilk, M. B., \& Huyett, M. J. (1963). Sorption studies using automation and computation. Industrial \& Engineering Chemistry Fundamentals, 2(1), 37.

Lundberg, J. L., Wilk, M. B., \& Huyett, M. J. (1962). Estimation of diffusivities and solubilities from sorption studies. Journal of Polymer Science, 57(165), 275.

Lundberg, J. L., Wilk, M. B., \& Huyett, M. J. (1960). Solubilities and diffusivities of nitrogen in polyethylene. Journal of Applied Physics, 31(6), 1131.

Masom, E. A., \& and Kronstadt, B. (1967). Graham's laws of diffusion and effusion E. A. mason and barbara kronstadt journal of chemical Education 196744 (12), 740 Journal of Chemical Education, 44(12), 740.

Moradi Shehni, P., Amooghin, A. E., Ghadimi, A., Sadrzadeh, M., \& Mohammadi, T. (2011). Modeling of unsteady-state permeation of gas mixture through a self-synthesized PDMS membranesElsevier.

Nikitin, L. N., Gallyamov, M. O., Vinokur, R. A., Nikolaec, A. Y., Said-Galiyev, E. E., Khokhlov, A. R., et al. (2003). Swelling and impregnation of polystyrene using supercritical carbon dioxide. The Journal of Supercritical Fluids, 26(3), 263-273.

Philibert, J. (2005). One and a half century of diffusion: Fick, einstein, before and beyond. The Open-Access Journal for the Basic Principles of Diffusion Theory, Experiment and Application, 2, 1.1-1.10.

Ramirez, F. W. (1987). Developments in petroleum science 21, application of optimal control theory to enhanced oil recovery. Elsevier, New York.

Ray, W. H. (1981). Advanced process control. New York: McGraw-Hill.

Rehfeldt, S., \& Stichlmair, J. (2007). Prediction of multicomponent diffusion coefficients in liquids. AIChE Annual Meeting, Conference Proceedings, Spring National Meeting; Houston, TX; United States; Code 74096,

Sato, Y., Takikawa, T., Takishima, S., \& Masuoka, H. (2001). Solubilities and diffusion coefficients of carbon dioxide in poly(vinyl acetate) and polystyrene. The Journal of Supercritical Fluids, 19(2), 187-198.

Sato, Y., Yurugi, M., Fujiwara, K., Takishima, S., \& Masuoka, H. (1996). Solubilities of carbon dioxide and nitrogen in polystyrene under high temperature and pressure. Fluid Phase Equilibria, 125(1-2), 129-138. 
Surat, A., Eita, F., Yusuke, H., Dai, N., \& Masahiro, O. (2004). Measurement and prediction of diffusion coefficients of supercritical $\mathrm{CO}_{2}$ in molten polymers. Polymer Engineering and Science, 44(10), 1915.

Taylor, R., \& Krishna, R. (Eds.). (1993). Amsterdam: Wiley Interscience.

Telis, V. R. N., Murarif. R.C.B.D.L., \& Yamashita, F. (2004). Diffusion coefficients during osmotic dehydration of tomatoes in ternary solutions. Journal of Food Engineering, 61(2), 253-259.

Upreti, R., Simant. (2013). Optimal control for chemical engineers (1st ed.). New York: CRC Press Taylor and Francis Group.

Upreti, R., Simant., \& Mehrotra, A., K. (2000). Experimental measurement of gas diffusivity in bitumen: results for carbon dioxide. Industrial \& Engineering Chemistry Research, 39(4), 1080.

Vrentas, J. S., Duda, J. L., \& Lau, M. K. (1982). Solvent diffusion in molten polyethylene. Journal of Applied Polymer Science, 27(10), 3987.

Williams, J. W., \& Cady, L. C. (1934). Molecular diffusion in solution. Chemical Reviews, 14(2), 171. 
Appendix A 


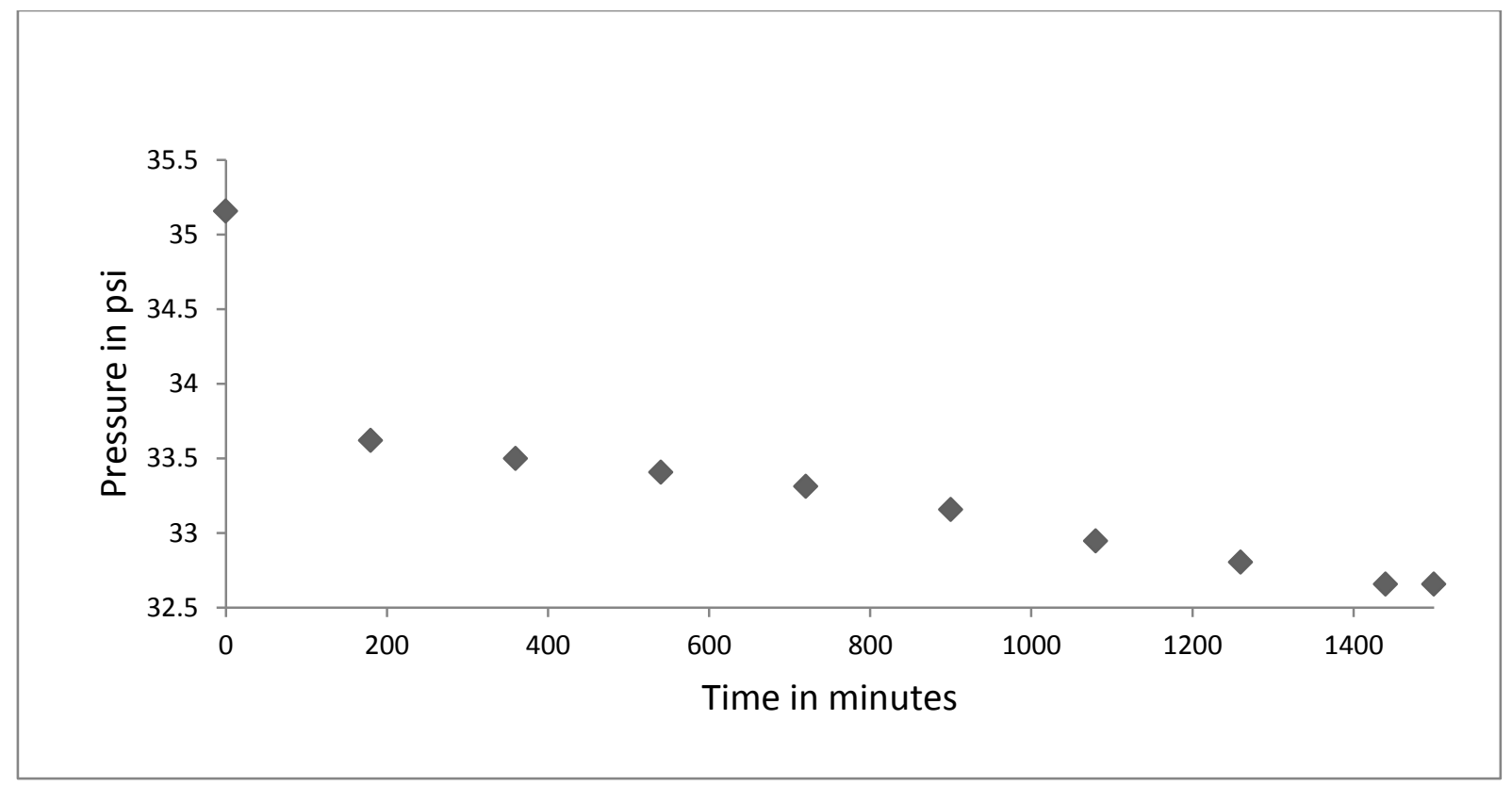

A 1 Pressure versus time plot of solubility experiment

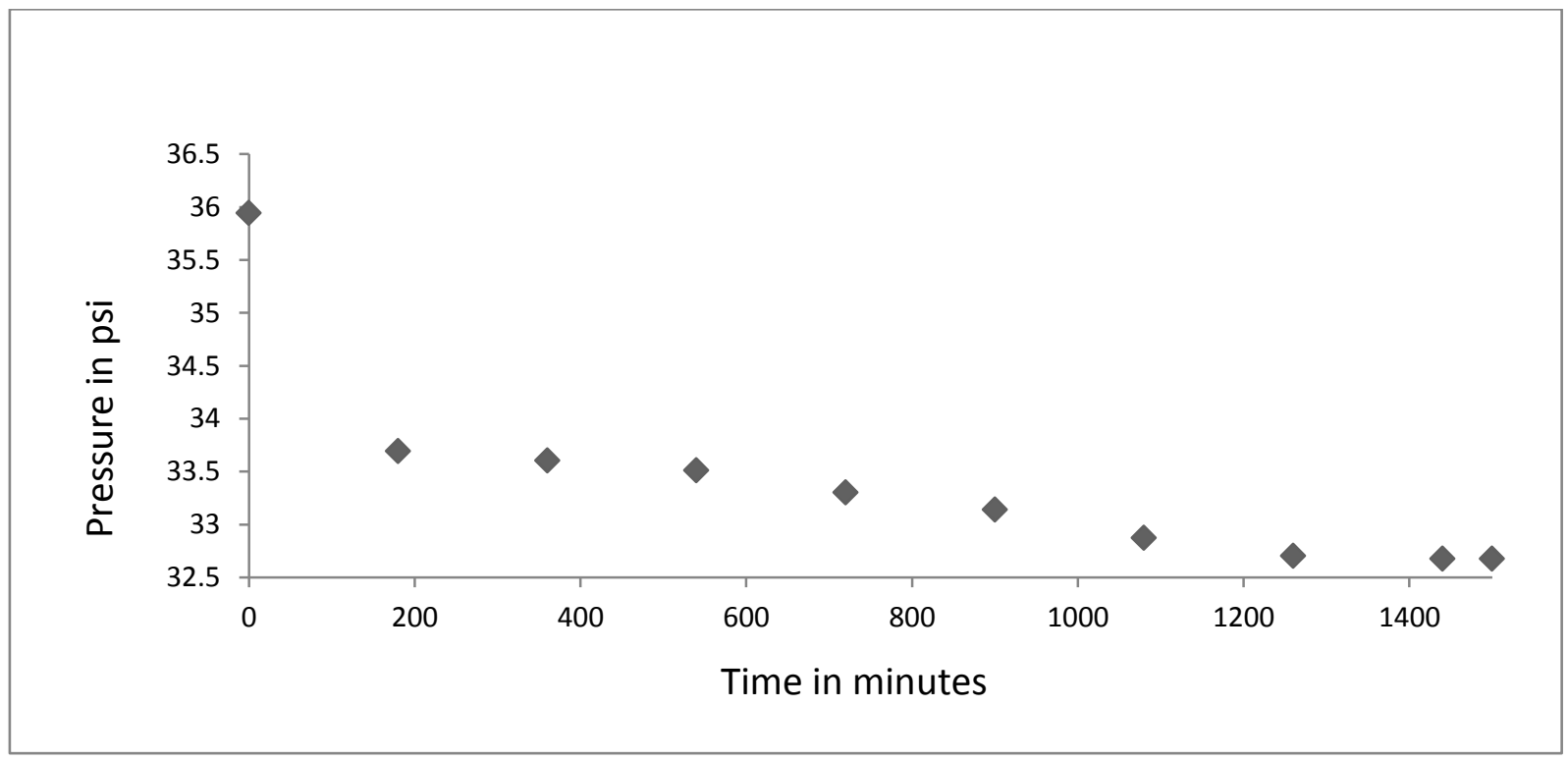

A 2 Pressure versus time plot of solubility experiment 


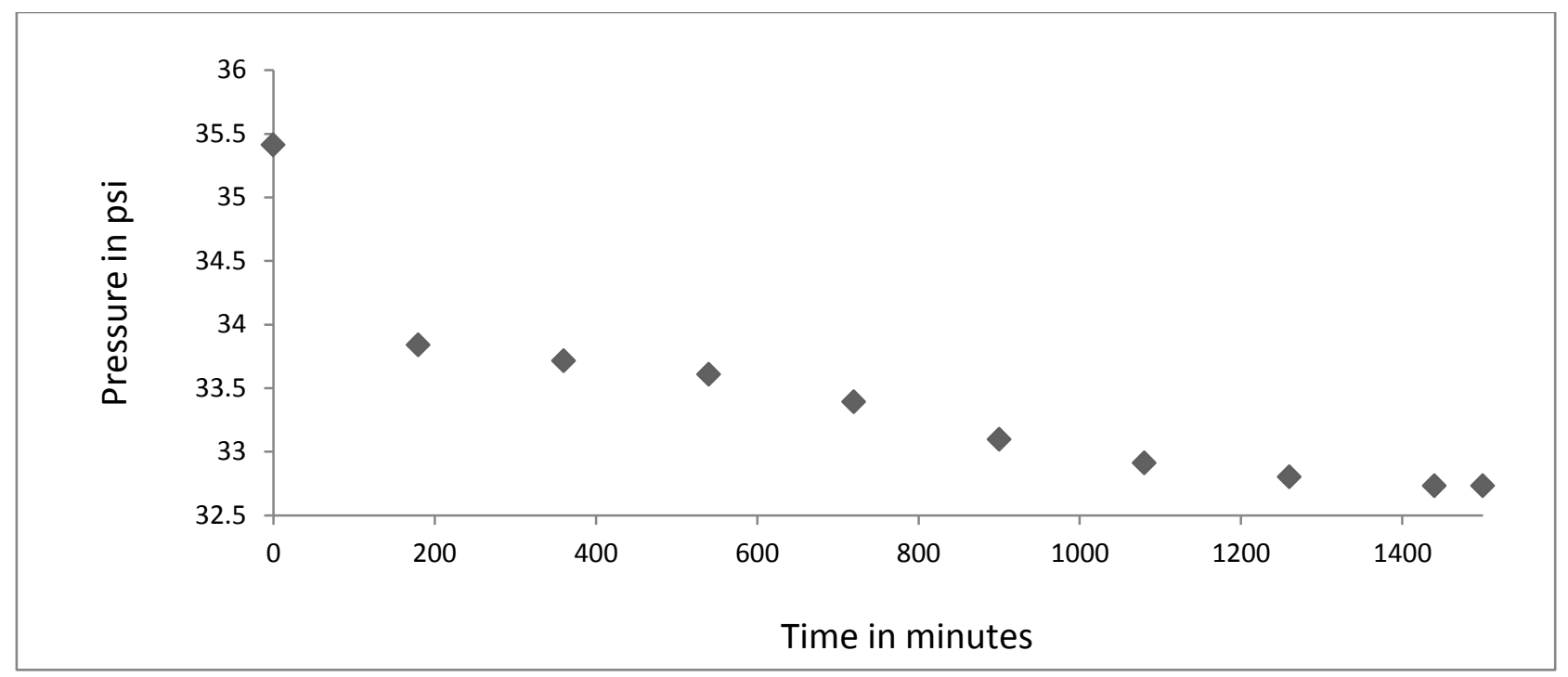

A 3 Pressure versus time plot of solubility experiment

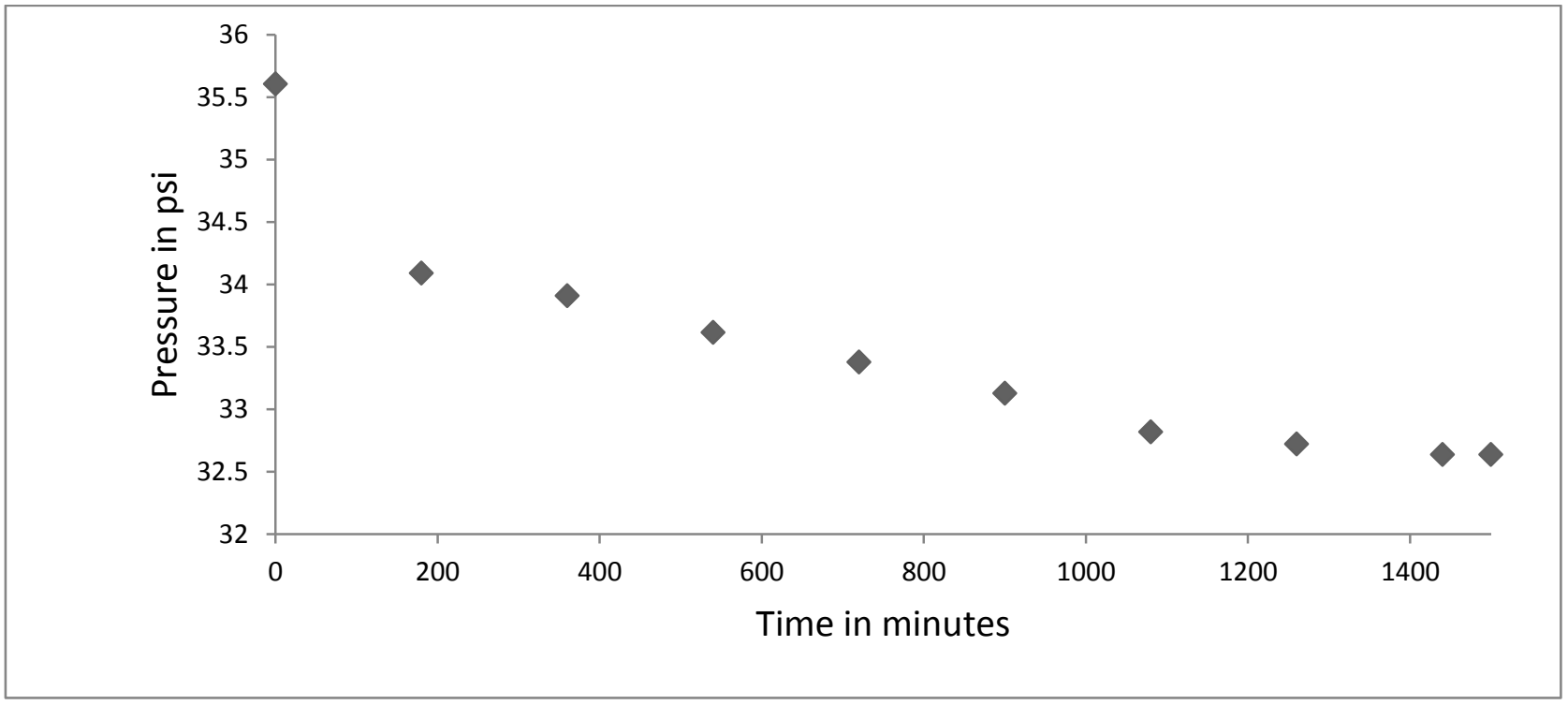

A 4 Pressure versus time plot of solubility experiment 
Appendix B 


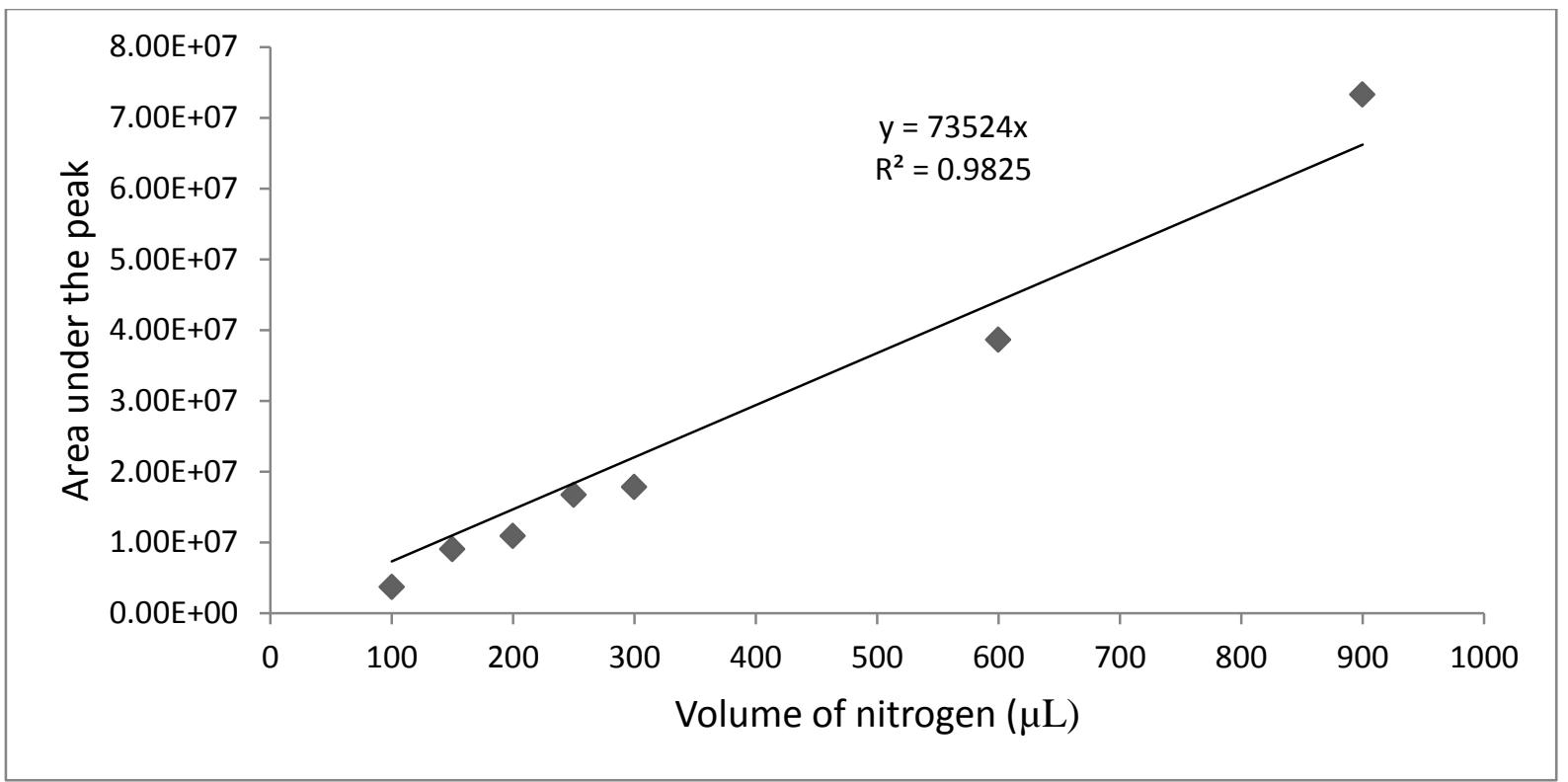

B 1 Straight line fit for nitrogen

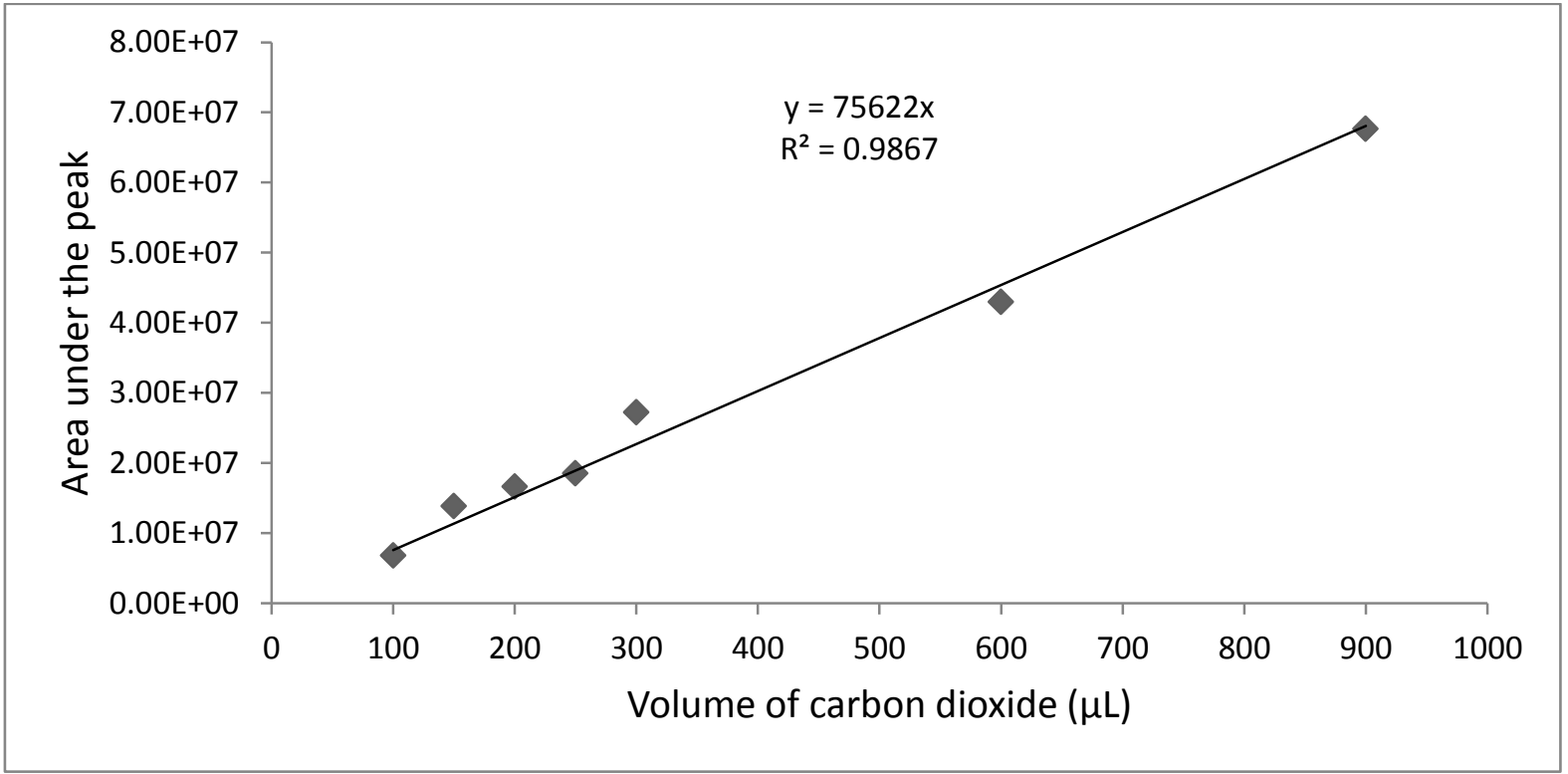

B 2 Straight line fit for carbon dioxide 
Table B 1 Calculation of masses of two gases in the sample during diffusion experiments

\begin{tabular}{|c|c|c|c|c|}
\hline $\mathbf{N}_{2}$ & $\begin{array}{l}\text { Area Under } \\
\text { the Curve }\end{array}$ & $\mathbf{L}$ & g & $\mathrm{g} / \mathrm{L}$ \\
\hline Initial & 6083656.314 & 0.00008274 & 0.000207978 & 1.039890968 \\
\hline $2 \mathrm{~min}$ & 5540370.264 & 0.00007535 & 0.000189405 & 0.947026048 \\
\hline $4 \mathrm{~min}$ & 5119394.174 & 0.00006962 & 0.000175014 & 0.875067803 \\
\hline $6 \mathrm{~min}$ & 4264247.514 & 0.00005799 & 0.000145779 & 0.728895954 \\
\hline $8 \mathrm{~min}$ & 4081304.214 & 0.00005550 & 0.000139525 & 0.697625107 \\
\hline $10 \mathrm{~min}$ & 4001164.664 & 0.00005441 & 0.000136785 & 0.683926701 \\
\hline \multicolumn{5}{|l|}{$\mathrm{CO}_{2}$} \\
\hline Initial & 7800756.09 & 0.00010315 & 0.000414931 & 2.074654067 \\
\hline $2 \mathrm{~min}$ & 6804815.13 & 0.00008998 & 0.000361956 & 1.80977808 \\
\hline $4 \mathrm{~min}$ & 6382529.03 & 0.00008440 & 0.000339494 & 1.697468765 \\
\hline $6 \mathrm{~min}$ & 4502618.32 & 0.00005954 & 0.000239499 & 1.197496153 \\
\hline $8 \mathrm{~min}$ & 3628367.43 & 0.00004798 & 0.000192997 & 0.964984311 \\
\hline $10 \mathrm{~min}$ & 3326615.03 & 0.00004399 & 0.000176946 & 0.884731598 \\
\hline
\end{tabular}


Table B 2 Calculation of masses of two gases in the sample during solubility experiments

\begin{tabular}{|c|c|c|c|c|c|c|}
\hline $\mathbf{N}_{2}$ & $\begin{array}{l}\text { Area Under } \\
\text { the Curve } \\
\text { Initial }\end{array}$ & $\begin{array}{l}\text { Area Under } \\
\text { the Curve } \\
\text { Final }\end{array}$ & $\begin{array}{l}\text { Volume in } \\
\text { Liters Initial }\end{array}$ & $\begin{array}{l}\text { Volume in } \\
\text { Liters } \\
\text { Final }\end{array}$ & $\begin{array}{l}\text { Mass in g } \\
\text { Initial }\end{array}$ & $\begin{array}{l}\text { Mass in } \mathbf{g} \\
\text { Final }\end{array}$ \\
\hline & 30338453.29 & 24670022.39 & 0.000412633 & 0.000335537 & 0.001037162 & 0.000843379 \\
\hline & 23420261.44 & 22470110.78 & 0.000318539 & 0.000305616 & 0.000800654 & 0.000768172 \\
\hline & 28023893.17 & 25468419.5 & 0.000381153 & 0.000346396 & 0.000958035 & 0.000870673 \\
\hline & 30705019.36 & 25629510.59 & 0.000417619 & 0.000348587 & 0.001049693 & 0.00087618 \\
\hline & 25323649.99 & 28981175.65 & 0.000344427 & 0.000394173 & 0.000865724 & 0.000990762 \\
\hline \multicolumn{7}{|l|}{$\mathrm{CO}_{2}$} \\
\hline & 15254318.6 & 8941923.39 & 0.000201718 & 0.000118245 & 0.000811394 & 0.000475631 \\
\hline & 15773312.38 & 8230471.614 & 0.000208581 & 0.000108837 & 0.000839 & 0.000437788 \\
\hline & 13955661.99 & 8585441.282 & 0.000184545 & 0.000113531 & 0.000742317 & 0.000456669 \\
\hline & 11838321.61 & 66258408.34 & 0.000156546 & 0.000876179 & 0.000629693 & 0.003524358 \\
\hline & 10305841.78 & 5136473.106 & 0.000136281 & 0.000067923 & 0.000548179 & 0.000273215 \\
\hline
\end{tabular}




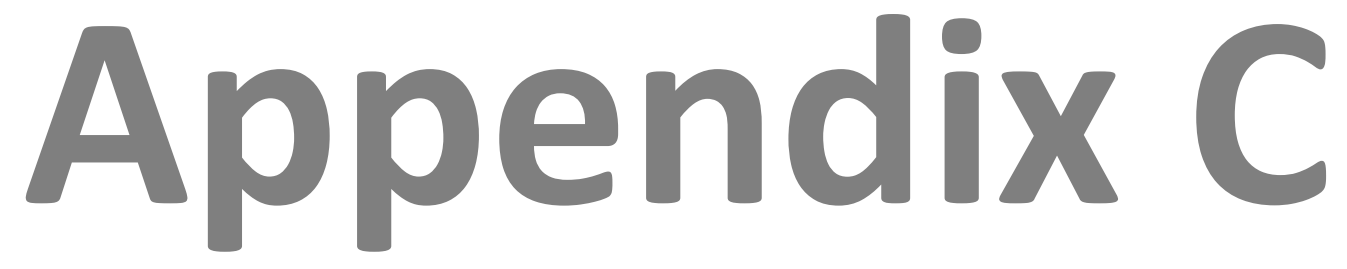




\section{Set Derivs. Cpp}

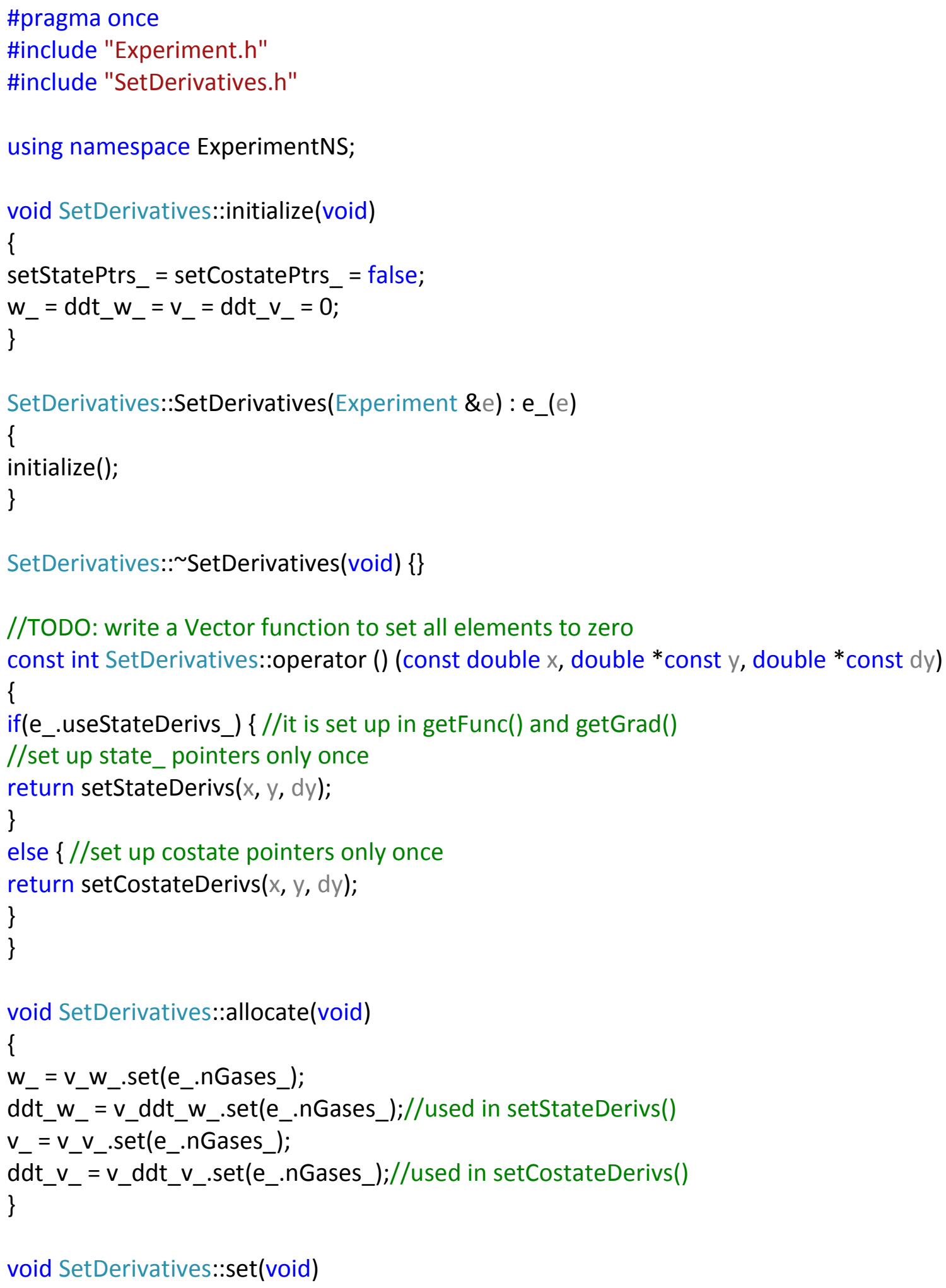




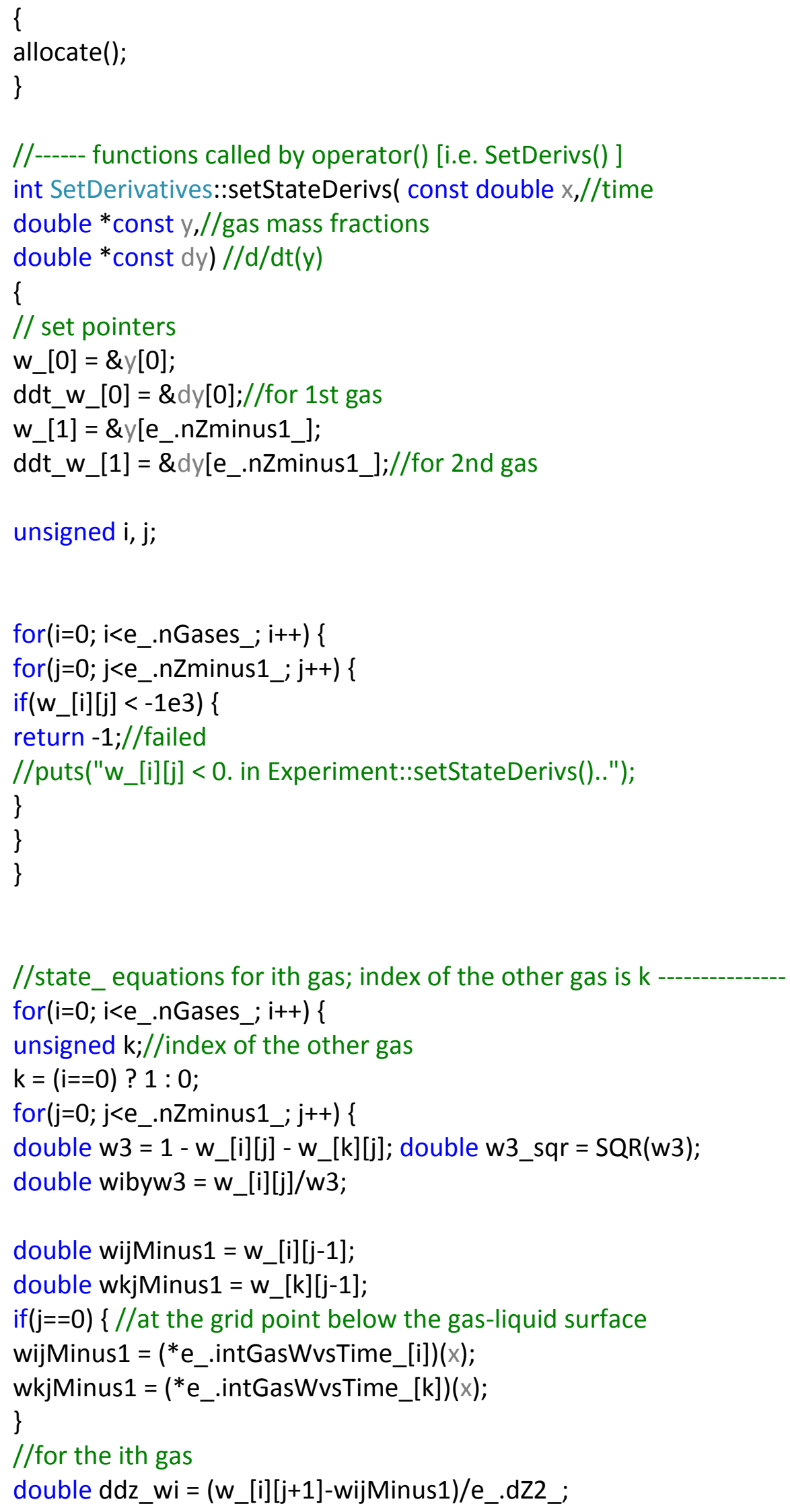


double ddz_wi_sqr=SQR(ddz_wi);

$/ /$ for the other gas of index $k$

double ddz_wk $=\left(w \_[k][j+1]-w k j M i n u s 1\right) / e_{\_} . d Z 2 \_;$

double ddz_wk_sqr $=S Q R\left(d d z \_w k\right)$;

double d2dz2_wi, d2dz2_wk;

if $\left(j<e_{-} . n Z m i n u s 2 \_\right)\{/ /$for intermediate grid points

$d 2 d z 2 \_w i=\left(w \_[i][j+1]-2 * w \_[i][j]+w i j M i n u s 1\right) / e_{-} . d Z s q r \_;$

$d 2 d z 2 \_w k=\left(w \_[k][j+1]-2 * w_{-}[k][j]+w k j M i n u s 1\right) / e_{-} . d Z s q r \_;$

\}

else $\{$ //at the bottom

$\mathrm{d} 2 \mathrm{dz2} \_w i=2^{*}\left(w_{-}[\mathrm{i}][\mathrm{j}-1]-w_{-}[\mathrm{i}][\mathrm{j}]\right) / \mathrm{e}_{-} . \mathrm{dZsqr}$;

$\mathrm{d} 2 \mathrm{dz2} \_w k=2^{*}\left(w_{-}[\mathrm{k}][j-1]-w_{-}[k][j]\right) / e_{-} . d Z s q r \_;$

\}

double Dii $=\left({ }^{*} e_{-}\right.$. DvsGasMf_[i][i] $)\left(w_{-}[\mathrm{i}][\mathrm{j}], \mathrm{w}_{-}[\mathrm{k}][\mathrm{j}]\right)$;

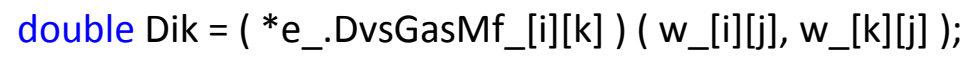

double Dki $=\left({ }^{*} e_{-}\right.$. DvsGasMf_[k][i] $)\left(w_{-}[\mathrm{i}][\mathrm{j}], \mathrm{w}_{-}[\mathrm{k}][\mathrm{j}]\right)$;

double Dkk $=\left({ }^{*} e_{-}\right.$. DvsGasMf_[k][k] $)\left(w_{-}[\mathrm{i}][j], w_{-}[k][j]\right)$;

double ddwi_Dii $=(*$ e_.dDvsGasMf_[i][i][i] $)\left(w_{-}[i][j], w \_[k][j]\right)$;

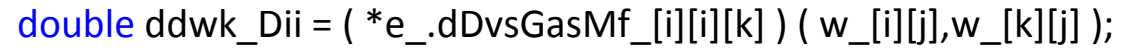

double ddwi_Dik $=\left({ }^{*} e_{-} . d D v s G a s M f \_[i][k][i]\right)\left(w_{-}[i][j], w_{-}[k][j]\right)$;

double ddwk_Dik $=\left({ }^{*}\right.$ e_dDvsGasMf_[i][k][k] $)\left(w_{-}[\mathrm{i}][\mathrm{j}], \mathrm{w}_{-}[\mathrm{k}][\mathrm{j}]\right)$;

double ddwi_Dki $=\left({ }^{*}\right.$ e_.dDvsGasMf_[k][i][i] $)\left(w_{-}[i][j], w_{-}[k][j]\right)$;

double ddwk_Dki $=\left({ }^{*}\right.$ e_dDvsGasMf_[k][i][k] $)\left(w_{-}[i][j], w_{-}[k][j]\right)$;

double ddwi_Dkk $=(*$ e_.dDvsGasMf_[k][k][i] $)\left(w_{-}[\mathrm{i}][\mathrm{j}], \mathrm{w}_{-}[\mathrm{k}][\mathrm{j}]\right)$;

double ddwk_Dkk $=\left({ }^{*} e_{-} . d D v s G a s M f \_[k][k][k]\right)\left(w_{-}[i][j], w_{-}[k][j]\right)$;

if $(\mathrm{j}<\mathrm{e}$ _.nZminus2_) $\{$ //for intermediate grid points

//@@state eqn

double Dkkki = Dkk*ddz_wk + Dki*ddz_wi;

double Diiik = Dii*ddz_wi + Dik*ddz_wk;

$d d t \_w \_[i][j]=(D k k k i+D i i i k) *\left(1-w \_[k][j]\right) / w 3 \_s q r * d d z \_w i$

$+($ Dkkki + Diiik $) * w \_[i][j] / w 3 \_s q r * d d z \_w k$

$+\left(D k i * w \_[i][j]+\left(1-w_{-}[k][j]\right) * D i i\right) / w 3 * d 2 d z 2 \_w i$

$+\left(D k k^{*} w_{-}[i][j]+\left(1-w_{-}[k][j]\right) * D k i\right) / w 3 * d 2 d z 2 \_w k$

$+\left(w \_[i][j]^{*}\left(d d w i \_D k k+d d w k \_D k i\right)+\left(1-w \_[k][j]\right)^{*}\left(d d w k \_D i i+d d w i \_D i k\right)\right) /$

w3*ddz_wi*ddz_wk

$+\left(w_{-}[i][j] * d d w i \_D k i+\left(1-w \_[k][j]\right) * d d w i \_D i i\right) / w 3 * d d z$ wi_sqr

$+\left(\left(1-w \_[k][j]\right) * d d w k \_D i k+w \_[i][j] * d d w k \_D k k\right) / w 3 * d d z \_w k \_s q r ;$ 


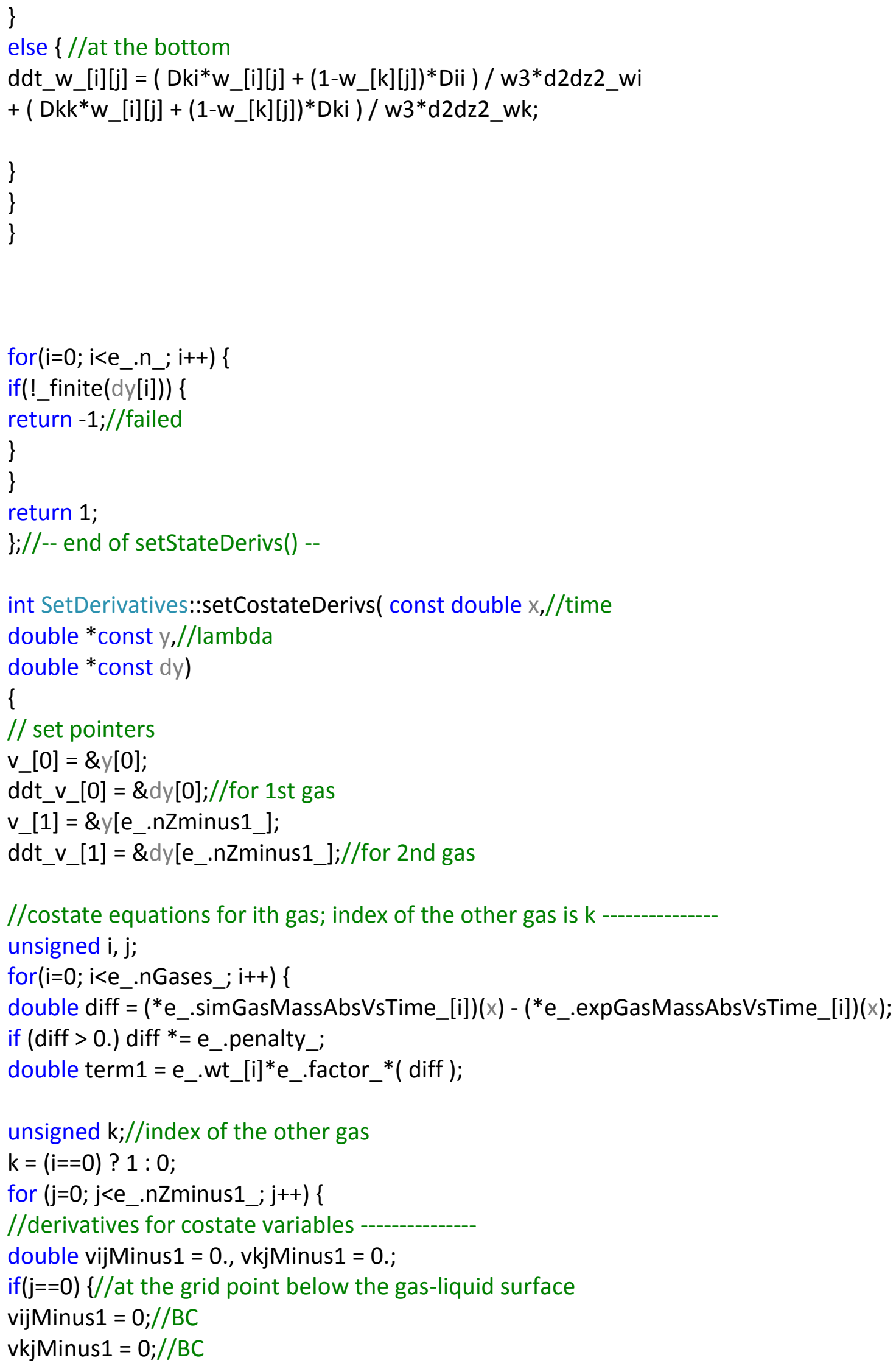




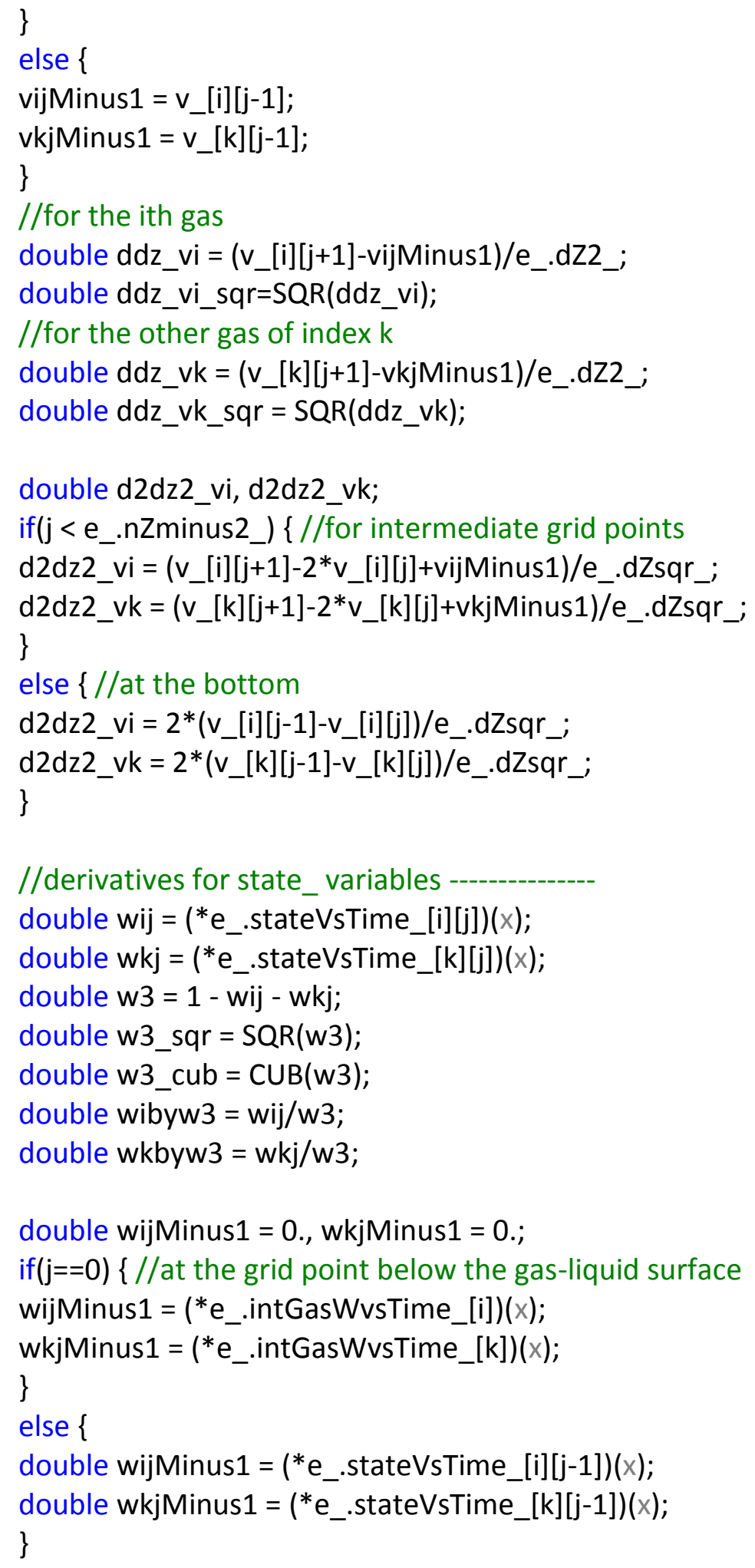

double ddz_wi, ddz_wi_sqr, ddz_wk, ddz_wk_sqr, d2dz2_wi, d2dz2_wk; if $(j<$ e_.nZminus2_) \{ //for intermediate grid points double wijPlus1 $=\left({ }^{*}\right.$ e_.stateVsTime_[i] $\left.[\mathrm{j}+1]\right)(\mathrm{x})$; 


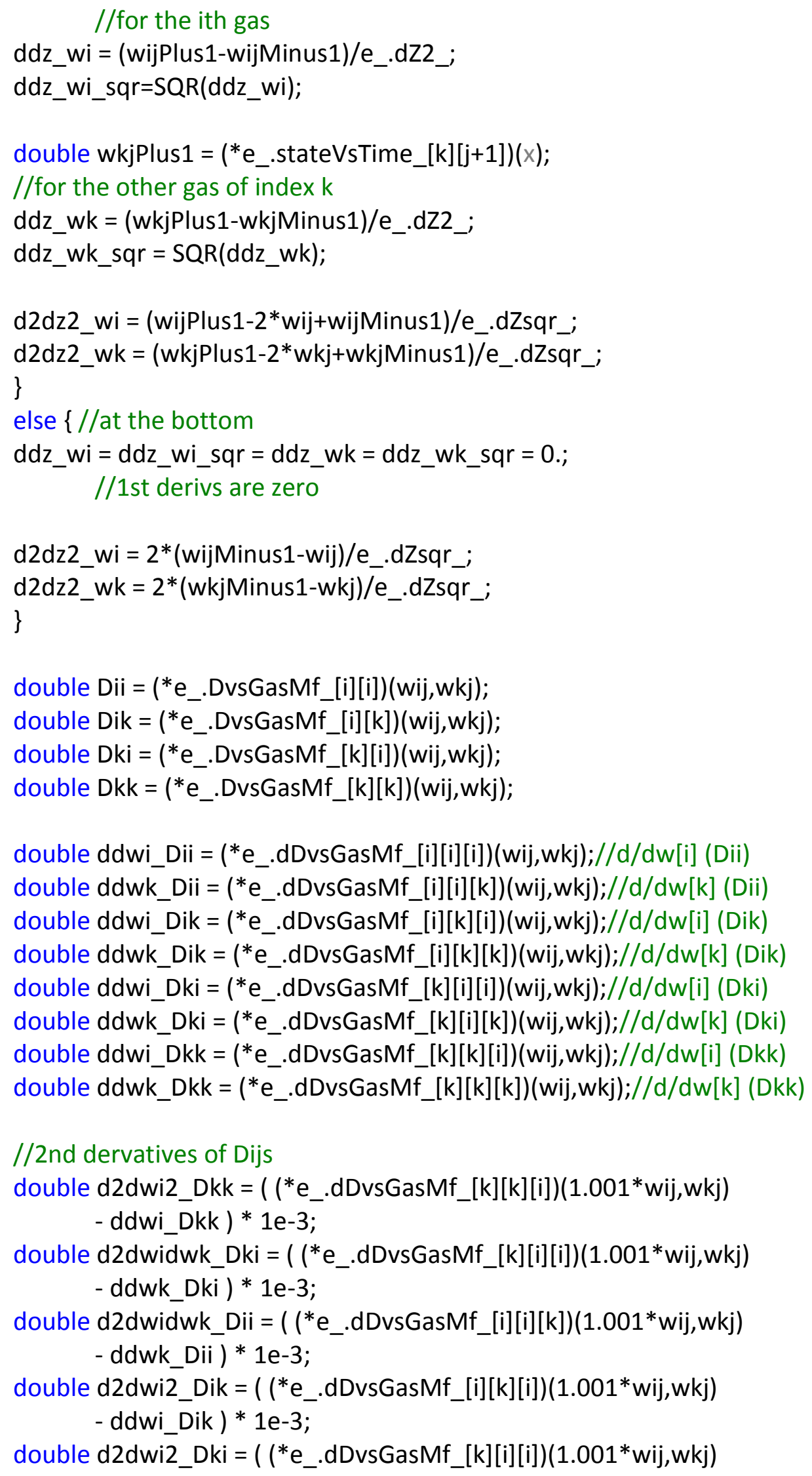


- ddwi_Dki )*1e-3;

double d2dwi2_Dii $=\left(\left({ }^{*} e_{-} . d D v s G a s M f \_[i][i][i]\right)(1.001 * w i j, w k j)\right.$

- ddwi_Dii ) *1e-3;

double d2dwidwk_Dik $=\left(\left(*^{*} e_{\text {. }}\right.\right.$ dDvsGasMf_[i][k][k])(1.001*wij,wkj)

- ddwk_Dik)*1e-3;

double d2dwidwk_Dkk $=\left(\left({ }^{*} e \_. d D v s G a s M f \_[k][k][k]\right)(w i j, w k j)\right.$

- ddwk_Dkk )*1e-3;

//p.6

double $D k k k i=D k k^{*} d d z \_w k+D k i * d d z \_w i ;$

double Diiik = Dii*ddz_wi + Dik*ddz_wk;

double ddz_Dkk $=$ ddwi_Dkk*ddz_wi + ddwk_Dkk*ddz_wk;

double ddz_Dki $=d d w i \_D k i * d d z \_w i+d d w k \_D k i * d d z \_w k ;$

double ddz_Dkkki $=d d z \_D k k^{*} d d z \_w k+D k k^{*} d 2 d z 2 \_w k+d d z \_D k i * d d z \_w i$

+ Dki*d2dz2_wi;

double ddz_Dii $=$ ddwi_Dii*ddz_wi + ddwk_Dii*ddz_wk;

double ddz_Dik=ddwi_Dik*ddz_wi + ddwk_Dik*ddz_wk;

double ddz_Diiik $=d d z \_D i{ }^{*} d d z \_w i+D i i * d 2 d z 2 \_w i+d d z \_D i k^{*} d d z \_w k$

+ Dik*d2dz2_wk;

double DwiDkkki = ddwi_Dkk*ddz_wk + ddwi_Dki*ddz_wi;

double DwiDiiik = ddwi_Dii*ddz_wi + ddwi_Dik*ddz_wk;

double ddwi_fi $=\left(2 *\left(1-w_{-}[k][j]\right) / w 3 *(D k k k i+D i i i k)+\left(1-w_{-}[k][j]\right) *(D w i D k k k i+\right.$ DwiDiiik $\left.)\right) /$

w3_sqr*ddz_wi

$+\left(\left(1+w_{-}[i][j]-w_{-}[k][j]\right) / w 3 * D k k k i+\left(1+w \_[i][j]-w \_[k][j]\right) / w 3 * D i i i k+w_{-}[i][j] * D w i D k k k i+\right.$

w_[i][j] ${ }^{*}$ DwiDiiik ) / w3_sqr*ddz_wk

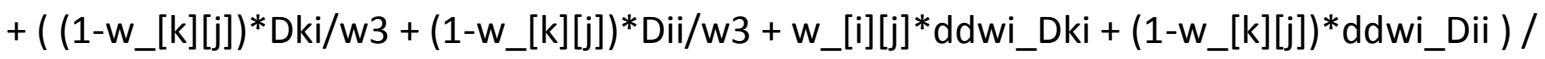

w3*d2dz2_wi

$+\left(\left(1-w_{-}[k][j]\right) * D k k / w 3+\left(1-w_{-}[k][j]\right) * D k i / w 3+w_{-}[i][j] * d d w i \_D k k+\left(1-w \_[k][j]\right) * d d w i \_D k i\right)$

/ w3*d2dz2_wk

$+\left(\left(1-w \_[k][j]\right) * d d w i \_D k k / w 3+\left(1-w \_[k][j]\right) * d d w k \_D k i / w 3+\left(1-w \_[k][j]\right) / w 3 * d d w k \_D i i+(1-\right.$

$\left.w_{-}[k][j]\right) * d d w i \_D i k / w 3$

$+w_{-}[i][j] * d 2 d w i 2 \_D k k+w_{-}[i][j] * d 2 d w i d w k \_D k i+\left(1-w \_[k][j]\right) * d 2 d w i d w k \_D i i+(1-$

$\left.\left.w_{-}[k][j]\right) * d 2 d w i 2 \_D i k\right) / w 3 * d d z \_w i * d d z \_w k$

$+\left(\left(1-w \_[k][j]\right) * d d w i \_D k i / w 3+\left(1-w \_[k][j]\right) * d d w i \_D i i / w 3+w \_[i][j] * d 2 d w i 2 \_D k i+(1-\right.$

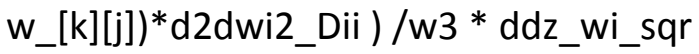

$+\left(\left(1-w \_[k][j]\right) * d d w k \_D i k / w 3+\left(1-w \_[k][j]\right) * d d w k \_D k k / w 3+\left(1-w \_[k][j]\right) * d 2 d w i d w k \_D i k+\right.$

w_[i][j]*d2dwidwk_Dkk ) /w3 * ddz_wk_sqr; //p.6

double ddwi_fk $=\left(-1 / w 3 \_s q r+\left(1-w \_[i][j]\right) / w 3 \_c u b\right) *$ Diiik*ddz_wk + (1-

w_[i][j])/w3_sqr*DwiDiiik*ddz_wk 
$+\left(-1 /\right.$ w3_sqr $\left.+\left(1-w_{-}[i][j]\right) / w 3 \_c u b\right) * D k k k i * d d z \_w k+(1-$

w_[i][j])/w3_sqr*DwiDkkki*ddz_wk

+ w_[k][j]/w3_cub*Diiik*ddz_wi + w_[k][j]/w3_sqr*DwiDiiik*ddz_wi

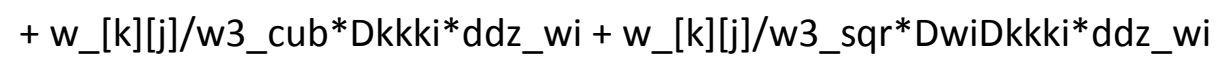

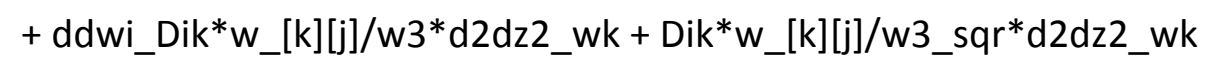

+ ddwi_Dkk*(1-w_[i][j] $) / w 3 * d 2 d z 2 \_w k+D k k^{*}\left(-1 / w 3+\left(1-w \_[i][j]\right) / w 3 \_s q r\right) * d 2 d z 2 \_w k$

+ddwi_Dii*w_[k][j]/w3*d2dz2_wi + Dii*w_[k][j]/w3_sqr*d2dz2_wi

+ddwi_Dik*(1-w_[i][j])/w3*d2dz2_wi + Dik*(-1/w3 + (1-w_[i][j])/w3_sqr)*d2dz2_wi

$+\left(w_{-}[k][j] / w 3 \_s q r * d d w k \_D i i+w_{-}[k][j] / w 3 * d 2 d w i d w k \_D i i\right) * d d z \_w i^{*} d d z \_w k$

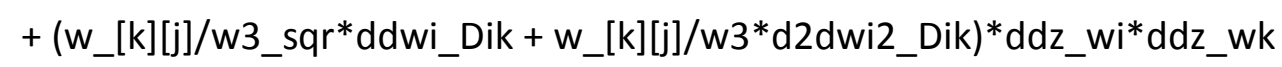

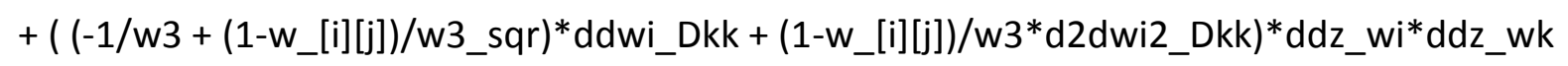

$+\left(\left(-1 / w 3+\left(1-w_{-}[i][j]\right) / w 3 \_s q r\right) * d d w k \_D k i+(1-\right.$

w_[i][j])/w3*d2dwidwk_Dki)*ddz_wi*ddz_wk

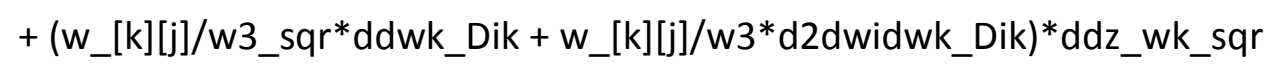

$+\left(\left(-1 / w 3+\left(1-w \_[i][j]\right) / w 3 \_s q r\right) * d d w k \_D k k+\left(1-w \_[i][j]\right) / w 3 * d 2 d w i d w k \_D k k\right) * d d z \_w k \_s q r$

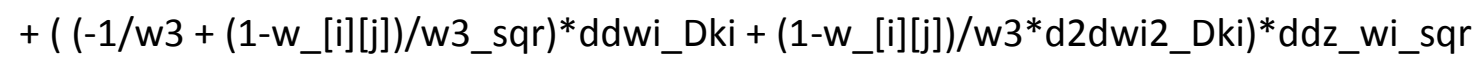

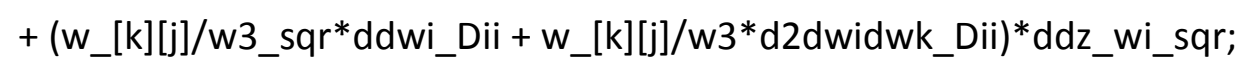

double ddwiz_fi $=\left(1-w_{-}[k][j]\right) *(D k k k i+$ Diiik + Dki + Dii $) / w 3 \_s q r * d d z \_w i$

$+w_{-}[i][j] *(D k i+D i i) / w 3 \_s q r * d d z \_w k$

$+\left(w_{-}[i][j] *\left(d d w i \_D k k+d d w k \_D k i\right)+\left(1-w \_[k][j]\right) *\left(d d w k \_D i i+d d w i \_D i k\right)\right) / w 3 * d d z \_w k$ $+2 *\left(w_{-}[i][j] * d d w i \_D k i+\left(1-w \_[k][j]\right) * d d w i \_D i i\right) / w 3 * d d z \_w i ; / / p .7$

double d2dzdwi_Dkk = d2dwi2_Dkk*ddz_wi + d2dwidwk_Dkk*ddz_wk;

double d2dwk2_Dki $=\left(\left({ }^{*}\right.\right.$ _.dDvsGasMf_[k][i] $\left.[k]\right)(w i j, 1.003 * w k j)-$ ddwk_Dki $) * 1 e-3$;

double d2dwk2_Dik $=\left(\left({ }^{*}\right.\right.$ e_dDvsGasMf_[i][k][k])(wij,1.003*wkj) - ddwk_Dik $) * 1 e-3$;

double d2dzdwk_Dki $=$ d2dwk2_Dki*ddz_wk + d2dwidwk_Dki*ddz_wi;

double d2dzdwk_Dik = d2dwk2_Dik*ddz_wk + d2dwidwk_Dik*ddz_wi; 
double d2dwk2_Dii $=\left(\left({ }^{*}\right.\right.$ e_dDvsGasMf_[i][i][k])(wij,1.003*wkj) - ddwk_Dii $) * 1 e-3$;

double d2dzdwk_Dii = d2dwk2_Dii*ddz_wk + d2dwidwk_Dii*ddz_wi;

double d2dzdwi_Dik = d2dwi2_Dik*ddz_wi + d2dwidwk_Dik*ddz_wk;

double d2dzdwi_Dki $=$ d2dwi2_Dki*ddz_wi + d2dwidwk_Dki*ddz_wk;

double d2dzdwi_Dii = d2dwi2_Dki*ddz_wi + d2dwidwk_Dii*ddz_wk;

double d2dzdwiz_fi $=-d d z \_w k^{*}($ Dkkki + Diiik + Dki + Dii) $/$ w3_sqr*ddz_wi

$+\left(1-w_{-}[k][j]\right) *\left(d d z \_D k k k i+d d z \_D i i i k+d d z \_D k i+d d z \_D i i\right) /$ w3_sqr*ddz_wi

$+\left(1-w_{-}[\mathrm{k}][j]\right) *($ Dkkki + Diiik + Dki + Dii $) * 2 * w 3 *\left(d d z \_w i+d d z \_w k\right) / w 3 \_c u b * d d z \_w i$

$+\left(1-w_{-}[k][j]\right) *(D k k k i+D i i i k+D k i+D i i) / w 3 \_s q r * d 2 d z 2 \_w i$

$+d d z \_w i^{*}(D k i+D i i) / w 3 \_s q r * d d z \_w k+w_{-}[i][j] *\left(d d z \_D k i+d d z \_D i i\right) / w 3 \_s q r * d d z \_w k$

$+w_{-}[\mathrm{i}][\mathrm{j}] *(\mathrm{Dki}+\mathrm{Dii}) * 2 * w 3 *\left(\mathrm{ddz} \_w i+d d z_{-} w k\right) /$ w3_cub *ddz_wk

$+w_{-}[i][j] *(D k i+D i i) / w 3 \_s q r * d 2 d z 2 \_w k$

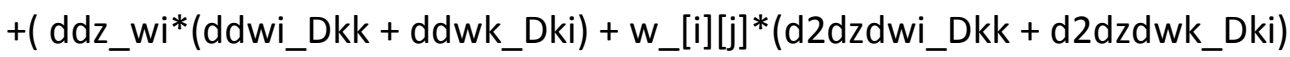

$\left.-d d z \_w k^{*}\left(d d w k \_D i i+d d w i \_D i k\right)+\left(1-w \_[k][j]\right) *\left(d 2 d z d w k \_D i i+d 2 d z d w i \_D i k\right)\right) / w 3 * d d z \_w k$

$+\left(w_{-}[i][j] *\left(d d w i \_D k k+d d w k \_D k i\right)+\left(1-w \_[k][j]\right) *\left(d d w k \_D i i+d d w i \_D i k\right)\right) *$

$2 * w 3 *\left(d d z \_w i+d d z \_w k\right) / w 3 \_c u b * d d z \_w k$

$+\left(w_{-}[i][j] *\left(d d w i \_D k k+d d w k \_D k i\right)+\left(1-w \_[k][j]\right) *\left(d d w k \_D i i+d d w i \_D i k\right)\right) / w 3 * d 2 d z 2 \_w k$

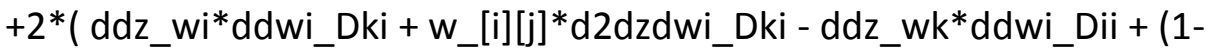

w_[k][j])*d2dzdwi_Dii )/w3*ddz_wi

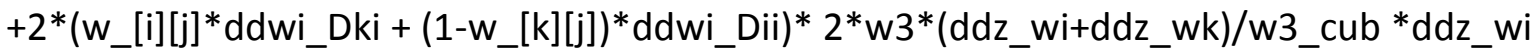
$+2 *\left(w_{-}[i][j] * d d w i \_D k i+\left(1-w \_[k][j]\right) * d d w i \_D i i\right) / w 3 * d 2 d z 2 \_w i ;$

double ddwizz_fi $=\left(\right.$ Dki $^{*} w_{-}[i][j]+\left(1-w_{-}[k][j]\right) *$ Dii $) / w 3 ; / / p .11$

double d2dzdwizz_fi $=\left(w_{-}[i][j] * d d z \_D k i+\left(1-w_{-}[k][j]\right) * d d z \_D i i+\left(\left(1-w_{-}[k][j]\right) / w 3 * d d z \_w i+\right.\right.$ $\left.w_{-}[i][j] / w 3 * d d z \_w k\right) * D k i+$

$\left.\left(2 *\left(1-w_{-}[k][j]\right) / w 3 * d d z \_w i+d d z \_w k\right) * D i i\right) / w 3 ; / / p .15$

double d2dz2_Dki = ddwi_Dki*d2dz2_wi + d2dzdwi_Dki*ddz_wi + ddwk_Dki*d2dz2_wk + d2dzdwk_Dki*ddz_wk;

double d2dz2_Dii = ddwi_Dii*d2dz2_wi + d2dzdwi_Dii*ddz_wi + ddwk_Dii*d2dz2_wk + d2dzdwk_Dii*ddz_wk; 
double d2dz2_Dik = ddwi_Dik*d2dz2_wi + d2dzdwi_Dik*ddz_wi + ddwk_Dik*d2dz2_wk + d2dzdwk_Dik*ddz_wk;

double d3dz2dwizz_fi $=\left(d d z \_w i * d d z \_D k i+w_{-}[i][j] * d 2 d z 2 \_D k i\right.$

$-d d z \_w k^{*} d d z \_D i i+\left(1-w \_[k][j]\right) * d 2 d z 2 \_D i i$

$+\left(\left(1-w \_[k][j]\right) / w 3 * d d z \_w i+w_{-}[i][j] / w 3 * d d z \_w k\right) * d d z \_D k i+$

$+\left(\left(1-w \_[k][j]\right) / w 3 \_s q r * d d z \_w i+\left(-1 / w 3+\left(1-w \_[k][j]\right) / w 3 \_s q r\right) * d d z \_w k+(1-\right.$

$\left.w_{-}[\mathrm{k}][\mathrm{j}]\right) / \mathrm{w} 3^{*} \mathrm{~d} 2 \mathrm{dz2} \_w i$

$+\left(1 / w 3+w \_[i][j] / w 3 \_s q r\right) * d d z \_w i+w \_[i][j] / w 3 \_s q r * d d z \_w k+w \_[i][j] / w 3 * d 2 d z 2 \_w k$

)$^{*} \mathrm{Dki}$

$+\left(\left(1-w \_[k][j]\right) / w 3 * d d z \_w i+w_{-}[i][j] / w 3 * d d z \_w k\right) * d d z \_D k i$

$+\left(2 *\left(\left(1-w \_[k][j]\right) / w 3 \_s q r * d d z \_w i+\left(-1 / w 3+\left(1-w \_[k][j]\right) / w 3 \_s q r\right) * d d z \_w k\right) * d d z \_w i\right.$

$\left.+2 *\left(1-w \_[k][j]\right) / w 3 * d 2 d z 2 \_w i+d 2 d z 2 \_w k\right) * D i i$

$\left.+\left(2 *\left(1-w \_[k][j]\right) / w 3 * d d z \_w i+d d z \_w k\right) * d d z \_D i i\right) / w 3+$

+ d2dzdwizz_fi/w3*(ddz_wi+ddz_wk);

double ddwiz_fk $=\left(1-w \_[i][j]\right) / w 3 \_s q r * D i i * d d z \_w k+\left(1-w \_[i][j]\right) / w 3 \_s q r * D k i * d d z \_w k$

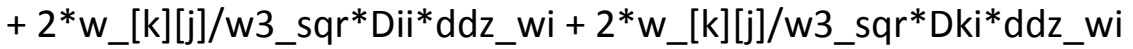

$+w \_[k][j] / w 3 * d d w k \_D i i * d d z \_w k+w \_[k][j] / w 3 * d d w i \_D i k^{*} d d z \_w k$

$+\left(1-w \_[i][j]\right) / w 3 * d d w i \_D k k^{*} d d z \_w k+\left(1-w \_[i][j]\right) / w 3 * d d w k \_D k i * d d z \_w k$

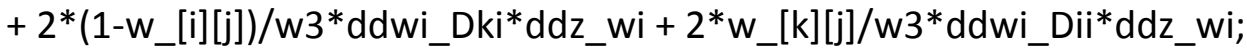

$/ /$ double ddwiz_fk $=\left(1-w \_[i][j]\right) / w 3 \_s q r^{*}\left(D i i^{*} d d z \_w k+D k i * d d z \_w k\right)$

$/ /+2 * w \_[k][j] / w 3 \_s q r^{*}\left(D i i * d d z \_w i+D k i * d d z \_w i\right)$

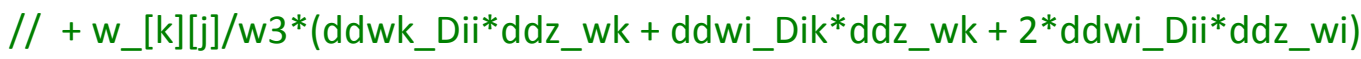

$/ /+\left(1-w \_[i][j]\right) / w 3^{*}\left(d d w i \_D k k^{*} d d z \_w k+d d w k \_D k i^{*} d d z \_w k+2 * d d w i \_D k i * d d z \_w i\right) ;$

double d2dzdwiz_fk $=\left(\left(1-w \_[i][j]+w \_[k][j]\right) / w 3 \_c u b * d d z \_w i+2 *(1-\right.$

w_[i][j])/w3_cub*ddz_wk)*(Dii*ddz_wk + Dki*ddz_wk)

$+\left(1-w \_[i][j]\right) / w 3 \_s q r *\left(d d z \_D i i * d d z \_w k+D i i * d 2 d z 2 \_w k+d d z \_D k i * d d z \_w k+\right.$ Dki*d2dz2_wk)

$+2 *\left(2 * w \_[k][j] / w 3 \_c u b * d d z \_w i+\left(1-w \_[i][j]+w_{-}[k][j]\right) / w 3 \_c u b * d d z \_w k\right) *\left(D i i * d d z \_w i+\right.$ Dki*ddz_wi)

$+2 * w \_[k][j] / w 3 \_s q r *\left(d d z \_D i i * d d z \_w i+D i i * d 2 d z 2 \_w i+d d z \_D k i * d d z \_w i+D k i * d 2 d z 2 \_w i\right)$

$+\left(w \_[k][j] / w 3 \_s q r * d d z \_w i+\left(1-w \_[i][j]\right) / w 3 \_s q r * d d z \_w k\right) *\left(d d w k \_D i i * d d z \_w k+\right.$ ddwi_Dik*ddz_wk + 2*ddwi_Dii*ddz_wi)

$+w_{-}[\mathrm{k}][j] / w 3^{*}\left(d 2 d z d w k \_D i{ }^{*} d d z \_w k+d d w k \_D i{ }^{*} d 2 d z 2 \_w k+d 2 d z d w i \_D i k * d d z \_w k+\right.$ ddwi_Dik*d2dz2_wk 
$\left.+2 * d 2 d z d w i \_D i i * d d z \_w i+2 * d d w i \_D i i * d 2 d z 2 \_w i\right)$

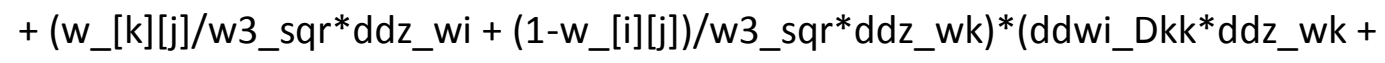
ddwk_Dki*ddz_wk + 2*ddwi_Dki*ddz_wi)

$+\left(1-w_{-}[i][j]\right) / w 3^{*}\left(d 2 d z d w i \_D k k^{*} d d z \_w k+d d w i \_D k k^{*} d 2 d z 2 \_w k+d 2 d z d w k \_D k i^{*} d d z \_w k+\right.$ ddwk_Dki*d2dz2_wk

$\left.+2 * d 2 d z d w i \_D k i * d d z \_w i+2 * d d w i \_D k i * d 2 d z 2 \_w i\right)$;

double ddwizz_fk $=\left(\right.$ Dii*w_[k][j] + Dik* $\left.{ }^{*}\left(1-w \_[i][j]\right)\right) / w 3$;

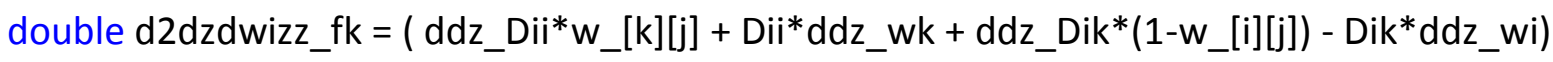
/w3 + ddwizz_fk/w3*(ddz_wi + ddz_wk);

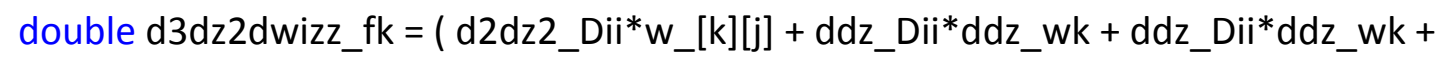
Dii*d2dz2_wk

+d2dz2_Dik*(1-w_[i][j]) - ddz_Dik*ddz_wi - ddz_Dik*ddz_wi - Dik*d2dz2_wi ) /w3

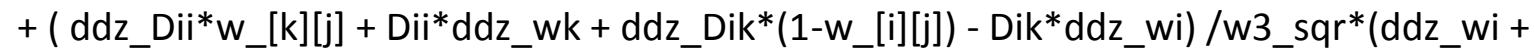
ddz_wk)

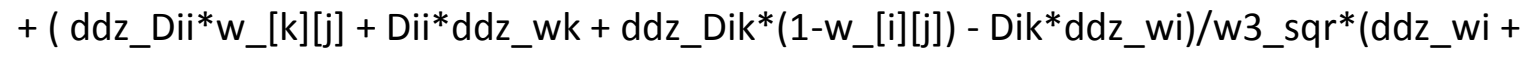
ddz_wk)

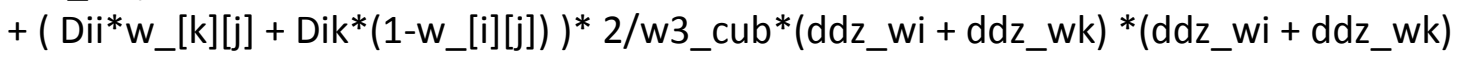

$+($ Dii*w_[k][j] + Dik*(1-w_[i][j]) )/w3_sqr*(d2dz2_wi + d2dz2_wk);

//@@check term1

if $\left(j<e_{-} . n Z m i n u s 2 \_\right)\{/ /$for intermediate grid points

ddt_v_[i][j] = term1 - v_[i][j]*ddwi_fi $+d d z_{-} v i * d d w i z f i+v_{-}[i][j] * d 2 d z d w i z f i$

- d2dz2_vi*ddwizz_fi - 2*ddz_vi*d2dzdwizz_fi - v_[i][j]*d3dz2dwizz_fi

$+v_{-}[k][j] * d d w i \_f k-d d z \_v k * d d w i z \_f k-v_{-}[k][j] * d 2 d z d w i z \_f k$

$+d 2 d z 2 \_v k^{*} d d w i z z \_f k+2 * d d z \_v{ }^{*} d 2 d z d w i z z \_f k+v_{-}[k][j] * d 3 d z 2 d w i z z \_f k ;$

\}

else $\{/ /$ at the bottom

ddt_v_[i][j] = term1 - v_[i][j]*ddwi_fi + v_[i][j]*d2dzdwiz_fi

- d2dz2_vi*ddwizz_fi - v_[i][j]*d3dz2dwizz_fi

$+v_{-}[k][j] * d d w i f k-d d z \_v k * d d w i z \_f k-v_{-}[k][j] * d 2 d z d w i z \_f k$

$+d 2 d z 2 \_v k * d d w i z z+f k+v_{-}[k][j] * d 3 d z 2 d w i z z+f k$; 


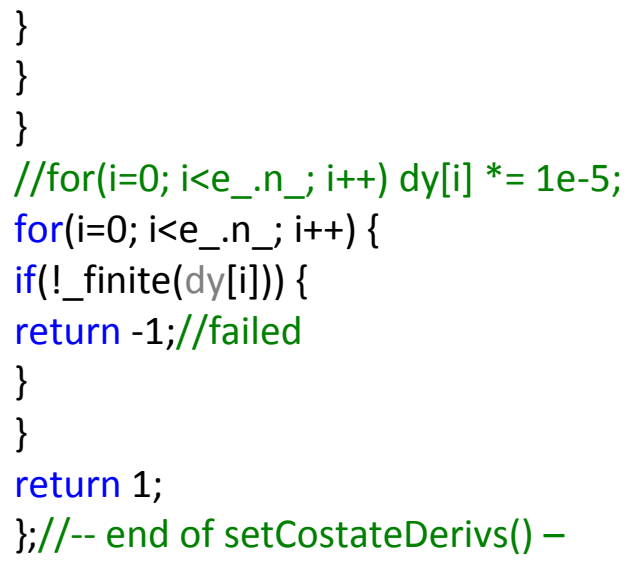

\section{Set Derives. $\mathrm{h}$}

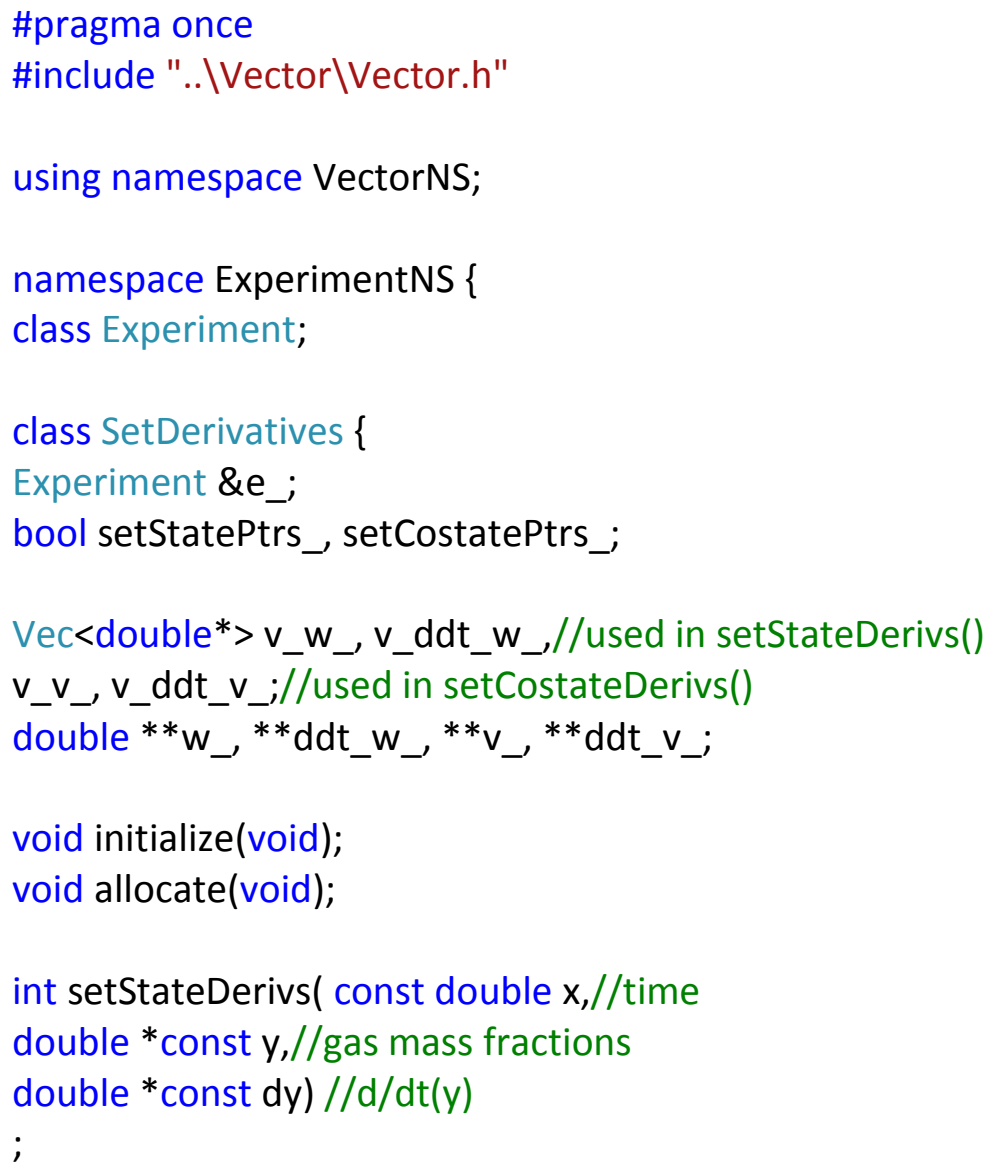


int setCostateDerivs( const double $\mathrm{x}, / /$ time

double * const $\mathrm{y}, / /$ lambda

double * const dy)

;//-- end of setCostateDerivs() --

public:

SetDerivatives(Experiment \&e);

SetDerivatives(void);

void set(void);

//------ function called by de object [as SetDerivs() ]

const int operator () (const double $x$, double * const $y$, double * const dy);

\};//--- end of class SetDerivatives ---

\}// --- end of ExperimentNS ---

\section{Get Gradient.Cpp}

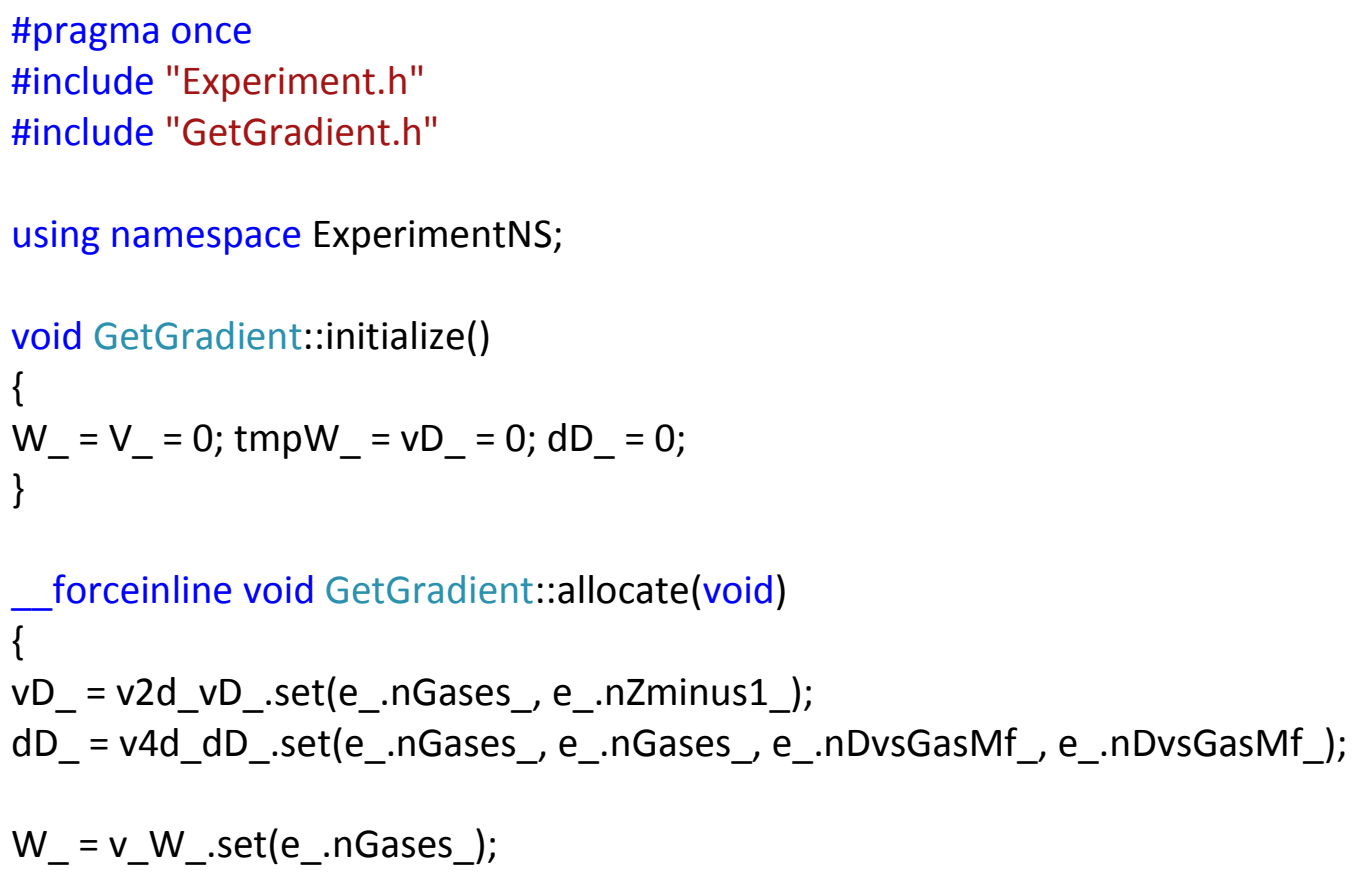




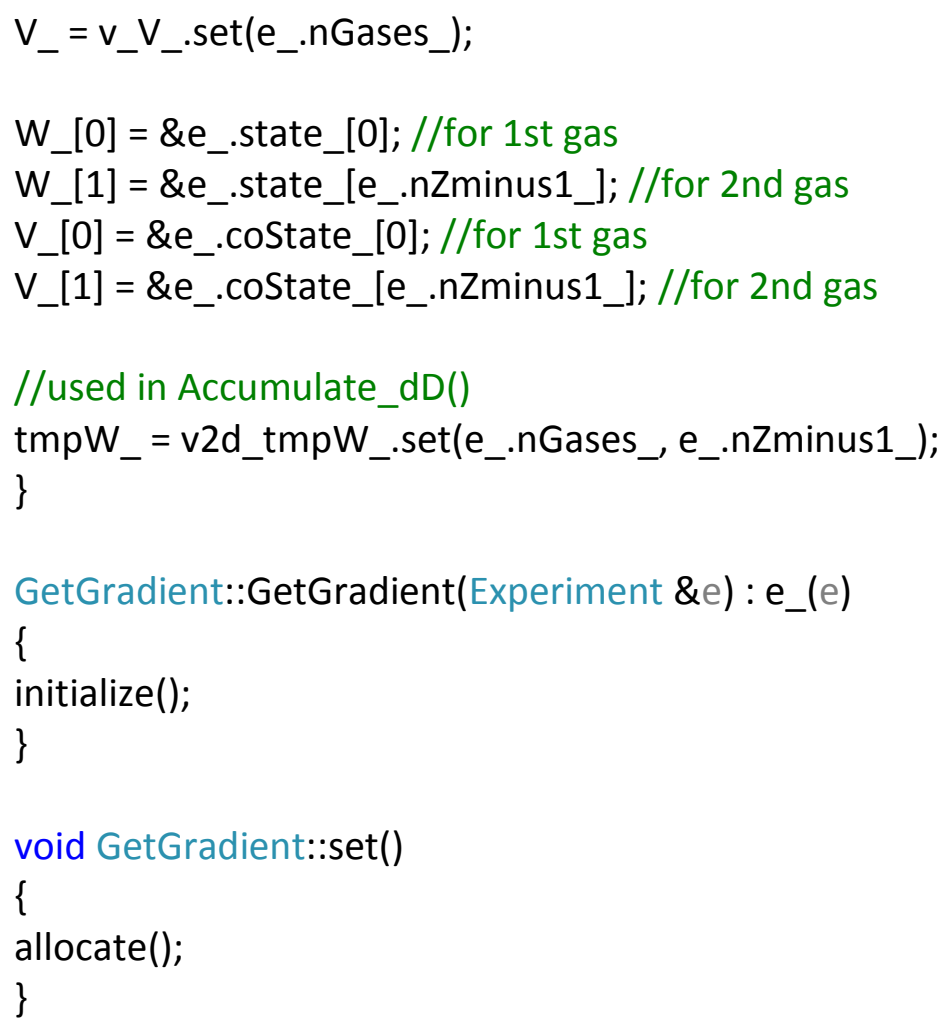




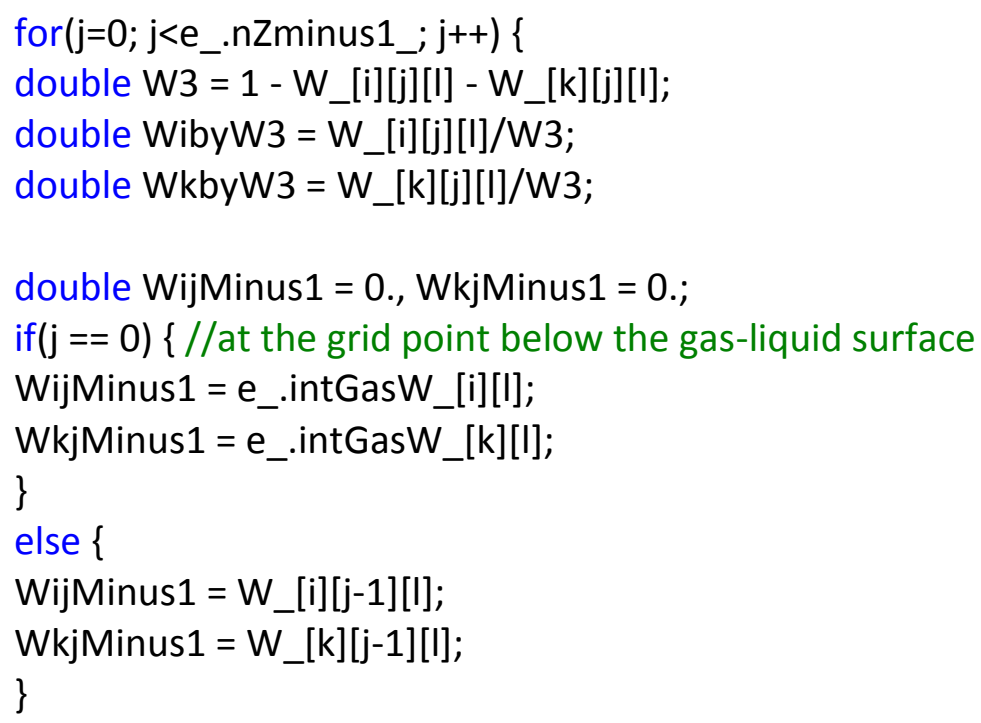

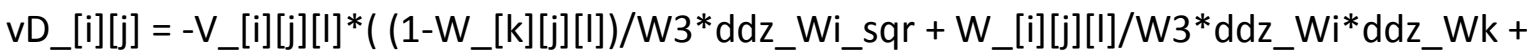
$\left(1-W_{-}[k][j][l]\right) * d 2 d z 2 \_W i / / d d D 00 \_f 1$

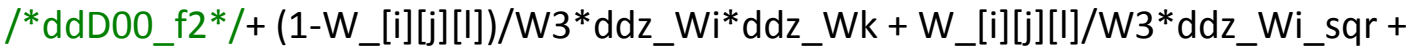
W_[k][j][l]/W3*d2dz2_Wi ) /W3;

//coeff of delta D01 (and delta D10 in the next outermost iteration)

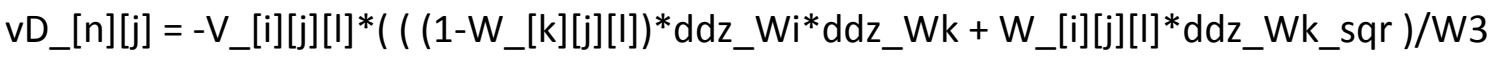
//ddD01_f1 


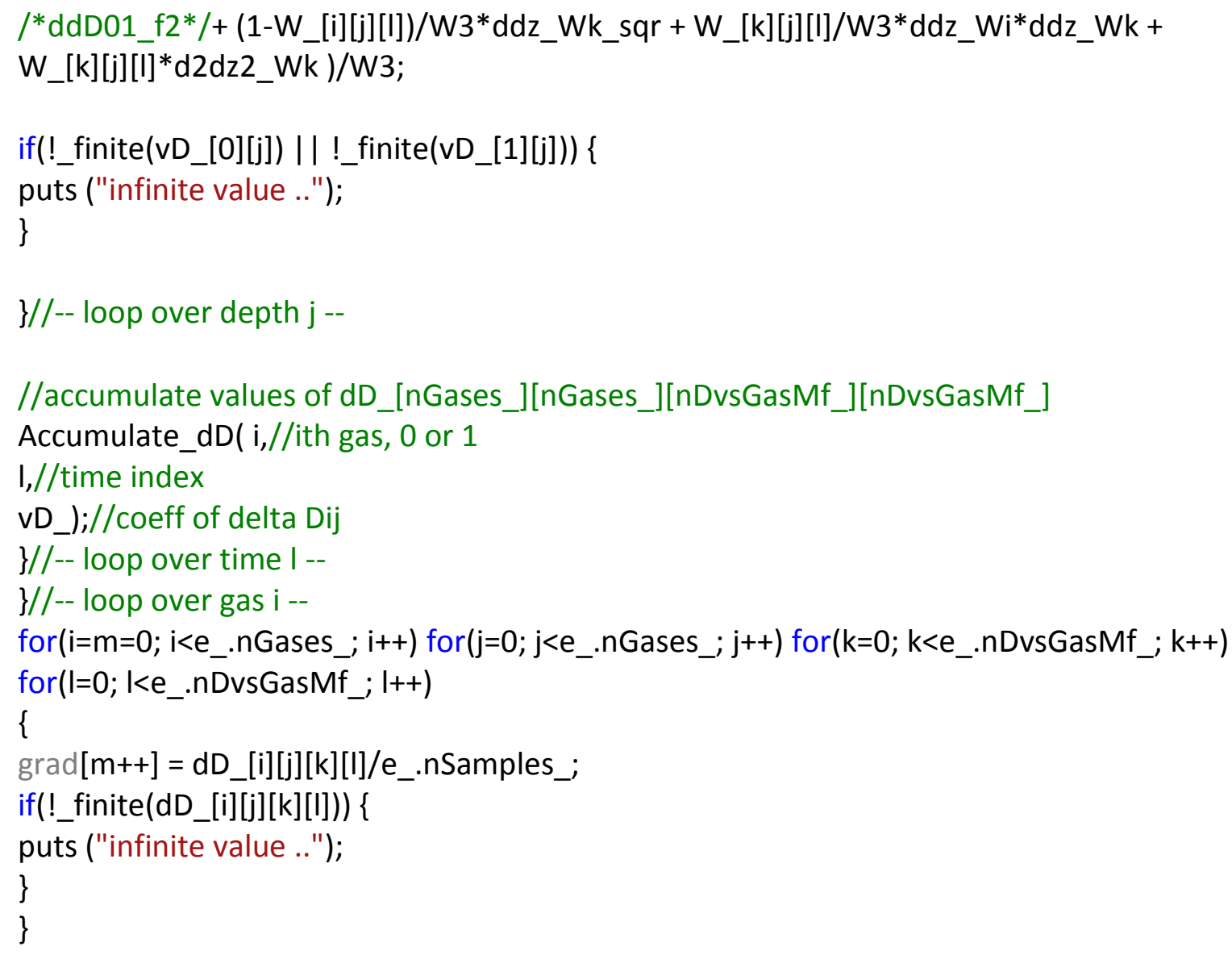


//interpolate or extrapolate at each grid point on the base of the 3d graph dD_vs W0 and W1

for $\left(k=0 ; k<e \_. n D v s G a s M f \_; k++\right)\{$

for $(\mathrm{l}=0 ; \mathrm{k}$ e_.nDvsGasMf_; $\mathrm{l++})\{$

double val = dDvsWOW1_( e_.p_gasMf_[0][k], e_.p_gasMf_[1][I] );

if(!_finite(val)) \{

puts ("infinite value ..");

\}

dD_[i][j][k][I]+=( val );

\}

\}

\}

\}

\section{Get Gradient .h}

\#pragma once

\#include "..IVector VVector.h"

\#include "..\Spline\Krig.h"

using namespace VectorNS;

using namespace KrigNS;

namespace ExperimentNS \{

class Experiment;

Aim: provide getGrad() while maintaining the state of some variables during iterations

Usage: GetGradient getGrad; getGrad();//uses overloaded operator()

Comments:

1. friend class of Experiment 
class GetGradient \{

Experiment \&e_;

Vec $<$ double** $>$ v_W_, v_V_;//used in getGrad()

double $* * * \mathrm{~W}_{\text {, }} * * * \mathrm{~V}_{-}$;

Vec2d<double $>$ v2d_tmpW_,//used in Accumulate_dD()

v2d_vD_;//used in $\operatorname{getGrad}()$

double $* *$ tmpW_, ** vD_;

Vec4d $<$ double> v4d_dD_///nGases_][nGases_][nDvsGasMf_][nDvsGasMf_]

double $* * * * d D \_/ /$used in $\operatorname{getGrad}()$

Krig dDvsWOW1_;

void initialize(void);

void allocate(void);

public:

GetGradient(Experiment \&e);

void set(void);

GetGradient(void);

//returns whether the call was successful; gradients are returned in grad[]

const bool operator() (double $* * * *$ const $\mathrm{D}, / / \mathrm{IN}$ : optimization parameter ie diffusivity double *const grad)//OUT: gradient

;

//called by getGrad() to set dD_[i][0][nDvsGasMf_][nDvsGasMf_]

void Accumulate_dD( const unsigned $\mathrm{i}$,//row index of the $D$ matrix

const unsigned $\mathrm{nT}_{-} / / /$time instant

double **const vD)//vD[nGases_][nSamples_], e.g. vD_00 and vD_01

;

\};//--- end of class GetGradient ---

3// --- end of ExperimentNS --- 
Appendix D 
Plots in the following pages are the diffusivity values that were obtained in the sensitivity analysis when initial diffusivity estimate was increased and decreased by $\pm 3 \%$.

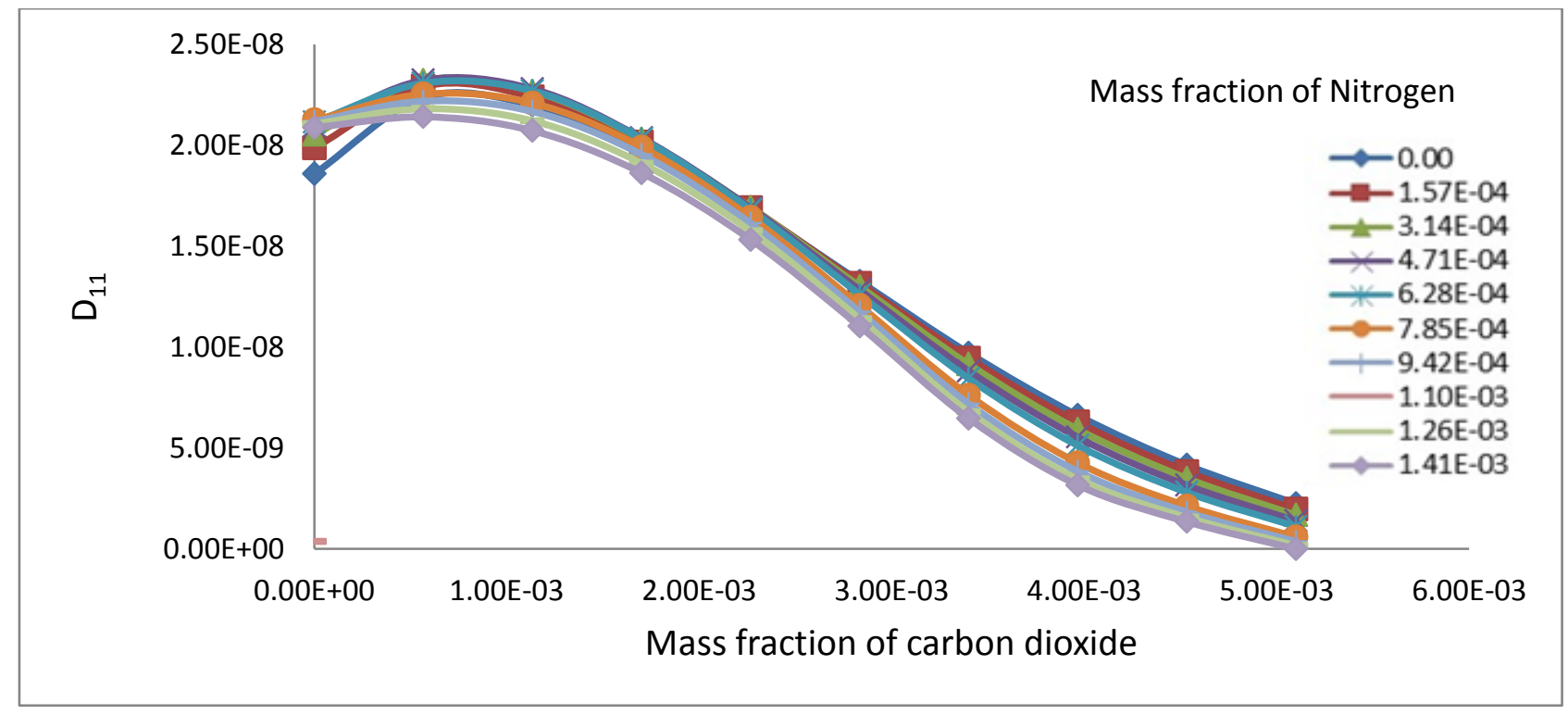

D 1 Main diffusion coefficient D11 with $+3 \%$ increase in initial diffusivity estimate

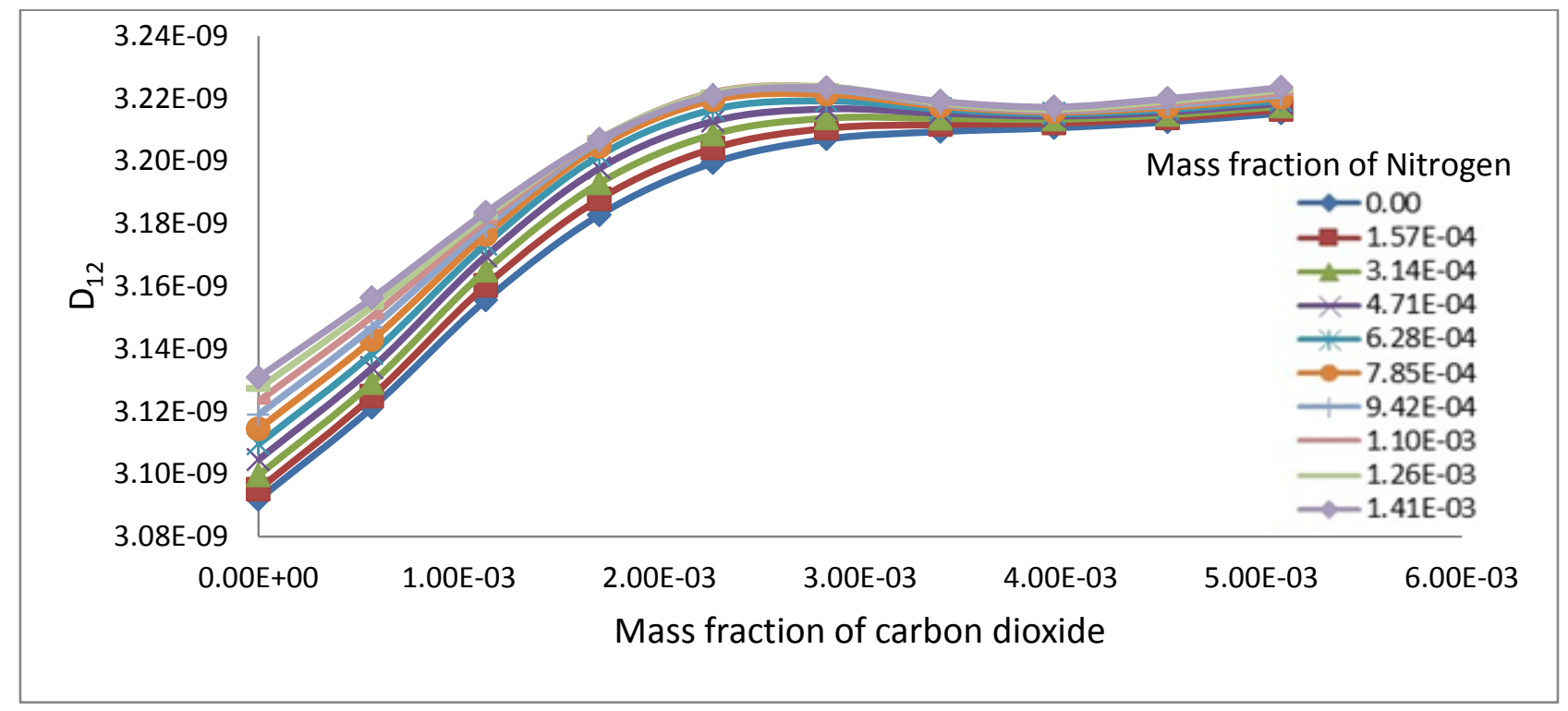

D 2 Cross diffusion coefficient D12 with $+3 \%$ increase in initial diffusivity estimate 


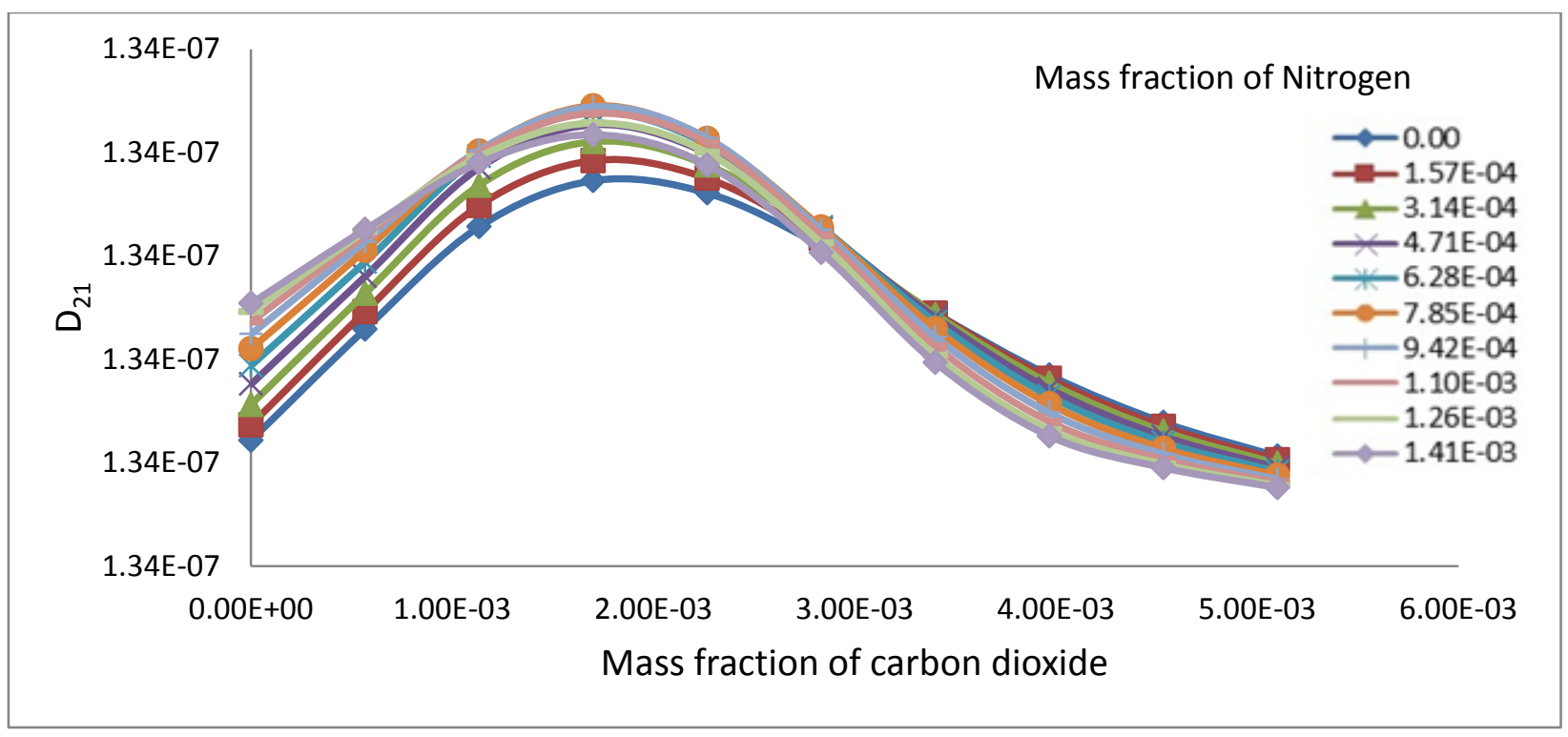

D 3 Cross diffusion coefficient D21 with $+3 \%$ increase in initial diffusivity estimate

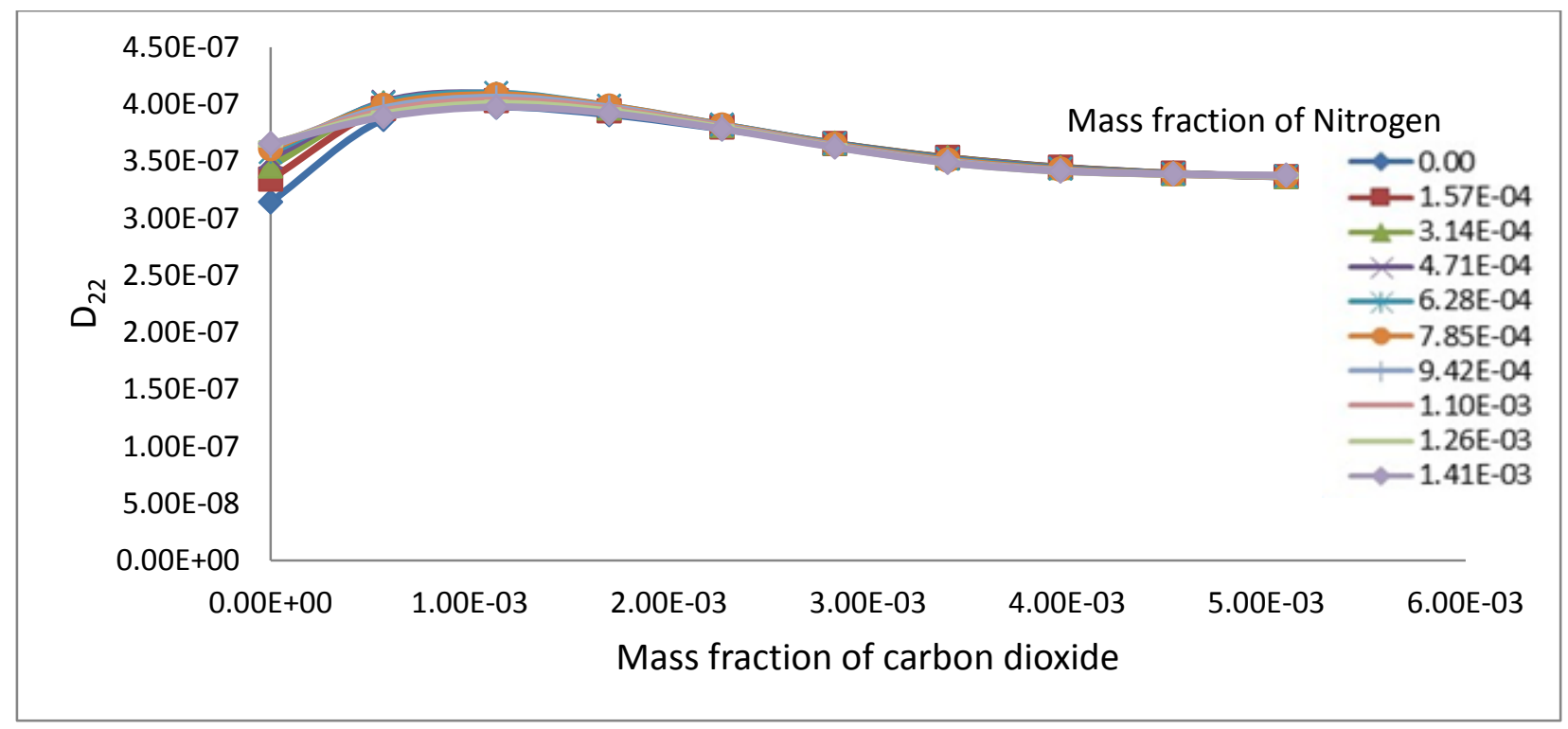

D 4 Main diffusion coefficient D22 with $+3 \%$ increase in initial diffusivity estimate 


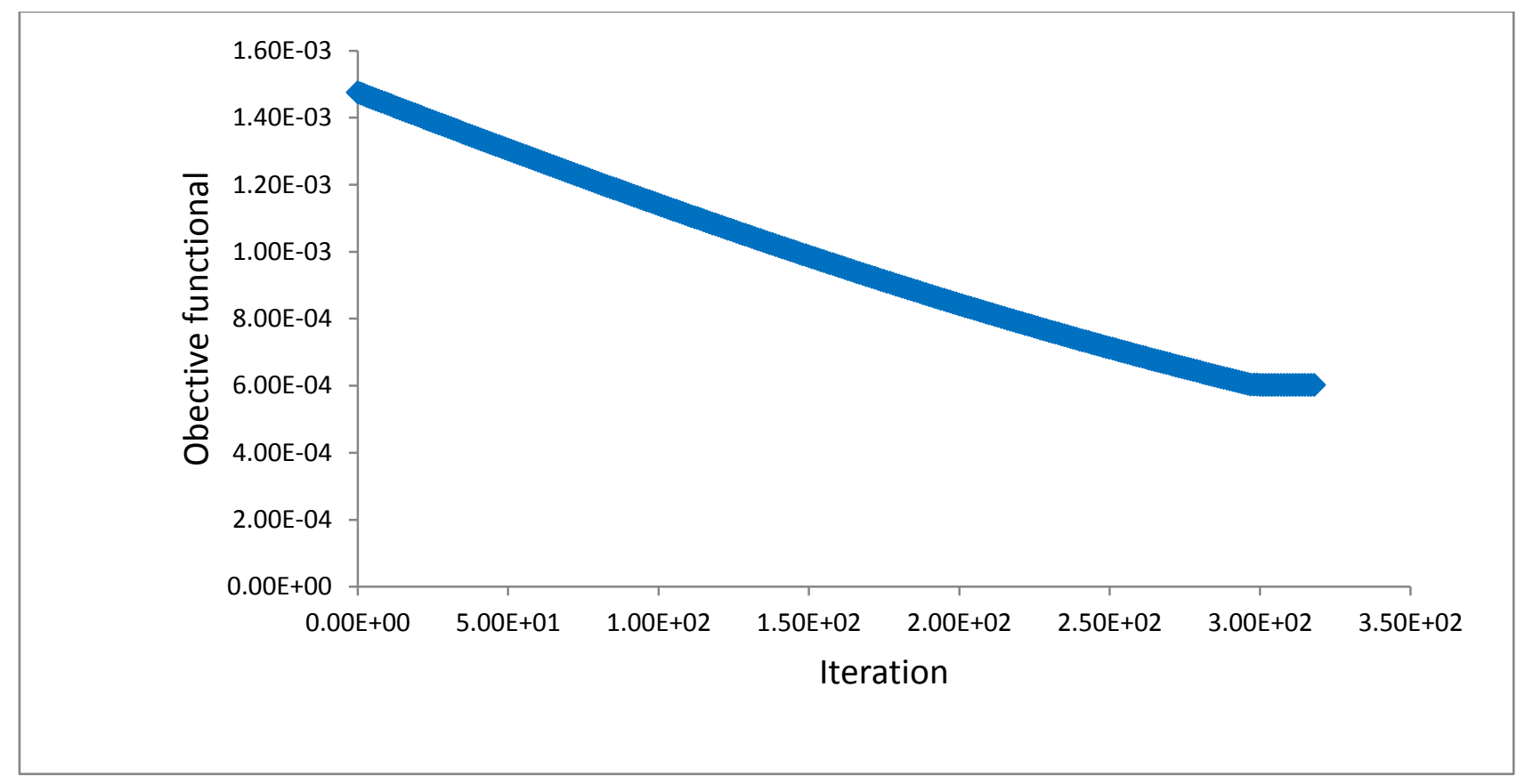

D 5 The convergence of objective functional to the optimum with the $+3 \%$ increase in the initial diffusivity estimate

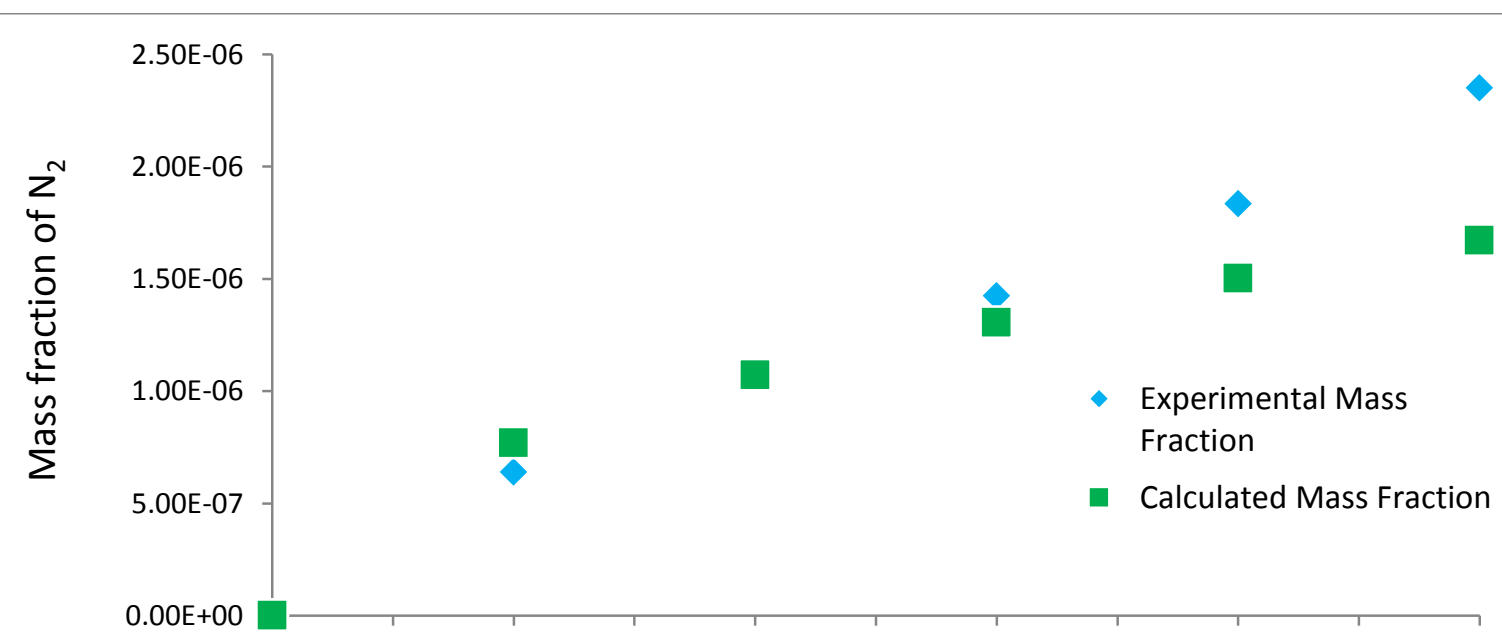

0.00E+00 1.00E-01 2.00E-01 3.00E-01 4.00E-01 5.00E-01 6.00E-01 7.00E-01 8.00E-01 9.00E-01 1.00E+00

Time in minutes

D 6 Experimental versus calculated gas mass of nitrogen absorbed in polystyrene with the $+3 \%$ increase in the initial diffusivity estimate 


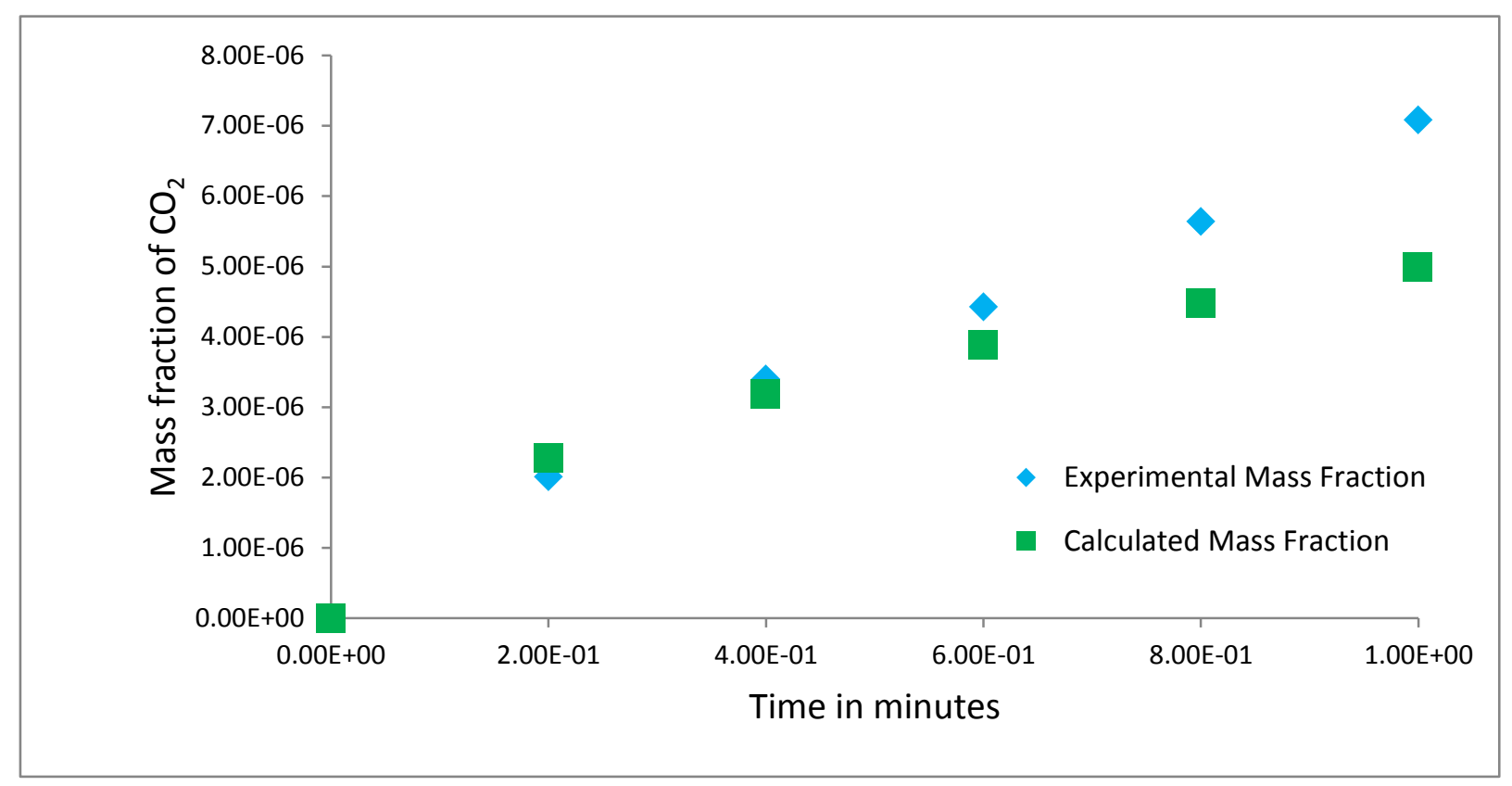

D 7 Experimental versus calculated gas mass of carbon dioxide absorbed in polystyrene with the $+3 \%$ increase in the initial diffusivity estimate

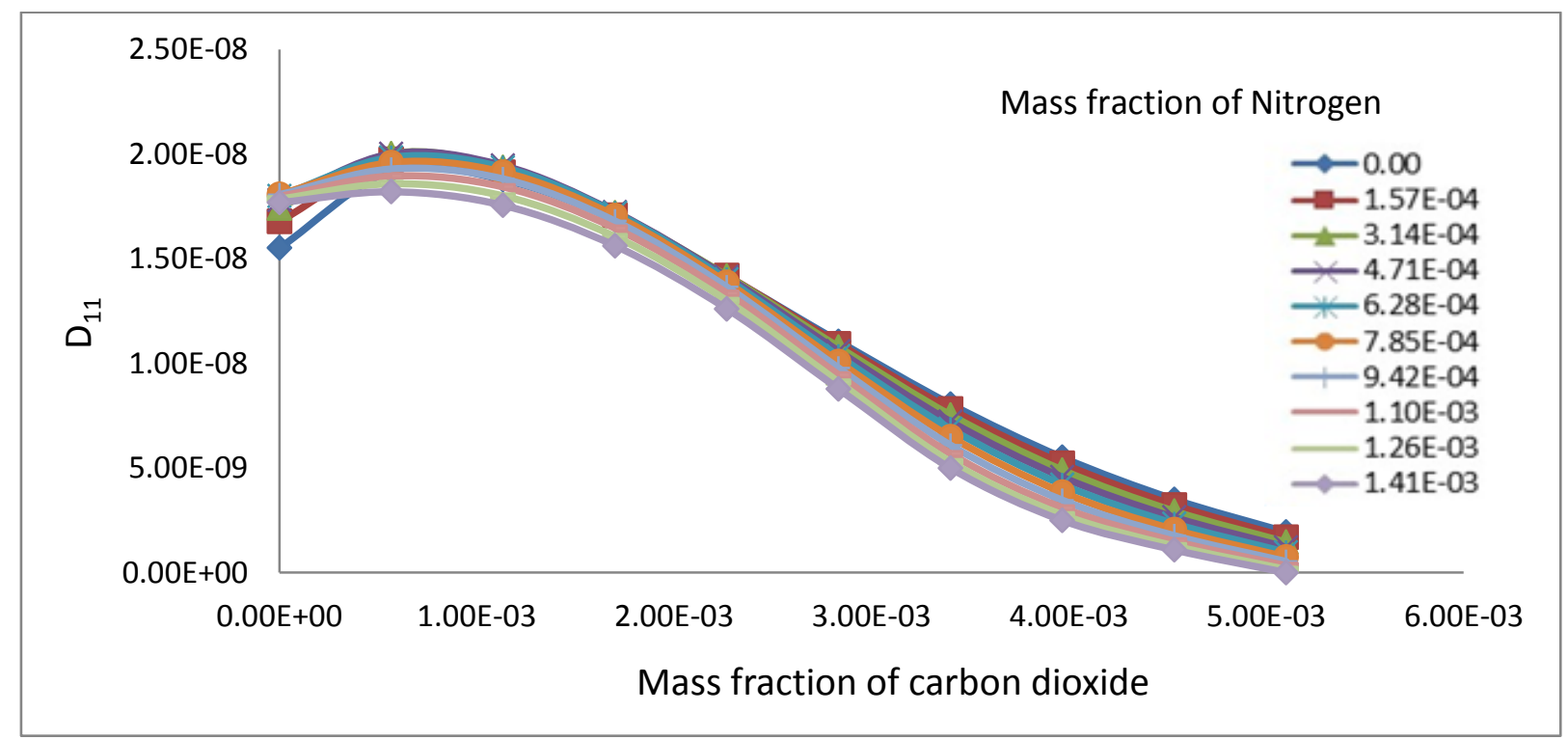

D 8 Main diffusion coefficient D11 with -3\% decrease in initial diffusivity estimate 


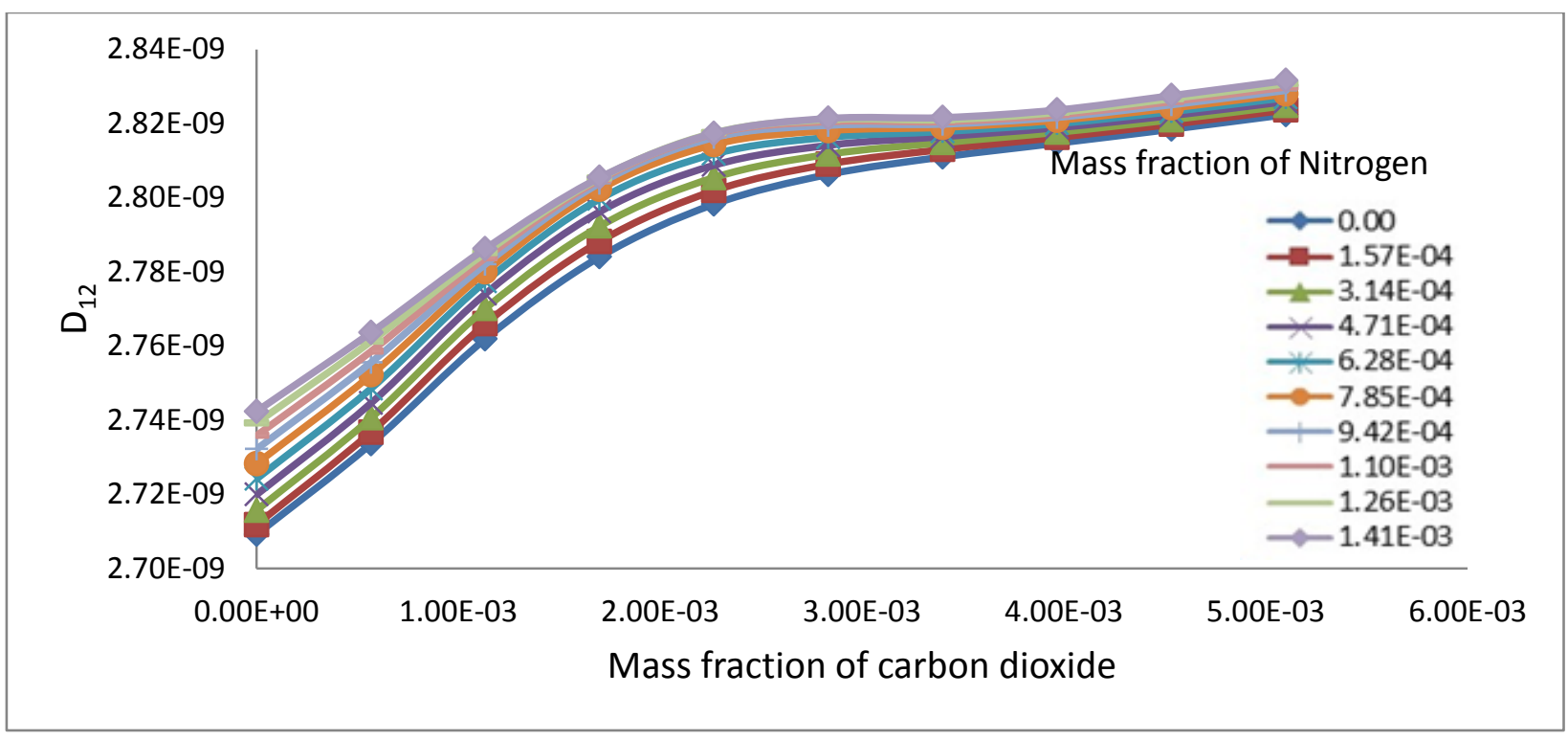

D 9 Cross diffusion coefficient D12 with -3\% decrease in initial diffusivity estimate

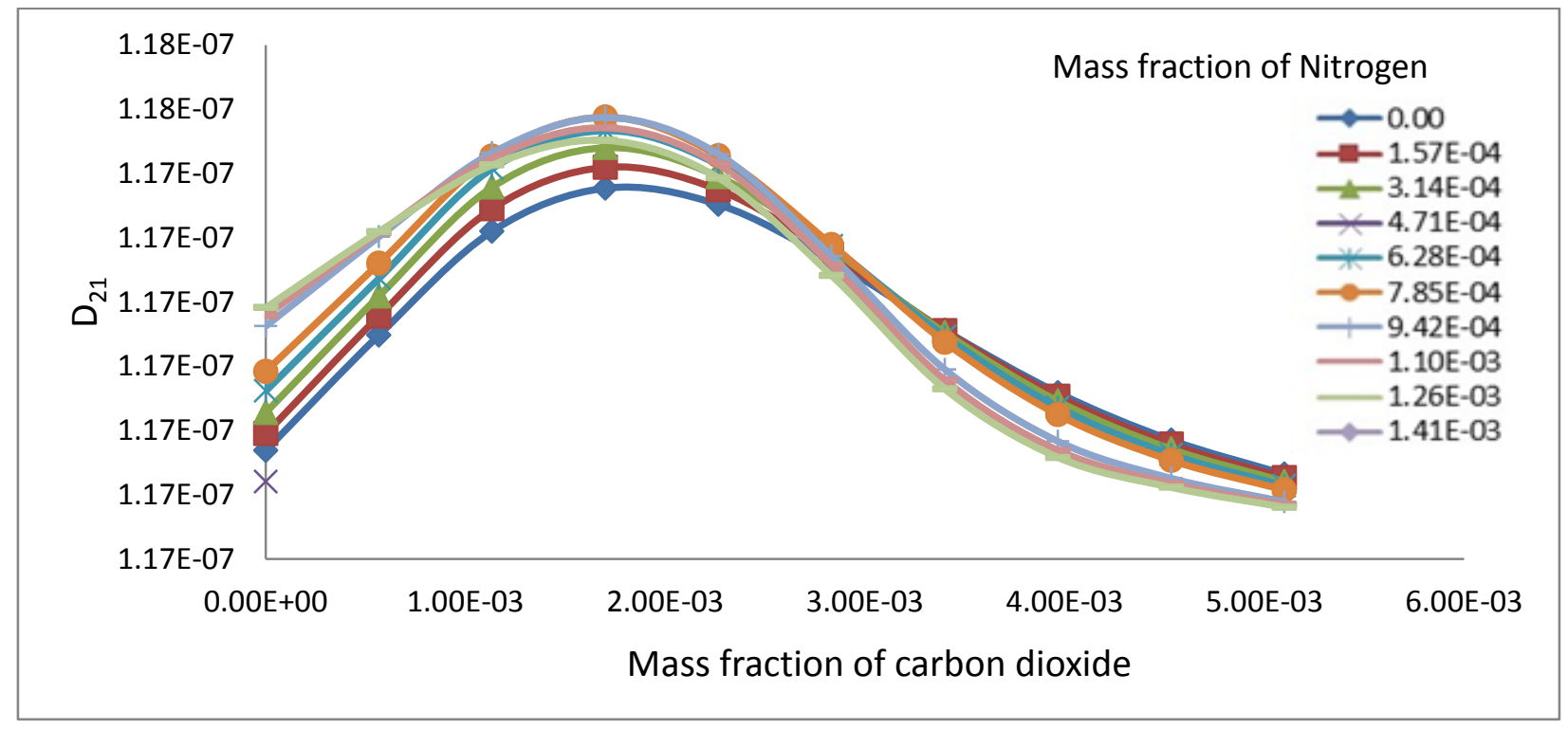

D 10 Cross diffusion coefficient D21 with -3\% decrease in initial diffusivity estimate 


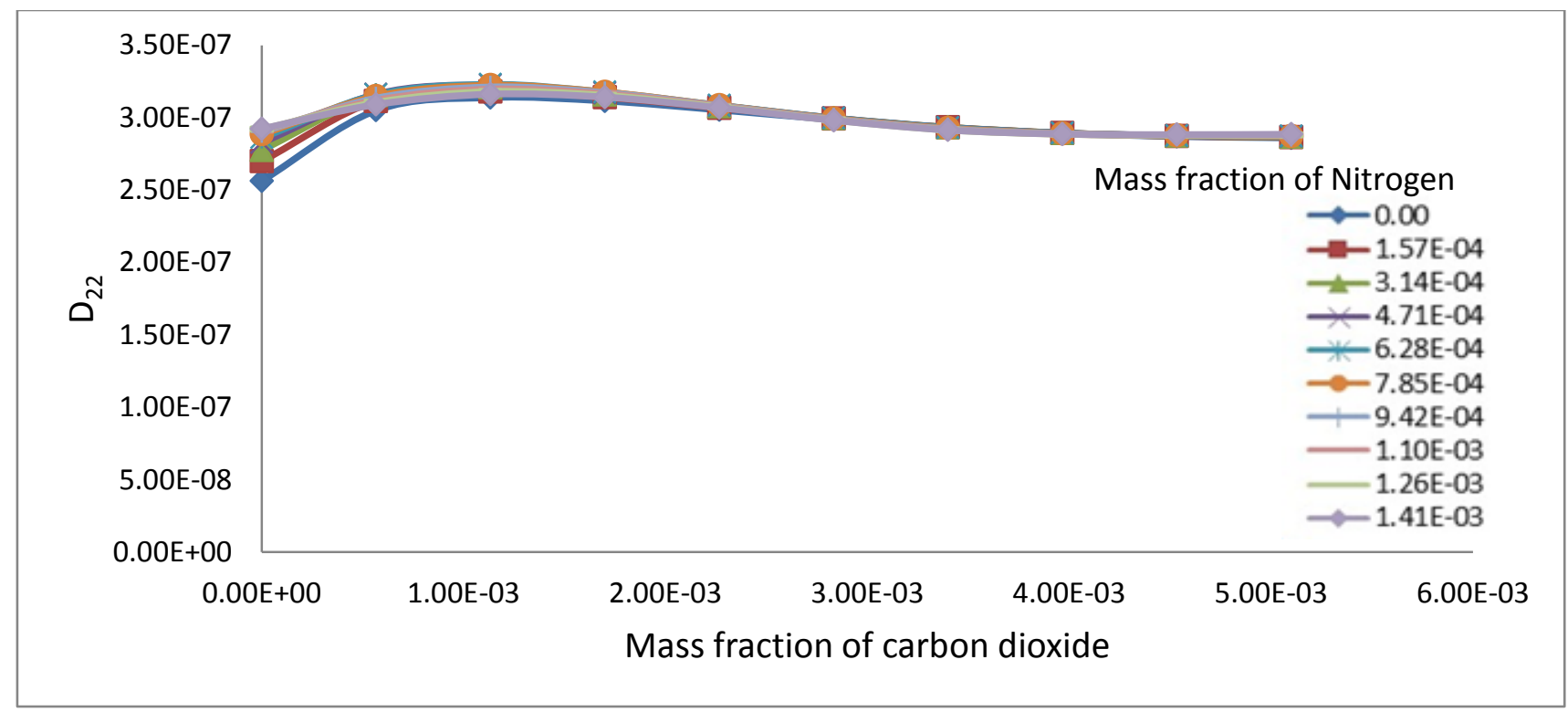

D 11 Main diffusion coefficient D22 with -3\% decrease in initial diffusivity estimate

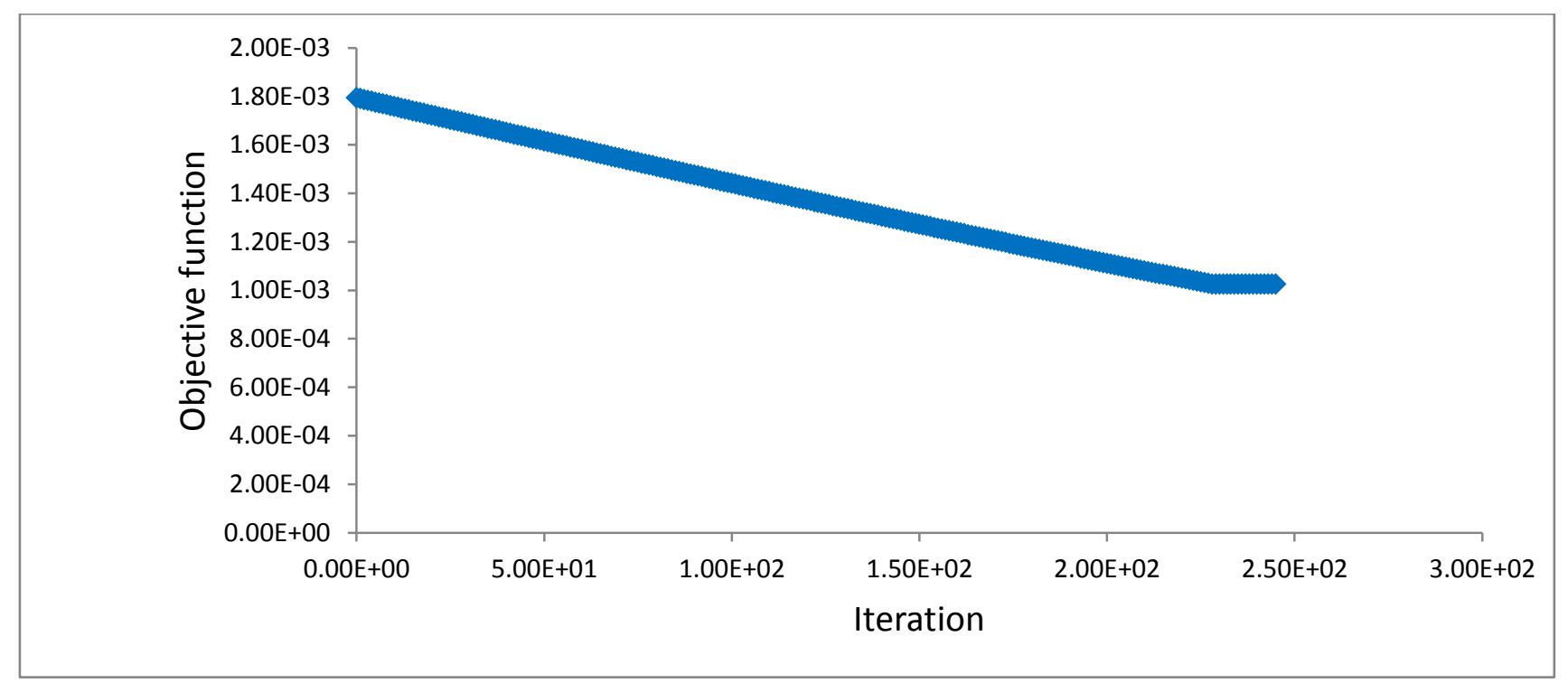

D 12 The convergence of objective functional to the optimum with the $-3 \%$ decrease in the initial diffusivity estimate 


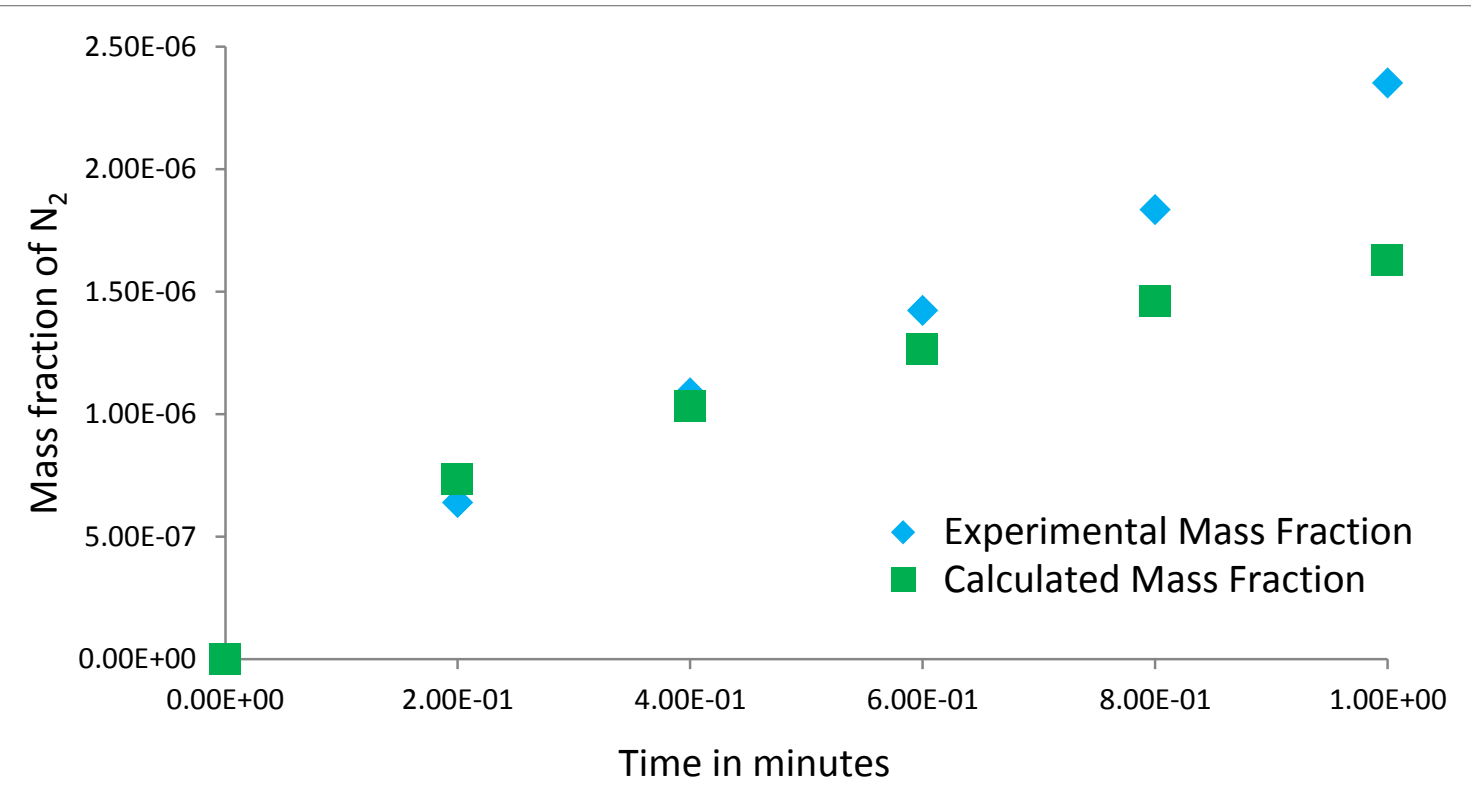

D 13 Experimental versus calculated gas mass of nitrogen absorbed in polystyrene with the $-3 \%$ decrease in the initial diffusivity estimate

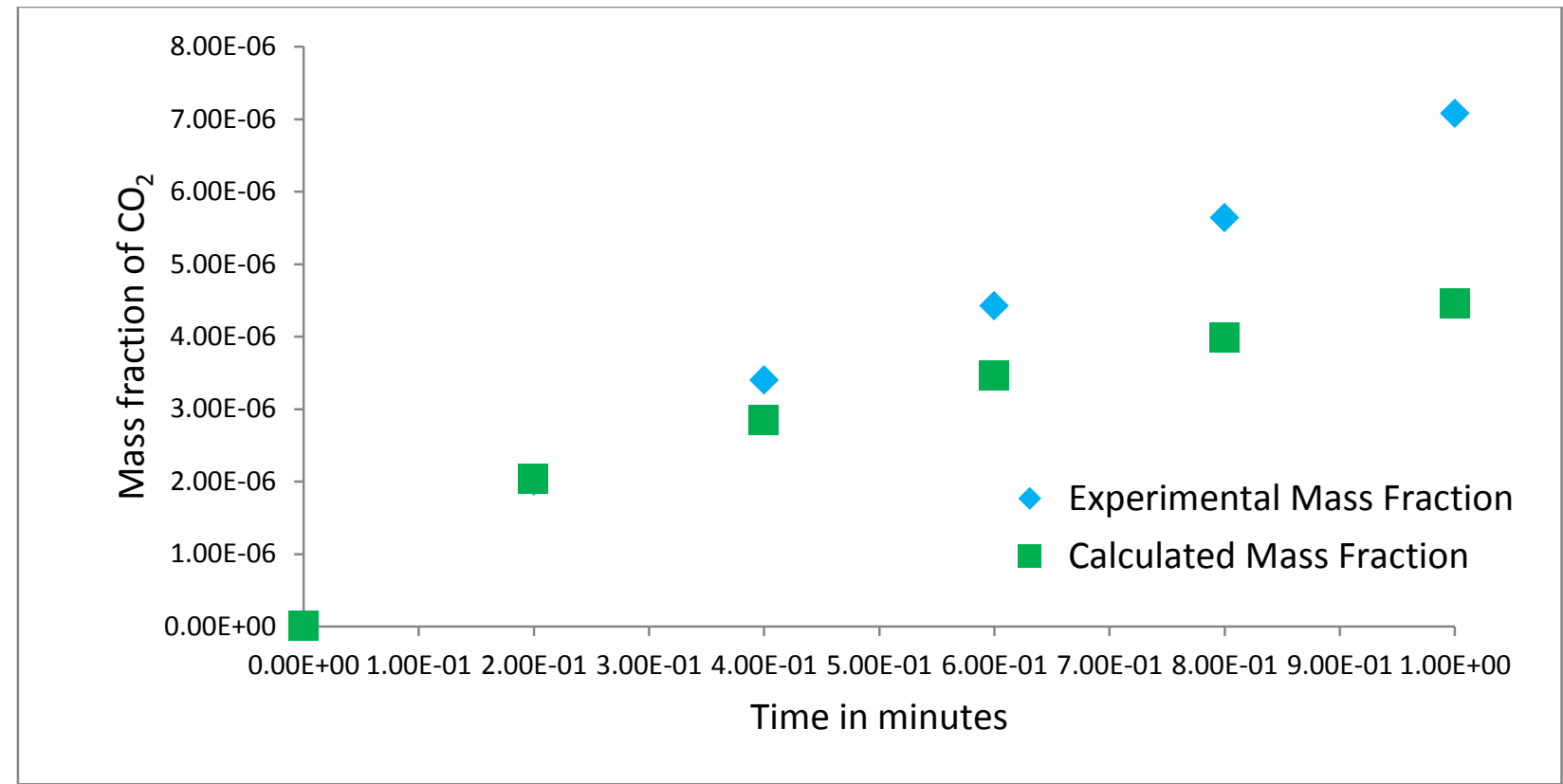

D 14 Experimental versus calculated gas mass of carbon dioxide absorbed in polystyrene with the $-3 \%$ decrease in the initial diffusivity estimate 
